\title{
Real-time Operational Response Methodology for Reducing Failure Impacts in Water Distribution Systems
}

Submitted by Herman Abdulqadir Mahmoud to the University of Exeter as a thesis for the degree of Doctor of Philosophy in Engineering

In March 2018

This thesis is available for Library use on the understanding that it is copyright material and that no quotation from the thesis may be published without proper acknowledgement.

I certify that all material in this thesis which is not my own work has been identified and that no material has previously been submitted and approved for the award of a degree by this or any other University.

Signature: 


\section{ABSTRACT}

Interruption to water services and low water pressure conditions are commonly observed problems in water distribution systems (WDSs). Of particular concern are the unplanned events, such as pipe bursts. The current regulation in the UK requires water utilities to provide reliable water service to consumers resulting in as little as possible interruptions and of as short possible durations. All this pushes water utilities toward developing and using smarter responses to these events, based on advanced tools and solutions. All with the aim to change network management style from reactive to a proactive, and reduce water losses, optimize energy use and provide better services for consumers.

This thesis presents a novel methodology for efficient and effective operational, short time response to an unplanned failure event (such as pipe burst) in a WDS. The proposed automated, near real-time operational response methodology consists of isolating the failure event followed by the recovery of the affected system area by restoring the flows and pressures to normal conditions.

The isolation is typically achieved by manipulating the relevant on/off valves that are located closely to the event location. The recovery involves selecting an optimal combination of suitable operational network interventions. These are selected from a number of possible options with the aim to reduce the negative impact of the failure over a pre-specified time horizon. The intervention options considered here include isolation valve manipulations, changing the pressure reducing valve's (PRV) outlet pressure and installation and use of temporary overland bypasses from a nearby hydrant(s) in an adjacent, unaffected part of the network. The optimal mix of interventions is identified by using a multiobjective optimization approach driven by the minimization of the negative impact on the consumers and the minimization of the corresponding number of operational interventions (which acts as a surrogate for operational costs). The negative impact of a failure event was quantified here as a volume of water undelivered to consumers and was estimated by using a newly developed pressure-driven model (PDM) based hydraulic solver.

The PDM based hydraulic solver was validated on a number of benchmark and real-life networks under different flow conditions. The results obtained clearly demonstrate its advantages when compared to a number of existing methods. 
The key advantages include the simplicity of its implementation and the ability to predict network pressures and flows in a consistently accurate, numerically stable and computationally efficient manner under both pressure-deficient and normalflow conditions and in both steady-state and extended period simulations.

The new real-time operational response methodology was applied to a real world water distribution network of D-Town. The results obtained demonstrate the effectiveness of the proposed methodology in identifying the Pareto optimal network type intervention strategies that could be ultimately presented to the control room operator for making a suitable decision in near real-time. 


\section{PAPERS PPRESENTED BY THE CANDIDATE}

P1 Mahmoud, H. A, Z. Kapelan, and D. Savić (2017), A Response Methodology for Reducing Impacts of Failure Events in Water Distribution Networks, Procedia Engineering, vol.186, p. 218-227, doi:10. 1016/ j.proeng. 2017.03.231.

P2 Mahmoud, H. A., D. Savić, and Z. Kapelan (2017), New Pressure-Driven Approach for Modeling Water Distribution Networks, Journal of Water Resources Planning and Management,143(8), 04017031, doi: 10.1061/(ASCE)WR.1943-5452.0000781.

P3 Mahmoud, H. A, Z. Kapelan, and D. Savić (2017), Segments Identification in Water Distribution Systems by Using Network Topological Matrices, Proceeding to the $15^{\text {th }}$ International Computing \& Control for the Water Industry Conference (CCWI 2017), Sheffield, United Kingdom, doi:10.15131/shef.data.5364145.v1.

P4 Mahmoud, H. A, Z. Kapelan, and D. Savić (2018), Real-time operational response methodology for reducing failure impacts in water distribution systems, Journal of Water Resources Planning and Management, Accepted, doi:10.1061/(ASCE)WR.1943-5452.0000956. 


\section{TABLE OF CONTENTS}

ABSTRACT

PAPERS PPRESENTED BY THE CANDIDATE ......................................... 4

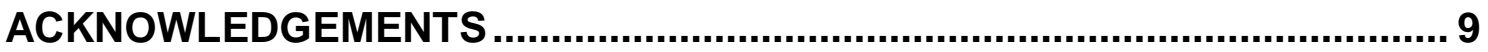

LIST OF FIGURES

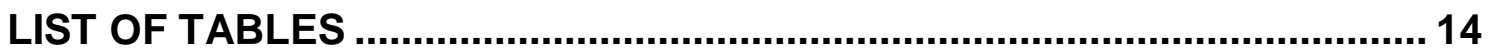

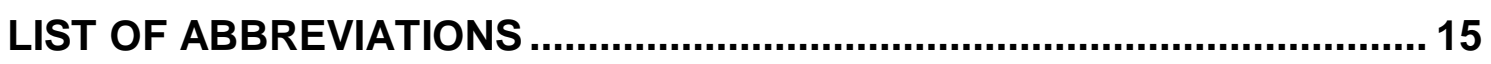

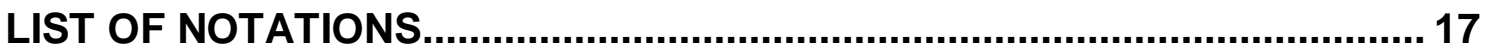

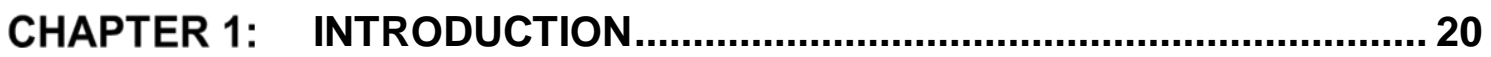

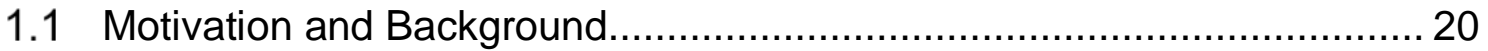

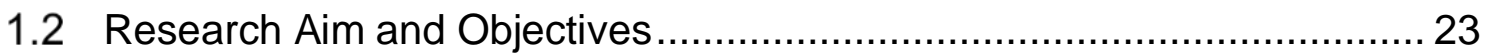

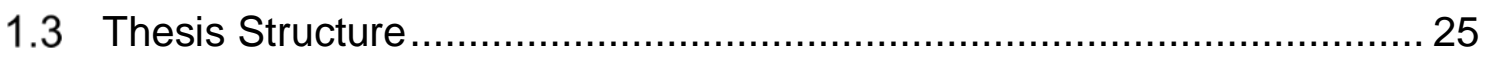

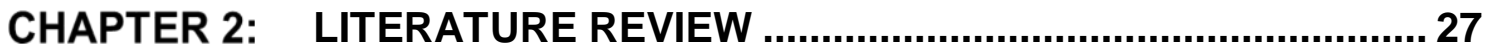

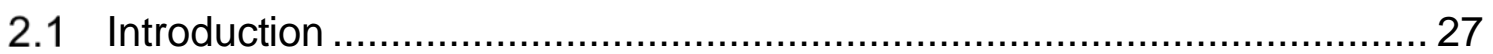

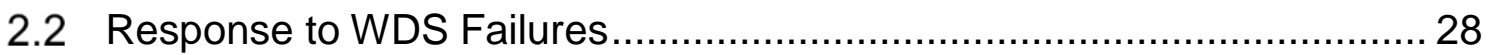

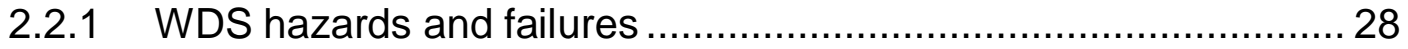

2.2.2 Impacts of failures on the WDS …………............................. 30

2.2.3 WDS failure event management ................................................ 31

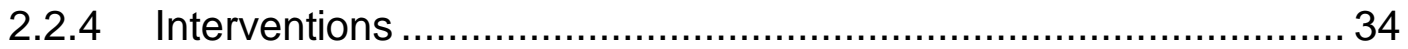

2.2.5 Intervention selection approaches ............................................ 36

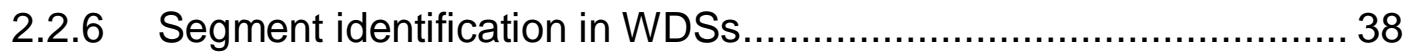

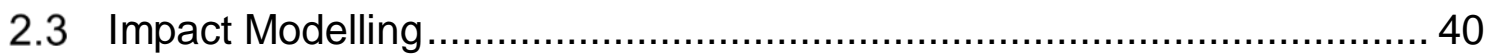

2.3.1 Operational performance assessment ..................................... 40

2.3.2 Modelling of failure events in a WDS ........................................ 43

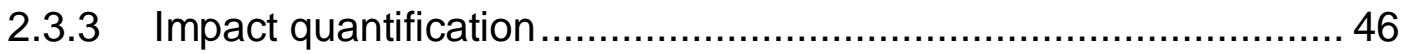


2.3.4 Approaches to model pressure-deficient conditions using DDM

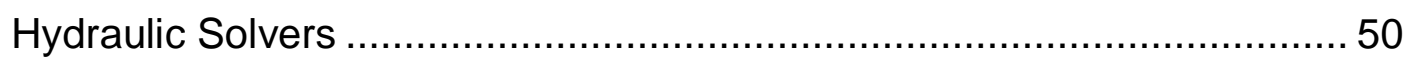

2.3.5 Real-time WDS models........................................................... 53

2.4 Real-time Operational Optimization Models of WDS …........................... 55

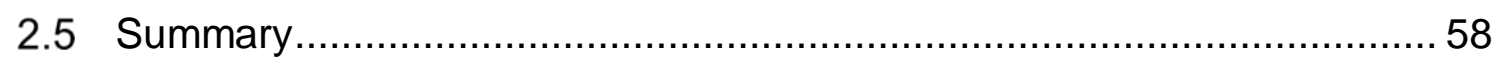

CHAPTER 3: METHODOLOGY FOR REAL-TIME OPERATIONAL RESPONSE TO FAILURE EVENTS IN A WDS ......................................... 59

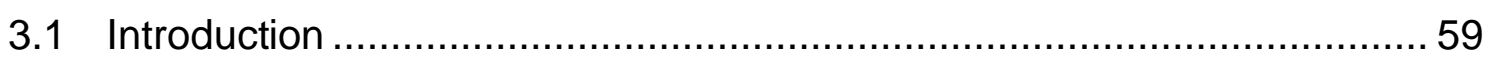

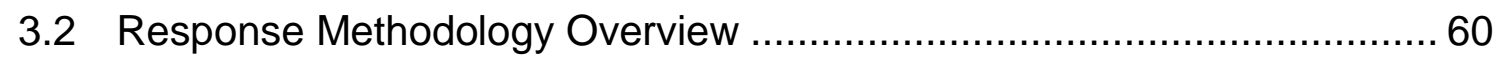

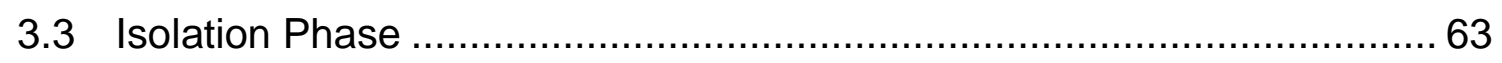

3.3.1 Segment identification methodology .......................................... 66

3.4 Recovery Phase

3.4.1 Recovery interventions ....................................................... 74

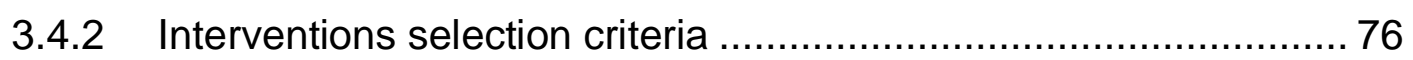

3.4.3 Intervention optimization problem ........................................... 77

3.4.4 Intervention selection methodology .......................................... 79

3.4.5 Simulation of interventions in a hydraulic solver ......................... 85

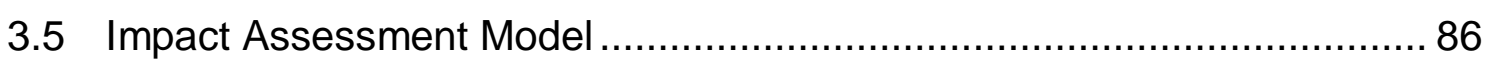

3.5.1 Pressure driven model ......................................................... 87

3.5.2 Impact assessment ............................................................ 91

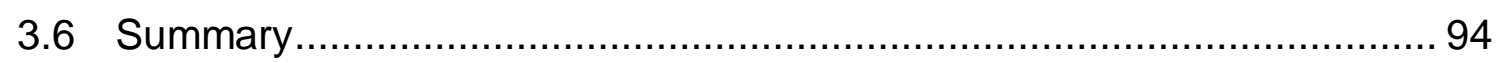

CHAPTER 4: CASE STUDIES ON PRESSURE-DRIVEN MODEL ............... 95

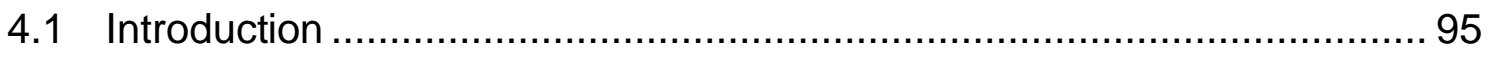

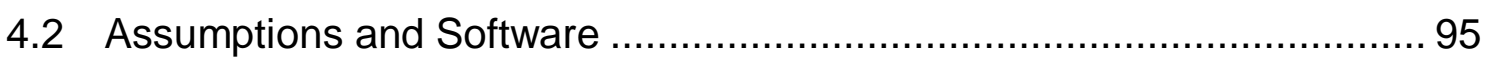

4.3 Case Study 1: Steady-State Simulation in a Simple Looped Network ..... 96

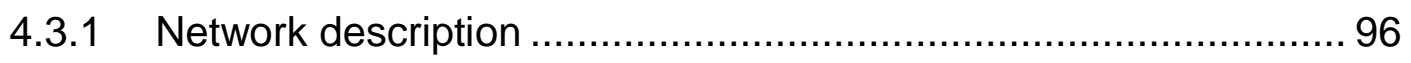




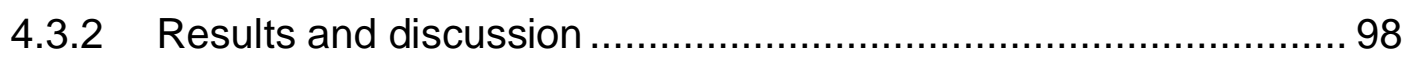

4.4 Case Study 2: Steady-State Simulation in a Large Pipe Networks ........ 104

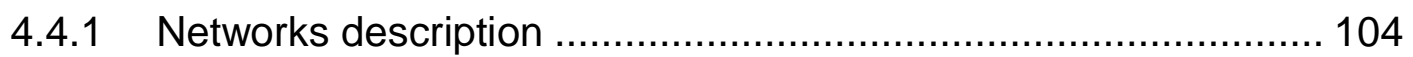

4.4.2 Results and discussion ................................................... 107

4.5 Case Study 3: Extended Period Simulation in a Small Network............. 112

4.5.1 Network description .......................................................... 113

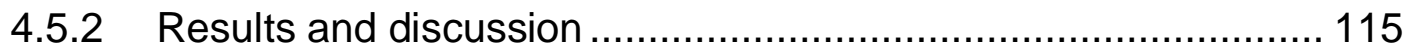

4.6 Case Study 4: Extended Period Simulation in a Large Real-life Network 119

4.6.1 Network description ......................................................... 120

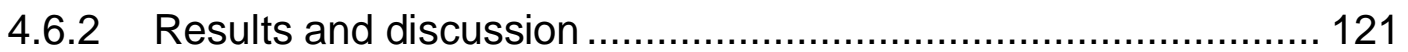

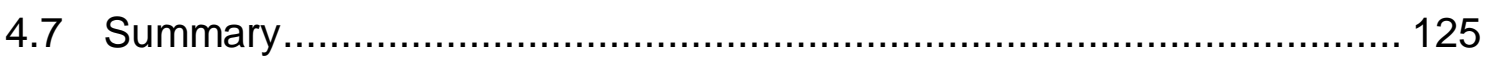

CHAPTER 5: CASE STUDY ON REAL-TIME OPERATIONAL RESPONSE METHDOLOGY 126

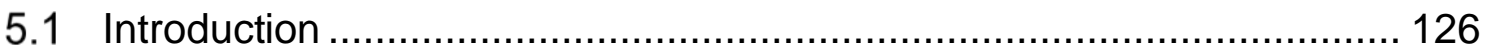

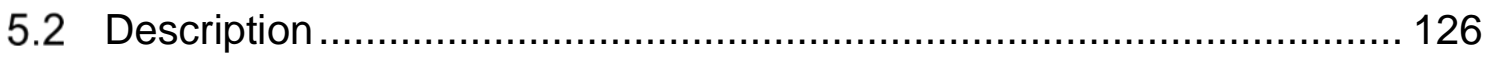

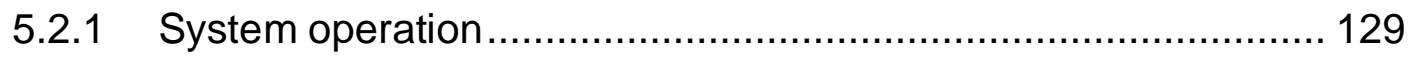

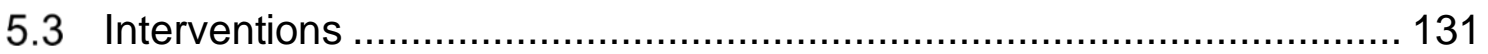

5.3.1 Isolation valves .................................................................. 131

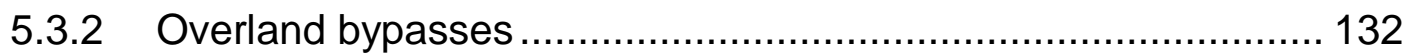

5.3.3 Pressure reducing valves (PRVs) …………............................ 132

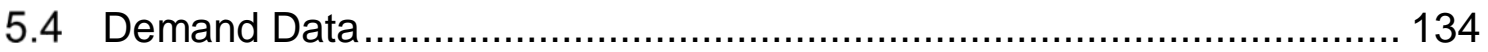

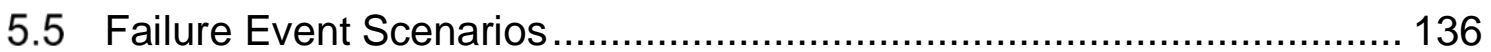

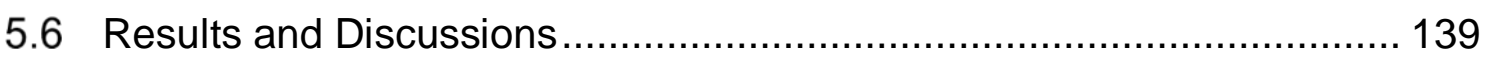

5.6.1 Segment identification analysis............................................ 139

5.6.2 Response in the first failure scenario ..................................... 140 
CHAPTER 6: SUMMARY, CONCLUSIONS AND FUTURE WORK

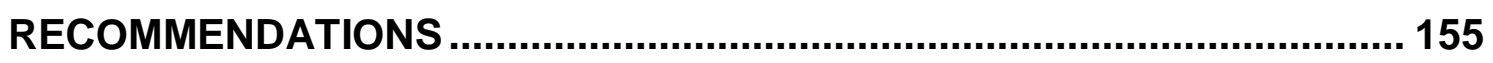

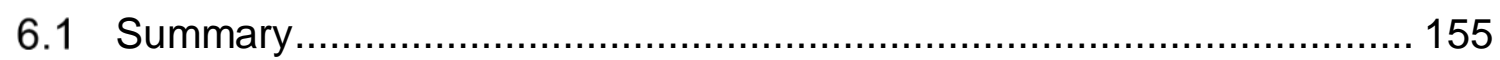

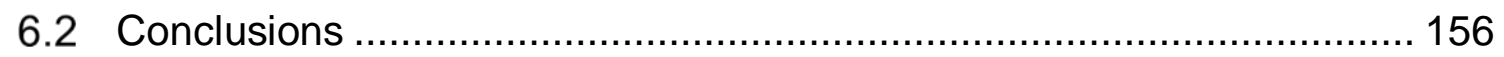

6.2.1 Real-time operational response methodology.......................... 156

6.2.2 Pressure driven analysis..................................................... 158

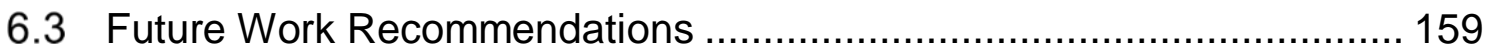

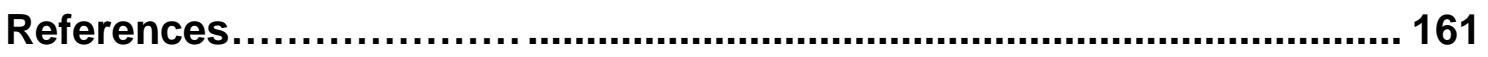

Appendix A Segment Analysis Detail .................................................... 181 


\section{ACKNOWLEDGEMENTS}

Firstly, I would like to express my faithful thanks to my supervisors Professors Zoran Kapelan and Dragan Savic, whose continued support, and guidance throughout this PhD project has been invaluable to the completion of this thesis. I am highly grateful for their tireless assistance, friendly advice, constant feedback, and for sharing their knowledge and expertise, allowing this research to reach its full potential. Honestly, their wide knowledge range in the water system engineering field, highly efficient working style and personal characteristics are something impressed me.

I would also like to acknowledge the financial support from the Kurdistan Regional Government in Iraq, Ministry of High Education and Scientific Research, through Human Capacity Development Program (HCDP). Even with the economic crisis that faced to the Kurdistan, war with ISIS and conflict with the central government in Baghdad, they have tried to continue spend payments to me which contributed towards the successful completion of this thesis.

Also, I gratefully acknowledge the partial financial support from the College of Engineering, Mathematics and Physical Sciences, University of Exeter through hardship program, and I highly appreciate their understanding for finding practical solutions regarding my finance difficulties with my sponsor.

I would also like to thank colleagues and friends in the Centre for Water Systems at the University of Exeter, for creating a friendly working environment and offering support when a much needed mental break was required.

Finally, I would like to thank my family and friends for supporting and understanding me throughout this journey, practically, financially and with moral support, especially my parents. I always knew that you believed in me and wanted the best for me. This thesis is dedicated to all of them and to the soul of my brother Aram (2001-2017). 


\section{LIST OF FIGURES}

Figure 1.1. The outlines of thesis chapters and their interactions................. 25

Figure 2.1. Comparison of different head-flow relationships used in PDM. .... 49

Figure 3.1. Phases of the response methodology. ............................... 61

Figure 3.2. Failure event development stages vs system performance...........62

Figure 3.3. Regular and unintended network segments under $p 2$ failure for $a$ simple network. 66

Figure 3.4. Stages for identifying optimum interventions in the recovery phase.

Figure 3.5. Flow path line definition to the deficient nodal demands. 82

Figure 3.6. The process of estimating decision variables in the proposed response methodology.

Figure 3.7. Representation of isolation valve and hydrant in hydraulic simulation solver. 85

Figure 3.8. Setup at each deficient demand node. 88

Figure 3.9. Flowchart of the SIPDM approach. 90

Figure 4.1. Network layout for case study 1 . 97

Figure 4.2. Results of DDM analysis on the network shown in Figure 4.1......99

Figure 4.3. Results of SIPDM analysis on the network shown in Figure 4.1.. 101

Figure 4.4. Errors observed in (a) total outflow (b) pressure head at demand nodes for networks NW3 and NW4. 107

Figure 4.5. The pressure values observed for nodes in network NW2 using (a) EPANET2.0 (b) SIPDM approach (c) Gorev et al. (2013) method (d) Babu and Mohan (2012) method.

Figure 4.6. Test case study 3 network configuration. 113

Figure 4.7. Demand multiplier factors for case study 3 network. 114 
Figure 4.8. Pressure predictions at demand nodes for case study 3 network using EPANET2.0 solver under increased demands condition. 115

Figure 4.9. Demand satisfaction ratios at demand nodes for case study 3 network using SIPDM approach under increased demands condition. 116

Figure 4.10. Pressure predictions at demand nodes for case study 3 network using EPANET2.0 solver under isolating pipe P7 condition. 118

Figure 4.11. Supply shortfall in case study 3 network under isolating pipe P7 condition. 118

Figure 4.12. Supply shortfall and available pressure head at node N7 in case study 3 network under isolating pipe P7 condition. 119

Figure 4.13. Network layout for case study 4 network. 120

Figure 4.14. Average computational time comparison for EPS for the normal flow scenario for case 4 network 121

Figure 4.15. Errors observed in term of the total system flow between SIPDM and EPANET2.0 for the normal flow scenario for case 4 network. 122

Figure 4.16. Number of nodal demands deficient under abnormal flow scenario in the case 4 network 123

Figure 4.17. Water depth at tank T4 for case 4 network. 124

Figure 4.18. The prediction of (a) pressure heads and (b) flows at node J278 for case 4 network. 124

Figure 5.1. D-Town network layout (Marchi et al. 2012). 127

Figure 5.2. Schematic representation of the Guidolin et al. (2012) solution: modifications to the original network (Marchi et al. 2012) are shown in black. 128 Figure 5.3. D-Town system schematic (after Ostfled et al. 2012) 129

Figure 5.4. Water depth observed at the D-Town network tanks under normal system operation. 130

Figure 5.5. Tank description in EPANET2.0. 131

Figure 5.6. The location of pipe bursts, isolation valves, hydrant points, and PRVs in D-Town network. 
Figure 5.7. Demand multiplier factors for D-Town network.

Figure 5.8. Allocated (a) base demand (b) number of consumers at each node for D-Town network.

Figure 5.9. Outflow profile from the pressure sensitive burst at (a) pipe P307 (b) pipe P424.

Figure 5.10. Segmentation details for failure at pipes: (a) P307 and (b) P424.

Figure 5.11. The estimated impact of pipe P307 failure on the system (including the isolated area) in terms of (a) shortfall (b) total cumulative volume of water undelivered. 141

Figure 5.12. Water depth at tank $\mathrm{T} 2$ and $\mathrm{T} 4$ under (a) pipe $\mathrm{P} 307$ failure scenario (b) normal operation scenario. 142

Figure 5.13. The estimated impact of isolating pipe $P 307$ on the isolated nodes in terms of (a) shortfall (b) total cumulative volume of water undelivered....... 143

Figure 5.14. The network showing isolated area and the direction of flows for pipe P307 failure scenario at most critical time of 20:00 144

Figure 5.15. Best ranked operational recovery interventions for pipe P307 failure scenario. 145

Figure 5.16. The location of recovery interventions for INV=3 (OV5, OV217, and OV225) for P307 failure scenario. 146

Figure 5.17. The final optimal recovery interventions for pipe P307 failure. .. 147

Figure 5.18. The estimated impact of pipe P307 failure on the system (including the isolated area), after introducing recovery interventions, in terms of (a) shortfall (b) total cumulative volume of water undelivered. 147

Figure 5.19. The estimated impact of isolating pipe P424 on the DMA5 nodes in terms of (a) shortfall (b) total cumulative volume of water undelivered. 149

Figure 5.20. Water depth at tank T6 and T7 under pipe P424 failure scenario. 150

Figure 5.21. The final optimal recovery interventions for pipe P424 failure scenario. 151 
Figure 5.22. The estimated impact of pipe P424 failure on the system (including the isolated area), after introducing recovery interventions, in terms of (a) shortfall (b) total cumulative volume of water undelivered. 152

Figure 5.23. Effect of the number of interventions on system recovery at time 15:00 for: (a) INV=0, (b) INV=2, (c) INV=3, (d) INV =4. 153 


\section{LIST OF TABLES}

Table 1.1. Interaction between specific objectives, research questions, thesis

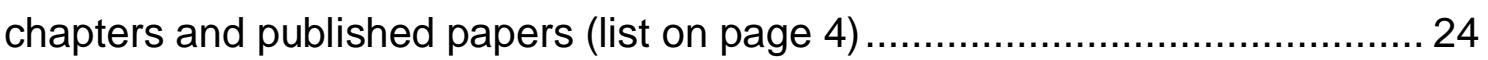

Table 2.1 Potential hazards and their classification in WDSs. 29

Table 3.1. Node-Link and Valve-Location matrices for the network in Figure 3.3.

Table 3.2. Deficiency $\left(A_{v d}\right)$ and $V_{p}$ matrices for the network in Figure 3.3.......69

Table 3.3. Matrix $V$ and $V^{m}$ (with $m=4$ ) for the network in the Figure 3.3. ....... 70

Table 3.4. Segments isolation detail for the network in the Figure 3.3........... 71

Table 3.5. Node-Link and $V^{m}$ matrices for the network in Figure 3.3 after removing

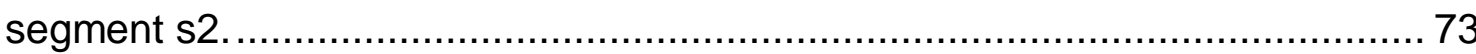

Table 3.6. Properties of added elements to deficient demand nodes. 88

Table 4.1. Properties for (a) nodes (b) pipes for the network shown in Figure 4.1.

Table 4.2. Comparison of the results obtained for case study 1 network under normal flow condition for the source head $60 \mathrm{~m}$. 102

Table 4.3. Characteristics of network models used in the performance tests for case study 2 . 106

Table 4.4. Comparison of the results observed in networks NW1 and NW2 using EPANET2.0 solver and SIPDM approach. 108

Table 4.5. Results comparison for (a) DSR (b) computational time performance in Steady-State simulation in a large pipe networks for case study 2 ............ 110

Table 4.6. Properties for (a) nodes (b) pipes for case 3 network. 114

Table 5.1 Modifications to the original D-Town (Marchi et al. 2012) by Guilodin et al. (2012) 128

Table 5.2. Properties for the PRVs in the D-Town network. 133

Table A1. Segment analysis for D-Town 181 


\section{LIST OF ABBREVIATIONS}

ADF Available demand fraction

ANNs $\quad$ Artificial Neural Networks

CPU Central Processing Unit

CV Check Valve

DDM Demand-driven model

DMA District metred area

DN Dummy node

DSR Demand satisfaction ratio

DSS Decision Support System

D-W Darcy-Weisbach

EM Emitter

EPS Extended period simulation

FCV Flow Control Valve

FDD Fraction of delivered demand

FDV Fraction of delivered volume

GAs Genetic Algorithms

GGA Global Gradient Algorithm

GIS Geographic Information System

GUI Graphical User Interface

HD-DDS Hybrid discrete dynamically dimensioned search

H-W Hazen-Williams

LPS Scale of the Low Pressure

MOEAs Multi-objective evolutionary algorithms

NHFR Nodal head-flow relationship 
NSGA-II Non-dominated Sorting Genetic Algorithm-II

OFWAT UK Office of Water Services

PDM Pressure-driven model

PRV Pressure reducing valve

PSPF Demand supplied at adequate pressure

SCADA Supervisory Control and Data Acquisition

SID Supply Interruption Duration

SIM Service Incentive Mechanism

SIPDM Single Iteration Pressure-Driven Model

SIS Supply interruption Scale

SPI System performance indicator

USEPA United States Environmental Protection Agency

WDS Water distribution system 


\section{LIST OF NOTATIONS}

$A_{n l}^{s i} \quad$ node-link matrix for segment si

$x_{i}^{I V} \quad$ the status of isolation valve $i$

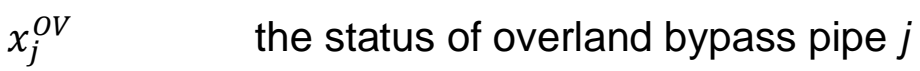

$x_{k}^{P R V} \quad$ the pressure setting at the exit of PRV $k$

$\Delta t \quad$ simulated hydraulic time step, $\mathrm{s}$

$A_{n l} \quad$ node-link matrix

$A_{v d} \quad$ valve deficiency matrix

$A_{v l} \quad$ valve-location matrix

$C_{d} \quad$ emitter coefficient

CINV total operational cost for the recovery interventions

$E L_{n} \quad$ elevation of node $\mathrm{n}, \mathrm{m}$

$f(X) \quad$ objective function

IM impact of failure over the time horizon considered, $\mathrm{m}^{3}$

IMR impact of doing nothing over horizon $T_{h}, \mathrm{~m}^{3}$

in(n) the set of pipes that are supplying flow from node $n$ at time $t$

INV $\quad$ the maximum allowed number of interventions

IV candidate isolation valve whose operation mode may change during recovery intervention phase over horizon $T_{h}$

$K_{l} \quad$ the head friction loss coefficient at link I

$L \quad$ the number of network links

LD distance between two hydrant points, $\mathrm{m}$

$L D_{\max } \quad$ maximum allow distance between two hydrant points, $\mathrm{m}$

$N \quad$ number of network nodes 
niv

$n$

$n_{j}$

$n_{n}$

nob

$n p r$

$n_{s}$

$n_{v}$

out(n)

OV

$P_{d}(t)$

$P_{n, a v l}$

$P_{n, \min }$

$P_{n, \text { req }}$

PRV

$P_{u}(t)$

$Q_{l}(t)$

$Q_{n, d e l}(t)$

$Q_{n, \text { req }}(t)$

$t$

$t_{c}$

$t_{d}$ total number of feasible isolation valves in the network for a specific failure event in a specific WDS

total number of pipes and pumps in the network

total number of junctions in the network

empirical exponent coefficient at node $n$

total number of feasible overland bypass pipes in the network for a specific failure event in a specific WDS

total number of feasible PRVs in the network for a specific failure event in a specific WDS

total number of source nodes in the network

total number of isolation valves in the network

the set of pipes that are delivering flow from node $n$ at time $t$

candidate overland bypass pipe whose operation mode may change during recovery intervention phase over horizon $T_{h}$

the head pressures at the downstream ends of pipe / at time $t, \mathrm{~m}$

available pressure head at node $n, \mathrm{~m}$

minimum pressure head at node $n, \mathrm{~m}$

required pressure head to deliver required demand at node $n, \mathrm{~m}$

candidate PRV whose operation mode may change during recovery intervention phase over horizon $T_{h}$

the head pressures at the upstream ends of pipe / at time $t, \mathrm{~m}$

flow rate in link $I=(u, d)$ at time $t, I / s$

delivered demand at node $n$ and time $t, 1 / \mathrm{s}$

required demand at node $n$ and time $t, \mathrm{I} / \mathrm{s}$

simulation time, hours

time when set of optimal operational interventions identified

moment of detection of the failure event 


\begin{tabular}{|c|c|}
\hline$t_{e}$ & end time of impact horizon, hours \\
\hline$t_{f}$ & moment of locating of the failure event \\
\hline$T h$ & horizon time, hours \\
\hline$t_{i}$ & moment of isolation of the failure event \\
\hline$t_{0}$ & moment of occurrence of the failure event \\
\hline$t_{r}$ & starting time of recovery phase \\
\hline$V$ & symmetric matrix \\
\hline$w_{0}$ & $\begin{array}{l}\text { unit cost weight for installing an overland bypass pipe in the } \\
\text { network }\end{array}$ \\
\hline$w_{p}$ & unit cost weight for changing the setting of a PRV in the network. \\
\hline$w_{v}$ & $\begin{array}{l}\text { unit cost weight for changing the status of an isolation valve in the } \\
\text { network }\end{array}$ \\
\hline$X$ & decision variables vector for operational interventions \\
\hline$\alpha$ & Hazen-Williams coefficient \\
\hline Y & emitter exponent \\
\hline
\end{tabular}




\section{CHAPTER 1: INTRODUCTION}

\subsection{Motivation and Background}

Increasing demand on water, rising energy price, diminishing water sources, aging water infrastructure, and population growth are serious problems facing water utilities worldwide. In addition to that, the current regulations require water utilities to provide more reliable potable water to consumers than before in terms of quality, less number of service interruptions, and adequate pressure at consumers' taps with minimum carbon footprint level. All of the above concerns push water utilities toward bringing and using smarter tools and solutions in operation and management of a Water Distribution System (WDS) under both normal and abnormal conditions. All with the aim to change network management style from reactive to a proactive, reduce water losses, optimize energy use and provide better services for consumers.

Water distribution system is considered to be one of the essential infrastructures in any city. The main purpose of a WDS is to convey potable water via pipes and other network equipment such as pumps from sources to household, commercial, and industrial consumers, at sufficient pressure and quantity (i.e., flow), while satisfying microbial and chemical standards. WDS tends to achieve these goals and work efficiently most of the time under normal operational conditions (i.e., normal demand stress condition). However, the expansive nature of a WDS makes it vulnerable to failures, faults, and disruptions. For example, pipe bursts, leakage, and equipment failure are common events and occur regularly in WDS operations. These events arise mainly due to the ageing of water infrastructure, power outage, earthquakes, intentional/ unintentional human acts, etc. The performance of a WDS is frequently deteriorated under these events if they not timely detected, located and repaired. This, in turn, produced inconvenient impacts (e.g., pressure drop, supply interruptions, water discolouration) to the consumers, economic losses to utilities and negative effects to the environment (e.g., damages to surrounding property).

In the United Kingdom (UK) great efforts have been invested in reducing and preventing the number of failure events through applying ongoing rehabilitation and maintenance plans, as well as installing more monitoring tools (e.g., smart meters, sensors) to allow water utilities to control these events proactively and in 
an efficient manner. However, due to the stochastic nature of failure events especially unplanned one such as pipe bursts, it is very hard to predict their occurrence as well as completely estimate and eliminate the potentially generated negative impacts. Thus, the need for having a more efficient, economic, self-healing triggered and robust response emergency methodologies to failure events in real-time is significant. This is important to protect water system from adverse impacts, improve water utilities operational performance, and provide uninterrupted services to consumers.

In general, a good response emergency management plan to limit/prevent failure event and its produced negative impacts, especially for sudden one (i.e., pipe burst), comprises three main phases: (1) a real-time detection system, here data (e.g., pressure, flow, quality) are collected from network monitoring equipment such as supervisory control and data acquisition (SCADA), smart meters, and network sensors. These data are then analysed using recent data mining, pattern recognition, mathematical and statistical methods, and network hydraulic and quality solvers to visualize, detect and locate the source of failure event; (2) realtime alarm system; and (3) real-time response and decision support system to recover WDSs from the negative impacts.

This study will specifically focus on the third phase (i.e., response). Water utilities are encouraged to prepare and develop the response, and failure emergency management plan to reduce/prevent negative impacts for the situation when event confirmed (e.g., post-event stage). The response plan typically involves a rapid identification and quantification of system losses and impacts (i.e., impact assessment, notify affected consumers). This followed by providing a number of interventions and alternative temporary corrective action plans for network operators aimed at alleviating (or possibly preventing) the potential negative consequences and to return to pre-event failure operation condition. This may involve, for example, isolating the location of the failure event and affected area, provide alternative water supply options, redistributing network flows or readjustment of flow patterns, manipulating pressure setting within the water system, and using temporary overland bypasses until the required maintenance plan performed. All with the aim to improve flow conditions (e.g., pressure and quality) in the affected area and increase system resilience and reliability under failure conditions. 
Hydraulic and water quality models (e.g., EPANET) combined with mathematical programming methods, such as artificial neural networks, heuristic optimization and genetic algorithms, have been extensively used in WDSs (Prasad and Park 2004; Boulos and Wiley 2013; Fu et al. 2013) for addressing different problems such as for example optimal design for network pipes size, strategical planning and optimal pumping schedule. However, most of these models work offline, and they are unable to perfectly predict the current behaviour and state of a WDS for operational purposes, especially under contingency situations (e.g., pipe bursts).

Recently, the real-time modelling in WDSs is a topic of growing interest aiming at making decisions in a very short time in operation process. These models can help network operators to predict the WDS behaviour under different operational conditions, improve the performance and ensure more reliable operations at minimum cost and in near real-time, rather than relying solely on capital improvements. For example, operators can quickly assess the impacts of a sudden pipe burst as well as generate near-optimal solutions for enhancing system operation. However, few models or systematic algorithms exist to support the response and management process of failure consequences.

Indeed, to date, most models and related studies in the field of response to failure events have been limited to primarily identifying for example the location of the failure event (Laucelli et al. 2016), vulnerability analysis (Laucelli and Giustolisi 2015), strategic reliability (Bouchart and Goulter 1991; Fujiwara and Li 1998; Giustolisi et al. 2008b), and risk analysis (Bicik et al. 2009; Seth et al. 2016). Despite the consistent approaches that water utilities offered towards post-event failure management, limited studies have focused on the elaboration of timely optimum intervention strategies in the field. Possible reasons for the shortage of near real-time post-event failure applications could be in the difficulties imposed by the large amount of applicable data, simulation models, dynamically changing environment, computational speed constraints and spatial analysis.

The work presented in this thesis discusses the development, implementation, and application of a near real-time response methodology for post-event failure, which forms a fundamental part of an overall near real-time Decision Support System (DSS) in WDS operation. The developed methodology can help WDS operators for making more informed support decisions, mitigating adverse and prioritizing timely interventions in more smarter and effective ways. Also, the 
presented methodology can provide WDS operators considerable management benefits involving greater operational efficiency, emergency preparedness, reduced water loss and shortened response time, all with the aim to improve customer service and protect WDSs. Finally, the proposed methodology also can provide indirect benefits to water utilities through avoiding the UK Office of Water Services (OFWAT) penalties related to Service Incentive Mechanism (SIM) and Serviceability Indicator Measures, this in turn, will favourably enhance the water utilities profile and increase the consumers and government trust in the water utilities.

\subsection{Research Aim and Objectives}

The overall aim of this research is to develop and implement a new near realtime optimum response methodology to reduce and prevent negative consequences of an unplanned event in a WDS with focus on pipe bursts, and restore flow conditions in the network to normal service level once the event has been detected and located (i.e., post-event stage). This aim is achieved through the following specific objectives:

1. To conduct in depth review of a wide range of the existing response realtime approaches in operating WDS under failure conditions (i.e., pipe burst). Specifically, the review will focus on the identification and evaluation of the approaches and solution methods that can be used to respond to various failure events in a WDS.

2. To develop a response methodology for reducing the negative consequences of unplanned failure events on consumers in near real-time in a WDS. This will include identification of suitable operational intervention, methodology for impact assessment of these interventions and ultimately methodology for selection of optimal interventions.

3. To develop new methodology for pressure-driven modelling of a WDS fo impact assessment. The above will be approached from an operational, rather than strategic perspective to evaluate the effect of recovery interventions and enable near real-time decision-making.

4. To test, verify and demonstrate the effectiveness and performance of the impact assessment model and related pressure-driven hydraulic model for quantifying negative consequences resulting from unplanned events on a number of benchmark and real-life WDSs. 
5. To test, verify and demonstrate the effectiveness and efficiency of the new response methodology for reducing negative consequences and identifying Pareto optimal recovery interventions in near real-time on a real-life case study.

6. To summarise the work completed, key findings obtained and provide recommendations for future work.

The implementation of the aforementioned objectives aims to answer the project questions summarized in Table 1.1 (references to thesis chapters and related candidate papers are also indicated).

Table 1.1. Interaction between specific objectives, research questions, thesis chapters and published papers (list on page 4)

\begin{tabular}{|c|l|c|c|}
\hline Objective & \multicolumn{1}{|c|}{ Research Question } & $\begin{array}{c}\text { Thesis } \\
\text { Chapter }\end{array}$ & $\begin{array}{c}\text { Related } \\
\text { Papers }\end{array}$ \\
\hline Objective 1 & $\begin{array}{l}\text { What are the major gaps in existing } \\
\text { literature with regard to the operational } \\
\text { response to pipe bursts and other failure } \\
\text { events in a WDS? }\end{array}$ & Chapter 2 & - \\
\hline Objective 2,3 & $\begin{array}{l}\text { What is the best way to respond to a WDS } \\
\text { failure event in near real-time? }\end{array}$ & Chapter 3 & P1, P3, \\
\hline Objective 4 & $\begin{array}{l}\text { Is the impact assessment model based on a } \\
\text { pressure-driven modelling approach valid? }\end{array}$ & Chapter 4 & P2 \\
\hline Objective 5 & $\begin{array}{l}\text { Are the proposed recovery interventions } \\
\text { able to reduce negative consequences of a } \\
\text { pipe burst? }\end{array}$ & Chapter 5 & P3, P4 \\
\hline Objective 6 & $\begin{array}{l}\text { What are the key findings of the research } \\
\text { work conducted and related implications for } \\
\text { engineering practice? }\end{array}$ & Chapter 6 & $\begin{array}{c}\text { All } \\
\text { papers }\end{array}$ \\
\hline
\end{tabular}




\subsection{Thesis Structure}

This thesis is divided into six chapters, and the relationship between them are shown in Figure 1.1. The present chapter (Introduction) provides the background and motivation of the research project, the aim and objectives to be achieved, and the research structure.

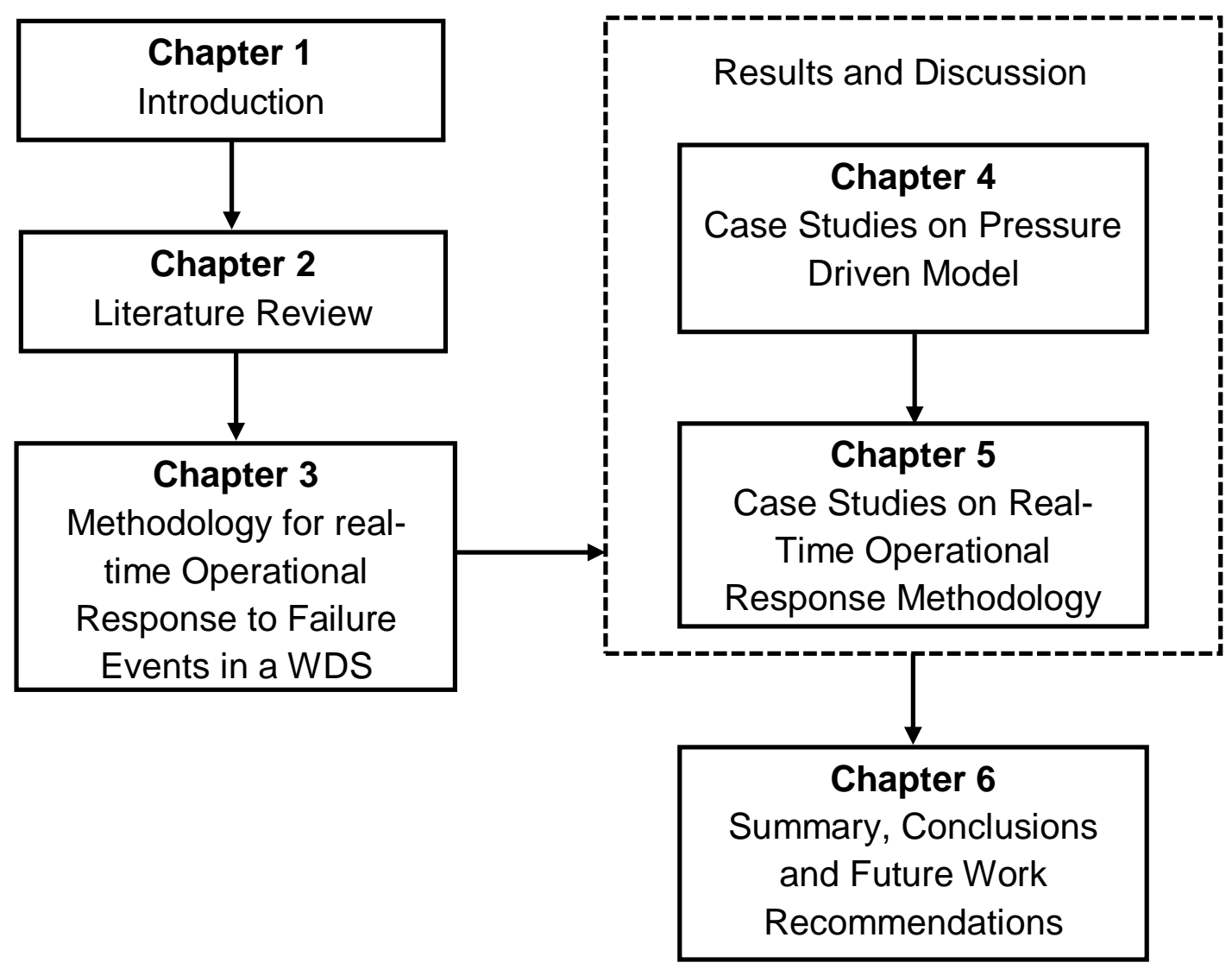

Figure 1.1. The outlines of thesis chapters and their interactions.

Chapter 2 presents an overview of the core topics and most relevant existing literature related to this study, in order to identify research gaps and frame the thesis objectives. The review includes the following main topics: response approaches and techniques to failure events (for both pre- and post-event stages) in WDSs, the approaches for modelling and assessing the impact of failure events and interventions, and methods for optimising the WDS operation/operational interventions including related criteria and optimisation techniques.

In Chapter 3 the overall detail for the new developed near real-time response methodological approach to failure event impacts in WDSs is presented. 
Specifically, an overview of the response phases is given first. Next, a new segmentation approach for performing isolation phase (i.e., first response phase), which is necessary to identify the failure segments (e.g., interrupted nodes and pipes from water sources) and valves to isolate event location, is presented. This is then followed by describing recovery phase, this involves selection criteria (e.g., cost-benefit objectives) and solution method (i.e., optimization) used to identify optimum recovery interventions in near real-time. Once this done, an operational impact assessment model, utilizing the new pressure-driven modelling to quantify failure impacts, is described.

In Chapter 4 the performance and efficiency of the developed impact assessment model that developed in Chapter 3 is tested, verified and illustrated under different flow conditions on a number of benchmarks and real-life case WDSs found in the literature. The case studies considered are divided into four groups based on the complexity of the WDSs analysed. The results obtained are also compared to the results reported or obtained by using a number of reviewed pressure-driven model (PDM) methodologies, or obtained by the demand-driven model (DDM) method where direct comparison was not possible, with the aim of assessing the overall performance and convergence of the impact assessment model.

In Chapter 5 the demonstration and verification of the methodology presented in Chapter 3 at reducing negative consequences and identifying near real-time optimal recovery interventions is tested on a real water distribution network of DTown. The network description, data, assumptions, software used, and hypothetical failure-event scenarios, are given. Scenarios involve a medium burst on main pipe supplying a DMA and a large burst on a critical supply path in the network. The results obtained from both scenarios are also compared to the results obtained by using the Enumeration method with the aim of assessing the overall performance of the response methodology, and the relevant discussions and conclusions are drawn.

In Chapter 6 the key findings of this thesis are summarized and relevant conclusions are drawn. The novel aspects introduced in this thesis are highlighted, followed by recommendations for further research. 


\section{CHAPTER 2: LITERATURE REVIEW}

\subsection{Introduction}

This chapter provides a literature review of the core topics and research that is of the relevance to the work project presented in this thesis. This is done with the aim to identify the current state-of- the-art in the field and justify the work performed within the thesis. The main covered topics include response frameworks to failure events, impact models and assessment, optimization methods in WDSs operation/operational interventions. This the chapter is organized as follows.

In section 2.2, the overall concept of responding to WDS failures is given. This starts with a comprehensive review of hazards, resulting failures and their impacts on the WDS which are discussed in the two first subsections. The best practice for responding to and managing the failure event and their corresponding generated impacts in WDSs are reviewed. Then a review for the common interventions (focusing more on network interventions) that are used to respond to failure events in WDSs are discussed. This followed by a review for some exist methods on WDS segments identification. Finally, some existed intervention selection approaches and their issues are reviewed.

In section 2.3, the approaches for modelling and assessing the impact of failure events and interventions are reviewed. First, some important performance indicators that have been considered in previous studies for quantifying the impacts and interventions are briefly reviewed. Next, the methods used to model failure events specifically pipe burst and interventions in WDSs are presented. Then, methods used to quantify impacts, specifically supply interruptions and pressure drops, in WDSs are reviewed and discussed. The approaches that used to solve the pressure-deficient issues in DDM hydraulic solvers are then reviewed in details. Finally, the concept of real-time modelling and some of its applications are discussed and given.

In section 2.4, a brief methods for optimising the WDS operation/operational interventions including related criteria and optimisation techniques are presented.

The whole literature review is summarised in section 2.5. 


\subsection{Response to WDS Failures}

Water distribution systems (WDSs) are one of the critical infrastructures that deliver water to different consumers. Given their complex distributed topology which consist of thousands of interlinked elements (e.g., pipes, pumps, control valves, nodes) that are used to treat, store and distribute supply between sources and consumers. In addition to their interconnectivity with the other infrastructure systems, such as power supply and telecommunication, WDSs are exposed to a variety of hazards and resulting failures.

This section provides a review of existing response methodologies for dealing with hazards potentially affecting the WDS, the resulting failures and impacts and the interventions that can be used in the context.

\subsubsection{WDS hazards and failures}

Hazards in WDSs can be defined as any event with potential to reduce the degree to which the system delivers a defined level of service (Butler et al. 2017). Hazards can be classified in a different ways. The most two common ways used in the literature are as follows: (1) internal or external in terms of their origin; (2) chronic or acute, based on their rate of change. Therefore, each hazard can belong to one of the following subcategories: (i) external-chronic; (ii) externalacute; (iii) internal-chronic; and (v) internal-acute. The details of these subcategories are summarized in Table 2.1.

External hazards involve the effect of any external entity, force or factor, for instance natural, water consumers, and water utilities operators. On the other hand, internal denotes a hazard that originates with the water service provider or water infrastructure, such as a lack of investment or a poor maintenance regime. Chronic hazards are those that occur regularly and gradually in expected or predictable prospective over the time such as population growth, and ageing of infrastructure elements (e.g., pipes). Finally, acute hazards are those that occur suddenly and quickly (e.g., earthquake), and are usually unexpected, unplanned and/or unpredictable. 
Table 2.1 Potential hazards and their classification in WDSs.

\begin{tabular}{|c|c|c|}
\hline & Chronic & Acute \\
\hline Internal & $\begin{array}{l}\text { - Insufficient rehabilitation } \\
\text { - Resources depletion } \\
\text { - Loss of collective skills and } \\
\text { knowledge } \\
\text { - Incremental innovation } \\
\text { - Lack of investment }\end{array}$ & $\begin{array}{l}\text { - Insufficient maintenance } \\
\text { - Accident } \\
\text { - Human and technical errors } \\
\text { - Poor management }\end{array}$ \\
\hline External & $\begin{array}{l}\text { - Climate Change } \\
\text { - Urban creep } \\
\text { - Population growth } \\
\text { - Infrastructure ageing } \\
\text { - Stringent regulation } \\
\text { - Demand variation } \\
\text { - Demographic change }\end{array}$ & $\begin{array}{l}\text { - Natural Disasters } \\
\checkmark \text { Earthquakes } \\
\checkmark \text { Floods } \\
\checkmark \text { Hurricanes } \\
\checkmark \text { Tornados } \\
\checkmark \text { Tsunamis } \\
\checkmark \text { Wildfires } \\
\text { - Extreme weather } \\
\checkmark \text { Drought } \\
\checkmark \text { Floods } \\
\checkmark \text { Winter storms } \\
\text { - Power outage } \\
\text { - Terrorist attacks } \\
\text { - Cyber attacks }\end{array}$ \\
\hline
\end{tabular}

Because of the hazard, the concept of middle states (Johansson 2010) occur in the system and include all the possible modes of failure event for a given WDS. A good and popular example for middle state in WDSs is pipe break and leaks, which may occur as a result of various hazards (e.g., earthquake). Middle status can be divided to operational (functional) or structural (mechanical) fails in WDS. Pipe failures (e.g., breaks and leaks), pump failure, control valve failure, and other equipment failures (e.g., sensors, actuators), in addition to power system failure are examples for structural fails. Whilst, increased demand, pressure increased (i.e., because of water hammer), contamination, biofilms growth, internal pipe corrosion, and chemical reaction are consider as functional fails. This thesis focuses on mechanical failures such as pipe bursts. 


\subsubsection{Impacts of failures on the WDS}

The negative influences that the hazards and the resulting failures have on the expected level of service of a WDS are referred to as impacts (Butler et al. 2017; Cabinet Office 2011; IPCC 2012). Expected levels of service may be based on local, national, international, or global level standards and are used by regulators to monitor the quality of service provided. For example In the UK, the high standard of delivery of potable water is monitored by the Water Services Regulation Authority (i.e., OFWAT) using a range of performance indicators, which, amongst other aspects, evaluate the long term pressure adequacy (i.e., pressure of water mains, the DG2 indicator) and continuity of water supply (i.e., supply interruptions, the DG3 indicator) (OFWAT 2013, 2018c).

The magnitude and scale of the impacts depends on a number of factors such as geographic location and topology of a given WDS. Although impacts can be understood in many different ways, the present work uses this term to define a disturbance on the level of service or the performance of WDS. Impacts can therefore be categorised depending on the level of service or performance attribute being disturbed, whether these are related to hydraulic performance (e.g., water supply interruption, drops in pressures), quality performance (e.g., water contamination, discolouration of the water), environmental performance (e.g., pollution impact), efficiency performance (e.g., energy use impact), economical performance (i.e., costs),etc. Impacts have been used as a measure of the operational performance (i.e., through indicators) of the WDS under different abnormal conditions.

When impacts affecting the performance of a system are transferred to the broader socio-economic framework, this is referred to as consequences (Misiunas 2005; Casal 2016). Thereby, consequences are considered as the wider implications of impacts to society, the economy and the environment. Indeed, a pipe burst event, aside from an operational impact triggered by a failure of the WDS, may involve various negative consequences related to social (e.g., personal psychological damage, human lives), economic (e.g., loss of revenue, repair costs, material damage and disruption), traffic disruption, environmental issues (e.g., ecosystem or habitat losses) an other social consequences such as loss of public confidence and reduced workforce. 
The work in this thesis focuses on the hydraulic type failures impacts, specifically supply interruptions and pressure drops. Further, the impact assessment model developed in chapter 3 is only used to capture these two components and to evaluate the alternative interventions. However, the model is generic and more impact can be add to it if require.

\subsubsection{WDS failure event management}

The reliability and risk analysis has been widely used as the primary criterion in designing, operating and managing WDSs (Farmani et al. 2006; Kanakoudis and Tsitsifi 2001; Islam et al. 213; Gheisi et al. 2016). Traditional risk-based methods have been conducted to guide protection and prevention options that seek to mitigate or limit the likelihood of expected failure events and the potential negative impact from the failure events (Kanakoudis 2004; Kanakoudis and Tolikas 2004; Blakmore and Plant 2008). These options can enhance water systems' reliability to some extent and be helpful to prevent undesirable consequences.

However, recent experience from natural and man-made water-related hazard, as shown in section 2.1.1, suggests that existing WDS cannot protect and prevent all disruptive events and may perform unreliably because of high uncertainty of disturbances, complicated interdependency of systems, and stochastic failures resulting from unplanned failure events such as pipe burst (Little 2002; Asefa et al. 2014; Butler et al. 2017; Amarasinghe et al. 2016; Shin et al. 2018). Thus, interruption to water services, low water pressure conditions, and water discoloration are commonly observed inevitable problems. These problems could arise as results of either planned interruptions such as periodic maintenance and system rehabilitation or due to unexpected events, such as pipe burst and equipment failures (Bicik et al. 2009; Turner et al. 2012; Cook et al. 2016; Diao et al. 2016). This, in turn, may cause undesirable disruptions to consumers, utilities, and the environment (Vamvakeridou-Lyroudia et al. 2011; Rasekh and Brumbelow 2015; Nayak and Turnquist 2016). In such failure condition, proactive, economical and fast management action is essential to protect network from adverse impacts and to restore the service to normal operating condition.

In general, a good failure management plan in a WDS comprises two main phases (Boulos and Wiley 2013; Laucelli and Giustolisi 2015; Butler et al. 2017): 
1. Pre-event phase, aimed at lowering the probabilities of a failure event occurring in the first place or minimizing its possible effects before disruptive failure event occurs. This is done through improving strategies such as replacing/rehabilitating critical pipes/equipment or adding redundancy to a WDS. In general this phase include two components: (1) mitigation or prevention (e.g., appropriate system design and operation, improved organisation, risk analysis and information management) and (2) preparedness (e.g., improved organisation, risk analysis and information management).

2. Post-event phase which involves the following three components: (1) realtime detection/alarm system, where data (e.g., pressure and flow) are collected from monitoring equipment such as supervisory control and data acquisition (SCADA), smart meters and network sensors, and then analysed to visualize and detect the source of failure such as pipe burst; (2) response, limiting the extent of loss (e.g., real-time control of the system to identify the source of the disruption, interventions to isolate the incident, minimize the resulting impacts for consumers, reduce the magnitude and duration of the failure mode, and trying to restore the normal functionality of the system); and (3) recovery which aims to return to a normal state (e.g., repair/ replace for pipe bursts and other damaged network equipment, decontamination for water quality failures, etc.).

As described by Bruneau et al. (2003) and O'Rourke (2007), pre-event phase is affected by robustness and redundancy. The robustness is defined as the level of system service before any actions are taken. Moreover, robustness is more related to the design and the initial capacity of the system and its ability to perform under stressful conditions without failure. System redundancy in WDS can be described as alternative paths, such as multiple loops or options, and diverse sets of pumps. Post-event phase, on other hand, is affected by resourcefulness, and rapidity. Resourcefulness is defined as the necessary resources and services to detect a pipe burst, reduce generated negative impacts and repair the failure event. Whilst, the rapidity is the speed with which the interruption can be overcome, such as detecting, isolating, and repairing the component failure. 
Critical Infrastructure Partnership Advisory Council (2009); American National Standards Institute (2010); US Environmental Protection Agency (2011, 2014, 2015, 2016); OFWAT (2013, 2018a, 2018b, 2018c) and Drinking Water Inspectorate (2011) provide general guidelines for network operators about how to respond to an emergency situation in WDSs, they do not provide a decision support tool that can help the network operators do their job even better by recommending specific actions to be taken in near real-time especially in response phase.

According to the Planning for an Emergency Water Supply (US Environmental Protection Agency 2011) regulations, each WDS that serves more than 3,300 habitants is mandated to prepare vulnerability assessment and emergency response plans within a specified timeframe for situations when a failure event is confirmed. The response plan should involve a rapid identification and quantification of system losses and impacts (i.e., risk assessment, notify affected consumers). This is followed by defining a number of temporary corrective and remediation intervention actions for network operators as an emergency response to limit the potential consequences of failure and to return to pre-failure operations conditions, e.g., flows and pressures (Bicik et al. 2010; Laucelli and Giustolisi 2015).

Boulos et al. (2014) declares that having a robust real-time response plan will have a number of benefits for water utilities such as reduce overall risk through hazard planning, increase system availability, decrease downtime and ensure business continuity. Also, consumers can benefit from the response plan through reduce outage duration, increase awareness of restoration progress and time estimates and increase consumers safety.

The methodology adopted in this thesis focuses on the second stage of the postevent phase, i.e., response. The methodology is based on the current practice in the UK water industry and it is intended to provide support to network operators when making relevant decisions, i.e., help them do their job even better, especially in cases when non-catastrophic failures are affecting a larger part of the water distribution system (please see chapter 3 ). 


\subsubsection{Interventions}

Definitions and applications of the term "intervention" in the literature are varied, but intervention is typically considered to entail targeted actions or adjustments carried out in a specific system in response to actual or anticipated hazard in order, to minimize or prevent negative impacts (e.g., Jones and Preston 2011; IPCC 2012). Thus, intervention here is defined explicitly as any action taken to modify specific properties of the WDS to enhance its capability to maintain levels of service (e.g., pressure, nodal outflow) under varying conditions after failure event occurrence in response phase. This in turn can increase reliability, enhance resilience, and/or improve sustainability (Grothmann and Reusswig 2006) of the system.

The operational intervention in the literature involve, for example, isolating failure event location (Jun and Loganathan 2007; Giustolisi and Savic 2010), providing alternative water supply options (Turner et al. 2012; Nayak and Turnquist 2016), redistributing network flows (Fujiwara and Li 1998) or readjusting of flow patterns (Bouchart and Goulter 1991). All with the aim to improve flow conditions (e.g. pressure and quality) in the affected area and increase system resilience and reliability under failure conditions. There other types of interventions actions could be potentially used to provide affected consumers with water, e.g., via water truck or delivery of bottled water, etc. and both are used popularly worldwide. However, this review section and thesis focuses on network interventions only. This thesis proposes three type operational interventions include (i) open/close suitable isolation valves; (ii) installing overland bypasses between two suitable hydrant points; and (iii) modifying pressure settings at the end of available PRVs (further detail shown in chapter 3 ). These interventions are used to improve flow and pressure conditions under failure events such as pipe bursts.

For example, Jeong et al. (2006) developed an approach to provide water only to critical consumers (e.g., hospitals) while the WDS is only partially functional due to natural disaster such as earthquake, and this lead to the reduction in the negative impacts. Later Jeong and Abraham (2009) tried to improve upon the previous study by developing a scheduling model to supply water to each consumer temporarily during some time of the day, hence all network consumers can receive some water. The contribution of Turner et al. (2012) and Nayak and Turnquist (2016) focused on identifying unaffected consumers (i.e., required 
demands fully satisfied) after a failure event occur, and then supply water by using distribution trucks to affected consumers (e.g., those that cannot receive any water). However, the aforementioned approaches exhibit one or more of the following limitations for wider implementation: (i) assumed static water demand patterns for water consumers; (ii) nodal head flow relationship is ignored, in which nodal outflow may be reduced due to reduction in pressure head; (iii) isolation of segments conducted regardless of valve locations in the network; and (v) offline models (driven by collected and historic data sets, i.e., without connection to any real-time source of data such as SCADA).

Vamvakeridou-Lyroudia et al. (2011) proposed a hierarchy structure algorithm to reduce the negative impacts of a single pipe burst. For every possible pipe event scenario in the network, a unique offline list of interventions is produced based on the historical data of flow and pressure. When the failure event is detected and located, the relevant response interventions are retrieved from this list and are conducted as real-time response solutions. However, this approach seems too rigid and not generic enough to provide network operators with enough flexibility when selecting intervention options.

Zhuang et al. (2013) introduced an adaptive pump and valve operation methodology (non-optimization-based method) to mitigate the hydraulic effect of pipe failures in a WDS. Their method identifies the best set of pumping units with various capacities after isolating the affected subsection of the system. Zhuang et al. concluded that having multiple pumps of various capacities increases the system water resilience/ availability under failure conditions. This conclusion was also summarized by Lansey (2012), who described multiple pumps as an example of system redundancy. However, this approach is unable to provide water supply to unintentionally disconnected consumers (i.e., consumers that are disconnected from the water sources as a result of isolating the event in the pipe network).

Morosini et al. (2016) developed a response methodology based on the nodal demand control, with the aim to increase the pressure head, and hence the flow rate actually delivered at critical nodes (i.e., hospitals, vulnerable customers, etc.). This was done to avoid or minimize service interruptions between the failure and the repair times, due to a pipe burst isolation which cause a portion of the 
network faced to reduction of head in some nodes. However, applying such methodology on a large WDS would be a difficult task.

On the other hand, evolutionary algorithms such as non-dominated sorting genetic algorithm (NSGA-II), decision trees, and heuristic methods have been used for making better decisions to protect public health against contamination events (Preis et al. 2007; Poulin et al. 2008; Baranowski and Leboeuf 2008 ; Preis and Ostfeld 2008; Alfonso et al. 2010; Poulin et al. 2010; Alvisi et al. 2012; Rasekh and Brumbelow 2014, 2015; Rasekh et al. 2014). Flushing suitable fire hydrants and manipulating valves were considered as response actions to alleviate the consequences in these studies. However, most of these approaches are offline models and the problem of interest presented in this thesis refers to different types of unplanned failure events such as pipe bursts.

\subsubsection{Intervention selection approaches}

The selection and implementation process of intervention actions should be accurate and quick as the wrong or slow decisions could make the situation worse. However, this relies on the current configuration of the existing WDS in the affected area, the accessibility of alternative interventions for providing the water, and the availability of valve control options for isolating affected areas and rerouting water (Tian et al. 2007; Vamvakeridou-Lyroudia et al. 2011). Additionally, performing this in a timely manner is difficult because of the uncertainty, complex hydraulic and water quality behaviour in WDSs under failure operating conditions (Rasekh and Brumbelow 2015). Clearly, the availability of information about current operation conditions in a particular situation such as pipe burst on real-time basis is crucial for decision making and response models in WDSs. For example, demand management is a dynamic process, changing hourly, daily, weekly or monthly depending on customer requirements (Romano et al. 2013). Also, Walski (2015) stated the models used in emergency situation must be accurate and trusted, as misleading results may be more dangerous than no models or action taken at all. However, because situations differ so widely from one case to another, it is impossible to develop rigid rules for acceptability of models. 
Hydraulic and water quality network models such as EPANET combined with mathematical programming methods, such as for example artificial neural networks, heuristic optimization and genetic algorithms, have been extensively used in WDSs (e.g., Prasad and Park 2004; Boulos and Wiley 2013; Fu et al. 2013). These models help network operators to predict the WDS behaviour under different operational conditions, improve the performance and ensure more reliable operations at minimum cost and in near real-time. For example, operators can quickly assess the consequences of a sudden pipe burst or other equipment failure in a specific WDS (Walski 2015), as well as generate near-optimal solutions for enhancing system operation. However, few models or systematic algorithms exist to support the response and management process of failure consequences. Indeed, to date, most models and related studies in the field of response to failure events have been limited to primarily identifying the location of the failure event (Laucelli et al. 2016), vulnerability analysis (Laucelli and Giustolisi 2015), strategic reliability (Bouchart and Goulter 1991; Fujiwara and Li 1998; Giustolisi et al. 2008), unaccounted-for (non-revenue) water reduction (Kamani et al. 2012), pipe deterioration modelling (Osman 2010), early contamination warning (Ostfeld et al. 2004), pipe replacement/rehabilitation (Yoo et al. 2014), event detection systems (Romano et al. 2014), pump energy cost optimization (Sunela 2017) and leakage management (Berardi et al. 2015), and risk analysis (Bicik et al. 2009; Seth et al. 2016). Where limited studies have focused on the elaboration of a timely intervention strategies in the field after a failure event (i.e., pipe burst) has been detected and located.

On the other hand, mathematical programming methods are able to produce good quality solutions but computationally inefficient, especially in analysing/designing large WDS (Zheng et al. 2013; Maier et al. 2014). Searching for near optimum solutions instead of trying to find all possible best optimal solutions is a way to address this issue (Gibbs et al. 2015). Alternatively, graphical method (Kadu et al. 2008), network decomposition algorithm (Zheng et al. 2011, 2013), and heuristic method (Jayaram and Srinivasan 2008; Poulin et al. 2008) have been used to reduce the global search space, as well as computational time for the optimal design and operation issues in WDSs. But even with this reduction, the overall computational time is still substantial for analysing WDS in near real-time (i.e., greater than one hour) the further detail of this methods are already discussed in section 2.4. 


\subsubsection{Segment identification in WDSs}

The partitioning of WDSs into segments and district metered areas (DMAs) provides various management benefits for system operators. These benefits include, for instance, improved pressure management, more efficient leakage control and effective isolation of network parts in cases of pipe breakages. All this, in turn, increases system reliability and resilience. Identification of network segments depends mainly on the distribution and location of valves in a given WDS. In general, valves are used for controlling flow conditions (i.e., pressure and flow) and for isolating a failure area due to pipe breakage failure or contamination events.

Typically, the distribution of isolation valves is followed general rules of thumb such as locating at least three isolation valves at cross-sections (i.e., junctions) and two valves at each $\mathrm{T}$-intersection (i.e., $\mathrm{N}-1$ rule), or the $\mathrm{N}$ valve rule, consisting of installing isolation valves in all of the adjacent pipes of each junction (Jun and Loganathan 2007; Jun et al. 2008). This in turn will reduce the sizes of the isolated segments (Mays 2000). However, in terms of budget limitation, installation/maintenance fees, and increased risk of leaking water at cracks, the $\mathrm{N}-1$ and the $\mathrm{N}$ valve placement rules are not followed always. Walski (1993) defines a segment as the portion of the network that can be isolated by closing valves to contain the damaged pipe. A segment will be a single pipe, only if, that pipe has two valves located at its both ends. Otherwise, isolation of a pipe will require closing valves on other pipes and nodes; thus the resulted segment will be comprised of pipes and nodes bounded by isolation valves. Jun and Logathatan (2008) claims that as a result of regular segment isolation, other portion (i.e., pipes and nodes) of the network may unintentionally isolated from the available water source(s) in the network. How to optimize a valve system to achieve reasonable segments and minimal isolated segments is a crucial topic in the field of reliability research in WDNs (Creaco et al. 2012; Walski 2011).

A number of methods for segment identification in WDSs are available in the literature. For example, Jun and Loganathan (2007) introduced matrices to present the topological relationships between the nodes and links of a WDS and used a depth-first search-based algorithm to identify segments. Also in their methodology, an unintended isolation algorithm was proposed to identify the unexpected shut-off areas for closing a critical segment. In each identification 
step, the depth-first search-based algorithm traverse all connectable nodes from water sources before an unintended isolation can be identified. If all critical segments in a WDS are desired, the approach should be performed the same times as the number of segments in a network. The proposed algorithm was able to effectively identify all critical segments in a single hydraulic run.

Kao and $\mathrm{Li} \mathrm{(2007)} \mathrm{and} \mathrm{Li}$ and Kao (2008) use a topologic matrix and a deep search algorithm in segment identification and vulnerability analysis. Kao and Li (2007) consider hydraulic characteristics in the analysis of unintended segments. The links connected to an arbitrary node are first identified and stored and if one of the components is a valve, it is marked as a boundary for the segment. The connected nodes and pipes are then stored as a new segment and identified as being "visited". Then, the process is repeated using a new unvisited node until the entire network is examined. Once the segments have been identified, the computer program uses an algorithm based on the articulation point identification method (Li and Kao 2008) to detect the critical segments, or the segments that block the water supply downstream.

Giustolisi and Savic (2008) regard valves as pseudo-pipes and solve pressure driven equations to identify segments. Recently, Giustolisi and Savic (2010) have improved their method by using a valve topological matrix and computing an incidence matrix of power to the pipes to identify all pipe transitive closure sets (Even 2011), i.e., segments in a single hydraulic run. Also the Giustolisi and Savic (2010) use the incomplete Cholesky factorisation (Zhang 1996), instead of the pseudoinverse matrix (Penrose 1955), for the simplified hydraulic simulations aimed at the detection of the undesired disconnections (i.e., unintentional segments).

Similarly, by using topological matrices and assuming valves as fictitious pipelines, Creaco et al. (2010) compute node transitive closures by moving fictitious water sources and solving hydraulic equations to identify segments one by one. Instead of using fictitious pipelines, Alvisi et al. (2011) use an auxiliary valve matrix and vector to model valves and identify segments one by one. They also analyse the hydraulic impacts of equipment such as check valves, pumps, and PRVS on unintended segments by modifying a node-to-node connectivity matrix. 
Kaldenbach and Ormsbee (2012) proposed a simple method to identify intended segments based on a node-node connectivity matrix in which valves are also treated as nodes. The method also proceeds to list the unintended isolations in the network and the impacts of each failed segment. Di Nardo et al. (2013) proposed a methodology uses graph theory principles and a heuristic procedure based on minimizing the amount of dissipated power in the water network to identify segments.

Gao (2014) used an algorithm based on the theory of transitive closure of graphs. The algorithm models real valves as fictitious valves, uses valve pairs to simulate valve on-off status, and improves the Warshall algorithm to compute node transitive closure sets to identify all network segments at a time. In addition to that, the algorithm was able to identify, redundant valves, endpoint valves, leaf segments, orphan segments, and regular segments. Also, one-way valves are considered in an unintended segment analysis.

In this thesis, a methodology is proposed to identify network segments (i.e., sets of pipes and nodes) that belong to an individual valve or a set of valves. The methodology is developed based on the basic network topological matrices (i.e., node-link connectivity matrix, valves topological matrix). In comparison to other existing methods, the proposed methodology has able to produce all segments for a given WDS in single network run without introducing pseudo valves, pipes and auxiliary valve matrix or performing hydraulic analysis. Additionally, it is very simple for computer coding and does not require the user to have extensive programming knowledge. The detail of the segmentation methodology is shown in section 3.3.1.

\subsection{Impact Modelling}

\subsubsection{Operational performance assessment}

The performance indicators or metrics of WDSs have been widely studied in the literature with the goal of assessing the system's operational performance before, after and during the failure event. Also, they are used to identify the best way to respond to failure event and restore the normal functionality of a WDS before the event. Moreover, the performance indicators are measures used to describe the behaviour of the WDS under abnormal conditions in terms of its tangible 
operational properties. The indicators and metrics are used interchangeably in thesis to indicate to measure for quantifying the impacts.

In general, for WDS performance, the indicators can be categorised into topographic, hydraulic, water quality/security, greenhouse emissions, social welfare, and economic (i.e., cost). These performance indicators are typically used in the development of the implicit measures of risk, reliability, resilience, robustness, and redundancy analysis. For a WDS and in consumers' point of view, the performance indicators predict the system behaviour primarily based on hydraulic/quality properties in term of demand node outflows, available pressure head at consumers taps, supply interruptions and water quality problems. This section presents several popular hydraulic key performance indicators. A full overview of aforementioned metrics especially hydraulic metrics can be found in Ostfeld (2004); Mansoor (2007); USEPA (2014b); Gunawan et al. (2017); and Shin et al. (2017).

Hydraulic metrics are derived based upon spatially and temporally variable flows and/or pressure; calculation of these metrics require simulation of WDS hydraulics that reflect how the system operates under normal and emergency conditions. Reliable networks must be able to deliver required flows to consumers at adequate pressure during these emergency conditions; however, not all hydraulic reliability metrics explicitly consider all of these conditions. While some hydraulic reliability metrics are calculated over a time interval, others are calculated using flows and pressures at a single time.

Su et al. (1987); Wagner et al. (1988); Bao and Mays (1990); Fujiwara and Ganesharajah (1993); Ostfeld (2001); and Ostfeld et al. (2002) use stochastic simulation to analyze reliability in WDS networks. With stochastic simulation, an ensemble of hydraulic scenarios can be defined by sampling from probability distributions of, for example, demand profiles, initial water quality, the time and location of pipe breaks, and the time it takes to repair individual components. This helps to estimate the reliability and resilience of a WDS to a wide variety of conditions.

Ostfeld et al. (2002) developed an approach which uses stochastic EPANET simulations and computes the fraction of delivered volume (FDV), and fraction of delivered demand (FDD).To be able to more accurately calculate demand under 
failure scenarios, modelled demands were corrected based on the pressure and flow rate. In this way, a node is only supplied its fully requested demand when a minimum pressure constraint is met, otherwise only a fraction of the demand is satisfied. FDV is the ratio of total volume delivered to the total volume requested, and FDD is the fraction of time periods where demand is met at each network node. Similarly, Ozger (2003) measure available demand fraction (ADF) using a pressure dependent correction of EPANET hydraulic simulations.

Awumah and Goulter (1989) compute the percentage of demand supplied at adequate pressure (PSPF). This metric requires a hydraulic simulation for each pipe removal in the system. For each simulation, the fraction of demand that is supplied when pressure is above a specified threshold is recorded. Wagner et al. (1988) also measure the number and duration of failure event like pipe failures, the number and duration of reduced service events, and the between failure time and repair duration.

Zhuang et al. (2013) described the concept of "availability" as system resilience of a water distribution network, which is defined as the fraction of water supplied to required demand nodes over the time involving the failure event period. Shuang et al. (2013) defined system hydraulic metrics as the probability that water is successfully supplied to consumers allocated to nodes during a failure period. Clearly, it is expressed as the ratio of the total actual water demand versus the total required water demand given different failures.

Todini (2000) developed a resilience indicator for the looped water distribution networks to cope with uncertainties in failure events. The physical and hydraulic failures (e.g., pipe breakage) in the WDS may entail more internal energy dissipation with variation of the water flow and pressure. He considered that the resilience of a water distribution network depends on sufficient energy surplus to overcome increasing internal energy dissipation in the case of disruptions. The energy surplus represents the available energy storage that can be dissipated under changes in operation conditions by a failure event. In this regard, the proposed resilience indicator was defined as the fraction of the available energy surplus at the nodes over the maximum energy surplus in the network, which would be internally dissipated to meet the required demand and head at the nodes. 
Cimellaro et al. (2015) proposed a global resilience measure by combining social, technical, and environmental system performance. The social, technical, and environmental performance was defined as the functionality of each domain under failure event compared to their functionality under normal operating conditions. The performance is estimated by use of the ratio of the numbers of households satisfying water requirements after failure event over a total number of households for the social domain, the ratio of the tank water level over the level corresponding to the tank reservoir capacity during a control time period for the technical domain, and the ratio of water quality over the pre- and post-failure event for the environmental domain.

Supply Interruption Scale (SIS) and Supply Interruption Duration (SID) indicators have been developed by OFWAT $(2018 \mathrm{a}$, b) to estimate supply interruption, which defined as the number of consumers disconnected from the water supply or a situation when no water is available at the consumers' tap (i.e., the pressure is below the minimum pressure threshold $P_{\text {min }}$ ), due to failure event in WDS, and lasting longer than 3 hours, 6 hours, 12 hours and 24 hours. Also, OFWAT (2018a, b) developed Scale of the Low Pressure (LPS) indicator to calculated the total number of consumers receiving substandard pressure (i.e., the pressure is between the minimum pressure $\left(P_{\min }\right)$ and required pressure ( $\left.P_{\text {req }}\right)$ thresholds) for more than 1 hour throughout the whole day.

It can be seen from the above that a number of performance metrics exist in literature to capture the supply interruption and pressure conditions in WDS. The pros and cos of each metric can be found in the above papers. The metrics of Zhuang et al. (2013) were applied in this thesis to evaluate the performance of the developed PDM (see chapter 3). Also, these and two newly developed metrics shown in chapter 3 are used to estimate the impact associated with a pipe burst (and to test the performance of the proposed interventions in this thesis).

\subsubsection{Modelling of failure events in a WDS}

Modelling of failure event especially pipe failure (e.g., burst and leak) has received significant attention in the literature, primarily due to its important rule in reliability, resilience studies and risk assessment. This section provides details 
regarding the modelling of failure event in the hydraulic solver in particular EPANET (Rossman 2000a). Based on the purpose of the simulation and the capabilities of the hydraulic solver used, the methods of modelling pipe failure can be broadly divided into: (1) strategic or (2) operational applications.

In strategic applications, the time over which a pipe/equipment needs to be isolated for repairs is dominant and, therefore, the outflow from a burst in case of pipe before the isolation takes place is neglected. The simplest technique applied by several researchers (e.g., Farmani et al. 2005) is to disconnect the failed pipe from the network. In EPANET a single pipe/equipment can be disconnected by either (i) setting the status of pipe/equipment to CLOSED, (ii) setting the diameter (in case of pipe) to very small value (e.g., 0.0001 ) or (iii) physically removing the pipe/equipment from the system. The last option, although being the most difficult one to implement, can be considered as the best way since it effectively reduces the complexity of the governing nonlinear equations and thus speeds up the convergence of the global gradient algorithm (Todini and Pilati 1988) used in EPANET. It also eliminates potential convergence problems caused by the other two approaches, which introduce abnormally high resistance coefficients in the hydraulic equations.

In operational applications, the failure event such as pipe burst can cause large changes in system hydraulic conditions (e.g., drops in pressures, supply interruptions). Operational method aims to explicitly model the water lost due to a burst/leak between the time when it starts and the time when repair crews can isolate the leak/burst by using nearest set of available isolation valves. Hayuti et al. (2008); and Mansoor and Vairavamoorthy (2003) modelled pipe bursts in EPANET by inserting an artificial reservoir in the middle of a pipe and setting its water surface level to correspond to the elevation of the pipe. The outflow through the pipe was then controlled by changing its properties (i.e., diameter, length and roughness). Placing the burst into the centre of a pipe is an approximation which is reasonable for relatively short pipes, however, might become less applicable in rural areas where pipe lengths tend to be significantly longer than in urban areas.

More frequently, pressure sensitive outflows are modelled using emitters, which are devices used typically to model sprinklers or irrigation networks governed by the equation for orifice flow (Walski et al. 2003): 


$$
Q=C_{d} A \sqrt{2 g h}
$$

Where: $Q$ is the outflow (discharge), $C_{d}$ is a discharge coefficient, $A$ is the area of an orifice, $g$ is the gravitational acceleration constant $\left(9.81 \mathrm{~ms}^{-2}\right)$, and $h$ depth of water measured from the centre of the orifice opening $(m)$.

The orifice equation can be generalised and written as:

$$
Q=C_{d} P^{r}
$$

Where: $Q$ is the flow rate, $P$ is pressure at junction, $C_{d}$ is a discharge coefficient and $y$ is a pressure exponent.

Studying the behaviour of bursts and leakage has attracted the attention of many researchers. Van Zyl and Clayton (2005, 2007); and Klise et al. (2017) investigated that leak hydraulics, pipe material behaviour, soil hydraulics and water demand are the main factors affecting magnitude of pipe a leak/ burst. Van Zyl and Clayton (2007) further noted that specific types of failures are likely to develop depending on pipe material. Results of their experimental study related pipe material and type of the opening to the pressure exponent $\gamma$ which had the most significant effect on the flow through a burst. The value of $y$ typically ranged from 0.52 to 1.85 for round holes and longitudinal cracks, respectively. Lambert (2002) reported that values of $y$ typically ranged from 0.5 to 1.5 and occasionally also between 2.0 and 2.5 during field tests conducted in the UK. Lately, Cassa et al. (2010) conducted a numerical study into the effects of pressure on holes and cracks and concluded that values of $\gamma>1.5$ did not have theoretical justification. In the work presented here, the approach of Klise et al. (2017) was adopted to simulate pipe break scenarios. Where, break at pipe is simulated by adding a dummy pipe (with large diameter and small length) and an emitter at the middle of the damaged pipe. The emitter coefficient is equal to $C_{b}=6.07{ }^{*} 10^{-5 *} d^{R}$ ( $d$ is the diameter of the pipe in $m m$ and $C_{b}$ is in $L / s / m^{0.5}$ ), while the exponent is $\gamma=0.5$. A new method was developed to simulate the proposed interventions in this thesis in hydraulic solver as shown in chapter 3, 4 and 5 . 


\subsubsection{Impact quantification}

Because of the different social aspects, an accurate estimation and quantification of impacts in WDS is highly subjective and complex problem. In this section, we will specifically focus on the methods and tools that can be used to quantify the impacts of events such as supply interruptions and pressure drops in the WDS. This is done because this is of direct interest to the work done in this thesis (see chapters 1 and 3 ).

Hydraulic and water quality network models represent the most effective, efficient and viable tools to predict WDS behaviour under a wide range of demand loading and operating conditions including emergency loading conditions resulting from different hazards as described in section 2.2.1. Mays (2000) defines emergency loading conditions that distribution networks are designed to handle on a routine basis: fire-fighting water demands, pipe breaks, sensor failures, pump failures, power outages, control valve failures, and insufficient storage capacities. The network models used to analyse the resilience of WDSs to hazards need to be able to robustly handle failures and emergency/ stresses on the system.

The hydraulic and water quality models use the laws of mass and energy conservation and reaction kinetics to determine pressure, flow, and water quality (movement and transformation) conditions for specified system characteristics and operating conditions (e.g., steady-state, EPS analysis). Through their predictive capabilities, these deterministic models provide a powerful tool for evaluating system response to various operational and management strategies to meet specific performance goals (Sunela 2017).

Most of the currently available hydraulic models that used for analysing WDSs have been traditionally derived by using demand-driven models (DDM) (Wood and Charles 1970; Isaacs and Mills 1980; Todini and Pilati 1988). These methods assume that the demand required will be delivered irrespective of the system pressures available. The DDM hydraulic models are based on the well-known mass and energy balance equations that are used to compute pipe flows and nodal pressures in the network.

These methods work well under normal flow conditions in which sufficient pressures are available in the pipe network. However, pressure can fall substantially under certain conditions, such as pipe bursts (or isolation) or during 
excessive water use (Wu and Walski 2006; Kapelan and Giustolisi 2006; Giustolisi et al. 2008). Under these flow conditions, DDM methods cannot always deliver realistic predictions of pressures and flows, because it is not always possible to deliver all desired demands in such circumstances. As a result, DDM methods may produce unrealistically low, sometimes negative nodal pressures that are, in some cases, physically impossible (Kapelan and Giustolisi 2006; Tanyimboh and Templeman 2010; Romano et al. 2014).

The pressure-driven models (PDM) address the aforementioned shortcomings by representing the water flow delivered as a function of available pressure (Bhave 1981; Germanopoulos 1985; Wagner et al. 1988; Chandapillai 1991; Fujiwara and Ganesharajah 1993; Gupta and Bhave 1996; Tanyimboh and Templeman 2010; Siew and Tanyimboh 2012). This is termed nodal head-flow relationship (NHFR). Having said this, some authors have proposed alternative approaches that do not make use of NHFR. Examples include (1) Collins et al. (1978), who proposed using optimization techniques instead; (2) Todini (2006), who developed a heuristic-type solution with three corrective steps to the demanddriven global gradient algorithm (GGA) (Todini and Pilati 1988), in order to correct nodal outflows whenever pressure is insufficient; and (3) Piller and Van Zyl (2007, 2009), who used mass balance-based constraints in the minimization of their content model.

Since the 1980s, many attempts have been made to simulate WDS under pressure-deficient conditions (Ozger and Mays 2003; Giustolisi et al. 2008; Wu et al. 2009; Tabesh et al. 2011; Siew and Tanyimboh 2012) and to formulate the NHFR. However, only a limited number of approaches succeeded in producing acceptable results under certain boundary conditions. Nevertheless, most of these approaches are not ideal, because they are either difficult to implement or computationally inefficient. This makes them difficult and sometimes impossible to use in larger networks, especially under extended period simulation (EPS) conditions (see the next section for details).

Probably the first NHFR function was suggested by Bhave (1981), who assumed that the actual (or available) nodal flow delivered $\left(Q_{n, d e l}\right)$ depends only on the minimum pressure head, $P_{n, \min }$, for flow to occur at node $n$, as shown in Figure 2.1. This work was extended by Germanopoulos (1985) to avoid the discontinuity properties in the aforementioned function. He proposed two characteristic head 
values for defining the NHFR, namely, $P_{n, \text { min }}$, the minimum head for flow to occur at node $n$ (where enough information is not available, the value of $P_{n, \min }$ is frequently considered equal to the elevation of node $\mathrm{n}$ ), and $P_{n, \text { req }}$, the head required to provide full flow, i.e., the demand required $\left(Q_{n, \text { req }}\right)$ at node $n$. The $P_{n, \text { req }}$ value for a node depends on the type of service connection and the type of development in the area served by that node. For instance, the pressure required at the street level for excellent flow to a three story building is about $290 \mathrm{kPa}$ (30 $\mathrm{m})$. The Office of Water Services in England specifies a minimum acceptable static pressure of $7 \mathrm{~m}(68.5 \mathrm{kPa})$ below which consumers may be entitled to compensation for less than satisfactory service. In general, nodal heads of 15 to $25 \mathrm{~m}$ will guarantee satisfactory service at all related stop taps in a distribution system (Tanyimboh et al. 1999). Salgado-Castro (1988) assumed a linear relation for the NHFR. Further, Wagner et al. (1988) proposed a continuous quadratic relationship for modeling partial flow between $P_{n, \min }$ and $P_{n, \text { req }}$ as follows:

$$
Q_{n, \text { del }}=Q_{n, \text { req }}\left(\frac{P_{n, \text { avl }}-P_{n, \text { in }}}{P_{n, \text { req }}-P_{n, \text { in }}}\right)^{1 / n_{n}} \quad \text {,if } P_{n, \text { req }}>P_{n, \text { avl }} \geq P_{n, \text { min }}
$$

Where $n_{n}$ is the empirical exponent coefficient at node $n$, where $n_{n}$ varies between 1.5 and 2 (Tabesh 1998), but Wagner et al. (1988) suggested $n_{n}=2$. Ackley et al. (2001) claim that the Wagner et al. (1988) function is hydraulically significant but not differentiable when outflow begins (i.e., $P_{n, \text { avl }}$ approaches $P_{n, \min }$ ) or when it

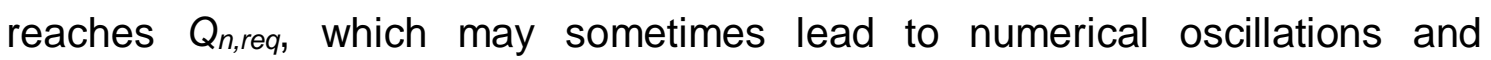
convergence problems in iterative methods that upgrade the NHFR in the GGA (Kovalenko et al. 2014). Thus, later, a differentiable function was suggested by Fujiwara and $\mathrm{Li}(1998)$ to simulate and assure a smooth transition for the partial flow between $P_{n, \min }$ and $P_{n, \text { req. }}$. 


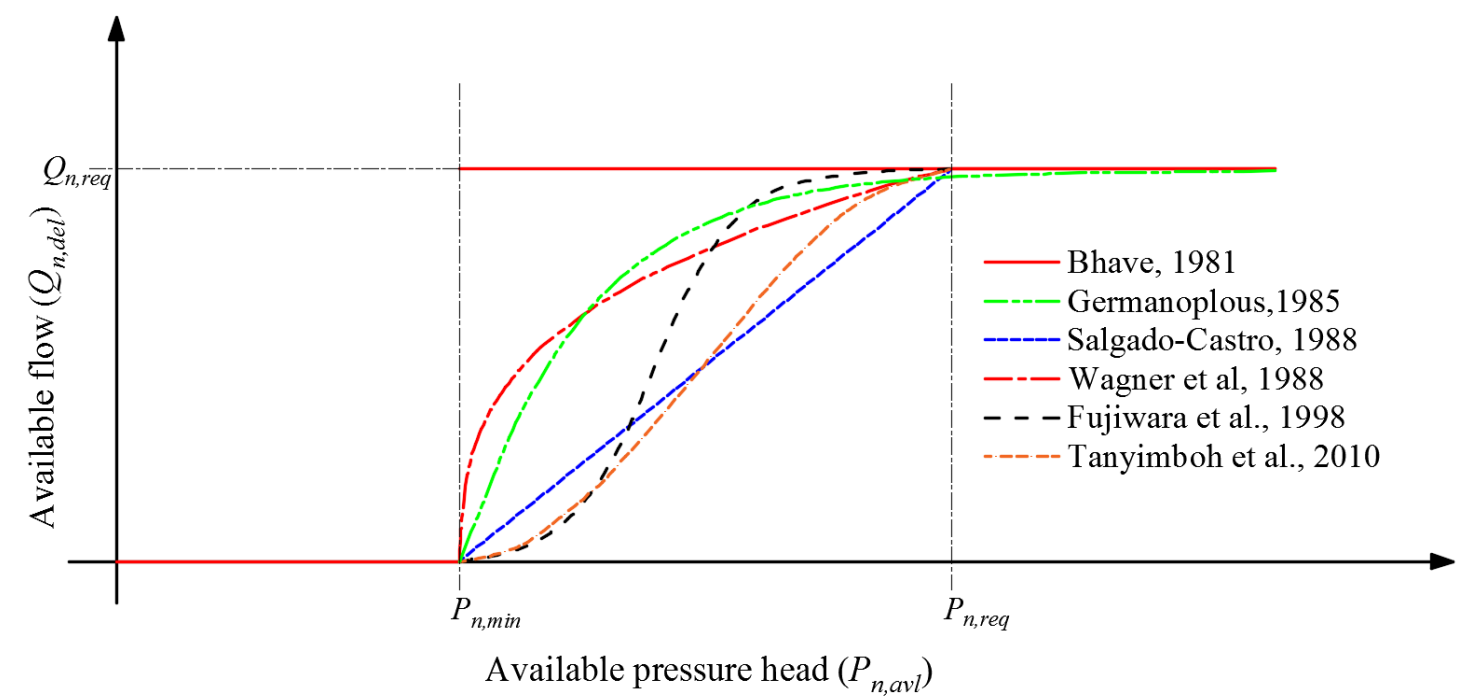

Figure 2.1. Comparison of different head-flow relationships used in PDM.

For the same reason Tanyimboh and Templeman (2010) proposed a logit function-based formulation to model partial flow. All the formulations mentioned for modeling the NHFR have been derived primarily on the basis of mathematical considerations and have not been validated by experimental or laboratory data. Also, Todini (2006) stated "it is impossible practically to derive a realistic NHFR." This is consists what Ciaponi and Creaco (2018) concluded: "only the comparison with experimental data can reveal which formula is the most consistent with the real WDS behaviour". However, the function proposed by Wagner et al. (1988) was highly recommended by Gupta and Bhave (1996), and Abdy Sayyed and Gupta (2013), among other existing functions at that time. Also, Todini (2006) states "it is impossible to drive a realistic NHFR". Furthermore, Shirzad et al. (2012) and Walski et al. (2017) investigated the performance of the existing NHFRs by comparing them to laboratory and field measurements collected from tap outlets under different pressure head scenarios. The NHFR functions presented by Wagner et al. (1988) matched the best experimental data. Kovalenko et al. (2014); and Vairagade et al. (2015) performed a further comparison between the Tanyimboh and Templeman (2010); and Wagner et al. (1988) functions for some networks and calibration parameters, and concluded that the Tanyimboh and Templeman (2010) function gives better convergence properties, especially when $P_{n, \text { avl }}$ approaches $P_{n, \text { min. }}$. 
Thus having a robust PDM with a reliable NHFR that is able to predict the hydraulic behaviour of a network under pressure deficient conditions in an accurate manner is essential for the work done in the thesis. Such a PDM does not currently exist in the literature. It is also noteworthy that the PDM presented in chapter 3 has the ability to model a more general NHFR and, therefore, even though the Wagner et al. (1988) function is used here (as recommended by several other authors) this is not a must, i.e., other NHFRs could be used as well.

\subsubsection{Approaches to model pressure-deficient conditions using DDM Hydraulic Solvers}

Most of the currently available WDN hydraulic solvers (except some commercial one e.g., WaterGEMS 8i) such as free open source EPANET2.0, WaterGEMS, and Mike, work as DDM-type solvers utilizing the GGA (Todini and Pilati 1988). Moreover, existing solvers were not designed to handle sudden failures resulting in inadequate pressure or rapid changes in system operation. Further, they were not designed to handle situations when sections of a water system become isolated because of a pipe or equipment failure, and tanks or reservoirs drain. However, the DDM-type hydraulic solvers (specifically EPANET2.0) can be used to analyze WDSs under pressure-deficient and emergency conditions as well, which can be done in two principal ways.

The first approach relies on using a specific pressure-demand relationship that requires changing the source code of the DDM simulator. A number of examples of this approach exist. Ackley et al. (2001); and Yoo et al. (2012) presented optimization methods that maximized nodal outflow under abnormal operating conditions. Although they delivered results for simple WDNs, no generic solution approach was developed for practical applications. More recently, Goldstein's algorithm was used by Elhay et al. (2015) to upgrade different NHFRs in the GGA (Todini and Pilati 1988). This approach was successfully demonstrated on eight challenging (i.e., one has 20,000 pipes) case study networks. However, this involved only steady-state hydraulic analysis. Rossman (2000b) extended EPANET by implementing a flow emitter at a node to simulate demand delivered as a function of available pressure. However, a flow emitter produces negative outflow (i.e., inflow) when nodal pressure becomes negative and there is no upper 
limit for the discharge value. Cheung et al. (2005) modified the source code of EPANET by introducing emitters into the network model to simulate pressuredriven demands by using an object-oriented toolkit (OOTEN). Yet this approach failed to converge when attempting to model highly looped WDNs under low flow conditions and EPS analysis. A modification of the emitter methodology was proposed by Morley and Tricarico (2014) through EPANETpdd by allowing each emitter to have its own empirical exponent. However, EPANETpdd fails due to convergence-related issues when applied to medium and larger or more complex WDNs.

Liu et al. (2011); Siew and Tanyimboh (2012); and Jun and Guoping (2013) introduced backtracking, line search, and relaxation of parameter techniques to correct the nodal heads only (i.e., not flows) in EPANET. Alternatively, Giustolisi et al. (2011) proposed an entirely new system tool, WDNetXL, for simulating WDN under both DDM and PDM conditions. However, all these approaches exhibit one or more of the following limitations for wider implementation: (1) they require underlying algorithm modifications to be made, (2) they are not in the public domain, (3) they are iterative in nature, (4) they have been demonstrated on limited case studies often involving small or simple networks, and (5) they are unable to handle EPS analysis.

A second principal way of simulating pressure-deficient conditions in a pipe network is by using DDM methods that add artificial hydraulic elements to the network nodes (e.g., valves, emitters, reservoirs, and pipes) without the need to introduce a NHFR function. This is followed by a DDA run in an iterative manner until convergence is achieved. Ozger and Mays (2003); Ang and Jowitt (2006); Todini (2006); and Suribabu and Neelakantan (2011) connected (and removed, when necessary) artificial reservoirs to pressure-deficient nodes to calculate the actual flows delivered. However, this type of approach has the drawback of withholding demands until the minimum nodal pressure head is satisfied, which usually needs a large number of iterations to converge. Furthermore, it can be very difficult to apply this type of approach to large networks, because adding and removing artificial reservoirs at various stages of the iterative methodology is not an easy task, especially under EPS analysis (Wu 2007; Wu et al. 2009). This is because the network topology must be changed iteratively at each time step to identify the correct pressure-deficient and pressure sufficient nodes. 
Hayuti et al. (2008) found that the number of iterations in the aforementioned approaches could be reduced by using the successive solution-seeking method, but even with this reduction, the overall computational time is still substantial. Also, Jinesh Babu and Mohan (2012); and Sivakumar and Prasad (2015), addressed the limitation of the Ang and Jowitt (2006) method by introducing artificial flow control and check valves to ensure that flows into artificially added reservoirs did not exceed the required demand and to restrict the negative pressure in the network, respectively. This led to a smaller number of iterations when compared to the Ang and Jowitt (2006); and Todini (2006) approaches. Nevertheless, the artificial reservoirs still could not simulate the important partial flow between the minimum and the desired pressure head levels. Gorev and Kodzhespirova (2013) tried to address this shortcoming by introducing an additional artificial pipe with a suitable resistance coefficient at all demand nodes. Although the results were obtained with a single hydraulic run, the approach only supported the specific, parabolic type of NHFR (Wagner et al. 1988).

Sayyed et al. $(2014,2015)$ replaced the artificial reservoir and pipe with a suitably chosen flow emitter to reflect the properties of each node in the network. Both algorithms delivered good results under steady-state analysis. However, neither algorithm considered the effect of minimum pressure head level for a head value higher than zero. In summary, the implementation of existing PDM approaches by introducing artificial elements in all the network nodes results in the increased size and computational time of the pipe network problem. This, in turn, often leads to convergence failures and crashing hydraulic solvers, especially in large WDNs under EPS analysis.

Even though the single-iteration PDM approach (SIPDM) proposed in this thesis in chapter 3 makes use of the similar sequence of artificial elements (check valve-flow control valve-emitter) to model pressure-deficient conditions, unlike in other approaches (Sayyed et al. 2014, 2015), the artificial elements in SIPDM are added on a selective basis, i.e., only to pressure-deficient nodes with demands (i.e., demand nodes with pressure head less the required value: $P_{n, \text { avl }}<$ $P_{n, \text { req) }}$. These nodes are identified by running the DDM-type hydraulic solver (e.g., EPANET) once before PDM simulation. This is very important, especially in the case of larger, real-life networks, where it is well known that typically only a small part on the network (i.e., a small number of nodes) is likely to experience 
pressure-deficient conditions during a failure event (e.g., a pipe burst or equipment failure). Therefore, there is no need to add the proposed artificial elements to all demand nodes as other PDM methods currently suggest. As will be shown in the case studies that follow, this leads to several benefits, including (1) increased computational efficiency of hydraulic calculations; (2) more stable convergence of the PDM simulator, ending in more realistic results; and (3) less effort required to add artificial elements to the network, which, in this case, can be done manually. However, this is virtually impossible with other approaches. Furthermore, the approach works well under both steady-state and EPS conditions, even in the case of large and complex WDNs, as illustrated in chapter 4.

\subsubsection{Real-time WDS models}

Real-time WDS model is defined as an integration of network hydraulic and quality model with operations data collected and stored via SCADA. Real-time water system models (e.g., hydraulic and quality) have become an important tool in planning and design of WDSs, and are now more frequently used in operations under a wide range of demand loading and operating conditions (Boulos et al. 2014; Savic 2014; Sunela 2017). Mass and conservation equations, and reaction kinetics are used in these models to predict pressure, flow and water quality (i.e., movement and transformation) for specified system properties and operating conditions. These models are capable of providing powerful tools for determining responses to different operational (i.e., various demand stress conditions) and management strategies in order to meet required performance goals (Boulos et al. 2014).

In order to achieve these goals, the models require an accurate, continuously updated view of WDS status condition. This can accomplished by synthesizing SCADA and other real-time telemetry data with networks models.

The resulting networks models provide utility operators continuous real-time insights regarding water network performance. A constant stream of data for small time steps (i.e., 15, 30, $60 \mathrm{~min}$ ) coupled with predictive modelling capabilities, enables network operators to quickly assess events as they occur, 
identify potential problems before they reach critical level, respond to operational challenges, and minimize potential impacts (Walski 2015; Rasekh 2015).

For example, network operators can analyse the effect of predicted low storage tank level on network hydraulics and determine consumers who will be likely adversely affected by low pressure and service interruptions (Kang 2014). Alternative operating scenarios can be quickly analysed and compared to find the most appropriate solution. Operators can also assess the effects of main breaks and other equipment failures, and maintain or repair; as well as any other planned or unplanned incidents (Paluszczyszyn 2015; Odan et al. 2015). These models can also predict import pressure and flow key network parameters, where data loggers are not available and predict systems performance should SCADA feeds go offline. Introducing real-time modelling to networks can help network operators to change network mode from fully reactive to proactive network management (Boulos et al. 2014; Savic et al. 2014). All above can then beneficially results more efficient and low-cost operations systems, greater network integrity and ultimately improve network maintenance and customer service.

One area that has not received much attention is the use of real-time models in emergency planning and response (e.g., response to pipe burst impacts). However, simulation models have been used for emergency planning and response and there is a significant potential for additional application in support of decisions makers (Walski 2015). WDS operators rely heavily on their SCADA systems for information during emergencies. However, SCADA systems cannot be used to analyse the impacts of emergency response (Boulos et al. 2014). By combining the live information from a SCADA system with the power of a hydraulic model to interpolate and extrapolate system behaviour, decision makers can obtain a much better picture of measures to deal with emergencies.

There are some preconditions for using a real-time model in emergencies. To be useful in an emergency response situation, the model must be calibrated for the area of interest and an individual who can run the model must be available. The model user must also have information on the current state of the system. Initial and boundary conditions that were used in building the model may not be appropriate for the given situation. The model user must be able to quickly or automatically open/close valves, turn pumps on/off, adjust demands, set tank levels and other intervention settings in the model (Alonso et al. 2010; Rasekh et 
al. 2014). There are several situations where a model can be helpful in decision support for example detecting an emergency (e.g., pipe burst) through detection system, simulating no-action impact (i.e., impact assessment), testing alternatives (e.g., interventions), and restoring normal operation (i.e., pre-failure event conditions).

The above review reveals the importance of using real-time models for various WDS purposes. For example, real-time hydraulic model can be used to assist network operator to evaluate the impact of a failure event such as pipe burst on the future status of the system assuming 'do nothing' scenario. The same model can be used to evaluate the impact of alternative interventions under failing network conditions, especially when a quick decision needs to be taken. If this model is coupled with an optimisation model then the network interventions can be optimised too resulting in more consistent/objective operational interventions being suggested to the network operator. Such a methodology is currently lacking in the literature.

\subsection{Real-time Operational Optimization Models of WDS}

These models were extend the use of WDSs to assist network operators improve efficiency and ensure more reliable operation (e.g., increasing benefits) at minimum cost level under normal and abnormal network operation conations. A typical complete real-time operational optimization model framework comprises SCADA connection, demand forecasting, optimization algorithm, and solution evaluation module (Sunela 2017). The models automatically read real-time field data, instantly update the network model, and determine for example pump, and valves operation schedules that will produce minimum operating cost that satisfying the network required performance such as boundary limits for flows, pressures and velocities, total pump flow capacity and tank trajectory curves.

Linking optimum mass-balance storage model with the real-time network model is common in WDS applications which used to produce optimal solutions quickly for improving system operating performance in the short-term horizon, typically for the next 24 hours. The linking models spontaneously defines the massbalance storage model, consider the variation in demand, control elements (e.g., pumps, valves) and other network factors in a timely manner (i.e., each time step 
in the simulating period). Optimization methods are then used to optimise operational interventions (such as pump operations). The generated optimal solutions can be then send to the integrated SCADA system and network operators for implementation purposes.

In general, optimisation methods in WDS modelling can be divided into three categories: deterministic, stochastic and mixed techniques. The deterministic techniques include enumeration, linear programming (e.g., optimal power surface (OPUS) methodology), nonlinear programming (e.g., Mock Tree II algorithm), and dynamic programming. The stochastic techniques consist of metaheuristics population-based algorithms (e.g., genetic algorithms, evolutionary strategies, harmony search, particle swarm optimization, ant colony optimization, shuffled leaping frog algorithm, etc.) and metaheuristics single point-based methods (e.g., simulated annealing, Tabu search, simple $(1+1)$ evolutionary strategies, trajectory or local search methods, etc.) (Maier et al. 2015; Sunela 2017). Mixed techniques combine both deterministic and stochastic methods. Some examples include combining linear programming and GA (Reis and Pinheiro 2005), linear programming with hybrid discrete dynamically dimensioned search (HD-DDS) (Puleo et al. 2014), particle-swarm with harmony search (Geem 2009), and linear programming with greedy algorithm (Giacomello et al. 2013). The above optimization methods are frequently used in WDS optimization for the purpose of network operation (e.g., pump scheduling, pressure management problems, risk analysis) as well as calibration (e.g., calibration of pipes internal roughness, leaks detection), and (re)design problems (e.g., optimum pipe sizes, network structure, valve location).

In comparison to deterministic, stochastic methods are efficient, both in terms of precision and computational effort, in solving many real-life WDS problems (Maier et al. 2015; Sunela 2017) in near real-time. Stochastic methods not required to formulate the problem in analytical form and the formulation can be nondifferentiable. Also, the advantages include their ability to handle discrete choices and to identify near-optimal solutions to complex optimisation problems (Simpson et al. 1994). Finally, they are capable of identifying a set of promising solutions that may be compared on other non-quantifiable criteria and are suited to discrete problems (Simpson et al. 1994). 
Genetic Algorithms (GAs) are probably the most well-established class of metaheuristics used for solving WDS problems and are inspired by various mechanisms of biological evolution (e.g., reproduction, mutation, crossover, selection, etc.) (Nicklow et al. 2010). The genetic algorithm starts with a randomly generated population of solutions, and after each iteration, the best solutions are most likely to survive into the next iteration (generation). The surviving solutions exchange design variable values (genes) with each other, and there is a chance for mutations (random changes in design variable values).

However, the computational efficiency of using the meta-heuristic optimization approach involving GAs at identifying the real or near real-time global or nearglobal optimum solutions is still a challenging problem, due to two reason: (i) great number of hydraulic simulations are required, even though a single simulation can be reasonably fast, the simulations can still be the bottleneck in the optimization process; (ii) some of the performance metrics are computationally demanding, especially in large WDSs. Consequently, limitations in available computational resources could have a negative impact on the quality of the evaluation of optimization algorithm performance. The magnitude of this problem could be reduced, but not completely resolved, by using: (i) surrogate modelling (e.g., ANNs), (ii) parallelization (i.e., by utilizing multiple CPU cores or cloud computing services), (iii) population pre-processing (e.g., fixed-length encoding, such as Dynamic Parameter Encoding (Schraudolph and Belew 1992)) and (iv) heuristic information to reduce the size of the search space, such as graph decomposition methods Kadu et al. (2008); Zheng et al. (2011, 2014); global sensitivity analysis (Fu et al., 2011) or engineering judgement and historical information (Pasha and Lansey, 2010). Having said that, a reduction in the size of the search space generally may results in approximation, either because a number of decision variables have to be fixed prior to optimisation or because the nature of interactions among the decision variables precludes effective size reduction. This could potentially exclude the region that contains the true global optimum.

The above reveals that solving optimization problems and obtaining optimal operational interventions in near real-time is still a difficult task that has not been resolved completely, i.e. in a satisfactory manner so far. Therefore, in the work presented in this thesis, a novel method for the optimal selection of interventions 
that is based on two new effective preparation stages (i.e., offline and online) is developed with the aim to reduce the search space and speed up the follow on optimisation process. This, in turn, enables real response problems to be solved in near real-time (see chapter 3 ).

\subsection{Summary}

This literature review highlights the need for continued evolution and innovation in the field of real-time modelling specifically operational response methods for WDSs in the UK (and worldwide) to improve the current best practice for the management of emergency conditions. A number of existing real-time modelling, impacts assessment and response methods for handling failure events and mitigating/ reducing their impacts are discussed and the need for further comparative studies of the various methods and metrics is highlighted, including additional testing on complex real-world case studies. The review reveals the pertinent timing of this work for the water industry and derives numerous areas for further qualitative and quantitative research.

Based on the literature review, this thesis aims to develop a near real-time response methodology to reduce negative impacts of unplanned events (specifically pipe bursts) in a WDS in a more automated way and by optimising the network interventions in near real-time. The interventions considered in the thesis are based on the current practice of the UK water industry. The aim is to provide additional support for WDS operators when making relevant decisions by mitigating adverse impacts and prioritizing timely interventions in a more effective way, especially in cases when failures are more complex in nature (e.g., affecting a larger part of the water distribution system and consumers) and decisions need to be made quickly. This, in turn, is expected to result in improved operational efficiency and emergency preparedness, reduced water loss and shortened response time. Finally, the new methodology should enable less experienced operators to make equally good decisions, all with the aim to improve customer service and protect the WDS. The complete framework and detail of the near real-time response methodology developed in this thesis are presented in the following chapter (chapter 3). 


\section{CHAPTER 3: METHODOLOGY FOR REAL-TIME OPERATIONAL RESPONSE TO FAILURE EVENTS IN A WDS}

\subsection{Introduction}

Water distribution systems are regularly subject to operational failure conditions caused either by emergencies or planned maintenance events. Under such circumstances, certain elements and parts become inoperable, and the system can no longer provide the minimum level of service to consumers. Dealing with failure conditions in a WDS and providing continues services is one of the primary functions of control room operators in the UK water utilities. The process of discovering that a WDS is not functioning normally, investigating potential incidents (pre-event stage) and deciding on how to deal with them (post-event stage) is still challenging, even with recent progress in monitoring and communication technologies. The immediate initiation of effective response actions can be critical and essential for reducing and limit spreading the adverse impacts (i.e., supply interruption, pressure drops) resulting from failure events. However, so far, most decisions and response tools are currently made on an adhoc basis, primarily based on the experience of skilled operators.

The developed real-time operational response methodology presented in this chapter of the thesis aims to reduce adverse impacts under unplanned event in a specific WDS (e.g., pipe burst) in a more automated/optimal way and in near real-time by using locally available network interventions. The methodology is based on the current practice in the UK water industry. It is intended to provide additional support to WDS operators when making relevant decisions by mitigating adverse impacts and prioritizing timely interventions in a more efficient way, especially in cases when failures are more complex in nature (e.g., affecting a significant part of the water distribution system and consumers), and decisions need to be made quickly. This, in turn, is expected to result in improved operational efficiency and emergency preparedness, reduced water loss and shortened response time. Finally, the methodology enables less experienced operators to make equally good decisions, all with the aim to improve customer service and protect the WDS. 
This chapter is organized as follows. After this introduction, a general overview of the real-time response methodology and its phases following on from a failure event is presented in section 3.2. Once this is done, section 3.3 provides the methodological details of the segmentation method (i.e., first response phase) that enables to isolate an event location and to identify the interrupted consumers from the water source(s). The recovery phase is then formulated and presented in section 3.4 with all the required calculation details and solution method explained. An impact assessment model able to capture various operational aspects of adverse impacts caused by a failure event is described in section 3.5. Finally, in section 3.6, the entire chapter is summarized.

\subsection{Response Methodology Overview}

The automated response methodology, presented here, involves two basic phases (as shown in Figure 3.1). In the first phase, following a failure event, the affected part of the network is isolated from the rest of the system by closing appropriate valves. The affected part is kept isolated until the failed pipe undergoes repair or is fully replaced. The isolation aims to eliminate the impacts of the failure event spreading to the entire WDS, for instance pressure drops. The isolation leads to interrupted service to consumers that were supplied from the now disconnected segment and may likely affect additional consumers downstream of the isolated area. The affected consumers can be classified to: (1) those that are unaffected, (2) those that have completely lost the water supply and (3) those that are partially affected, i.e., experience a drop in pressure and, if the failure event persists long enough to drain tanks, may lose service (Walski 2015). Once isolation is completed, further decisions should be taken so that the operation of the "non-isolated" parts of the network experience minimal negative impacts (e.g., low pressure, supply interruptions) and the effects of failure event are contained. This can be performed through second phase, i.e., recovery, which involves selecting and implementing an optimal combination of operational interventions for network operators as temporary alternatives, i.e., until pre-event failure conditions are restored. 


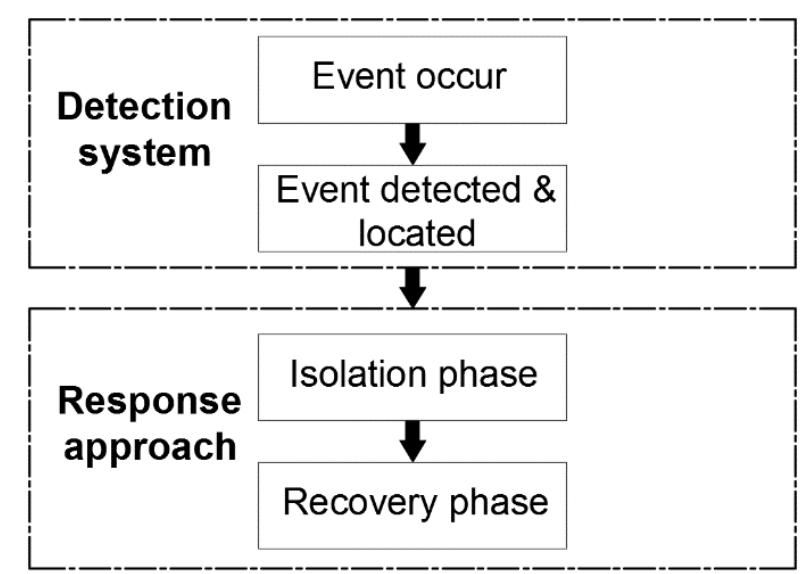

Figure 3.1. Phases of the response methodology.

For the particular event in the WDS that is being detected at time $t_{d}$, located at time $t_{f}$ and isolated at time $t_{i}$, the impact (IM), defined here as the cumulative undelivered volume of water to consumers over a specific impact horizon $T_{h}$, is estimated next in a near real-time operation mode. The impact (i.e., $I M_{R}$ ) of doing nothing is assessed first, shortly after event detection and location, with the aim to assess the scale of the event impact, i.e., to see if something needs to be done. This is achieved by conducting the necessary online hydraulic simulation analysis that makes use of the SCADA data (e.g., observed tanks levels, flows, pressures). This data are normally collected in a constant cycle of pre-specified time steps (e.g., every 15 minutes). The impact horizon is measured over a prespecified time frame (e.g., 6, 12, or 24 hours) from the moment of locating the event failure $\left(t_{t}\right)$. Once it is concluded that something should be done, a number of optimal intervention solutions are identified and introduced to aid temporary recovery until the failure element is fully replaced or repaired. These solutions are automatically selected from a pre-defined interventions list (for a specific WDS) by using a suitable methodology (please see section 3.4.4). The SCADA data are used in the process to assess the effect of introducing different interventions (impact reduction) and also to continuously monitor the affected area. 


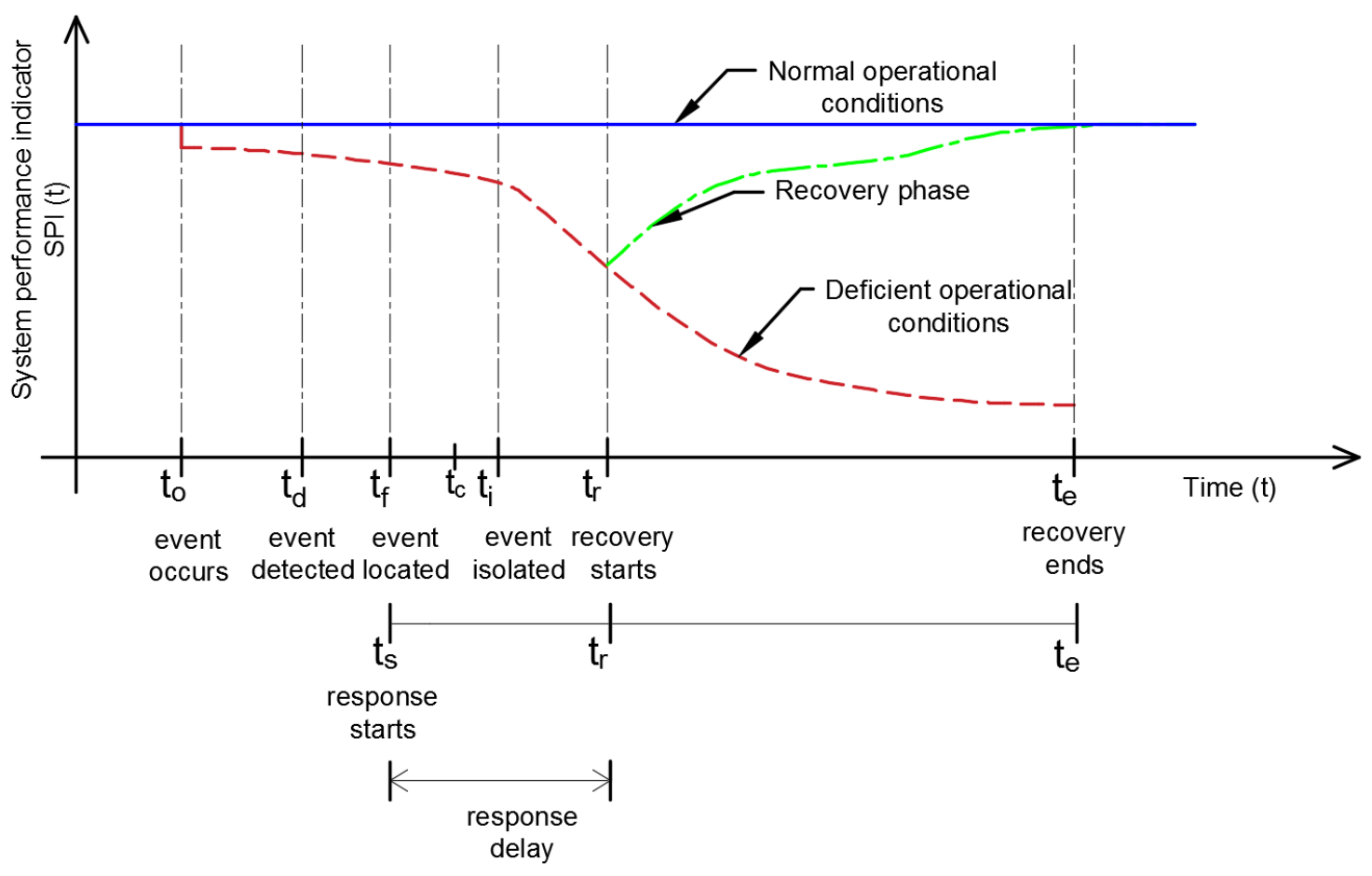

Figure 3.2. Failure event development stages vs system performance.

Figure 3.2 provides an illustration of the development stages of a failure event against system performance indicator (SPI) in real-time. The selection of the suitable SPI relies upon on the particular service provided by the WDS under analysis as explained in section 2.3.1. In this work, the SPI is assumed to be the ratio of water delivered by the system to the water required by the consumers (over the time). The SPI, therefore, has a value between 0 and 1 , where 0 indicates that no water is delivered to the system and 1 is the target SPI value (i.e., under normal operation conditions). Note that other, more complex and refined impact assessment models could be developed (Mansoor 2007; Bicik et al. 2010) or company specific models could be used. The point is that the response methodology shown here is generic in nature and, as such, it can accommodate any of these models as long as they can quantify the impact of operational interventions considered in terms of specific SPI(s) used.

Note further that the diagrammatic process presented in Figure 3.2 is shown only for illustration purposes and is not drawn to scale. The solid blue line indicates system performance under normal operational conditions. At the time to the system experiences a failure event (such as a pipe burst) at a specific location in 
the network. The system performance may suddenly deteriorate (as illustrated by the dashed red line), with this process continuing until the event is detected at time $t_{d}$ and then located at time $t_{t}$. At that time $\left(t_{t}\right)$, the first phase of the response methodology is applied and the event is isolated at time $t_{i}$. Still, the system performance is likely to deteriorate further due to pressure deficient conditions resulting from isolating a part of the network (including the failure event location) and this may reach a minimum at time $t_{e}$.

In order to alleviate the impact of system deterioration from time $t_{f}$ to $t_{e}$, a recovery phase is introduced. Optimal recovery interventions may be introduced in the field at any time from $t_{r}$ to $t_{e}$. Recovery phase calculations can be initiated immediately after identifying shut-off valves to isolate the failure event. This involves generating a list of initial, possible interventions, followed by the selection of the optimal ones by trading off the cost of interventions and likely negative impact reduction. The details of the process for the screening and obtaining optimal recovery interventions is given in section 3.4.4.

From time $t r$, the system performance (indicated by dashed green line) starts recovering to the normal operating conditions, as illustrated in Figure 3.2. The figure also shows the response delay as the time period between when the location of the event is identified and when the recovery intervention solutions are implemented.

\subsection{Isolation Phase}

Once the early warning system has detected a failure event(s) in the WDS, it is crucial to take appropriate, timely decisions to isolate the affected part of the network. This is done by identifying an appropriate set of shut-off valves, which need to be closed in order to separate (i.e., isolate) the affected network segment(s) from the rest of the WDS. Isolation should result in the minimum impact (i.e., supply interruptions) on consumers through locating the nearest set of isolation valves that need to be closed. The isolation phase enables network operators to retrieve all necessary information about the location of valves and to identify consumers on segment (s) that are affected by a total loss of supply service. 
The affected network segment(s) is/are divided into two types: (i) Regular network segment(s) that involve(s) the isolated part of the network that contains a failed element (e.g., failed pipe), and (ii) Unintended network segment(s), defined as part of the network that is unintentionally disconnected from the water sources, as a consequence of isolating the regular segment(s). A segment(s), including both regular and unintended, are likely to involve additional network elements (e.g., pipes, nodes, other network equipment) that are not damaged, including the affected consumers who are isolated in the process. Depending on the location and the number of valves that need to be closed, the number of elements in a segment may vary. For instance, a single segment isolation needs to have two valves at both upstream and downstream ends of the failed network element (e.g., pipe), which is unlikely to exist in a real WDS.

Isolation process calculations and implementation starts when the location of the failure event has been confirmed by the detection system. The primary purpose of separating the failure event is to prevent both economic and social impacts associated with it and this may involve the following:

- Lost water: the quantity of water lost until the affected element(s) isolated after the failure event has occurred can be considerably large especially if a large pipe fails.

- Interrupted supply: the failure event may likely cause certain changes in hydraulic behaviour (e.g., flow, pressure) of the WDS. This changes may include, for example, dropping the pressure head in the system to an insufficient level (i.e., dropped below required standard level). In addition to this, the supply can get disconnected at a number of connection points (e.g., nodes), and this, in turn, may lead to leaving a number of consumers without water.

- Structural damages: the surrounding infrastructure can be affected by failure event. However, this depends on the type of event failure and its scale. For example, a large burst can cause flooding of streets and houses.

- Other potential impacts: for example direct and indirect costs that could affect water utilities resulting from lost water, property damage, lost revenue due undelivered demand, and third party damage; the 
introduction of pollutants causing deterioration of the water quality; traffic congestion, closing of businesses, environmental impacts, etc.

The impacts of failure events listed above can be both expensive and harmful to consumers, utilities and environment, therefore, are undesirable. The overall damage resulting by a failure event depends on the time when the event occurred and the time when it isolated. To minimise the relevant impacts associated with failure event a real-time technique for quick detection and location is necessary, especially in case of pipe burst and leakage events. The duration of time for which the network segments remain isolated is defined as a time between the point when the maintenance team obtains access to valves that should be closed to the point when the failed system element (e.g., a burst pipe) is either replaced or repaired. The duration of the repair depends on the availability of resources (e.g., material, tools, and personnel).

Once an alarm has been raised and a potential failure event located, the isolation phase derives and extracts in real-time the list of isolated elements and set of shut-off valves from the network information. This information retrieves automatically from network input data file, e.g., by reading and automatically processing the relevant data from the EPANET2.0 input file. The information contains necessary detail such as size, location, and settings for each network element (e.g., valve, pipe). The detail also includes the number of consumers allocated to each network node. The network input information is updated periodically to consider the modifications on valves and other equipment (e.g., pipes added or removed, inoperable valves, etc.). The lists are presented to the network operators for making suitable decisions such as sending maintenance team to close valves (e.g., in case if the valves are not remotely controlled) and notify the isolated consumers.

The identification of the list of isolated network elements (e.g., pipes, nodes) belonging to both regular and unintentional segments (in addition to the calculation for locating the set of nearest isolation valves) is carried out by using new network segmentation method which is presented in the following section. 


\subsubsection{Segment identification methodology}

The identification of network segment(s) that should be isolated following a failure event is based on the information contained in two network topological matrices. These matrices are the Node-Link matrix (defined here as $A_{n l}$ ) and the ValveLocation matrix (defined here as $A_{v}$ ). The form of both matrices is similar, with each column corresponding to a specific network node (e.g., reservoir, tank, demand node) and each row corresponding to a particular network link (e.g., pipe, pump). All elements in these two matrices have a value of either 1 or 0 . The matrix element $i j$ has a value of 1 if the link in row $i$ (i.e., $l_{i}$ ) is connected to the node in column $j$ (i.e., $n_{i}$ ) of the $A_{n l}$, and it has a value of 0 otherwise. On the other hand, each element of matrix $A_{v l}$ gets the value of 1 if a valve placed on a link $l_{i}$ and next to a node $n_{i}\left(\right.$ i.e., $\left.V\left(l, n_{j}\right)=1\right)$, and gets the value of 0 otherwise.
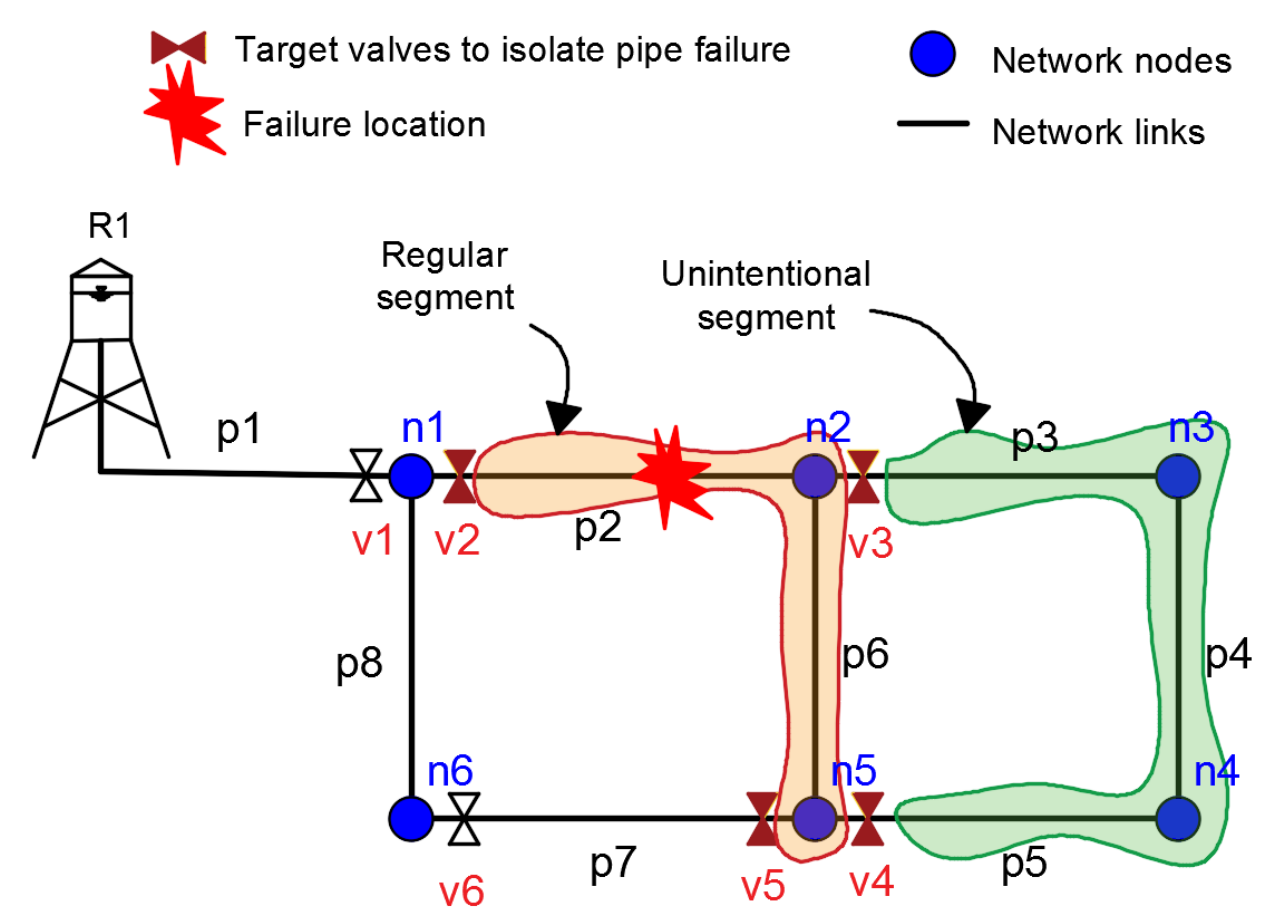

Figure 3.3. Regular and unintended network segments under $\mathrm{p} 2$ failure for a simple network.

The regular network segments are identified through the following steps:

1. Construct valve deficiency matrix $A_{v d}=A_{n l}-A_{v l}$. 
2. Construct matrix $V_{p}$, which is obtained by removing zero columns from matrix $A_{v l}$ and then splitting columns having more than one element with value greater than zero into multiple columns in a way to have only one element with value greater than zero in each column.

3. Construct symmetric matrix $V=\left(A_{v d} / V_{p}\right) \times\left(A_{v d} / V_{p}\right)^{T} ; V(I, j)>1=1$.

4. Pipes belonging to each segment can be identified by reading non-zero elements (i.e., pipe index) in each column of matrix $V^{m}$. Where $m$ is the maximum allowed pipes belong segment.

5. For each pipe, belonging to each regular segment in step 4, read non-zero element value for the corresponding row number for the pipe from matrix $V_{v d}$ and then read the corresponding column number (i.e., node) to the segment.

6. Identify the list of isolation valves belong each segment from matrix $A_{v v \text {. }}$

The unintended network segments are identified through the following steps:

1. For each regular segment, construct new $A_{n l}$ (i.e., $A_{n l}^{s i}$, where $i$ indicates regular segment order) matrix by removing pipes and nodes belong target regular segment $i$.

2. Apply steps 3,4 , and 5 considering $V_{s i}=\left(A_{n l}^{s i}\right) \times\left(A_{n l}^{s i}\right)^{T}$.

3. Segments that do not contain water source nodes are considered as unintentional segments for the target regular segment i.

To illustrate the implementation steps of the above network segmentation method and detail of the proposed matrices, the proposed algorithm was applied to a simple network shown in Figure 3.3 under a hypothetical pipe failure scenario, i.e., burst on pipe p2. The network consists of: one water source (i.e., R1), hence $n_{s}=1$, where $n_{s}$ is the total number of source nodes in the network; six demand nodes, $n_{j}=6$, where $n_{j}$ is the total number of junctions in the network (i.e., demand 
nodes, dummy nodes, hydrant points); eight links, $n_{l}=8$, where $n_{l}$ is the total number of pipes and pumps in the network; and, finally, five isolation valves, $n_{v}=$ 5 , where $n_{v}$ is the total number of isolation valves in the network. Thus, matrices $A_{n /}$ and $A_{v l}$ are as follows:

Table 3.1. Node-Link and Valve-Location matrices for the network in Figure 3.3.

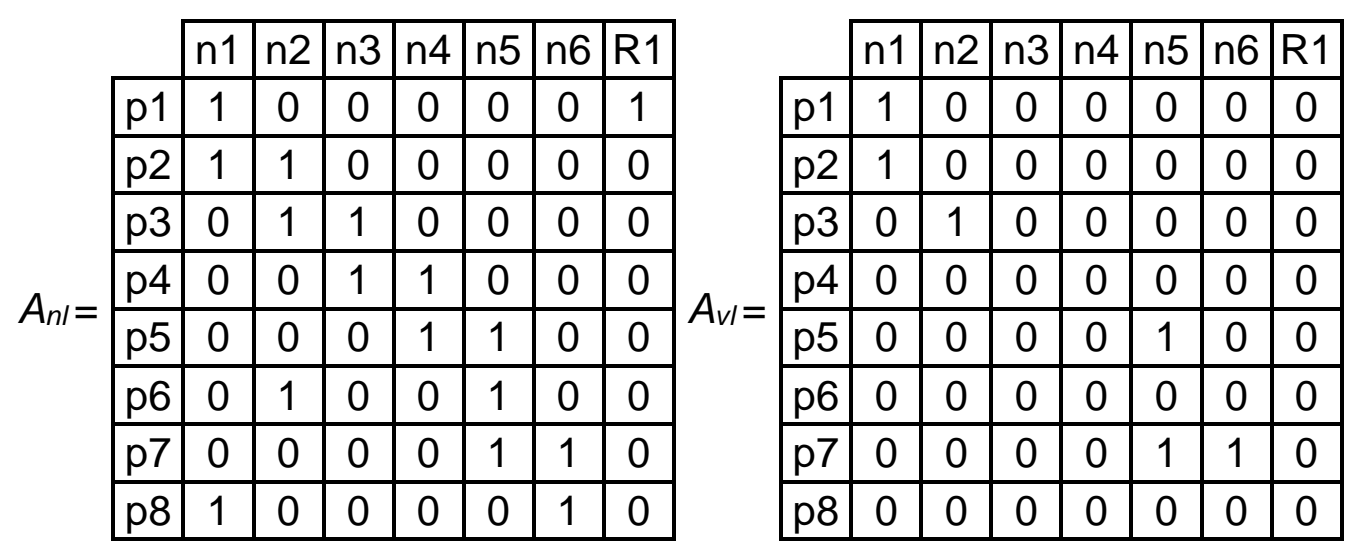

From the $A_{n l}$ and $A_{v l}$, the valve deficiency matrix $\left(A_{v d}\right)$ is obtained by subtracting $A_{v l}$ from $A_{n l}$ (i.e., $A_{v d}=A_{n l}-A_{v l)}$. A "1" in the $A_{v d}$ matrix means no valve is placed at that position. Because no valve is placed at the position, pipe failure cannot be confined at that position and it propagates to the adjacent pipes and nodes. The $A_{v d}$ matrix enables us to trace the valveless paths. The pipes, nodes, and valves located on these paths together form a segment. Accordingly, the structure of $A_{v d}$ for our simple example can be written as follows (Table 3.2): 
Table 3.2. Deficiency $\left(A_{v d}\right)$ and $V_{p}$ matrices for the network in Figure 3.3.

$A_{v d} \quad$\begin{tabular}{|c|c|c|c|c|c|c|c|}
\cline { 2 - 7 } & $\mathrm{n} 1$ & $\mathrm{n} 2$ & $\mathrm{n} 3$ & $\mathrm{n} 4$ & $\mathrm{n} 5$ & $\mathrm{n} 6$ & $\mathrm{R} 1$ \\
\hline $\mathrm{p} 1$ & 0 & 0 & 0 & 0 & 0 & 0 & 1 \\
\hline $\mathrm{p} 2$ & 0 & 1 & 0 & 0 & 0 & 0 & 0 \\
\hline $\mathrm{p} 3$ & 0 & 0 & 1 & 0 & 0 & 0 & 0 \\
\hline $\mathrm{p} 4$ & 0 & 0 & 1 & 1 & 0 & 0 & 0 \\
\hline $\mathrm{p} 5$ & 0 & 0 & 0 & 1 & 0 & 0 & 0 \\
\hline $\mathrm{p} 6$ & 0 & 1 & 0 & 0 & 1 & 0 & 0 \\
\hline $\mathrm{p} 7$ & 0 & 0 & 0 & 0 & 0 & 0 & 0 \\
\hline $\mathrm{p} 8$ & 1 & 0 & 0 & 0 & 0 & 1 & 0 \\
\hline
\end{tabular}$V_{p}=$

\begin{tabular}{|l|l|l|l|l|l|l|}
\hline $\mathrm{p} 1$ & 1 & 0 & 0 & 0 & 0 & 0 \\
\hline $\mathrm{p} 2$ & 0 & 1 & 0 & 0 & 0 & 0 \\
\hline $\mathrm{p} 3$ & 0 & 0 & 1 & 0 & 0 & 0 \\
\hline $\mathrm{p} 4$ & 0 & 0 & 0 & 0 & 0 & 0 \\
\hline $\mathrm{p} 5$ & 0 & 0 & 0 & 1 & 0 & 0 \\
\hline $\mathrm{p} 6$ & 0 & 0 & 0 & 0 & 0 & 0 \\
\hline $\mathrm{p} 7$ & 0 & 0 & 0 & 0 & 1 & 1 \\
\hline $\mathrm{p} 8$ & 0 & 0 & 0 & 0 & 0 & 0 \\
\hline
\end{tabular}

Once the above three matrices (e.g., $A_{l n}, A_{v l}, A_{v d}$ ) are established, the segment identification method is used to automatically identify the elements (e.g., nodes, pipes) within the regular network segment, which should be isolated to contain the failure element location (e.g., p2 in our simple network example). This can be done starting from the symmetric matrix $V$, which can be calculated as follows:

$$
V=\left(A_{v d} / V_{p}\right) \times\left(A_{v d} / V_{p}\right)^{T}
$$

The matrix $V_{p}$ is obtained by removing the columns of zeros in $V_{v l}$ and dividing the remaining columns that have more than one non-zero element into multiple columns in order to get only one non-zero element in each column, as shown in Table 3.2.

It is fair to say that the non-zero element $V(i, j)$ of $V$ in each column or row (considering $I \neq j$ ) provide the $i$-th indices of the links which are adjacent to $j$-th link. For instance, the adjacent link to link p3 is p4, as shown in Figure 3.3 and Table 3.2. Accordingly, the non-zero elements in each column or row of $V^{2}$ represent the links which are connected by a path of length (number of links on the path) less than or equal to two (Brualdi and Ryser 1991). By taking into account the power $m$ for $V$, it is possible to state that the non-zero elements in each column or row of $V^{m}$ (after replacing non-zero elements in $V^{m}$ by one) represent the links that are connected by a path (i.e., network segment) of length less than or equal to $m$. Note that for a fully connected network the matrix $V^{m}$ 
becomes full (i.e., it will not have zero elements), $m$ depends on the maximum and the minimum path lengths between two pipes of the network, i.e., maximum or minimum number of links that are connected by one path (Brualdi and Ryser 1991).

For our simple network, Table 3.3 shows the matrix $V^{m}$ (with $m=4$ ), indicating those links in Figure 3.3 that have non-zero values in the same unique columns (e.g., pipes that are connected by one path). For example, columns two and six show that p2 and p6 belong to a single segment (i.e., s2), as shown in Table 3.4 (the details for other segments are reported in the same table). It is also interesting to note that for the $N$ valve rule scenario (i.e., having an isolation valve at each end of all pipes in the network), $V^{m}$ is an identity matrix (or each pipe will be belong a segment and the number of regular segments will equal to the number pipes in the network). By replacing non-zero elements with the corresponding link number in the column's header, by removing duplicate columns and zero elements, regular network segment lists for all links in the network are obtained. It can be observed from Table 3.3 that the proposed network segmentation method can generate simultaneously all possible segments for all links in the network. This is important in case of having two or more coinciding failure events at the different parts of a particular network.

Table 3.3. Matrix $V$ and $V^{m}$ (with $m=4$ ) for the network in the Figure 3.3 .

\begin{tabular}{|c|c|c|c|c|c|c|c|c|}
\cline { 2 - 9 } \multicolumn{1}{l|}{} & $\mathrm{p} 1$ & $\mathrm{p} 2$ & $\mathrm{p} 3$ & $\mathrm{p} 4$ & $\mathrm{p} 5$ & $\mathrm{p} 6$ & $\mathrm{p} 7$ & $\mathrm{p} 8$ \\
\hline $\mathrm{p} 1$ & 1 & 0 & 0 & 0 & 0 & 0 & 0 & 0 \\
\hline $\mathrm{p} 2$ & 0 & 1 & 0 & 0 & 0 & 1 & 0 & 0 \\
\hline $\mathrm{p} 3$ & 0 & 0 & 1 & 1 & 0 & 0 & 0 & 0 \\
\hline $\mathrm{p} 4$ & 0 & 0 & 1 & 1 & 1 & 0 & 0 & 0 \\
\hline $\mathrm{p} 5$ & 0 & 0 & 0 & 1 & 1 & 0 & 0 & 0 \\
\hline $\mathrm{p} 6$ & 0 & 1 & 0 & 0 & 0 & 1 & 0 & 0 \\
\hline $\mathrm{p} 7$ & 0 & 0 & 0 & 0 & 0 & 0 & 1 & 0 \\
\hline $\mathrm{p} 8$ & 0 & 0 & 0 & 0 & 0 & 0 & 0 & 1 \\
\hline
\end{tabular}
\begin{tabular}{l|l|l|l|l|l|l|l|l|l|l|l|l|l}
\hline p1 & p2 & p3 & p4 & p5 & p6 & p7 & p8 \\
\hline
\end{tabular}

\begin{tabular}{|l|l|l|l|l|l|l|l|l|}
\hline $\mathrm{p} 1$ & 1 & 0 & 0 & 0 & 0 & 0 & 0 & 0 \\
\hline $\mathrm{p} 2$ & 0 & 1 & 0 & 0 & 0 & 1 & 0 & 0 \\
\hline $\mathrm{p} 3$ & 0 & 0 & 1 & 1 & 1 & 0 & 0 & 0 \\
\hline $\mathrm{p} 4$ & 0 & 0 & 1 & 1 & 1 & 0 & 0 & 0 \\
\hline $\mathrm{p} 5$ & 0 & 0 & 1 & 1 & 1 & 0 & 0 & 0 \\
\hline $\mathrm{p} 6$ & 0 & 1 & 0 & 0 & 0 & 1 & 0 & 0 \\
\hline $\mathrm{p} 7$ & 0 & 0 & 0 & 0 & 0 & 0 & 1 & 0 \\
\hline $\mathrm{p} 8$ & 0 & 0 & 0 & 0 & 0 & 0 & 0 & 1 \\
\hline
\end{tabular}

Once the links belonging to each segment are identified, the nodes belonging to each segment can be obtained from the matrix $V_{v d}$. This is done by considering for each $i$-th row (i.e., link), in the target regular network segment, the $j$-th non- 
zero elements (i.e., node). For example, nodes $\mathrm{n} 2$ and $\mathrm{n} 5$ belong to the regular network segment s2, as shown in matrix $A_{v d}$ and Table 3.4. Also, it can be seen from the same table that each node only belongs to one single segment.

Table 3.4. Segments isolation detail for the network in the Figure 3.3.

\begin{tabular}{l|l|l|l|l}
\hline $\begin{array}{l}\text { Regular } \\
\text { network } \\
\text { segment }\end{array}$ & Pipe list & Node list & Valve list & $\begin{array}{l}\text { Unintentional network } \\
\text { segment }\end{array}$ \\
\hline s1 & $p 1$ & $R 1$ & $v 1$ & $\begin{array}{l}n 1, n 2, n 3, n 4, n 5, n 6, p 2, p 3, p 4, p 5, \\
p 6, p 7, p 8\end{array}$ \\
s2 & $p 2, p 6$ & $n 2, n 5$ & $v 2, v 3, v 4, v 5$ & $n 3, n 4, p 3, p 4, p 5$ \\
s3 & $p 3, p 4, p 5$ & $n 3, n 4$ & $v 3, v 4$ & - \\
s4 & $p 7$ & - & $v 5, v 6$ & - \\
s5 & $p 8$ & $n 1, n 6$ & $v 1, v 2, v 6$ & $n 2, n 3, n 4, n 5, p 2, p 3, p 4, p 5, p 6, p 7$ \\
\hline
\end{tabular}

The next step is to associate each segment to the corresponding valve subset (the set of nearest isolation valves to isolate each network segment). This subset can be obtained in two stages from the $A_{v /}$ matrix. In the first stage for every link in the regular network segment, each element (e.g., valve $V\left(l, n_{j}\right)$ ) in the corresponding $i$-th row in matrix Avi is examined. If a " 1 " is found, then that valve is added to the valve subset list. In the second stage, for each node in the regular network segment, each element (e.g., valve $V\left(l i, n_{j}\right)$ ) in the corresponding $j$-th

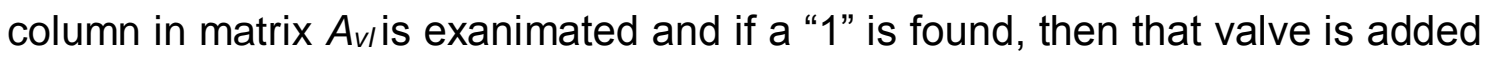
to the valve subset list.

For example, Figure 3.3 highlights all isolation valves that should be closed to separate segment $s 2$ from the rest of the network due to pipe p2 failure. For segment $s 2$, we have the links $\{\mathrm{p} 2, \mathrm{p} 6\}$ and nodes $\{\mathrm{n} 2, \mathrm{n} 5\}$. Thus, the value ("1" or "0") in all elements in row two and six is checked firstly. Accordingly, the valve $V\left(l_{2}, n_{1}\right)$, i.e., v2, located in the second row is only added to the valve subset list at the first stage. In the second stage elements $V\left(I_{3}, n_{2}\right)$,i.e., v3, in column two (assigned column for node $\mathrm{n} 2)$, and $V\left(p_{5}, n_{5}\right)$,i.e., $v 4$, and $V\left(p_{7}, n_{6}\right)$, i.e., v5, in column six (assigned column for node n6) are added to valve subset list as shown 
in Table 3.4 (also the valve subset list for the rest network segments are given in the same table).

The final step is to calculate unintentional network segment. As mentioned earlier, due to isolating a regular segment which contained failure element, other parts of the network may unintentionally disconnected from the water sources in the network. For instance, due to the isolation of $s 2$, the nodes n3, n4 and pipes p3, p4, p5 do not have a path from the water source R1. As the results, the consumers allocated within these elements will not receive any supply, and they must be counted as consumers affected by the pipe p2 failure.

The calculation for identifying unintended network segment(s) for each regular network segment is started by constructing a modified Node-Link matrix $\left(A_{n l}^{s i}\right)$, where I refers to the order of regular segment $s$, from the original Node-Link matrix $\left(A_{n 1}\right)$. This can be done by removing all columns assigned to nodes and all rows assigned to links in the regular network segment from the $A_{n l}$. For example under isolating segment $\mathrm{s} 2$ scenario, the structure of $A_{n l}$ (i.e., $A_{n l}^{s 2}$ ) will be as shown in Table 3.5, after deleting row two and six, and column two and five.

Next, a modified $V$ matrix for $A_{n l}^{s i}$ is constructed using the following equation:

$$
V_{s i}=\left(A_{n l}^{s i}\right) \times\left(A_{n l}^{s i}\right)^{T}
$$

Considering the power $m$ for $V_{s i}$, all link segment (group of links which are connected together by one segment, i.e., path) can be obtained for the modified topology network matrix $A_{n l}^{s 2}$ by considering non-zero elements in each column or row (Bruadli and Ryser 1991). For instance for $m=4$, the detail for these link segments is given in Table 3.5 (under removing s2 scenario). 
Table 3.5. Node-Link and $V^{m}$ matrices for the network in Figure 3.3 after removing segment $\mathrm{s} 2$.

\begin{tabular}{|c|c|c|c|c|c|c|c|c|}
\cline { 2 - 8 } \multicolumn{1}{l|}{} & $\mathrm{n} 1$ & $\mathrm{n} 2$ & $\mathrm{n} 3$ & $\mathrm{n} 4$ & $\mathrm{n} 5$ & $\mathrm{n} 6$ & $\mathrm{R} 1$ \\
\hline \multirow{4}{*}{$A_{n l}^{s 2}$} & $\mathrm{n} 1$ & 1 & 0 & 0 & 0 & 0 & 0 & 1 \\
\hline $\mathrm{p} 3$ & 0 & 0 & 1 & 0 & 0 & 0 & 0 \\
\hline $\mathrm{p} 4$ & 0 & 0 & 1 & 1 & 0 & 0 & 0 \\
\hline $\mathrm{p} 5$ & 0 & 0 & 0 & 1 & 0 & 0 & 0 \\
\hline $\mathrm{p} 7$ & 0 & 0 & 0 & 0 & 0 & 1 & 0 \\
\hline $\mathrm{p} 8$ & 1 & 0 & 0 & 0 & 0 & 1 & 0 \\
\hline
\end{tabular}

\begin{tabular}{|c|c|c|c|c|c|c|}
\hline & $\mathrm{p} 1$ & p3 & $\overline{\mathrm{p} 4}$ & p5 & p7 & p8 \\
\hline \multirow{6}{*}{$\frac{p}{p}$} & 1 & 0 & 0 & 0 & 1 & 1 \\
\hline & 0 & 1 & 1 & 1 & 0 & 0 \\
\hline & 0 & 1 & 1 & 1 & 0 & 0 \\
\hline & \begin{tabular}{|l|}
0 \\
\end{tabular} & 1 & 1 & 1 & 0 & 0 \\
\hline & 1 & 0 & 0 & 0 & 1 & 1 \\
\hline & 1 & 0 & 0 & 0 & 1 & 1 \\
\hline
\end{tabular}

Further, it can be observed from $V^{m}$ matrix that $\mathrm{p} 1, \mathrm{p} 7$, and $\mathrm{p} 8$ are connected by one link segment (e.g., su1), whilst p3, p4 and p5 are belong to another link segment (e.g., su2). Next the list of node belong each of these link segments are identified by considering for each $i$-th row (link) the $j$-th non-zero elements (node) in matrix $A_{n l}^{s_{i}}$ (e.g., $A_{n l}^{S_{2}}$ under segment s2 failure scenario). Hence, R1, n1, and n6 are belong to the link segment su1, and $\mathrm{n} 3$ and $\mathrm{n} 4$ are belong to the link segment su2. Therefore, the link segments that not include any source nodes are considered as unintentional network segment. For example, the only water source for our simple network shown in Figure 3.3 is R1and it is not include in su2, which means that this link segment is considered as unintentional network segment under s2 failure.

\subsection{Recovery Phase}

When the location of failure event is identified, the isolation phase begins immediately, followed quickly by the beginnings of recovery phase. Recovery phase is aimed at alleviating the negative impacts of failure (e.g., supply interruptions, pressure drops) to consumers located in the area adjacent to the affected isolated area. This can be performed by selecting a combination of suitable operational recovery interventions from a number of choices available in a specific WDS. The detail of recovery phase is provided in the following subsections. 


\subsubsection{Recovery interventions}

The recovery interventions are used as temporary preventative actions and operate on the system to reduce negative impacts of a failure. The recovery interventions considered in this work include the following (assuming a WDS comprised of a number of DMAs): (a) opening/closing boundary valves to bring water into the affected part of the network from within the DMA and/or from nearby DMA(s), (b) modifying PRV outlet pressure setting(s), where possible, to aid recovery by increasing the pressure, and (c) installing temporary overland (pipe) bypasses between two suitable fire hydrant points with the aim to bring water into and increase pressure in the affected part of the network. These interventions are used regularly in the engineering practice in the UK. Of course, other interventions also exist (e.g., supply of water to the affected area by trucks or bottles), but are not considered here as the focus of this work is on network type interventions (please see section 2.2.4). Also, the operational interventions enlisted above are considered here because they could generally be applied to different companies and systems. Note also that the interventions considered cover a range of possible interventions, from less costly and efficient (e.g., changing PRV settings or manipulating valves) to more expensive and able to bring water into the affected area (e.g., overland bypasses) and hence provide sufficient complexity to illustrate and validate the proposed response methodology. Finally, the response methodology presented here is generic in nature, hence additional response operations could be added to it in a straightforward manner.

Various types of valves are available in a WDS. This work investigates the effect of isolation valves and PRVs in reducing impacts in the recovery phase. Isolation valves are commonly utilized in WDS to separate a pipe segment for repair or to divert flow in a pipe segment. The PRV is used to reduce the pressure of the water conveyed through it, to obtain a regulated and constant pressure value at its outlet, as well as to meet the water demand of consumers at adequate pressure. The pressure at a PRV exit is set to a pre-specified service level and during recovery, it can be increased by a certain amount/percentage.

The fire hydrants are ordinarily implemented as stand-post or underground hydrants, approximately at a distance of $100 \mathrm{~m}$ from each other, to withdraw contaminated water and/or for firefighting purpose in the system. Flexible hose 
pipes (e.g., lay flat hose) can be used to create overland bypasses, and they can be modelled as dummy pipes (with initially closed status) in the system. The lengths of the overland bypasses are calculated based on the distance between two target hydrants while their diameters are selected based on the available nominal sizes in the system (please refer to section 3.4.4 for further details). In general, overland bypass pipes could be placed along roads to avoid traffic jams and other obstacles.

The selection of a suitable intervention(s) from the above options should be performed based on the criteria such as negative impact reduction, operational cost, time to implement and potential unintended consequences resulting from implementing the intervention (as explained in section 2.2.5). Performance in preventing or reducing the negative impact of the failure on consumers is the most important criterion in this work. A recovery intervention should be efficient and reliable. For instance, installing bypass is a promising tool for delivering water, but a lack of understanding of a given WDS hydraulics behaviour could lead to inefficient or worsened consequences (e.g., flow direction reversal, discoloration problems). Also, interventions that come with lower costs for equipment and installation are more preferable.

On the other hand, and from a technical perspective, the number of implemented interventions should be as small as possible as this tends to reduce the associated operational cost. It also makes the implementation simpler, hence more reliable. The performance of each recovery intervention (or a mix of recovery interventions) in terms of impact reduction needs to be evaluated. This requires simulating the consequence of these interventions by using a WDS hydraulic solver (or otherwise). Ultimately, selecting the best mix of interventions to implement requires using optimisation. This is likely to require a long computational time, i.e., the time that may not be available when solving the response problem in near real-time. Hence, it is necessary to narrow down the list of recovery interventions and then select the optimal mix of these. This can be done by applying the automated technique described in the section 3.4.4. 


\subsubsection{Interventions selection criteria}

Two criteria are used here to quantify the performance of operational recovery interventions, once the failure event is located and isolated. The first criterion measures the negative impact of an event on consumers (IM). The second criterion measures the operational recovery effort (as cost) required to fully or partially recover from the failure, i.e., to return the network to pre-failure condition. A developed impact assessment model is used to measure negative impact IM over the short term horizon $T_{h}$ (e.g., 6, 12, or $24 \mathrm{hr}$ ). The impacts are evaluated from the perspective of consumers' requirements (e.g., supply, pressure) rather than of the water utility. The detail of impact assessment model and calculation for estimating impact $I M$ are given in section 3.5.

The operational effort required to reduce the negative impact of a failure event can be surrogated with the total number of interventions associated with their costs and it is calculate as follow:

$$
C I N V=\sum_{i=1}^{n i v} w_{\downarrow} I V_{i, T_{h}}+\sum_{j=1}^{n o b} w_{o} O V_{j, T_{h}}+\sum_{k=1}^{n p r} w_{p} P R V_{k, T_{h}}
$$

Where:

$C I N V=$ total operational cost for the recovery interventions

$I V, O V$ and $P R V=$ candidate isolation valves, overland bypasses and pressure reducing valves, respectively, whose operation mode may change during recovery intervention phase over horizon $T_{h}$

niv, nob and $n p r=$ total number of feasible isolation valves, overland bypass pipes and PRVs in the network for a specific failure event in a specific WDS

$i, j$ and $k=$ the index for isolation valves, overland bypass pipes and PRVs $w_{v}=$ unit cost weight for changing the status of an isolation valve in the network (surrogate for actual unit cost)

$W_{0}=$ unit cost weight for installing an overland bypass pipe in the network $w_{p}=$ unit cost weight for changing the setting of a PRV in the network. 
$I V_{i, T_{h}}$ has a value of 1 if the operational status (close/open) of the $i$-th valve changed during recovery phase (i.e., over time horizon $T_{h}$ ) from the current status (open/close) and zero otherwise. $O V_{j, T_{h}}$ has a value of 1 if $j$-th overland bypass is used (i.e., 'opened') and zero if it is not used (remains closed) during recovery phase (i.e., over time horizon $T_{h}$ ). $P R V_{k, T_{h}}$ gets a value of one if the pressure at the $k$-th $P R V$ outlet is equal to the current pre-specified value and a value of integer larger than one otherwise.

The unit cost (i.e., $w_{p} w_{o}, w_{v}$ ) values can be provided by the local water utilities and they can determined based on size, location, and operational costs. It is worth to mention that the unit cost of changing the operation mode of an isolation valve or PRV (i.e., $w_{p}, w_{v}$ ) it may small when compared to installing an overland bypass between two hydrants (i.e., $w_{0}$ ).

The reference impact value $I M R$ is calculated assuming a "do nothing" intervention. This reference value can be used for assessing the relative effect of possible recovery interventions.

\subsubsection{Intervention optimization problem}

The selection of recovery interventions is driven by the minimisation of $I M$ (volume of undelivered water to consumers) and minimisation of CINV (number of operational recovery interventions). The mathematical formulation of the twoobjective optimization problem is as follows:

$$
\operatorname{Min} \quad f(X)=(I M, C I N V)^{T}
$$

Where:

$$
X=\left(x_{i}^{I V}, x_{j}^{O V}, x_{k}^{P R V}\right) \quad i=1, \ldots \ldots, n i v ; j=1, \ldots \ldots, n o b ; k=1, \ldots \ldots, n p r
$$

$X$ is the vector of problem decision variables representing different interventions; $x_{i}^{I V}$ represents the status of valve $i$ and it takes value of a $\{0\}$ for closed status and $\{1\}$ for opened status; $x_{j}^{O V}$ represents the status of overland bypass $j$ and it 
takes a value of $\{0\}$ for closed status and $\{1\}$ for opened status; $x_{k}^{P R V}$ is the pressure setting at the exit of $P R V k$ and it takes one of the following integer values $\left\{1,2,3,4, \ldots, p r_{s}\right\}$, where $p r_{s}$ is the total number of settings for $k P R V$ candidate. Each integer represents a predefined increased percentage in the pressure setting at the exit of the PRV (s), according to a given mapping, for example: $1=$ no increase, $2=$ increase by $5 \%, 3=$ increase by $10 \%$ and $4=$ increase by $15 \%$.

The optimization constraints are as follows:

$$
\begin{aligned}
& x_{i}^{I V} \in\{0,1\} \\
& x_{j}^{O V} \in\{0,1\} \\
& x_{k}^{P R V} \in\left\{1,2,3,4, \ldots . p r_{s}\right\} \\
& I M \leq I M_{R} \\
& I N V \leq I N V_{\max } \\
& \sum_{l \in \text { in }(n)} Q_{l}(t)-\sum_{l \in \text { out }(n)} Q_{l}(t)-Q_{n, \text { del }}(t)=0 \quad, n \in N \\
& K_{l} \cdot Q_{l}^{\alpha}(t)=P_{u}(t)-P_{d}(t) \quad, \text { for } I=(u, d) \in L \\
& P_{n, \text { min }}(t) \leq P_{n, \text { avl }}(t) \quad, n \in N
\end{aligned}
$$

Where $I N V_{\max }$ is the maximum allowed number of interventions; $Q_{I}(t)$ is flow rate in link $I=(u, d)$ at time $t ; \operatorname{in}(n)$ and $\operatorname{out}(n)$ represent the set of pipes that are supplying to and delivering flow from node $n$ at time $t$, respectively; $L$ is the number of network links; $K$ / is the head friction loss coefficient at link $I ; \alpha$ is HazenWilliams coefficient $(\alpha=1.852)$; and $P_{u}(t)$ and $P_{d}(t)$ are the head pressures at the ends of pipe / at time $t$, respectively.

Constraint (3.9) ensures that the introduced recovery interventions improves the operational conditions. Constraints (3.11) and (3.12) represent mass 
conservation and energy conservation equations for the network. Finally, constraint (3.13) ensures that the available pressure at each network node is above the minimum required.

\subsubsection{Intervention selection methodology}

The solution method starts with preparing the list of potential operational recovery interventions. This list is obtained by narrowing down the number of interventions available in a specific WDS through two preparation stages (i.e., offline and online) as shown in Figure 3.4. The calculation for the preparation stage starts after the location of the failure event was identified at time $t_{f}$ followed by identifying the set of isolation valves to isolate event. The initial list of possible interventions is generated from network elements (e.g., isolation valves, fire hydrants, PRVs) data in the first stage (i.e., offline) without estimating the impact IM. In the second stage, the list obtained in the first stage is further screened based on real-time hydraulic analysis results for the network (e.g., IM and the direction of flow) over horizon $T_{h}$ (assuming the event is isolated at $t_{i}$ ). The key advantage of the above procedure is a decreased search space size and hence reduced computational time required to search for optimum recovery solutions. The successful intervention candidates from the two preparation stages are then optimised at the final, optimization stage to find the optimal mix of recovery interventions which can be identified at time $t_{c}$.

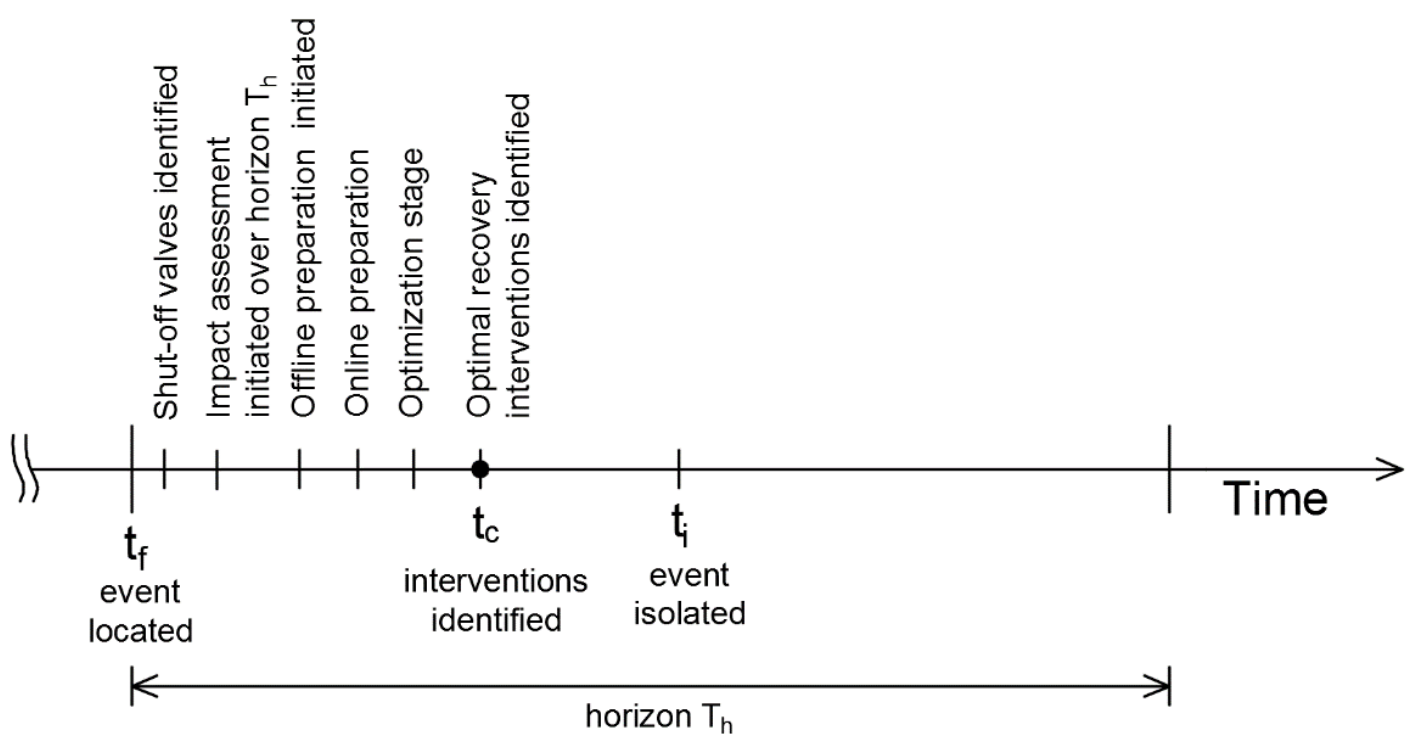

Figure 3.4. Stages for identifying optimum interventions in the recovery phase. 


\subsubsection{Offline preparation}

After identifying shut-off valve list (e.g., isolation phases) and estimating impact assessment over the horizon $T_{h}$, the offline preparion stage is introduced. In this stage the intervention lists (e.g., lists of isolation valves, fire hydrants and PRVs in a given WDS), are prepared from the network data at this stage (e.g., by automatically processing the relevant data from the EPANET2.0 input file by using suitable Matlab script). The data contains necessary information for each network element (e.g., size, location, settings, etc.), please see section 3.4.5. This data is updated periodically to consider the modifications on network elements (e.g., valves, hydrant and pipes added or removed, inoperable valves, etc.). When a specific failure event is detected and located in real-time, the initial list (i.e., obtained offline) of possible operational recovery interventions is developed automatically from the above lists and it involves:

1. All isolation valves (except those used for isolating the failure location) in the DMA, where the failure event is detected and located, and those located on the neighbouring WDS DMA(s), if any. The affected DMA(s) are defined here as the DMA(s) that have deficient-pressure condition in one or more nodal demands. This/these DMA(s) are identified by running the PD hydraulic solver over horizon $T_{h}$.

2. The isolation valve(s), if any, connecting affected $\mathrm{DMA}(\mathrm{s})$ and the nearby unaffected DMA(s).

3. Possible overland bypass routes connecting nearby (pairs of) fire hydrant points in the affected DMA(s). Furthermore, for a specific failure event in the WDS, a list of overland bypasses that originate and end in the affected DMA(s) are automatically created (excluding those that start and end in the isolated area or those that start from the affected DMA and end in nearby unaffected DMA(s)). Connecting fire hydrants between the affected and unaffected DMA(s) is allowed if the distance between the two fire hydrants $L D$ is smaller than some pre-specified threshold $L D_{\max }$ (bearing in mind that installing overland bypass between different DMAs is dependent on the network topological conditions as well, e.g., if an accessible route between two fire hydrant points exists or not). The list of 
the above overland bypasses are obtained by applying the shortest path algorithm technique (Gallo and Pallottino, 1988).

4. Overland bypasses that connect fire hydrant points in the unintentionally isolated segments (i.e., nodes and pipes that are separated from water sources in the network because of the isolated failure area) and nonisolated fire hydrant points.

5. PRV(s) located upstream of the affected area.

\subsubsection{Online preparation}

Online preparation is applied in near-real time prior to conducting the actual optimization of interventions. The list of potential candidate solutions (that are obtained from the offline preparations list) is reduced further as follows:

1. The direction of flow at all isolation valves and network pipes parallel to each overland bypass candidates (that were identified at the offline preparation stage) is observed over the horizon $T_{h}$ (see section 3.2). The overland bypass candidates that connect the affected DMA(s) and nearby unaffected DMA(s) are excluded from this evaluation and are directly nominated to point three in this section. The flow directions are detected by using directed Graph Theory (Duval 1988; Cheng et al. 2009) and the SIPDM hydraulic solver. Based on the observed flow directions, the following cases are possible:

a. If the flow direction in each isolation valve, which connects the affected DMA and nearby unaffected DMA, is pointing toward the affected DMA, that isolation valve is nominated for optimization.

b. The isolation valve and overland bypass candidates that do not fall on the critical flow path line are dropped, and the rest of candidates are nominated for the individual evaluation stage (point three in this section). The critical path lines here are defined as the flow paths that feed deficient nodal demands (including the isolated nodes) for 
the most critical time snapshot in the analysed horizon time $T_{h}$ (usually the peak hour of the day). For example, in Figure 3.5, the decisions (i.e., overland bypasses and isolation valves), which are not located on the highlighted critical path flow line (i.e., pipes) that supply water to the deficient nodes $\mathrm{N} 1$ and $\mathrm{N} 2$, are dropped at this stage. This is because they are unable to enhance the flow and pressure conditions at deteriorated nodal demands. Conversely, resetting or installing them could make pressure conditions worse at the other non-affected part of network.

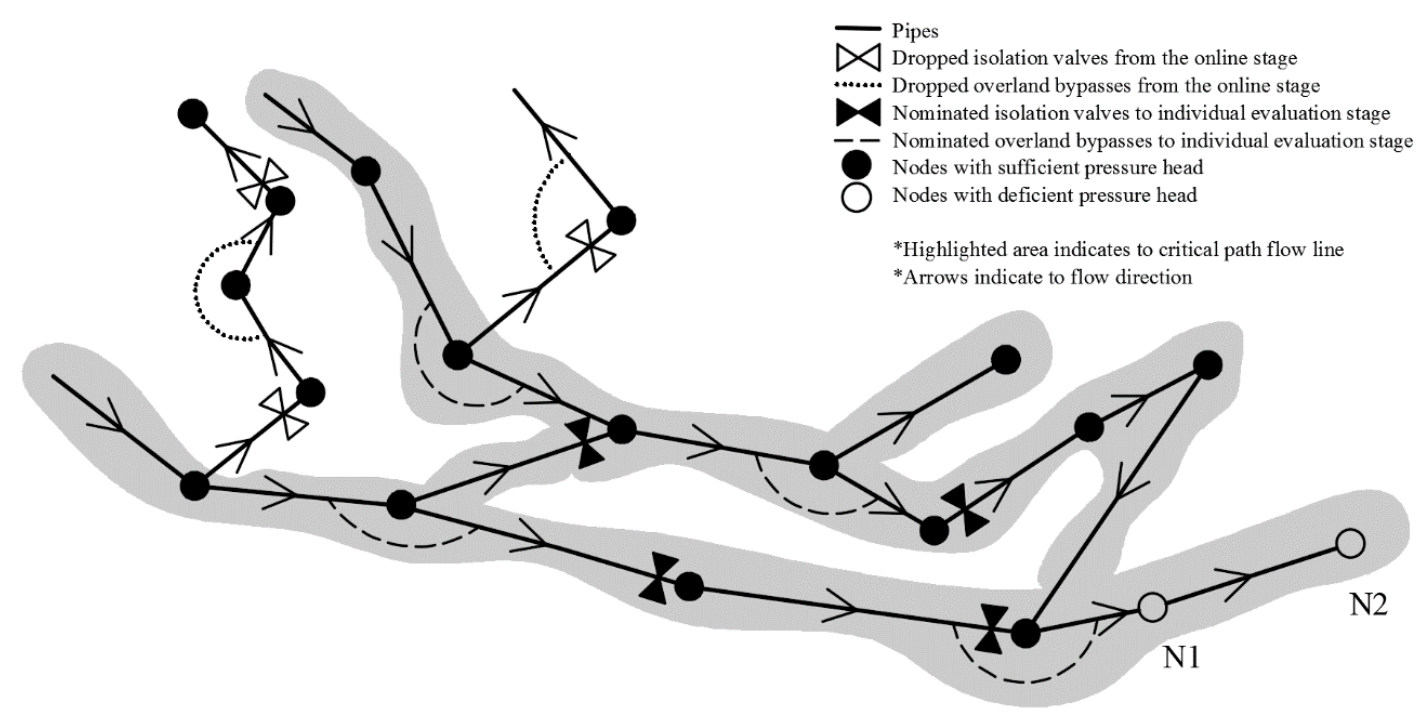

Figure 3.5. Flow path line definition to the deficient nodal demands.

2. Only the PRVs that are located upstream of affected area are nominated for optimization stage. This is done because they can improve pressure conditions downstream.

3. Each isolation valve and overland bypass candidate that remains at the end of point one of online preparation is considered as a potential individual recovery intervention, but only if they produce impact IM less than the impact $I M$ for "doing nothing" intervention scenario (i.e., $I M<$ $\left(M_{R}\right)$. Having said that, if one or more decisions reduce the undelivered volume of water to that which is equivalent to supply the isolated consumers, the recovery phase may stop at this point making 
optimization unnecessary. In that case, the operational recovery intervention with the lowest cost is considered as the optimal recovery solution.

\subsubsection{Optimization of candidate interventions}

At this stage the candidate recovery interventions identified in the online phase are optimised by using objectives shown in equation 3.4 and constraints shown in equation 3.6 through 3.13. This optimisation problem is solved by using the Non-dominated Sorting Genetic Algorithm-II (NSGA-II) (Deb et al. 2002) coupled with the SIPDM hydraulic solver to evaluate solutions. The final set of Pareto optimal solutions obtained could be then presented to the network control room operators to help them choose the intervention(s) to implement in near real-time.

NSGA-II is considered one of the most efficient and popular multi-objective evolutionary algorithms (MOEAs) being used to solve different and complex optimization problems in WDSs (Preis and Ostfeld 2008; Alvisi et al. 2012; Boulos and Wiley 2013). The key advantages of using NSGA-II over other optimization algorithms are reduced computational complexity, speed up of the search process (i.e., evaluation of solutions in near real or real-time), avoidance of repeated solutions, the ability to handle constraints and both real and binary (integer) values (Alfonso et al. 2010; Wang et al. 2013).

The NSGA-II parameter values for the population size, the number of generations, crossover probability and mutation probability are selected based on the recommended settings from the literature (Deb et al. 2002; Reed et al. 2003; Wang et al. 2013). The overall process for estimating optimum decision variables (i.e., recovery interventions) is given in Figure 3.6.

Note that the optimisation-based response methodology is meant to provide only support for the operator who is still in charge of making decisions. The methodology proposed can be helpful, especially when events are more complex, affecting a large area/number of consumers and/or the response needs to be identified quickly. Also, decisions made this way are likely to be rational and consistent. Finally, the methodology should enable less experienced operators to make equally good decisions. 


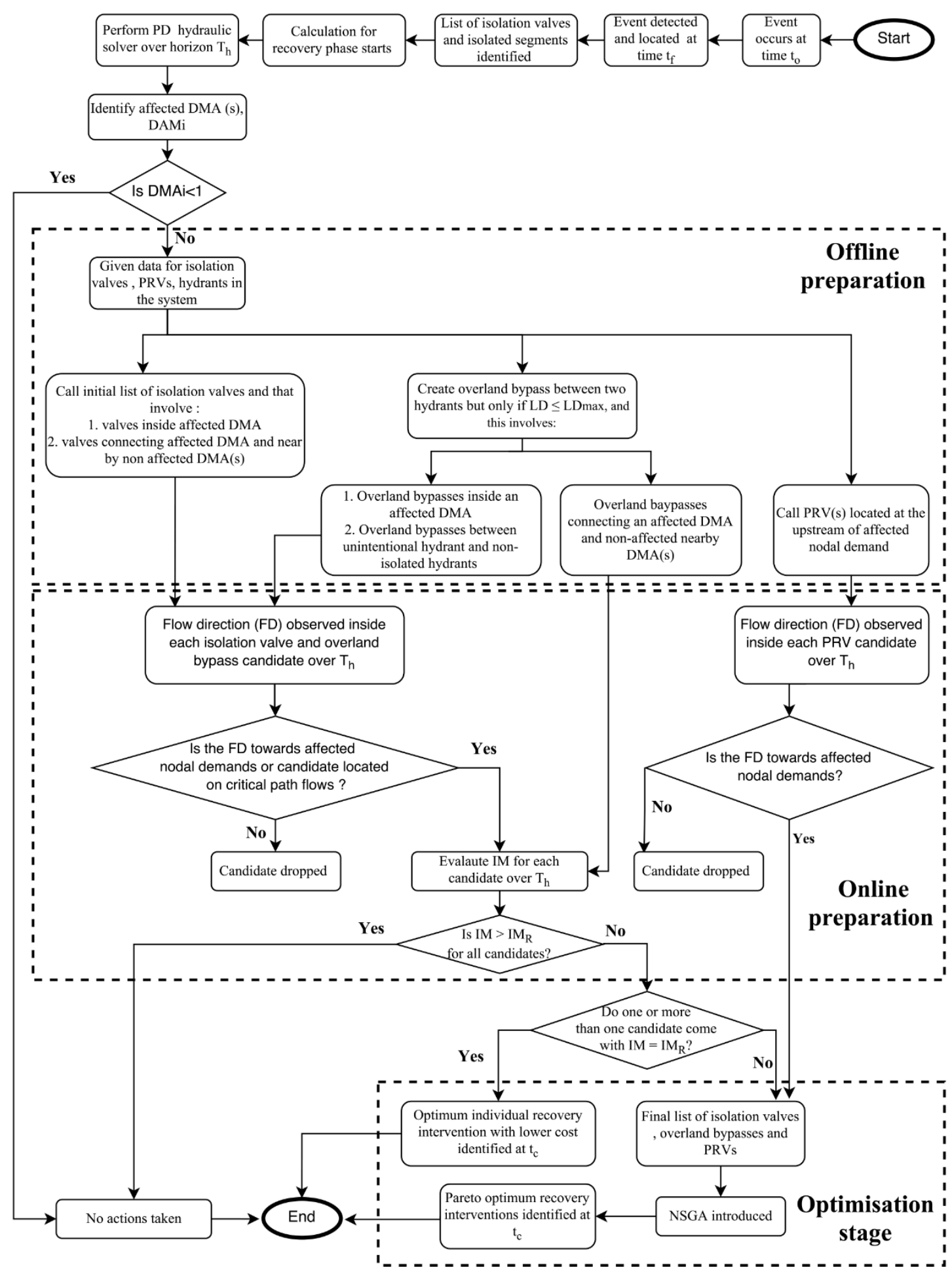

Figure 3.6. The process of estimating decision variables in the proposed response methodology. 


\subsubsection{Simulation of interventions in a hydraulic solver}

The data required for the response methodology (both the isolation and recovery phases) are typically retrieved from the hydraulic simulation solver (e.g., EPANET), or other tools such as Geographic Information System (GIS). These data include the necessary information about network elements such as pipes, control valves, pumps and nodal demands, as well the information about the exact location recovery interventions (e.g., isolation valves, hydrant points, PRVs). Since every hydraulic solver uses different mapping method for introducing elements, above listed information cannot always be extracted. For instance, the well-known hydraulic solver EPANET (which is utilized in the response methodology in this thesis) does not offer a direct and efficient format for an isolating valve or a hydrant. Although, isolation valve can be modelled as the link (valve type) with two nodes and a hydrant point as a node. However, the extra added nodes will increase the overall size of the network, which in turn will slow down the speed of hydraulic solver. This also decreases the performance of the response methodology in identifying optimal recovery solutions in near realtime.

Because of the reason mentioned above, a unique mapping method is used in the proposed response methodology to simulate isolation valves and hydrant points. This is done through developing pre-processor (e.g., Matlab code). The pre-processor allocates every hydrant point that is located at the ends of each feasible overland bypass in offline preparation stage in the network to the nearest existing model node as illustrated in Figure 3.7. Having said that, the length of each overland bypass was measured based on the original location of hydrant points (as mentioned earlier). The overland bypasses with their properties (e.g., length, diameter, roughness) are then added to the network data file as an extra pipe with initially closed status.

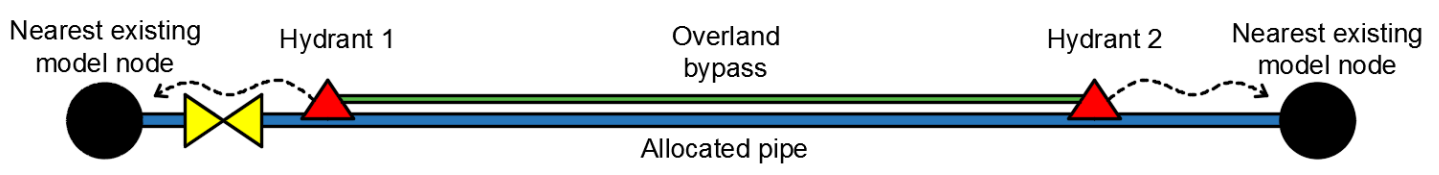

Isolation valve

Figure 3.7. Representation of isolation valve and hydrant in hydraulic simulation solver. 
On the other hand, a pre-processor has allocated each isolation valve in the network to the nearest existing model pipe (i.e., the pipe where the valve is located on it), as shown in Figure 3.7. During hydraulic simulation, the status open/close for each isolation valve is considered by changing the status of the allocated pipe. This assumption may leave the whole section of the pipe out of work when closed, and this, in turn, leads to isolating all consumers within the pipe (e.g., not only the valve location section). However, most hydraulic solvers including EPANET only allocate consumers (e.g., house connections) to model nodes. Finally, each PRV is introduced as a normal link (PRV type), having a node at each end in the network. The setting of each PRV is modified based on pre-defined set of pressure at their downstream (as explained in earlier 3.4.3 section).

\subsection{Impact Assessment Model}

Impact assessment model aims to evaluate and quantify impacts due to failure events as well as to evaluate the recovery interventions efficiency in water distribution system. The model captures the dynamic relationships between the parts of the system and helps to reveal unforeseen influences of failure events in one part of a system on other parts. Such a model enables examination of the linkages between system components, and the changes in the system due to internal or external loading conditions. Impact assessment model can demonstrate the interactions, side effects, and unexpected consequences of actions designed to enhance resilience on a system.

The impact assessment model in this thesis has been developed using a novel impact assessment methodology based on consumers' requirements in terms of pressure head and demand delivered. However, the aspects of water quality are not considered in this thesis. The impact assessment model consists of two components, namely the hydraulic simulation model (i.e., pressure-driven model) and impact measure. The description of each of this component is given in the following sections, 3.5.1 and 3.5.2.

In the hydraulic simulation model, the failure event is represented and the behaviour of network under failure scenario is simulated. The method used to describe the failure events in WDS influences the predictions from the hydraulic 
model. For example, a pipe failure in a WDS can be represented either by just isolating the pipe or by more accurately describing the dynamic failure (immediately after failure) and the isolation (for repair) stages. Besides, hydraulic simulation is needed to model whether a system can supply sufficient quantity and pressure levels for satisfying social functions and consumers requirements.

Impact measure are indicator that describe the behaviour of a system in terms of its operational characteristics. In this thesis, for a water distribution system, performance indicator $I M$ is used to quantify its behaviour mainly based on the nodal outflows, supply pressure head at consumer outlets, and supply interruptions.

\subsubsection{Pressure driven model}

The pressure-driven model (PDM) method developed and presented in this thesis is based on adding a specific sequence of artificial network elements to all pressure deficient nodes with a non-zero demand (i.e., nodes where $Q_{n, \text { req }}>0$ and $\left.P_{n, \text { av }}<P_{n, \text { req }}\right)$. These nodes are identified by running the demand-driven model (DDM) type hydraulic solver (e.g., EPANET) once before PDM simulation. This is very important, especially in the case of larger, real-life networks, where it is well known that typically only a small part on the network (i.e., a small number of nodes) is likely to experience pressure-deficient conditions during a failure event (e.g., a pipe burst). Therefore, there is no need to add the proposed artificial elements to all demand nodes as other PDM methods currently suggest. The sequence of elements added is comprised of a Check Valve (CV), a Flow Control Valve (FCV), an internal dummy node (DN) and an emitter (EM), as shown in Figure 3.8. The role of a check valve is to prevent flow reversal (i.e., inflow at demand nodes when $P_{n, \text { avl }}<P_{n, \min }$ ); the role of the flow control valve is to prevent delivery of a demand larger than required (i.e., limiting the flow in the artificial added elements to $Q_{\text {req) }}$, the internal dummy node is used to link the check and flow control valves in series (an EPANET requirement) and the emitter is used to represent pressure- dependent demand delivery. Given this and the assumed nodal head-flow relationship (NHFR) shown in Equation 3.14, parameter settings of all these elements are allocated as specified in Table 3.6. The proposed PDM solves completely the system of equations that drive a PDM (energy 
conservation, mass balance and NFHR) while also ensures a persistence in time of all the state variables of the network for the EPS. The name SIPDM (Single Iteration Pressure-Driven Method) is used in this thesis to indicate the above PDM method.

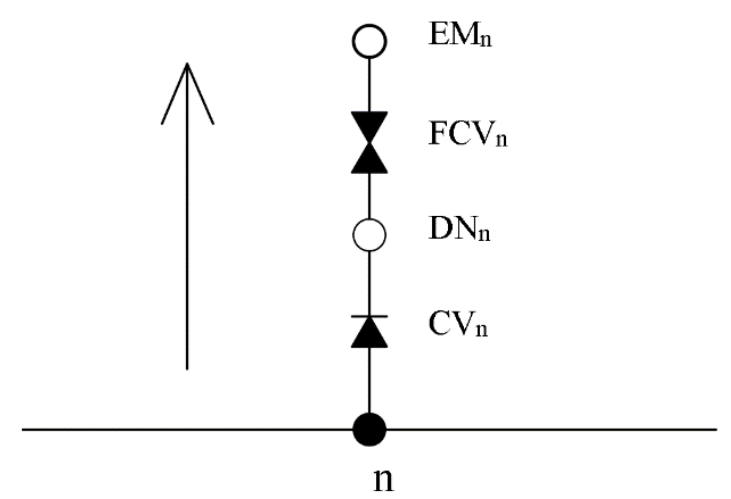

Figure 3.8. Setup at each deficient demand node.

Table 3.6. Properties of added elements to deficient demand nodes.

\begin{tabular}{|l|l|l|}
\hline Network element & Parameter & Setting \\
\hline \multirow{3}{*}{ Demand node $\mathrm{n}$} & $\begin{array}{l}\text { Required } \\
\text { demand }\end{array}$ & $Q_{n, \text { req }}$ \\
\cline { 2 - 3 } & Elevation & ELn \\
\hline \multirow{2}{*}{ Check valve, $\mathrm{CV}_{\mathrm{n}}$} & Length & Small value (e.g., 0.01) \\
\cline { 2 - 3 } & Diameter & large value (e.g., 1000) \\
\cline { 2 - 3 } & Roughness & $\begin{array}{l}\text { Large value (e.g., 140) for Hazen- } \\
\text { Williams formula and small value } \\
(\text { e.g., 0.001) for other formulas }\end{array}$ \\
\hline Dummy node, DN & Elevation & EL \\
\hline Flow control valve, \\
FCV
\end{tabular}


The small length, large diameter and large H-W coefficient values are chosen to ensure that all additional elements do not introduce (significant) head loss between the demand node $j$ and the emitter. The delivered flow $\left(Q_{n, d e l}\right)$ to deficient nodes can now be estimated as follows:

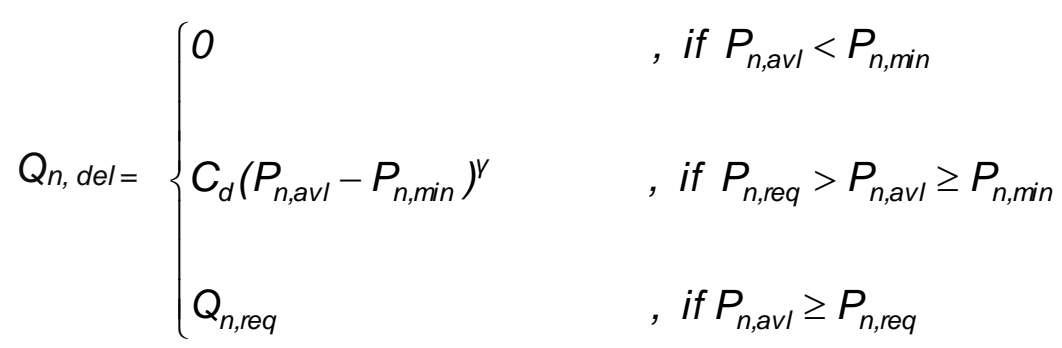

Where $C_{d}$ is the emitter coefficient estimated as $C_{d=} Q_{n, \text { red }} /\left(P_{n, \text { req }}-P_{n, \text { min }}\right)^{v}$ and is the emitter exponent estimated as $y=1 / n_{n}$. The values of both variables depend on the properties of each node that is defined in terms of empirical exponent coefficient $n_{n}$, and the characteristic heads $\left(P_{n, r e q}, P_{n, m i n}\right)$. The overall SIPDM algorithm is presented step by step in Figure 3.9. The procedure shown in Figure 3.9 is for the steady-state analysis in a pressure-deficient network. When performing the EPS analysis, the diurnal demand variation in nodes, the water level in storage tanks and the valve/pump control settings need to be considered over a predefined simulation period. This involves changing the parameters of the connected FCVs and emitters according to the current values of the desired demands in deficient nodes. The number of deficient nodes is estimated by running the DDM solver prior to conducting the SIPDM analysis and it is equal to the number of demand nodes having pressure lower than $P_{\text {req }}$ for the most critical time snapshot in the analysed time period (usually the peak hour of the day). This will guarantee connecting the artificial elements to all potential pressure nodal demands during extended period simulation. Once these nodes are identified, they are able to handle changes from DDM to PDM and vice versa by using the sequence of artificial elements added to these nodes. For example, whenever pressure drops enough for conditions to change from the DDM to PDM, the emitters start 'working' on these nodes. Also, when pressure increases back sufficiently (i.e., above $P_{\text {req }}$ ) and conditions change from PDM to DDM, the FCVs ensure that only the demands required are delivered at these nodes. 


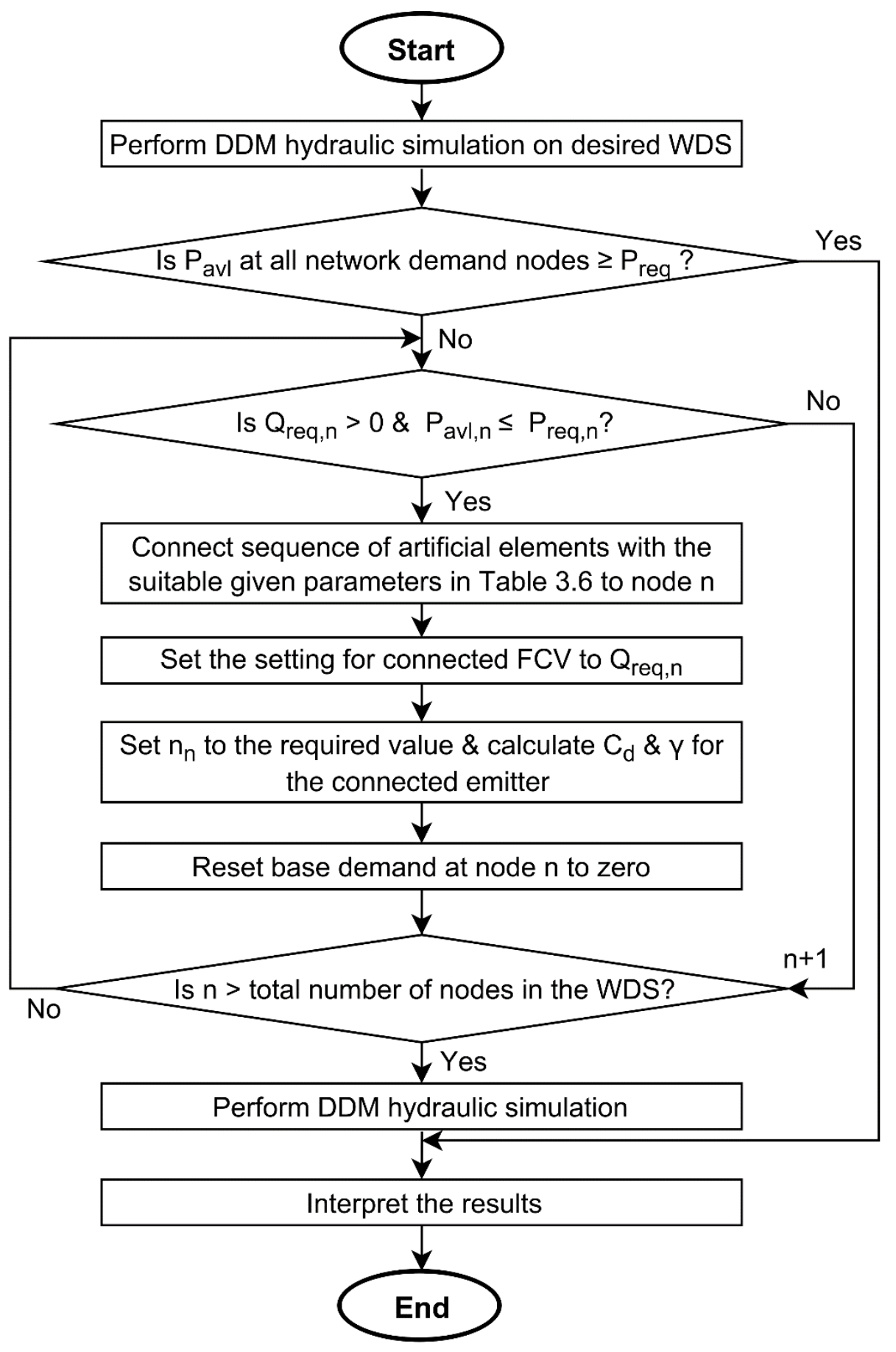

Figure 3.9. Flowchart of the SIPDM approach. 


\subsubsection{Impact assessment}

Impact IM expressing the volume of water undelivered to consumers (including undelivered volume to the isolated consumers) is used here to assess the adverse impacts of low-pressure conditions and interruption from water sources in the network as a result of a failure event over the horizon $T_{h} . I M$ is calculated as system demand shortfall defined as the difference between the total demand required and the total water actually supplied to consumers during the failure event. System demand shortfall is comprised of two components: (1) undelivered demands at nodes that are disconnected either inevitably or unintentionally from the source as a result of isolating the failure event location (2) the amount of water not delivered because of insufficient pressure arising from failure event and its isolation, mathematically impact $I M$ is calculate as follows:

Impact $I M$ is calculated as follow:

$$
M=\left(\sum_{t=t_{t}}^{T_{h}} \sum_{n=1}^{N}\left(Q_{n, r e q}(t)-\left(Q_{n, d e l}(t)\right)\right) \times \frac{\Delta t}{1000}\right.
$$

Where:

$I M=$ impact of failure over the time horizon considered, $\mathrm{m}^{3}$

$Q_{n, \text { req }}(t)=$ required demand at node $n$ and time $t, 1 / \mathrm{s}$

$Q_{n, \text { req }}(t)-Q_{n, \text { del }}(t)=$ undelivered volume of water at node $n$ and time $t$, I

$Q_{n, \text { del }}(t)=$ delivered demand at node $n$ and time $t, 1 / \mathrm{s}$

$t=$ simulation time, hours

$\Delta t=$ simulated hydraulic time step, $\mathrm{s}$

$t_{e}=$ end time of impact horizon, hours

$N=$ number of network nodes

$t=$ moment of detection and location of the failure event

In Equation 3.15, it is assumed that the demands are located at model junctions, and this may introduce a certain level of inaccuracy in the problem being addressed. This is because consumers at each house are connected to a tap along pipe in the real network, not directly to the network node. However, this not considered as critical for assessing and demonstrating the effectiveness of the 
proposed methodology. In cases where this is necessary, the user can always create an EPANET model that models individual house connections which would remove the above inaccuracy. Also, it is well known that nodes that supply critical consumers such as hospitals and schools should be given more priority in providing recovery interventions than other demand points in a specific WDS (Bicik et al. 2009; Rasekh and Brumbelow 2014; Nayak and Turnquist 2016). For simplicity sake, the importance of different consumers was ignored in Equation 3.15 , but could be easily added if required.

The required demands, $Q_{n, \text { req }}(t)$, can be estimated in near real-time over the impact horizon using some demand forecasting model. These models are able to forecast hourly water demands for the next 24-48 hrs (Bakker et al. 2013; Romano and Kapelan 2014; Hutton and Kapelan 2015). Note that, even though not used in this thesis, demand forecasting model can be easily coupled with the real-time recovery methodology shown in this paper.

It is worth mentioning also that when the failure event occurs and the consumers have been alerted, their water consumption behaviour may change (Shafiee and Zechman 2013; Rasekh et. al. 2014; Berglurd 2015). Moreover, consumers may start to suspend using water for drinking, hand washing, bathing and dishwashing purposes. However, uses such as toilet flushing and garden watering may continue (Rasekh et al. 2014). As the hydraulic operating conditions in the system depend mainly on consumer demands, the consumer behaviour thereafter may influence considerably the hydraulic situation in the system. The agent-based models (Shafiee and Zechman 2013; Rasekh et. al. 2014) are usually implemented to incorporate consumers mobility, water demand reduction, and word-of-mouth communication in the response emergency approaches. However, in the response methodology presented here, the effect of consumers demand behaviour (i.e., reduction in required demand $Q_{n, \text { req }}(t)$ ) is not taken into account in the analysis. This was done because it is very difficult to quantify the impact of changed behaviour on the consumption. Also, the planning for the recovery is done assuming more stringent conditions, i.e., the largest possible water consumption in the affected area.

Instead, PDM is used here to try to tackle this shortcoming and to model the reduced water consumption under failure conditions. The predictions of the PDM solver are more realistic and accurate when compared to DDM based solvers 
(Giustolisi et al. 2008; Kovalenko et al. 2014). The PDM hydraulic solver used here is created by adding a specific, artificial set of elements to each deficient demand node in the network and then running the DDM hydraulic solver. The PDM hydraulic solver details can be found in section 3.5.1. Using the PDM solver is an imperfect approximation and the behaviour of consumers under deficient circumstances needs to be studied as part of future research. Having said this, the proposed response methodology is generic in a sense that it can make use of a better corresponding model once it becomes available.

On the other hand PDM solvers represent the most efficient and viable means for predicting the actual demand delivered $\left(Q_{n, \text { del }}(t)\right)$ to network nodes under an array of demand loading (i.e., normal, abnormal) and operating conditions. Where PDM assumed that the actual demand delivered is a function of available pressure at each network nodes (i.e., $P_{n, \text { avl }}(t)$ ) and this function is frequently termed as nodal head-flow relationship (NHFR). The most commonly adopted form of NHFR relationship reads (Wagner et al. 1988), i.e., Equation 2.3, which are used in this thesis and its identical to Equation 3.14, and some alternative formulations can be found in Kovalenko et al. (2014).

In Equation 2.3, water demand $\left(Q_{n, d e l}\right)$ is completely satisfied when nodal pressure head is above a required threshold $\left(P_{n, \text { req }}\right)$. When pressure lies below the minimum acceptable value $\left(P_{n, \min }\right)$, no water is delivered. If the pressure value is between these two characteristic pressure value, water demand is only partially satisfied.

Steady-state and extended period simulations (EPSs) over the horizon $T_{h}$ are used to calculate the system demand shortfall at each network demand node. Steady-state runs are useful for identifying the results of outages that are not particularly long (e.g., less than an hour).With EPS runs, the effects of the failure event (e.g., pipe burst) which take long time to fixed are estimated. EPS runs are much more likely to have nodes that become disconnected such that the hydraulic calculations will not balance. The analyses based on connectivity only and steady-state runs are snapshots that give shortfall in flow units (e.g., liter per second), whereas the EPS runs calculate shortfall in volume units (e.g., cubic meter) as given in Equation 3.15. The detail of the PDM used in this thesis is described in the previous section 3.5.1. 


\subsection{Summary}

This chapter presents a near real-time operational response methodology to reduce the impacts of a failure events (e.g., pipe bursts) in a WDS and restore the flow conditions in the network to the normal service level once the failure location has been located. The methodology proposed is intended to provide support to operators when making relevant decisions, i.e., help them do their job better, especially in cases where failures are complex in nature and decisions need to be made rather quickly, i.e., in near real-time.

Two phases were defined for response methodology: (i) isolation phase, followed by (ii) the recovery phase. The isolation phase identifies the necessary set of isolation valves, which need to be closed to separate the failure event from the rest of network. The recovery involves selecting the optimal combination of individual operational interventions in near real-time, with the aim to minimize both the negative impact of reduced/interrupted supply (resulted because of the isolation) over a predefined impact horizon and the associated operational costs. The optimal set of interventions is determined using a new methodology that makes use of two-objective optimisation coupled with the impact assessment model to evaluate potential solutions and driven by two aforementioned objectives.

The operational interventions considered are opening valves within the affected DMA and between the affected and nearby DMAs, resetting pressure at the outlet of PRV(s) and installing temporary overland bypasses between suitable hydrant points. The impact assessment model is based on a newly developed direct pressure-driven model (i.e., SIPDM) that is based on using a specific set of elements added to each deficient pressure demand node in the network.

In the next chapter the performance of the pressure driven model (PDM) presented in section 3.5 is tested, verified and demonstrated under different flow conditions on a number of case studies. The results obtained are also compared to the results reported/obtained by using a number of reviewed PDM approaches from the literature. 


\section{CHAPTER 4: CASE STUDIES ON PRESSURE-DRIVEN MODEL}

\subsection{Introduction}

The pressure-driven model (SIPDM) presented in Chapter 3 has been tested, verified and illustrated under different flow conditions on a number of benchmarks and real-life case water distribution systems (WDSs) in this chapter. The case studies considered are divided into four groups based on the complexity of the WDSs analysed. The results obtained are also compared to the results reported or obtained by using a number of reviewed PDM benchmarking methods (or obtained by the DDM method where direct comparison was not possible) with the aim of assessing the overall performance and convergence of the SIPDM approach.

This chapter is organized as follows. After this introduction, the details of software and assumptions used are presented in section 4.2. Next, SIPDM approach is tested on a simple small looped network under steady-state analysis, and the obtained results are compared to a number of existed PDM methods. In section 4.4, the SIPDM is demonstrated on more complex and large networks under steady-state analysis. The ability of the SIPDM approach to perform extended period simulation (EPS) is demonstrated on a simple network in section 4.5. Once this done, section 4.6 provides detailed results obtained for EPS analysis on a real-life network under two flow scenarios. Finally, in section 4.7, the entire chapter is summarized.

\subsection{Assumptions and Software}

All hydraulic simulations were conducted by using EPANET2.0 and its toolkit functions for steady-state and EPS analyses. The process of adding the artificial elements to the demand nodes with pressure-deficient conditions requires making changes in the EPANET2.0 input file. A program script written in MATLAB is used with the EPANET2.0 programmers' toolkit to do this in the first part A of the program script. The modified data file can be used to perform steady state analysis using EPANET2.0 Graphical User Interface (GUI). Part B of the program 
is then used to carry out the steady-state analysis and extended period simulation. However, additional toolkit operations are further required to modify the relevant emitter coefficients and FCV settings based on the current demand value.

The diameter, Hazen-Williams roughness coefficient and length for the added artificial CVs and FCVs to pressure-deficient demand nodes (also where added to demand nodes with sufficient-pressure for comparison purpose) are assumed to be equal to $1.0 \mathrm{~m}, 130 \mathrm{~m}$ and $0.1 \mathrm{~m}$ respectively. These values have been chosen in order to neglect the friction head-losses between each demand node and the corresponding added artificial emitter to it (this was illustrated earlier in section 3.5.1 in Chapter 3). Note, that the Darcy-Weisbach roughness coefficient of $0.0001 \mathrm{~mm}$ is used instead of Hazen-Williams coefficient where DarcyWeisbach formula applied.

All simulations are conducted on a computer with the Intel $(R)$ processor, Core (TM) i5-4570 CPU @ $3.2 \mathrm{GHz}$ and 64-bit Windows 7. The default values of accuracy parameters in EPANET2.0, CHECKFREQ, MAXCHECK, and DUMPLIMIT are $0.001,2,10$ and 0 , respectively. It should be noted that even though EPANET2.0 was used in all case studies here, the SIPDM method is generic in a sense that it can be replicated using any other existing hydraulic solvers.

\subsection{Case Study 1: Steady-State Simulation in a Simple Looped Network}

\subsubsection{Network description}

The first network analysed is from the paper by Ang and Jowitt (2006) (see Figure 4.1). This network was used by a number of researchers as a benchmark problem to validate their PDM methods. This network consists of a fixed head reservoir (i.e., source) feeding six demand nodes through eight pipes in a looped configuration. The properties for the pipes and nodes are given in Table 4.1. The value for the empirical exponent coefficient $\left(n_{n}\right)$ that introduced in Equation 3.14 for all demand nodes is assumed to be equal to 1.85. Also, the value for minimum nodal pressure threshold level in the same equation (i.e., $P_{\min }$ ) is assumed to be 
$50 \mathrm{~m}$ at nodes $\mathrm{N} 1$ and $\mathrm{N} 2,45 \mathrm{~m}$ at nodes $\mathrm{N} 3$ and $\mathrm{N} 4$ and $55 \mathrm{~m}$ at nodes $\mathrm{N} 5$ and N6. And finally, the required threshold pressure (i.e., $P_{\text {req }}$ ) in the node head-flow relationship is assumed equal to $60 \mathrm{~m}$ for all demand nodes.

The network is examined under two fictional scenarios: normal network flow condition and a failure in one of the pipes in the system (i.e., closing pipe P3). Thirty-six source head levels between $40 \mathrm{~m}$ and $110 \mathrm{~m}$ were used to demonstrate the influence of system pressures on nodal outflows for each scenario using EPANET2.0 and SIPDM approach. Also for further performance demonstration purpose in term of the accuracy, results converge and computational performance, SIPDM method is compared to results reported/obtained by Ackley et al. (2001), Hayuti et al. (2008), Babu and Mohan (2012), Gorev and Kodzhespirova (2013), and Sayyed et al. (2015) methods under normal flow condition for source head level $=60 \mathrm{~m}$.

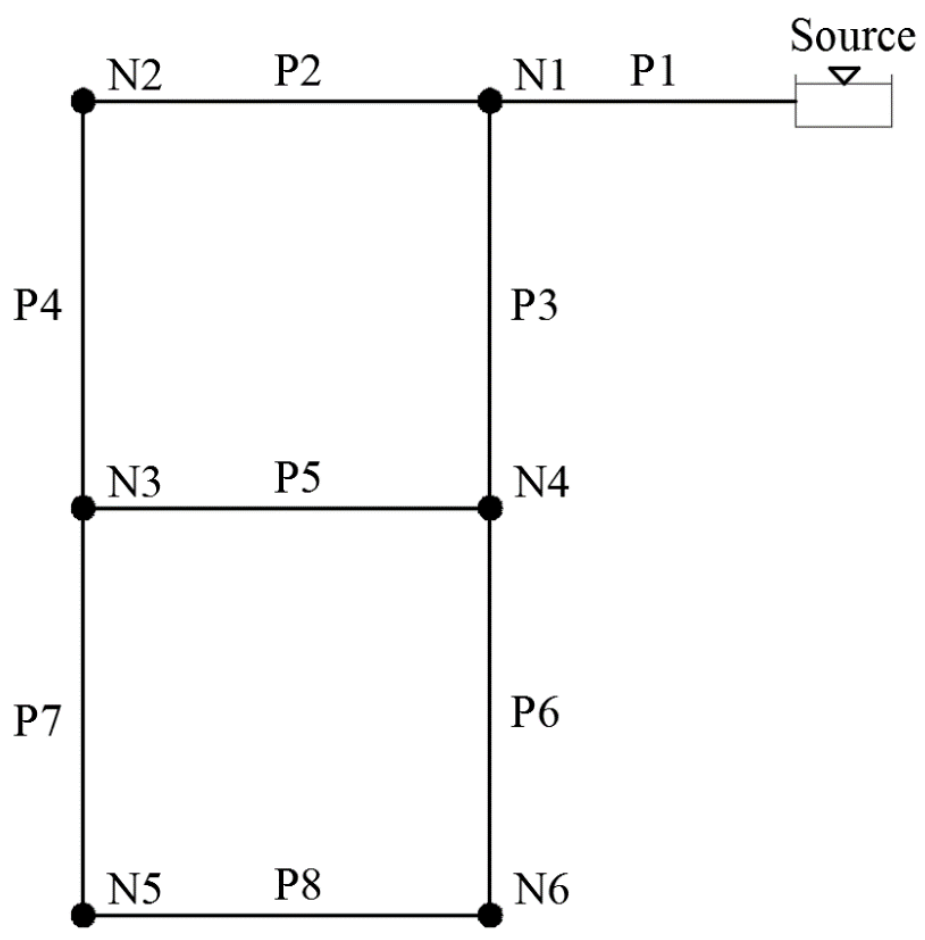

Figure 4.1. Network layout for case study 1. 
Table 4.1. Properties for (a) nodes (b) pipes for the network shown in Figure 4.1.

(a)

\begin{tabular}{|c|c|c|c|c|}
\hline $\begin{array}{c}\text { Node } \\
\text { ID }\end{array}$ & $\begin{array}{c}\text { Elevation } \\
(\mathrm{m})\end{array}$ & $\begin{array}{c}\text { Demand } \\
(\mathrm{Ips})\end{array}$ & $\begin{array}{c}\mathrm{P}_{\text {min }} \\
(\mathrm{m})\end{array}$ & $\begin{array}{c}\mathrm{P}_{\text {req }} \\
(\mathrm{m})\end{array}$ \\
\hline $\mathrm{N} 1$ & 0 & 41.70 & 50 & 60 \\
\hline $\mathrm{N} 2$ & 0 & 41.70 & 50 & 60 \\
\hline $\mathrm{N} 3$ & 0 & 41.70 & 45 & 60 \\
\hline N4 & 0 & 77.80 & 45 & 60 \\
\hline N5 & 0 & 88.90 & 55 & 60 \\
\hline N6 & 0 & 55.60 & 55 & 60 \\
\hline Source & Vary & -347.40 & & \\
\hline
\end{tabular}

\subsubsection{Results and discussion}

A total of 72 DDM (i.e., EPANET2.0) runs, resulting from the two scenarios and 36 head levels at source, were conducted on the network. Those runs were chosen to create different pressure conditions at all network nodes and later to show the performance and capability of SIPDA for predicting nodal pressures outflows under these condition. Figure 4.2 shows the results of the pressure heads at each network node for all runs. It can be observed from the figure that all network nodes under normal flow condition scenario produced three types of pressure conditions. First, the deficient pressure condition (i.e., pressures below the minimum threshold level) under the low-pressure condition in the network (i.e., low head levels at source). Second, the sufficient-pressure condition (i.e., pressures above the required threshold level) for the high head levels at the source. Finally, the partial pressure condition (i.e., pressures between two threshold pressure levels) for the medium head levels at the source. For example, the available pressure head at node $\mathrm{N} 3$ is below the minimum pressure threshold value $50 \mathrm{~m}$ (i.e., $40 \mathrm{~m}$ ) for source head level $50 \mathrm{~m}$, whilst it is about $70 \mathrm{~m}$ (e.g., above the required threshold level $60 \mathrm{~m}$, for source head level $80 \mathrm{~m}$, and it is 45 $\mathrm{m}$ for source head level $60 \mathrm{~m}$. On the other hand, under closed pipe P3 scenario 
all network nodes except $\mathrm{N} 1$ are subjected to more severe low-pressure conditions for all head levels at source in comparison with the normal flow condition (i.e., nodal pressure heads drop further) as shown in Figure 4.2.

As expected the initial DDM runs predicted the available (i.e., actual) flows equal to the required values ( $\left.Q_{r e q}\right)$ in all nodes for all runs regardless to available pressure head value at the nodes. Hence, the results obtained for nodal outflows are correct only when the $P_{a v l}$ is equal or greater than required threshold value (i.e., Preq $=60 \mathrm{~m}$ ). The SIPDM approach is introduced to the network at this point by adding the proposed sequence of artificial elements (shown in Figure 3.8 with the corresponding properties shown in Table 3.6) to the nodes with pressure less than $P_{\text {req }}=60 \mathrm{~m}$. For example, for head source $80 \mathrm{~m}$, the artificial elements are added only to nodes N5 and N6 for the normal flow condition scenario while, for the same source head value, artificial elements are added to all nodes except node N1 under the closed pipe P3 scenario, as shown in Figure 4.1. Once the artificial elements are added, the DDM solver is re-run.
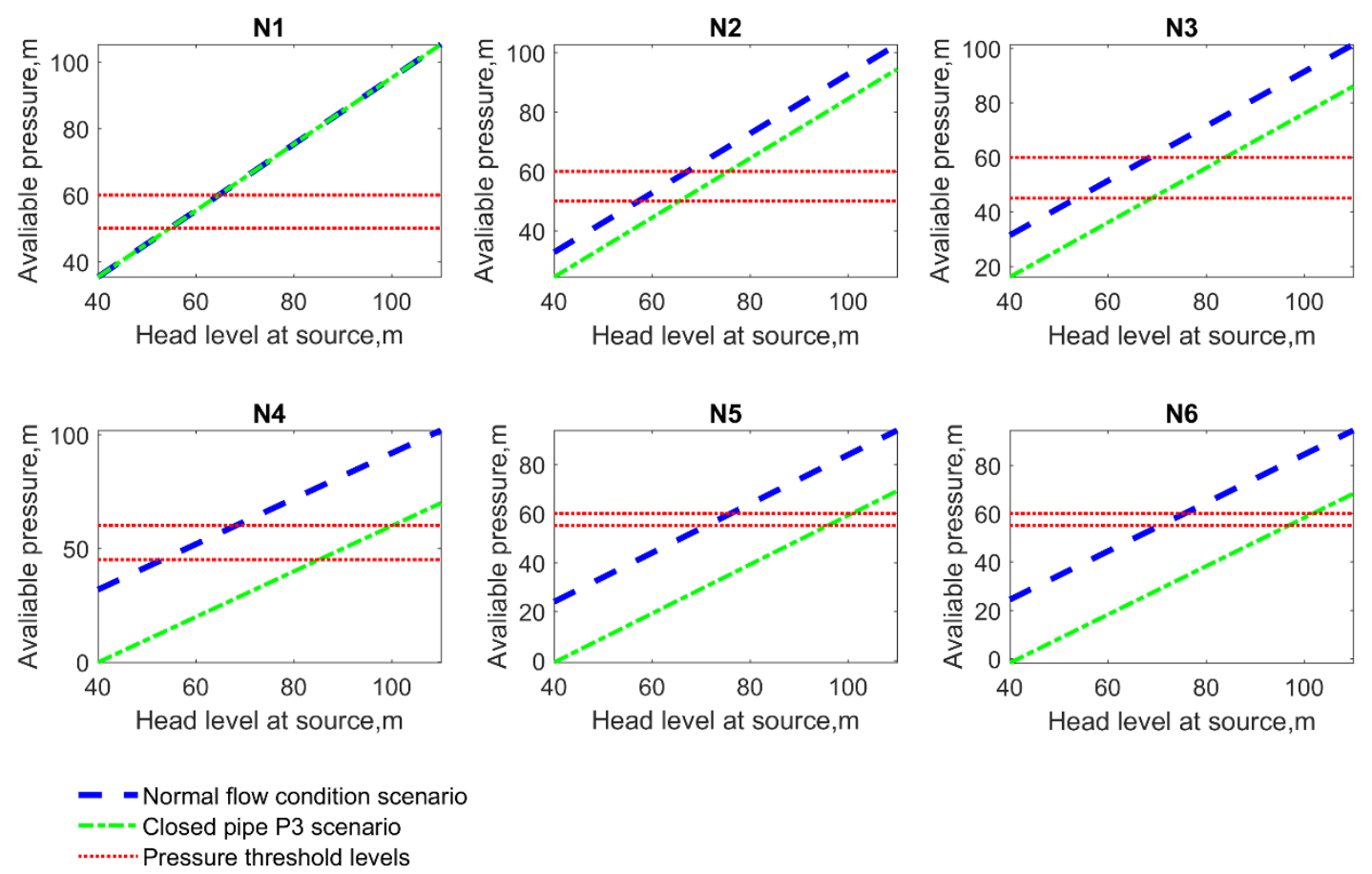

Figure 4.2. Results of DDM analysis on the network shown in Figure 4.1. 
SIPDM approach was successfully applied on the network and the method has converged in limited EPANET2.0 solver iterations (i.e., varying between 5 and 20) resulting in a balanced system for all 72 runs. Figure 4.3 shows source head values obtained versus the SPI ratio of water delivered $\left(Q_{d e l}\right)$ to the water required $\left(Q_{r e q}\right)$ for each network node (dashed blue lines). The secondary vertical axis in the same figure indicates the values of available pressure head and threshold pressure level at each node (dashed red lines).

It can be observed from Figure 4.3 that under the normal flow condition scenario, the outflows from nodes N1-N4 start (i.e., SPI > 0) when the head at source is equal to the minimum pressure threshold level at respective node. The same cannot be said for nodes N5 and N6. This is because these two nodes are located far from the water source compared to other nodes, consequently, more system friction losses which require more head at the source to initiate the flow at these two particular nodes. Also, the SIPDM method delivers a partial demand (i.e., 0 $<\mathrm{SPI}<1$ ) to network nodes at different source heads, when the pressure head values are between the two characteristic threshold levels at each node as shown in Figure 4.3. In addition, from the same figure it can be seen that the relationship between head at source and SPI at nodes in the partial water delivery stage agrees well with the common Wagner et al. (1988) function for $n_{n}=1.85$. For example, partial flow occurs at node N3 when head level at source varies between 45 and $70 \mathrm{~m}$. The demands at nodes N5 and N6 are satisfied (i.e., SPI $=1$ ) only when the head level at source is about $85 \mathrm{~m}$ or higher. 

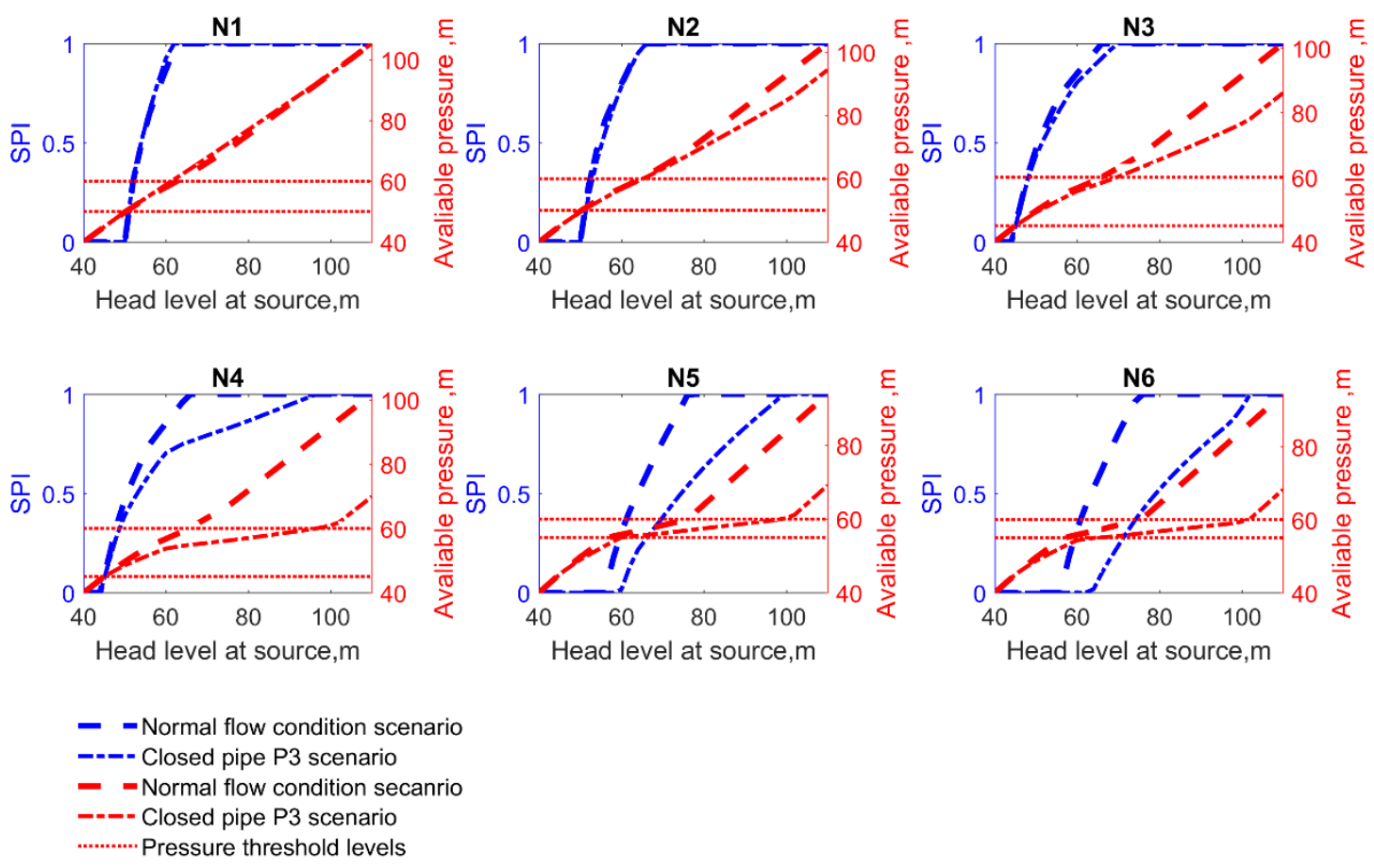

Figure 4.3. Results of SIPDM analysis on the network shown in Figure 4.1.

For the case of isolating pipe P3, the SIPDM shows that the order and commencement of nodal outflows are again not particularly intuitive. For example, the outflow at node $\mathrm{N} 6$ only begins at a source head of $65 \mathrm{~m}$ which is considerably higher than the normal flow condition case (i.e., first scenario). Referring back to Figure 4.1, it can be observed that pipe P3 is a critical pipe linking node $\mathrm{N} 6$ to the source, which explains the required higher source head.

Another interesting observation from the Figure 4.3 is when pipe P3 is isolated, which reveals that the outflow at nodes N4, N5 and N6 meet the respective required demands at a higher source head level compared to the normal network flow condition scenario. This can be explained by the fact that the outflows at N4, $\mathrm{N} 5$, and $\mathrm{N} 6$ are provided by P4 only. This make the path flow line to these nodes longer and put more stress on pipe P4 compared to the normal flow condition where the outflows were provided by both $\mathrm{P} 3$ and $\mathrm{P} 4$.

For further demonstration, Table 4.2 shows the results obtained by the SIPDM method in comparison with methods reported by Ackley et al. (2001), Hayuti et al. (2008), Babu and Mohan (2012), Gorev and Kodzhespirova (2013), and Sayyed et al. (2015) for head level $60 \mathrm{~m}$ at source under normal flow condition scenario. 
Table 4.2. Comparison of the results obtained for case study 1 network under normal flow condition for the source head $60 \mathrm{~m}$.

\begin{tabular}{|c|c|c|c|c|c|c|c|c|c|c|c|c|}
\hline \multirow{4}{*}{ Node Id. } & \multirow{2}{*}{\multicolumn{2}{|c|}{ DDM Results, F }} & \multicolumn{10}{|c|}{ PDM Results } \\
\hline & & & \multicolumn{2}{|c|}{$A$} & \multicolumn{2}{|c|}{ B } & \multicolumn{2}{|c|}{$\mathrm{C}$} & \multicolumn{2}{|c|}{$\mathrm{D}$} & \multicolumn{2}{|c|}{$E$} \\
\hline & $Q_{\text {del }}$ & $P_{\text {avl }}$ & $Q_{\text {del }}$ & $P_{\text {avl }}$ & $Q_{\text {del }}$ & $P_{\text {avl }}$ & $Q_{\text {del }}$ & $P_{\text {avl }}$ & $Q_{\text {del }}$ & $P_{\text {avl }}$ & $Q_{\text {del }}$ & $P_{\text {avl }}$ \\
\hline & $1 / \mathrm{s}$ & $\mathrm{m}$ & $1 / \mathrm{s}$ & $\mathrm{m}$ & $1 / \mathrm{s}$ & $\mathrm{m}$ & $1 / \mathrm{s}$ & $\mathrm{m}$ & $1 / \mathrm{s}$ & $\mathrm{m}$ & $1 / \mathrm{s}$ & $\mathrm{m}$ \\
\hline N1 & 41.7 & 54.33 & 35.0 & 57.23 & 34.1 & 56.88 & 35.0 & 57.22 & 41.7 & 50.00 & 35.4 & 57.19 \\
\hline N3 & 41.7 & 50.40 & 35.2 & 55.98 & 29.5 & 55.26 & 35.2 & 55.98 & 41.7 & 45.00 & 35.6 & 55.92 \\
\hline N4 & 77.8 & 50.83 & 66.0 & 56.06 & 55.9 & 55.42 & 66.0 & 56.06 & 77.8 & 45.00 & 66.6 & 56.00 \\
\hline N5 & 88.9 & 42.91 & 22.5 & 55.39 & 46.4 & 53.00 & 22.5 & 55.39 & 13.4 & 55.00 & 22.6 & 55.32 \\
\hline N6 & 55.6 & 43.40 & 14.8 & 55.43 & 29.7 & 53.14 & 14.8 & 55.43 & 15.1 & 55.00 & 15.0 & 55.36 \\
\hline
\end{tabular}

Notes: A= SIPDM approach; B= Ackley et al. (2001) approach; C=Hayuti et al. (2008) approach; $D=$ Babu and Mohan (2012) approach; $E=$ Gorev and Kodzhespirova (2013) approach; F= Sayyed et al. (2015) approach; Italic numbers indicate partial flow and bold numbers indicate nodes with heads below the minimum pressure head limit, $P_{\min }$; CT= computational time; CTs not available for methods $\mathrm{B}$ and $\mathrm{C}$ due to the complexity of obtaining these values by usingboth PDM approaches. 
SIPDM and Hayuti et al. (2008) approaches outperform the Ackley et al. (2001), Gorev and Kodzhespirova (2013), and Babu and Mohan (2012) methods in terms of prediction accuracy. This can be seen in Table 4.2 by comparing the values of 206.0, 206.1 226.6, 208.2, and $231.8 \mathrm{l} / \mathrm{s}$, respectively, for the total flow (i.e., total demand) drawn from the source. Also, all methods except Babu and Mohan (2012) predict partial delivery at all network nodes. This is because the solutions estimated by the SIPDM and Hayuti et al. (2008) approaches produce pressure dependent nodal flows similar to those derived by using the well validated Wagner et al. (1988) model. While, the value of $n_{n}$ for this network is 1.85 , the assumption in Gorev and Kodzhespirova's (2013) paper causes errors in calculation of nodal flows and pressure in heads. On the other hand, the Babu and Mohan (2012) method cannot model the partial flow as it is based on the $P_{\min }$ only (i.e., it does not consider $P_{\text {req }}$ ), this leave only two nodes with partial delivery as given in Table 4.2 .

SIPDM outperforms the EPANET2.0, Ackley et al. (2001), and Sayyed et al. (2015) methods in terms of convergence/numerical stability. This can be seen in Table 4.2 by comparing the values of nodal outflows (i.e., Qdel) and pressures

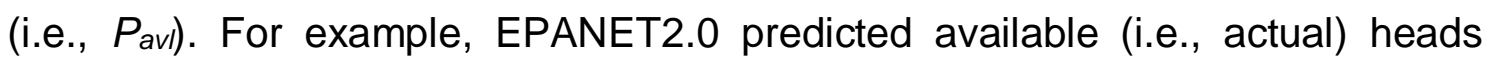
below the required threshold value $\left(P_{\text {req }}\right)$ in all nodes. Hence, the DDM results reported for the failure scenario are meaningless as the demand at nodes cannot be met in full. On the other hand, the Ackley et al. (2001) method generated unrealistic results, in which the pressure head at nodes N5 and N6 were below the minimum $P_{\min }=55 \mathrm{~m}$. This could be because a formal optimization technique was used in this method to maximize nodal flows and the NHFR function was considered as an additional constraint. Finally, the Sayyed et al. (2015) approach failed to change the demand-driven conditions to pressure-driven conditions for all nodes, as shown in Table 4.2 (column 2 and 3), even though they both used the same artificial elements as were used in SIPDM. This is because Sayyed et al. (2015) approach assumes that the elevation of the connected dummy nodes and emitters were equal to the elevation of demand nodes regardless of the minimum pressure head value $\left(P_{\min }\right)$. The above assumption causes errors in pressures and outflow calculations for all nodes, where the connected emitters have $P_{\min }$ values greater than zero, as shown in Table 4.2. 
Finally, SIPDM outperforms the Hayuti et al. (2008), Babu and Mohan (2012), and Gorev and Kodzhespirova (2013) methods in terms of computational efficiency. This can be seen again in Table 4.2. Where SIPDM approach needs only a single internal iteration in EPANET2.0 to converge, as compared to the 10 iterations used by Hayuti et al. (2008). Also, the SIPDM approach obtained the solution quicker than the Babu and Mohan (2012), and Gorev and Kodzhespirova (2013) methods by $14 \%$ and $23 \%$, respectively.

\subsection{Case Study 2: Steady-State Simulation in a Large Pipe Networks}

This case study assess and compares the performance of the SIPDM approach to other PDM approaches on large real-life pipe networks under steady-state simulation. To achieve this, a number of WDSs of different sizes and with different failure scenarios are considered. The results obtained are compared to those produced by using the DDM built in EPANET2.0; and the PDM methods of Babu and Mohan (2012), Gorev et al. (2013), and Sayyed et al. (2015) in terms of prediction accuracy, convergence and computational time. For the purpose of comparison, the artificial sequence of elements proposed in this thesis is connected randomly to $30 \%, 60 \%$ and $100 \%$ of non-zero demand nodes in networks NW3 and NW4 (where no deficient pressure conditions were found), but only connected to nodes with pressure less than the required threshold level (i.e., $P_{a v 1}<P_{\text {req) }}$ in NW1 and NW2. The details of these networks and failure scenarios created are given in the following sections 4.4.1 and 4.4.2.

\subsubsection{Networks description}

The first network, referred to as NW1, is Network 2 from the Battle of the Water Sensor Networks (Ostfeld et al. 2008). This network is considered as a large and challenging real life network in the literature. The NW1 serves 250,000 people over an area of $490 \mathrm{~km}^{2}$. The network model contains 12,523 nodes and 13 hydraulic controls (2 reservoirs, 2 tanks, 4 pumps, and 5 valves) as shown in Table 4.3. The elevation of nodes range from 0 to $74 \mathrm{~m}$. There are 10,552 nonzero demand nodes in the model, and their base demands range from 0 to $16 \mathrm{l} / \mathrm{s}$. The network model was analysed under two pressure deficient conditions: (i) the 
current demand stress conditions (referred here as scenario one) and (ii) large burst on pipe LINK-7496 (referred here as scenario two).

The Exeter network, referred as to NW2, (Farmani et al. 2005) resembles a large real-life reinforcement problem in a water distribution system with a single loading. The network serves a population of approximately 400,000. It has 1891 nodes of which five are source nodes and 283 have no demand. Two of the source nodes have constant heads. The network consists of relatively small pipes and few transmission mains, with a large head-loss range at the extremities of the system, making it highly sensitive to demand increases. The deficient pressure condition in the network was created using demands projected to the year 2020 (i.e., subjecting the existing network to excess demands).

The Modena network, referred to here as NW3 (Wang et al. 2014), includes 317 pipes, 268 demand nodes and 4 reservoirs with fixed head within $72.0 \mathrm{~m}$ to 74.5 $\mathrm{m}$. The pipe material is cast iron. A uniform Hazen-Williams roughness coefficient of 130 is applied to all pipes. On the other hand, the Pescara network, referred here as NW4 (Wang et al. 2014), includes 99 pipes, 68 demand nodes, and 3 reservoirs with fixed head within $53.08 \mathrm{~m}$ to $57.00 \mathrm{~m}$. The pipe material and Hazen-Williams roughness coefficient are the same as Modena network. Both networks are analysed under sufficient pressure condition.

The EPANET2.0 input files for all network models can be downloaded from the Centre for Water Systems at the University of Exeter (CWS 2018). The value for the minimum pressure head $\left(P_{\min }\right)$, required pressure head $\left(P_{\text {req }}\right)$, and $n_{n}$ for demand nodes in each network model are summarized in Table 4.3. The table also shows the type of head-loss equation used in each network model. 
Table 4.3. Characteristics of network models used in the performance tests for case study 2.

\begin{tabular}{|c|l|c|c|c|c|c|c|c|c|}
\hline $\begin{array}{c}\text { Network } \\
\text { ID }\end{array}$ & $\begin{array}{l}\text { Network } \\
\text { name }\end{array}$ & $\begin{array}{c}\text { Num. of } \\
\text { Nodes }\end{array}$ & $\begin{array}{c}\text { Num. } \\
\text { of } \\
\text { Pipes }\end{array}$ & $\begin{array}{c}\text { Num. of } \\
\text { Reservoirs }\end{array}$ & $\begin{array}{c}\text { Head-loss } \\
\text { Equation }\end{array}$ & $\begin{array}{c}\mathrm{n}_{\mathrm{n}} \\
\mathrm{P}_{\text {min }} \\
\mathrm{m}\end{array}$ & $\begin{array}{c}\mathrm{P}_{\text {req }} \\
\mathrm{m}\end{array}$ & $\begin{array}{c}\text { Total required } \\
\text { demand } \\
(\mathrm{I} / \mathrm{s})\end{array}$ \\
\hline NW1 & BWSN & 12,523 & 14,830 & 7 & D-W & 2.0 & 0 & 20 & $1,463.86$ \\
\hline NW2 & Exeter & 1,891 & 3,032 & 2 & D-W & 1.5 & 0 & 20 & $3,245.81$ \\
\hline NW3 & Modena & 268 & 317 & 4 & H-W & 2.0 & 7 & 20 & 406.93 \\
\hline NW4 & Pescara & 68 & 99 & 3 & H-W & 2.0 & 7 & 25 & 257.40 \\
\hline
\end{tabular}

Note: $\mathrm{D}-\mathrm{W}=$ Darcy-Weisbach; H-W = Hazen-Williams. 


\subsubsection{Results and discussion}

To check the accuracy of the reported results using the SIPDM approach under sufficient-pressure network conditions, the EPANET2.0 solver was applied to networks NW3 and NW4 in which the DDM assumption (i.e., no pressure deficient nodes) is satisfied.

Figure 4.4 shows the total errors obtained in pressure and outflow at demand nodes when comparing the results of the SIPDM approach with those of the EPANET2.0 solver. From an analysis of the errors, it can be seen that the SIPDM approach delivers almost the same predictions for pressure and outflow at all nodes in both networks as the EPANET2.0. For example, by adding artificial elements to $30 \%$ of demand nodes in both networks, the total error in nodal outflows is 0.0001 and $0.0004 \mathrm{l} / \mathrm{s}$ for NW3 and NW4, respectively. Where, the total error in nodal pressure head is 0.01 and $0.001 \mathrm{~m}$. However, by connecting artificial elements to more demand nodes (e.g., $60 \%$ and 100\%), the error percentage increased in both total pressure head and outflow (but still within acceptable ranges) as shown in Figure 4.4.
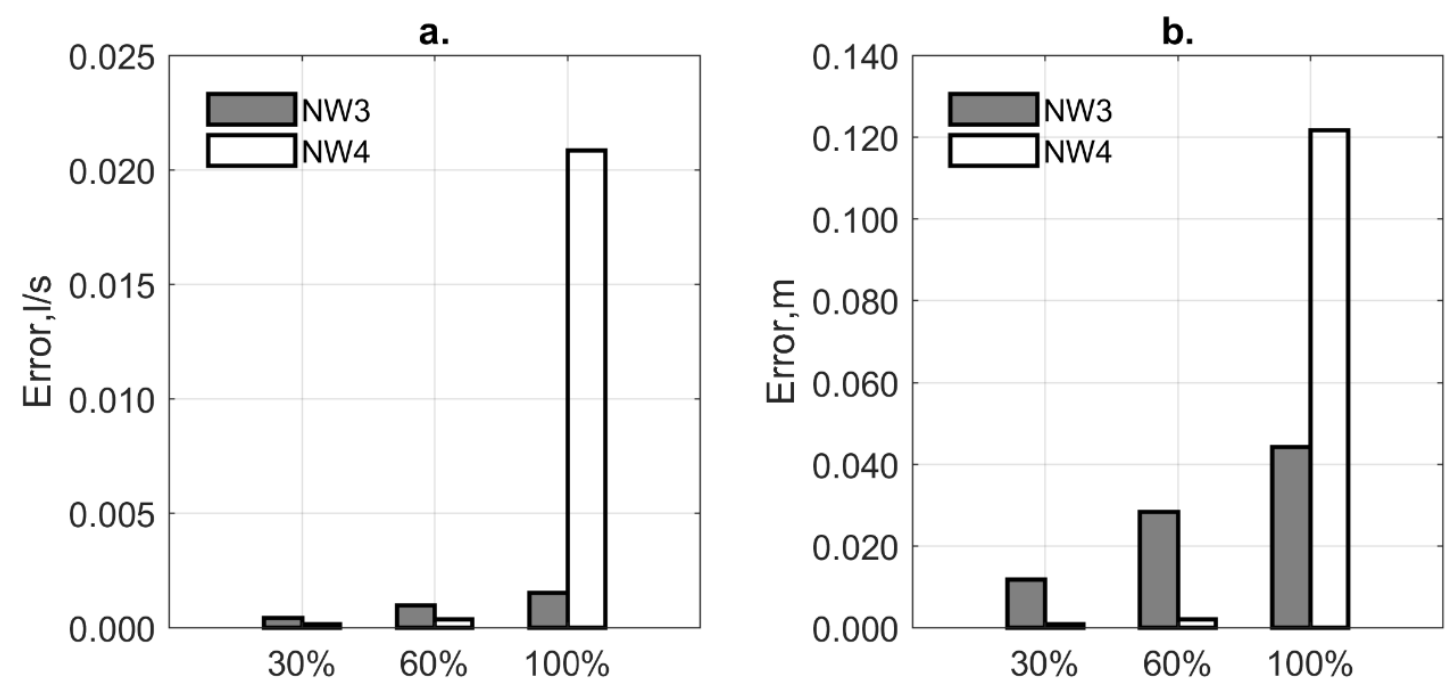

Percentage of demand nodes with artificial elements

Figure 4.4. Errors observed in (a) total outflow (b) pressure head at demand nodes for networks NW3 and NW4. 
Further, the EPANET2.0 solver and SIPDM approach are applied to networks NW1 and NW2 with identical abnormal conditions. The simulation results obtained are shown in Table 4.4. The first three rows show the number of demand nodes for which the pressure is below $P_{\min }$ (zero), the number of nodes with pressures between $P_{\min }$ and $P_{\text {req }}(20 \mathrm{~m})$, and finally the number of nodes with pressures above $P_{\text {req, }}$, respectively, all obtained by using the EPANET2.0 solver. The last three rows show the number of demand nodes in failure mode $\left(P_{a v l}<0\right)$, the number of demand nodes in partial delivery mode $\left(0<P_{a v l}<P_{r e q}\right)$ and the numbers of demand nodes in full delivery mode $\left(P_{a v l} \geq P_{\text {req }}\right)$, all for the case when SIPDM approach is used.

Table 4.4. Comparison of the results observed in networks NW1 and NW2 using EPANET2.0 solver and SIPDM approach.

\begin{tabular}{|l|l|c|c|c|}
\cline { 3 - 5 } \multicolumn{2}{c|}{} & \multicolumn{2}{c|}{ NW1 } & \multirow{2}{*}{ NW2 } \\
\cline { 3 - 5 } \multicolumn{2}{c|}{} & Scenario 1 & Scenario 2 & \\
\hline \multirow{2}{*}{$\begin{array}{l}\text { EPANET2.0 } \\
\text { solver }\end{array}$} & $P_{\text {avl }}<0$ & 0 & 2265 & 97 \\
\cline { 2 - 5 } & $0<P_{\text {avl }}<20$ & 185 & 2402 & 723 \\
\cline { 2 - 5 } & $P_{\text {avl }} \geq 20$ & 10370 & 5887 & 783 \\
\hline \multirow{2}{*}{$\begin{array}{l}\text { SIPDM } \\
\text { Approach }\end{array}$} & $P_{\text {avl }}<0$ & 0 & 619 & 0 \\
\cline { 2 - 5 } & $0<P_{\text {avl }}<P_{\text {req }}$ & 72 & 3684 & 511 \\
\cline { 2 - 5 } & $P_{\text {avl }} \geq P_{\text {req }}$ & 10753 & 6249 & 1092 \\
\hline
\end{tabular}

The results demonstrate the capability of the SIPDM approach in changing the status of demand nodes from failure mode to fully or partially delivery modes and deliver logical results based on the network boundary conditions (e.g., size, topology, failure intensity, location, and pressure) when compared to EPANET2.0. In the case of the DDM (i.e., EPANET2.0) solver, the deficient pressures were observed in both network. Hence, the DDM results reported for the failure scenarios are meaningless because the outflow at nodes with pressure less than $20 \mathrm{~m}$ cannot be met in full. For instance, 820 nodes in NW2 have pressure values below $20 \mathrm{~m}$. In the case of the SIPDM approach, although in all three cases the situation of most demand nodes with deficient pressure were changed to either partial or full delivery mode (as reported in Table 4.4), there are 
some quite marked differences. In network NW2, out of 820 pressure deficient nodes, 511 have partial delivery mode and the rest have the full delivery mode. This is because the most deficient node in network (i.e., 723) has pressure between 0 and $20 \mathrm{~m}$. At the same time, in NW1, under scenario two, where the number of nodes with pressure less than $0 \mathrm{~m}$ are relatively high (i.e., 2265), the SIPDM changed the situation of most of them from failure to partial delivery mode.

Table 4.5 reports further comparison results between SIPDM approach with other PDM methods and EPANET2.0 solver in term of accuracy and computational time performance.

The accuracy was measured through the effect coefficient $n_{n}$ shown in the Equation 3.14 on the NFHR in the Equation 3.16. According to results obtained, the SIPDM approach delivers more realistic predictions for nodal outflow and pressure reflecting the properties of the network nodes. Where, the SIPDM approach and Gorev et al. (2013) method produced the same demand satisfaction ratio, (DSR): the ratio of water delivered to the water required (Siew and Tanyimboh 2012), for networks NW1 (under first scenario), NW3 and NW4 with DSR values of $0.970,1.0$ and 1.0 , respectively. This is because the value of $n_{n}$ in all three networks is 2.0 as given in Table 4.3. However, the SIPDM approach obtained a smaller DSR value of 0.926 for NW2 in comparison with Gorev et al. (2013) method of 0.934. This is because the proposed SIPDM approach uses the Wagner et al. (1988) pressure dependent nodal flow function where $n_{n}=1.5$, whilst the shape of the NHFR function in Gorev et al. (2013) approach is fixed at $n_{n}=2.0$ regardless to the actual value $n_{n}$ (e.g., 1.5) value for nodes. The effect of this assumption on nodal pressures are shown in the Figure 4.5. Also, the same figure highlights the results obtained using Mohan and Babu (2012) and EPANET2.0 methods. The Babu and Mohan (2012) method produced DSR values of 1.0 and $0.988 \mathrm{NW} 1$ and NW2, the reason for this being that their method cannot simulate partial nodal flows. On the other hand, as expected, EPANET2.0 produced unrealistic negative pressure heads and DSR values (i.e., $1.0)$ in NW1 and NW2. 
Table 4.5. Results comparison for (a) DSR (b) computational time performance in Steady-State simulation in a large pipe networks for case study 2.

(a)

\begin{tabular}{|l|c|c|c|c|}
\hline & \multicolumn{5}{|c|}{ DSR } \\
\cline { 2 - 5 } Network & EPANET2.0 & SIPDM & $\begin{array}{c}\text { Babu and Mohan } \\
(2012)\end{array}$ & $\begin{array}{c}\text { Gorev et al. } \\
(2013)\end{array}$ \\
\hline NW1 & 1.0 & 0.97 & 1.0 & 0.97 \\
\hline NW2 & 1.0 & 0.926 & 0.988 & 0.934 \\
\hline NW3 & 1.0 & 1.0 & 1.0 & 1.0 \\
\hline NW4 & 1.0 & 1.0 & 1.0 & 1.0 \\
\hline
\end{tabular}

(b)

\begin{tabular}{|l|c|c|c|c|}
\hline \multirow{2}{*}{ Network } & \multicolumn{4}{|c|}{ Time (s) } \\
\cline { 2 - 5 } & EPANET2.0 & SIPDM & $\begin{array}{c}\text { Babu and Mohan } \\
(2012)\end{array}$ & $\begin{array}{c}\text { Gorev et al. } \\
(2013)\end{array}$ \\
\hline NW1 & $5.60 \times 10^{-3}$ & $5.60 \times 10^{-2}$ & $5.65 \times 10^{-2}$ & $5.75 \times 10^{-2}$ \\
\hline NW2 & $4.60 \times 10^{-4}$ & $1.30 \times 10^{-3}$ & $5.90 \times 10^{-3}$ & $1.05 \times 10^{-2}$ \\
\hline NW3 & $1.35 \times 10^{-4}$ & $3.85 \times 10^{-4}$ & $3.11 \times 10^{-4}$ & $3.98 \times 10^{-4}$ \\
\hline NW4 & $3.20 \times 10^{-5}$ & $8.80 \times 10^{-5}$ & $6.60 \times 10^{-5}$ & $1.71 \times 10^{-4}$ \\
\hline
\end{tabular}


(a)

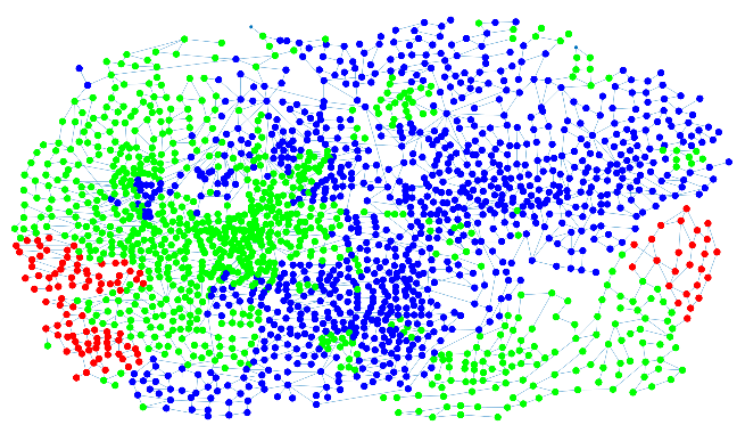

(c)

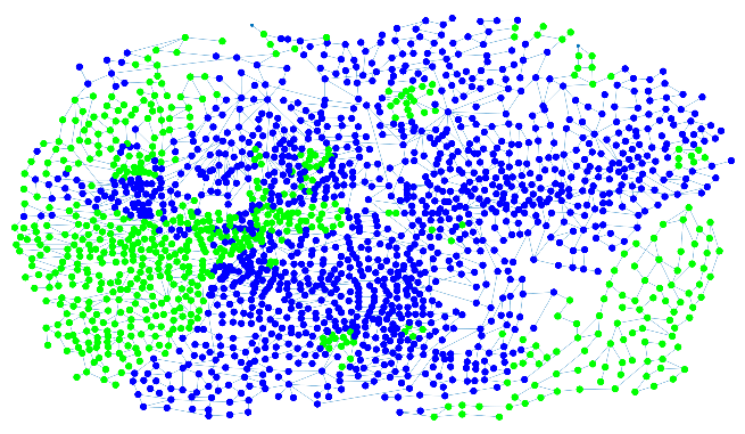

(b)

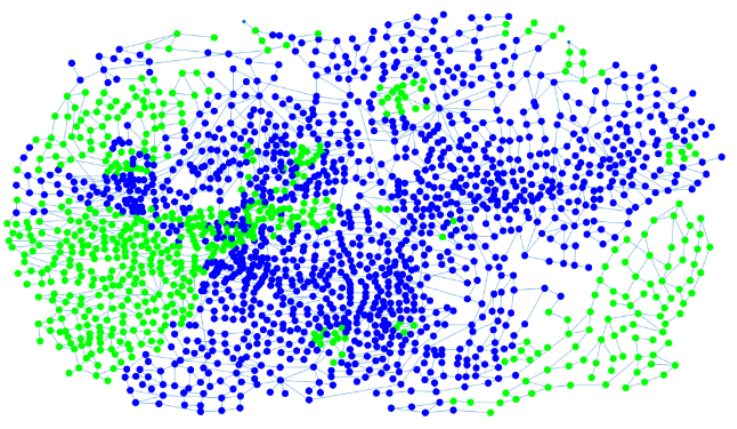

(d)

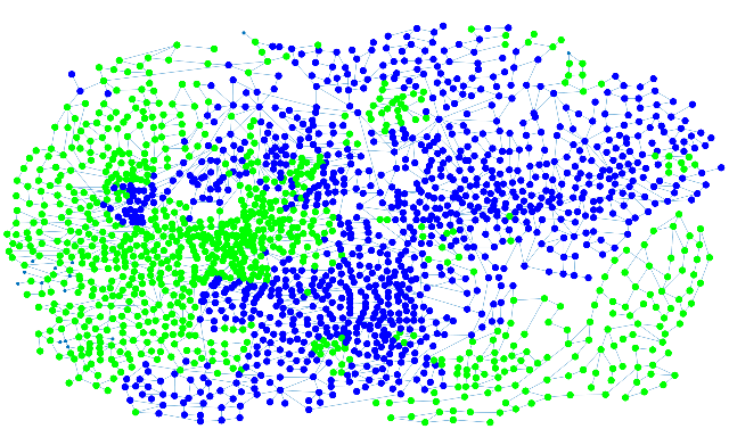

- Nodes with $P_{a v l}<0 \quad$ Nodes with $0<P_{a v l}<20$ - Nodes with $P_{a v l} \geq 20$

Figure 4.5. The pressure values observed for nodes in network NW2 using (a) EPANET2.0 (b) SIPDM approach (c) Gorev et al. (2013) method (d) Babu and Mohan (2012) method.

In terms of computational performance comparison, as expected, EPANET2.0 produced the solutions notably faster than the SIPDM approach for all networks, as shown in Table 4.5. In comparison to the results of Gorev et al. (2013), the SIPDM approach shows a better performance in terms of computational time for all networks, where additional artificial elements are only added to the deficient nodes, and an extra calculation of friction loss in the CV pipes is not required. The computational performance of the SIPDM approach is also better than the Babu and Mohan (2012) approach in large networks (NW2 and NW3). However, the Babu and Mohan (2012) method is faster for smaller network NW4. This is because of the large number of artificial reservoirs that need to be added to all nodal demands in the Babu and Mohan (2012) method, which slows down 
computations increasingly more in larger networks (to obtain relevant water balances between the reservoirs).

Finally, in order to illustrate the advantage of SIPDM over the other approaches which add artificial elements to all network nodes (such as Babu and Mohan 2012; Gorev et al. 2013; Sayyed et al. 2015), the SIPDM and Sayyed et al. (2015) approaches are applied to network NW1 for the first scenario. When the DDM solver (EPANET2.0) is applied to this network it shows that only 185 nodes with demands experience low pressure conditions (i.e., $P_{a v l}<P_{r e q}=20 \mathrm{~m}$ ). Accordingly, in the SIPDM approach, artificial elements are connected only to these nodes, which represent about 1.5\% of the total network of nodes, whilst in Sayyed et al. (2015) artificial elements are added to all 12,523 nodes. As a result, SIPDM is $68 \%$ faster in performing the PDM simulation than the Sayyed et al. (2015) approach. In addition, the Sayyed et al. (2015) approach fails to converge when the above network is exposed to more serious failure scenarios. For example, when a large burst modelled on pipe LINK-7496 (scenario two), EPANET2.0 shows that 2,265 demand nodes are subjected to pressure deficient conditions (i.e. $P_{a v l}<P_{\min }$ ) as shown in Table 4.4. SIPDM successfully changes the status of 1,646 of these nodes from no outflow to partial outflow under these conditions whereas the Sayyed et al. (2015) approach fails to change the status of any of these deficient nodes. Therefore, the Sayyed et al. (2015) approach fails to converge and produces unrealistic predictions of nodal pressures and outflows.

\subsection{Case Study 3: Extended Period Simulation in a Small Network}

The SIPDM approach is applied to a single source pumped WDN with the consideration of a diurnal variation in demands to assess SIPDM performance under EPS conditions. The network was tested under two pressure-deficient conditions. The first condition was obtained by increasing the base demand at all nodes 2.5 times. The value of 2.5 has been chosen to ensure that all demand nodes are subjected to the pressure-deficient condition for some time in the analysed simulation period. The second condition was produced by assuming pipe P7 isolation for the whole simulation period. 


\subsubsection{Network description}

The network consists of a reservoir, a tank, 8 demand nodes and 12 pipes (Figure 4.6). The details associated with the demand nodes and pipes are listed in Table 4.6. Figure 4.6 also provides the location of the 8 isolation valves in the network. The reservoir R1 provides water to the system via pump station shown in the Figure 4.6. The tank connected to pipe P3 completes the model. The initial head level of the tank is $36 \mathrm{~m}$, while the minimum and maximum head level are 31 and $46 \mathrm{~m}$, respectively. Although gravity flow does play a role in supplying flow, pumping is still required. The pump curve is defined by one head versus flow coordinate, $76.2 \mathrm{~m}$ for $94.6 \mathrm{l} / \mathrm{s}$.

Figure 4.7 shows the typical diurnal profile of demand multiplier factors for demand nodes over 24 hours. The simulation time and hydraulic time step are fixed at 24 and $1 \mathrm{hr}$ for all runs. The required pressure head $\left(P_{\text {req }}\right)$, the minimum pressure head $\left(P_{\min }\right)$ and the emitter exponent for demand nodes are assumed to be $20 \mathrm{~m}, 0 \mathrm{~m}$ and 0.54 , respectively.

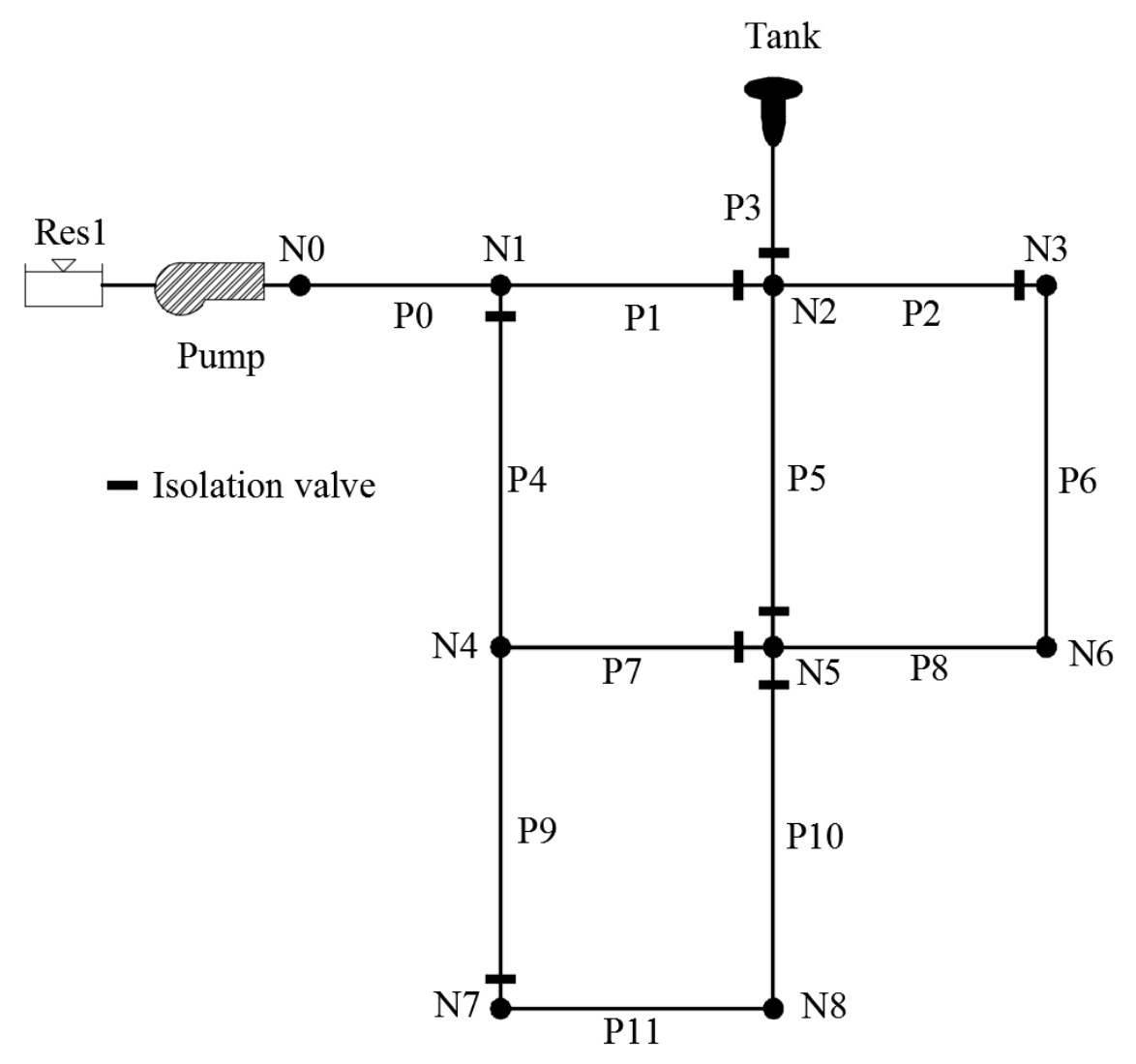

Figure 4.6. Test case study 3 network configuration. 
Table 4.6. Properties for (a) nodes (b) pipes for case 3 network.

(a)

\begin{tabular}{|c|c|c|}
\hline Node ID & $\begin{array}{c}\text { Elevation } \\
(\mathrm{m})\end{array}$ & $\begin{array}{c}\text { Base } \\
\text { demand } \\
(\mathrm{l} / \mathrm{s})\end{array}$ \\
\hline N0 & 216.41 & 0.00 \\
\hline N1 & 216.41 & 9.46 \\
\hline N2 & 213.36 & 9.46 \\
\hline N3 & 211.84 & 6.31 \\
\hline N4 & 213.36 & 9.46 \\
\hline N5 & 211.84 & 12.62 \\
\hline N6 & 210.31 & 9.46 \\
\hline N7 & 213.36 & 6.31 \\
\hline N8 & 216.41 & 6.31 \\
\hline
\end{tabular}

(b)

\begin{tabular}{|c|c|c|c|}
\hline Pipe ID & $\begin{array}{c}\text { Length } \\
(\mathrm{m})\end{array}$ & $\begin{array}{c}\text { Diameter } \\
(\mathrm{mm})\end{array}$ & $\begin{array}{c}\text { Roughness } \\
\text { coefficient }\end{array}$ \\
\hline P0 & 3209.54 & 457.2 & 100 \\
\hline P1 & 1609.34 & 355.6 & 100 \\
\hline P2 & 1609.34 & 254.0 & 100 \\
\hline P3 & 60.96 & 457.2 & 100 \\
\hline P4 & 1609.34 & 254.0 & 100 \\
\hline P5 & 1609.34 & 304.8 & 100 \\
\hline P6 & 1609.34 & 203.2 & 100 \\
\hline P7 & 1609.34 & 254.0 & 100 \\
\hline P8 & 1609.34 & 304.8 & 100 \\
\hline P9 & 1609.34 & 203.2 & 100 \\
\hline P10 & 1609.34 & 152.4 & 100 \\
\hline P11 & 1609.34 & 152.4 & 100 \\
\hline & & & \\
\hline
\end{tabular}

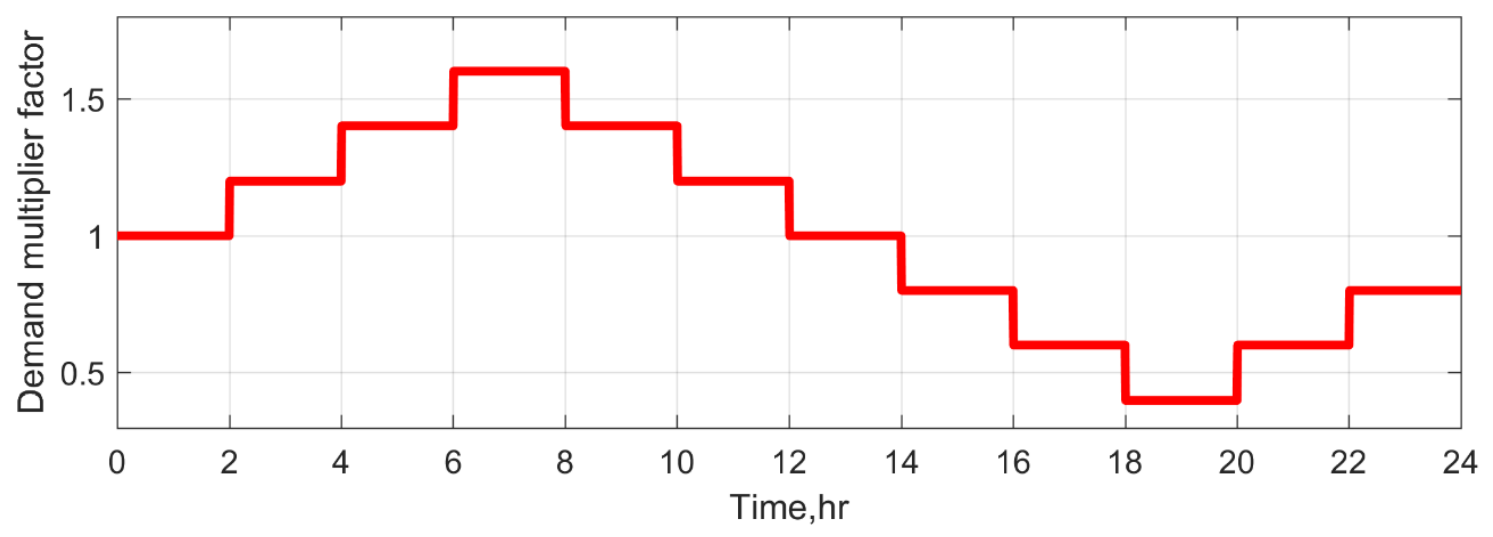

Figure 4.7. Demand multiplier factors for case study 3 network. 


\subsubsection{Results and discussion}

\subsubsection{First pressure-deficient condition - increased demand}

In order to reveal the maximum number of demand nodes with pressure-deficient condition over the analysed simulation period, a $24 \mathrm{~h}$ hydraulic simulation using EPANET2.0 was conducted first. Figure 4.8 shows the results of pressure values with the time at each network node. It can be observed from the figure that all nodes start with sufficient pressure until time 4:00. From this time onward, all nodes exposed to sudden negative pressure and reach to the minimal values at the peak demand times between 6:00 and 8:00. The pressure in the system increase gradually after 8:00 and nodes were receive sufficient pressure from time 14:00 to the end of simulation time (e.g., during the low demand loading time). Thus, the artificial elements are connected to all nodes for the whole simulation period before conducting SIPDM approach. This is important to change the situation of nodes from DD to PD during low pressure condition time, and from PD to DD during sufficient pressure condition time.

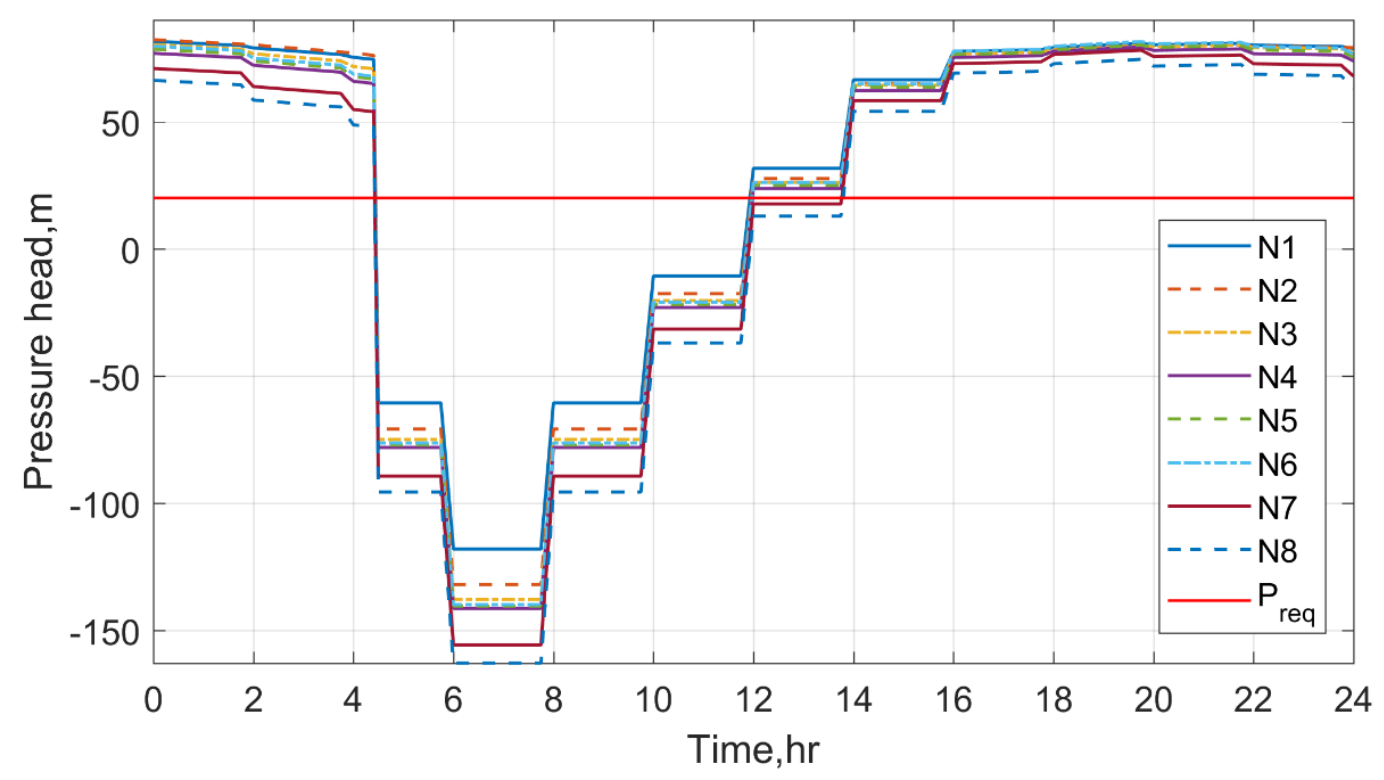

Figure 4.8. Pressure predictions at demand nodes for case study 3 network using EPANET2.0 solver under increased demands condition. 
To achieve this, the extent of demand satisfied at nodes N1 to N8 during the above abnormal flow condition using SIPDM are shown in Figure 4.9. It can be observed from the figure that all nodes are receiving only partial flow during the period from 4:00 to 12:00, when the value of demands are peaked and the pressure values are below $P_{\text {req. }}$. Among these nodes, N1 is less affected by shortfall flow and receives around $85 \%$ of its required demand at time 6:00 to $8: 00$. At the same time, N8 is the worst affected node when only about $30 \%$ of the demand is delivered to it. Also N1 receives full delivery earlier (at 10:00) than N2 through N7 (at 12:00), and N8 at 14:00. This is because N8 is located a far away from the only supply provider R1 in the network from 4:00 14:00 (e.g., no flow from the tank) when compared to $\mathrm{N} 1$ and other nodes as shown in Figure 4.9. All above, reveals the capability of the SIPDM for changing the status of demand nodes between DD and PD during EPS, as well as predicting the more realistic values for flows with time, all based on the pressure, demand and topological conditions in the network.

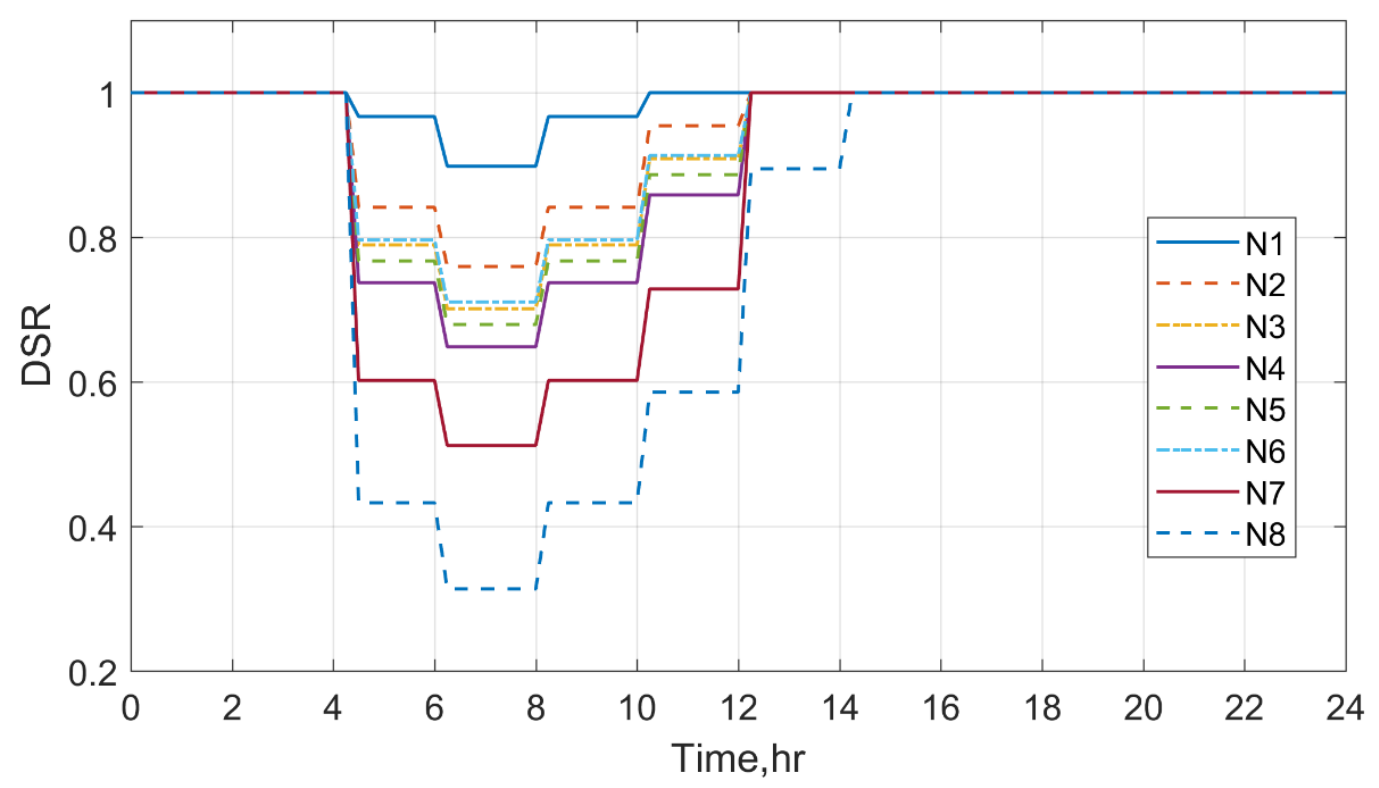

Figure 4.9. Demand satisfaction ratios at demand nodes for case study 3 network using SIPDM approach under increased demands condition. 


\subsubsection{Second pressure-deficient condition - pipe failure}

For demonstration purposes, a failure of $\mathrm{P} 7$ during the whole simulation time is considered to create a pressure-deficient condition. In order to isolate P7, the isolation valves located on P4, P7 and P9 are closed. As a result, P4 and P9 are also isolated from the other parts of the network and demand node N4 disconnected from the water sources (Jun and Loganathan 2007). Note that in the SIPDM approach the settings of the added elements at the isolated demand nodes (e.g., N4) do not need to be reset to zero during hydraulic calculations for the isolated network nodes (which normally needs to be done). However, in order to apply the Ang and Jowitt (2006) and Jinesh Babu approaches for analysing isolation in a WDS, the additional artificial reservoirs should be removed, as they provide water to the isolated parts.

A 24-hour EPS was carried out with a one-hour time step. Demand pattern values were changed every two hours for all nodes. Furthermore, FCV and emitter settings were updated at the beginning of each pattern time step based on the corresponding required nodal demands ( $Q_{\text {req }}$ ) for deficient nodes N7 and N8. These nodes are only exposed to pressure deficient condition, using EPANET2.0 solver as shown in Figure 4.10. While, all nodes in the first scenario were exposed to failure mode conditions as illustrated in section 4.5.2.1.

The results obtained demonstrates that the SIPDM approach provides logical and numerically stable results under EPS conditions which cannot be said for the majority of the other proposed PDM methods in the literature. For instance, the hydraulic simulation of the network by the SIPDM approach shows that shortfall in supply occurred over the whole simulation period, as it can be seen from Figure 4.11. This shortfall increases slightly with the time and reaches the peak of 33.5 $\mathrm{l} / \mathrm{s}$ at 6:00 through 8:00, then drops to around $7.0 \mathrm{l} / \mathrm{s}$ at $18: 00$ and 19:00 and then increases again to $14 \mathrm{l} / \mathrm{s}$ at 23:00, as shown in Figure 4.11. The shortfall is occurring mainly because of the isolation of N4 and partial flows at nodes N7 and N8 during the peak demand times. 


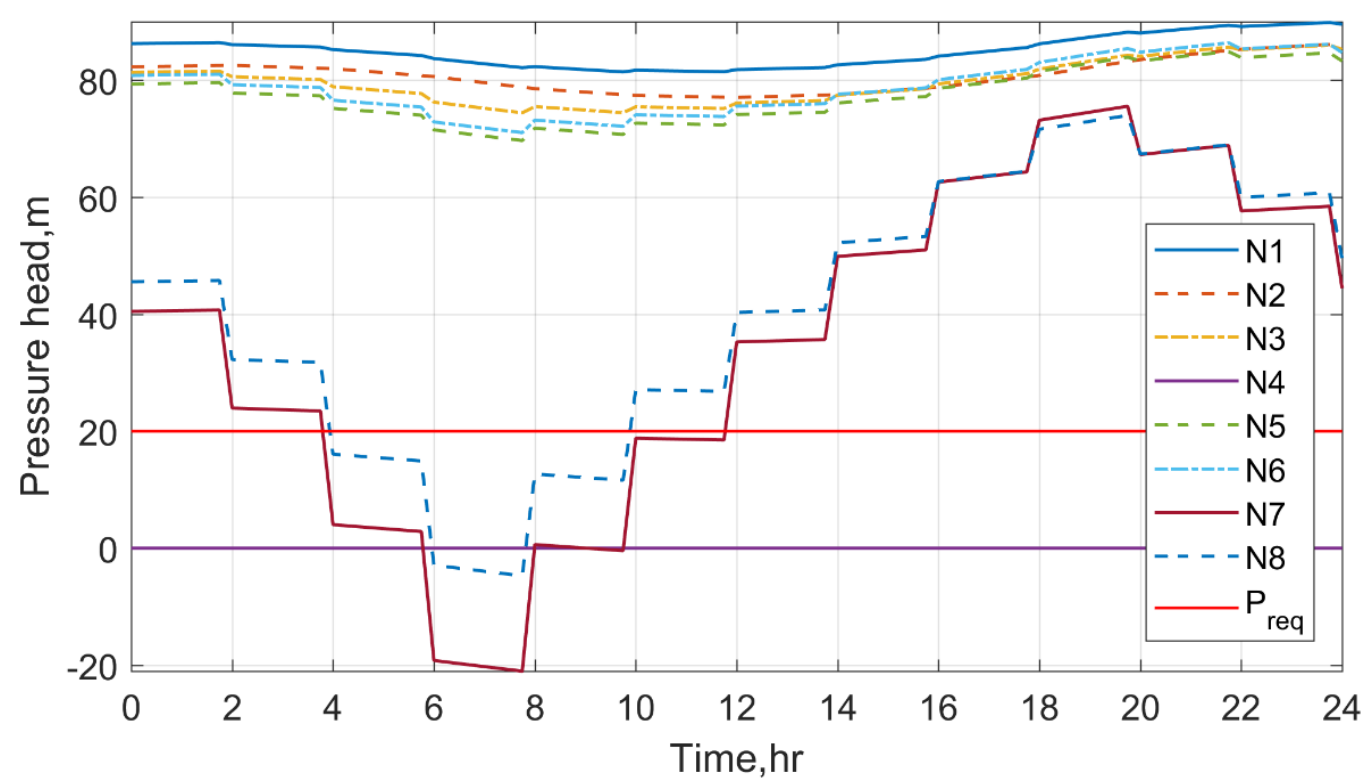

Figure 4.10. Pressure predictions at demand nodes for case study 3 network using EPANET2.0 solver under isolating pipe P7 condition.

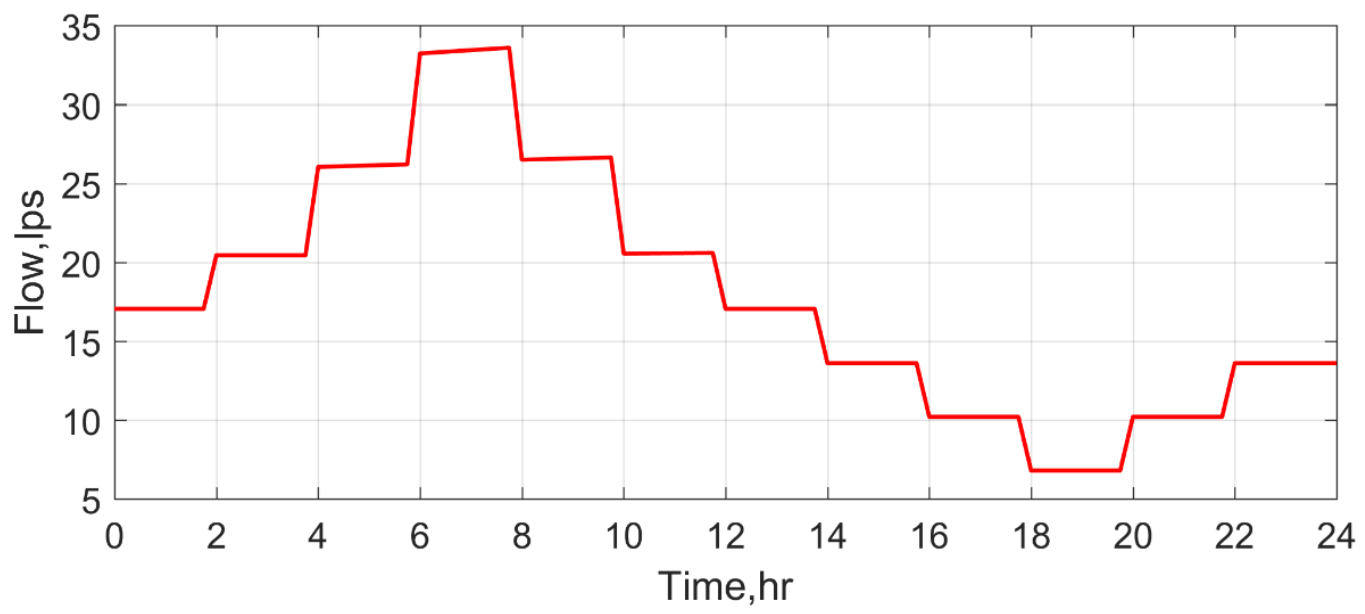

Figure 4.11. Supply shortfall in case study 3 network under isolating pipe P7 condition. 
Further, Figure 4.12 shows that node N7 is unable to deliver the full demand from 4:00 until 12:00 because the pressure head in this period is below the required value $\left(P_{\text {req }}\right)$. However, the pressure head from 12:00 to 24:00 is sufficient to supply the full demand, as shown in Figure 4.12. Consequently, the network experiencing the above failure condition provides the required demand at all nodes during the low demand period but not during the peak-demand period when some pressure heads drop below $P_{\text {req. }}$. All this reveals the ability of SIPDM approach in delivering more realistic and numerically stable results under EPS conditions.

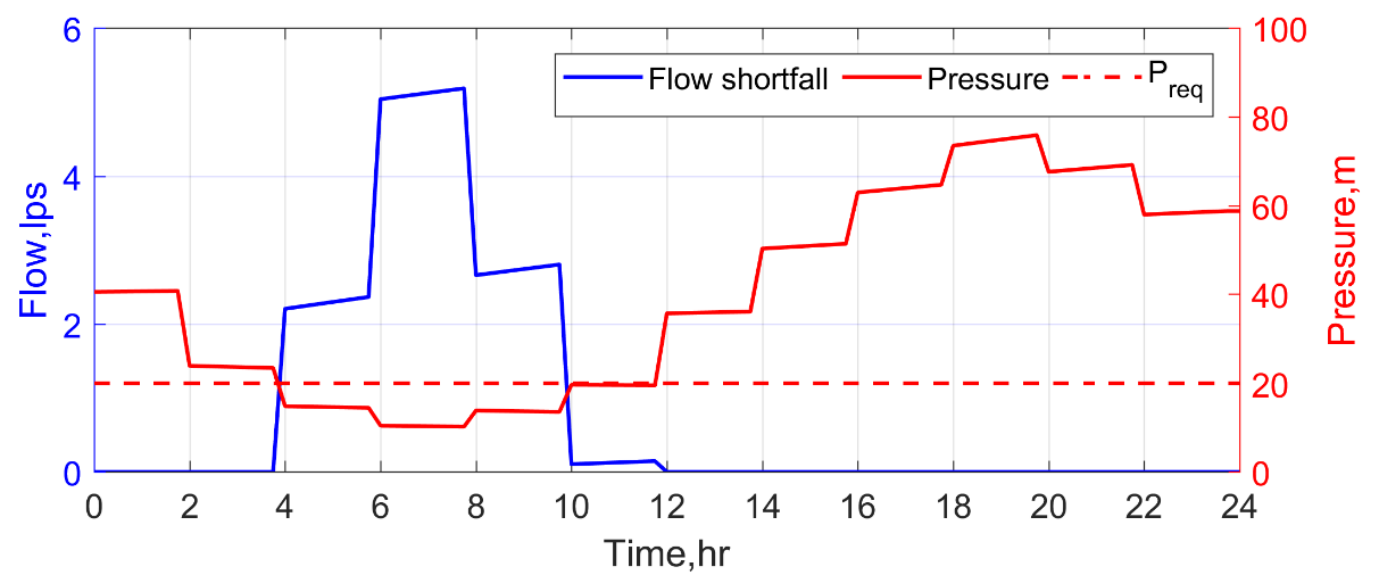

Figure 4.12. Supply shortfall and available pressure head at node N7 in case study 3 network under isolating pipe P7 condition.

\subsection{Case Study 4: Extended Period Simulation in a Large Real- life Network}

In order to further validate the robustness of the SIPDM approach and measure its computational performance, a 24-hour simulation was conducted on a large and more complex WDN, the of C-Town network (Ostfeld et al. 2010). This was done under both normal and abnormal flow conditions simulated in respective scenarios. The results obtained by the SIPDM approach were compared with the DDM (EPANET2.0) hydraulic solver results. 


\subsubsection{Network description}

The C-Town network consists of 6 DMAs, 443 pipes, 399 nodes, 4 PRVs and one TCV. The water is supplied to the system by a large reservoir (RES1) with a constant head and via seven balancing water tanks. The water is pumped through pumping station $\mathrm{S} 1$ to tanks $\mathrm{T} 1$ and $\mathrm{T} 2$, and the water supply to $\mathrm{T} 2$ is controlled by a TCV. Pumping stations S2 and S3 draw water to tanks T3 and T4, while stations S4 and S5 pump supply from T1 to T5, T6 and T7 as shown in Figure 4.13. The required pressure head $\left(P_{\text {req }}\right)$, the minimum pressure head $\left(P_{\min }\right)$ and the emitter exponent for demand nodes are assumed to be $15 \mathrm{~m}$, o $\mathrm{m}$ and 0.5 , respectively.

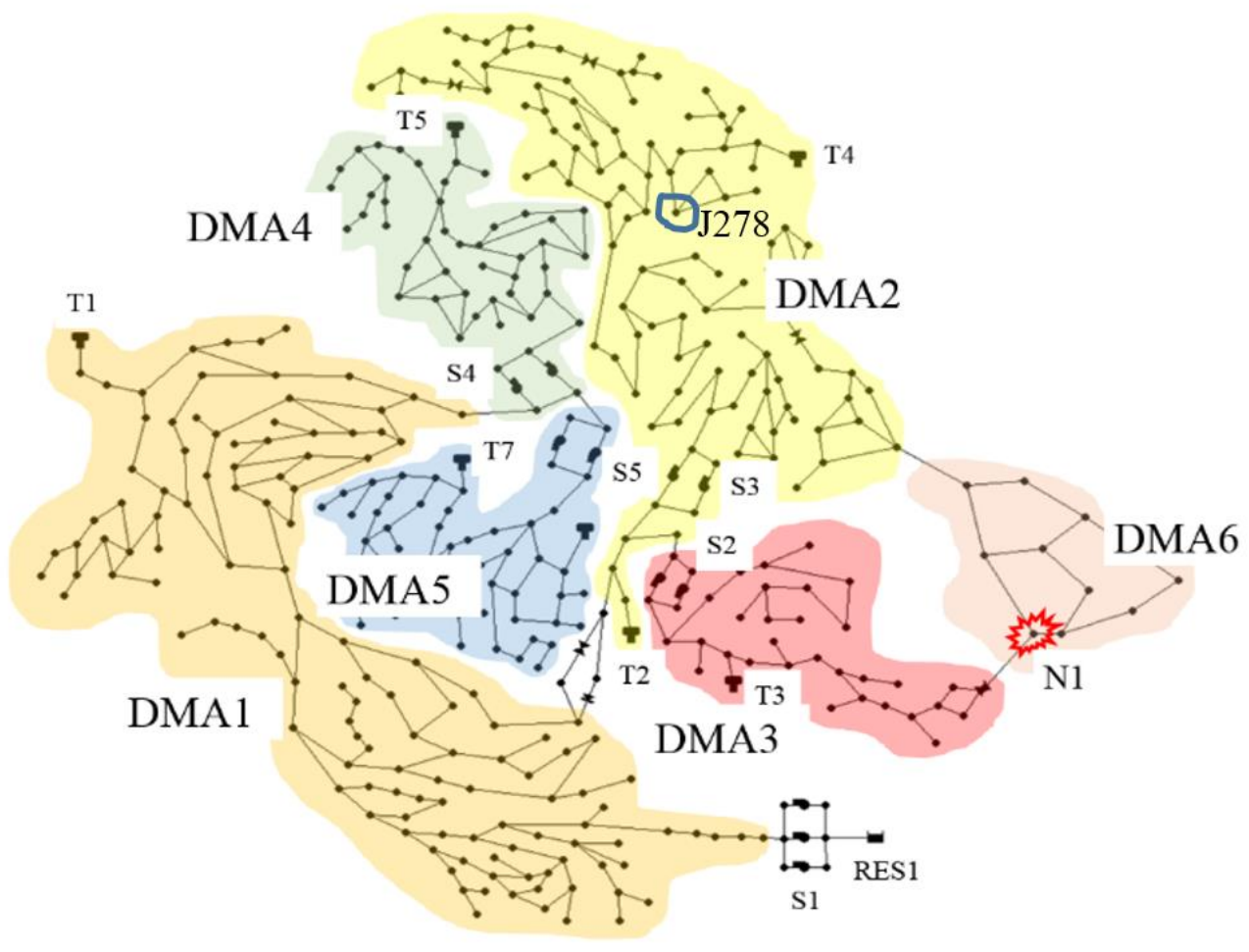

Figure 4.13. Network layout for case study 4 network. 


\subsubsection{Results and discussion}

\subsubsection{Normal flow scenario}

Different values for hydraulic time step are used, as shown in Figure 4.14, to investigate the computational time and the numerical convergence for the SIPDM approach under different EPS conditions. The demand pattern time step is fixed at one hour. Also, for comparison purpose, the artificial elements are connected selectively to only $60 \%$ of the network demand nodes for all hydraulic time steps during using SIPMD approach.

Both the EPANET2.0 and the SIPDM approaches yielded almost the same results in terms of nodal flows and pressures for all time steps. This can be clearly observed from the Figure 4.15. Where the difference in total water delivered (e.g., error) to the network using two approaches is very small and can be neglected (i.e., varied from 0.0115 to $0.0135 \mathrm{l} / \mathrm{s}$ ) for all hydraulic time steps considered. Figure 4.14 shows also that the computational times for both solvers are increasing from $0.7 \mathrm{~s}$ to about $2.5 \mathrm{~s}$ with decreasing hydraulic time steps. As expected, the SIPDM approach is slower that the EPANET2.0 solver in all cases, but not significantly. For example, for 60 s time step, SIPDM is $29 \%$ slower than EPANET2.0. This difference is acceptable and is due to the fact that in the SIPDM approach artificial elements are added to $60 \%$ of network demand nodes to model pressure-driven demand. Thus, the flow settings for FCV and emitter coefficient for all demand nodes are updated at the beginning of each pattern time steps (i.e., every hour here). While, in the EPANET2.0 solver, the demand values are only updated at demand nodes (i.e., every 2 hours here).

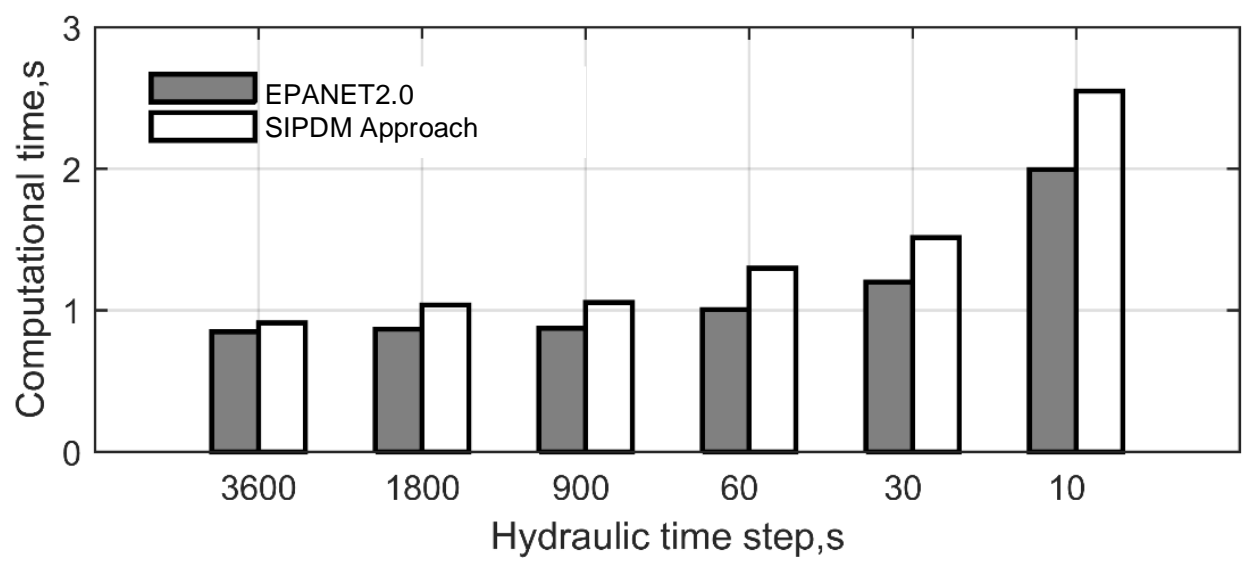

Figure 4.14. Average computational time comparison for EPS for the normal flow scenario for case 4 network. 


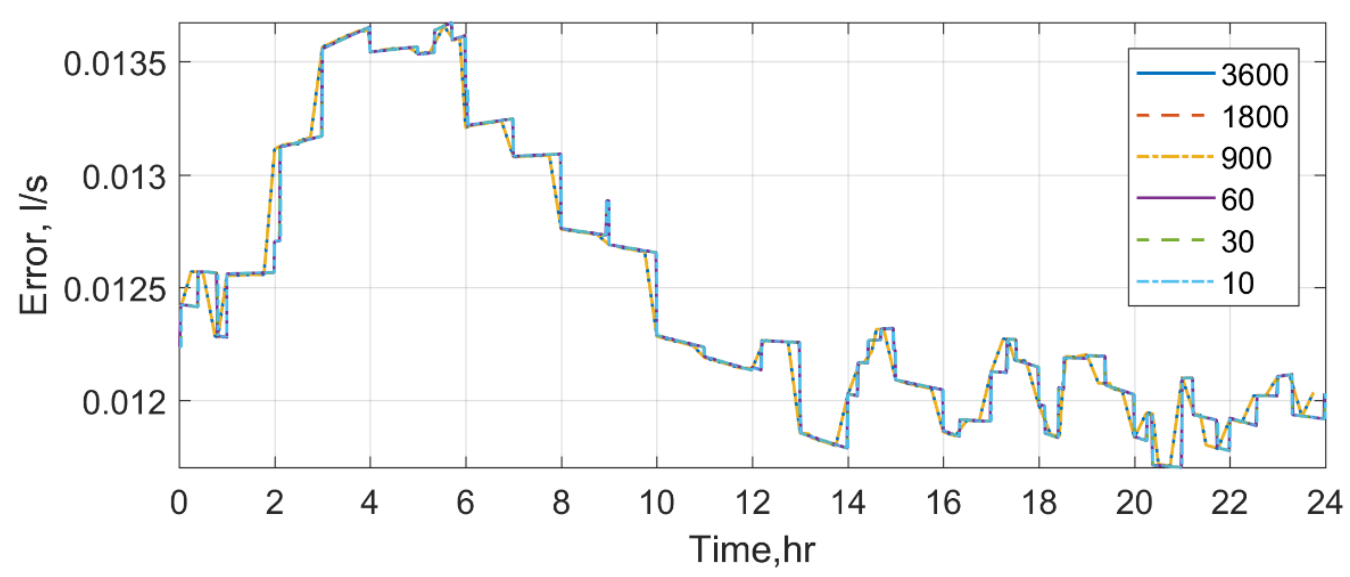

Figure 4.15. Errors observed in term of the total system flow between SIPDM and EPANET2.0 for the normal flow scenario for case 4 network.

\subsubsection{Abnormal flow scenario}

The abnormal flow scenario is created by subjecting node N1 in DMA6 (as shown in Figure 4.13) to an additional constant demand of $15 \mathrm{l} / \mathrm{s}$ from 12:00 to 20:45, thus simulating a large burst (or abnormal demand) at that location. Accordingly, the base demand at node $\mathrm{N} 1$ has changed from 0 to $15 \mathrm{l} / \mathrm{s}$ during this period, whilst the required demands for $\mathrm{N} 1$ are presented in the corresponding emitter connected to N1. The hydraulic time and pattern time steps were fixed at 15 minutes and one hour, respectively. Simulations by EPANET2.0 predicted pressure heads below $P_{\text {req }}$ at all 10 nodes with demands in DMA6 during the whole overloading condition period as shown in Figure 4.16. This number increased at 17:00 (the most severe time) to 26 nodes affected in DMA2. Because of these failure conditions, the proposed artificial elements are connected to all 36 deficient demand nodes for the whole simulation period considered. 


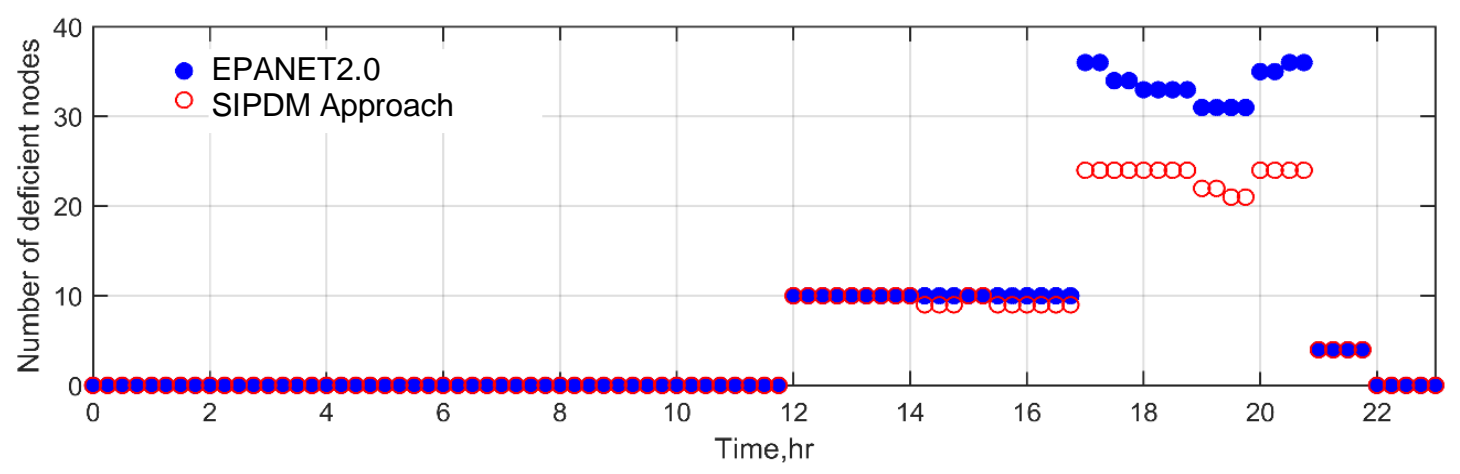

Figure 4.16. Number of nodal demands deficient under abnormal flow scenario in the case 4 network.

In comparison to EPANET2.0, the SIPDM approach predict less nodes with no or partial flow. Where, the SIPDM approach shows that only 24 nodes were affected by low pressure conditions at 17:00, as illustrated in Figure 4.16. This is because the water depth in tank T4 dropped to zero, as shown in Figure 4.17 and pumping stations S2 and S3 were unable to deliver the required demands to all nodes. The number of pressure deficient nodes decreased slightly in both solver solutions during low demand patterns. By 20:15 the number of pressure deficient nodes increased again in EPANET2.0 and the SIPDM approach to 36 and 24, respectively. Therefore, the proposed SIPDM method estimated less deficient nodes in comparison with EPANET2.0. This is because the SIPDM approach applied the NHFR function for each node individually, whilst EPANET2.0 satisfied demands at all nodes regardless of the available nodal pressure heads, which produced more nodes with pressure heads below $P_{\text {req }}$ or below $P_{\text {min. }}$. Both solvers predicted four pressure deficient nodes in DMA2 after the additional demand at $\mathrm{N} 1$ was removed (e.g., at 21:00). This is because the water depth in tank T4 is zero as compared to around $0.5 \mathrm{~m}$ during normal loading conditions (see Figure 4.17). By 22:00 the system was back to the normal pressure conditions.

Also, the obtained results reveal that the SIPDM approach converged to a numerically stable and deliver logical solution under the deficient pressure conditions and it was faster by approximately $5 \%$ under normal flow conditions. For example, Figure 4.18 shows the prediction of delivered demands and pressure heads at node $\mathrm{J} 278$ (the location of this typical node is shown in Figure 
4.13). As can be seen from this figure, both EPANET2.0 and SIPDM delivered identical flows at this location when the available pressures were sufficient to deliver the required demand. On the other hand, as expected, only a partial demand was delivered at this node in SIPDM when the pressure head went below the threshold required $\left(P_{\text {req }}\right)$.

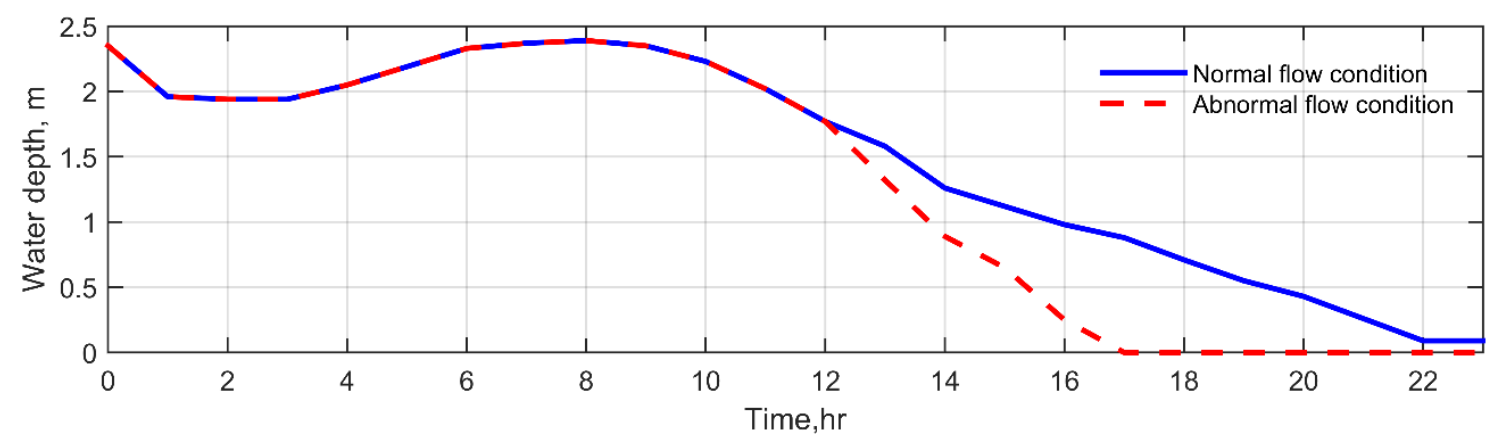

Figure 4.17. Water depth at tank T4 for case 4 network.

(a)

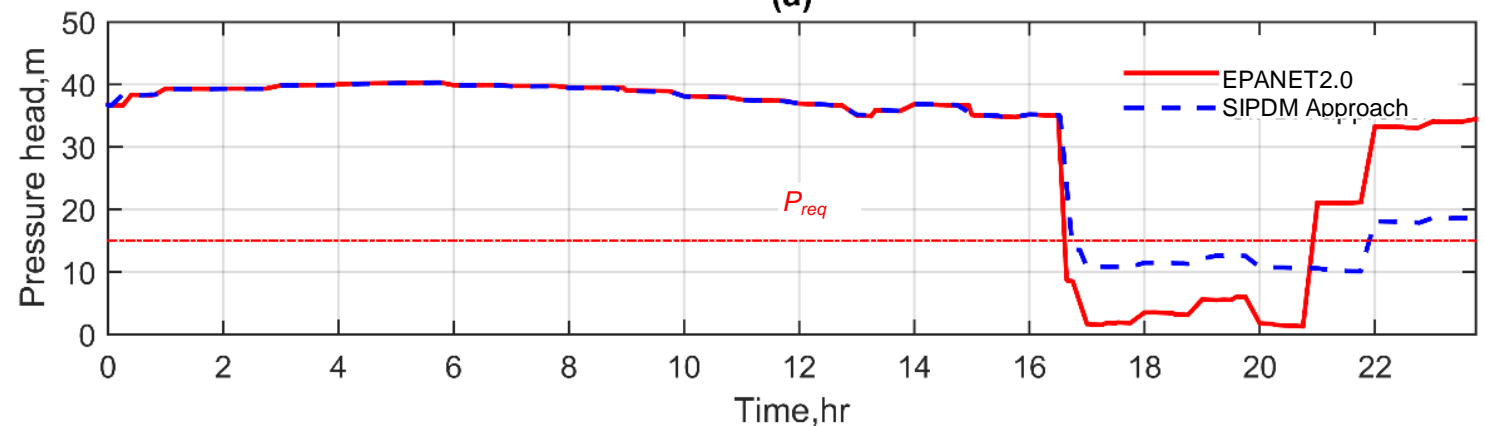

(b)

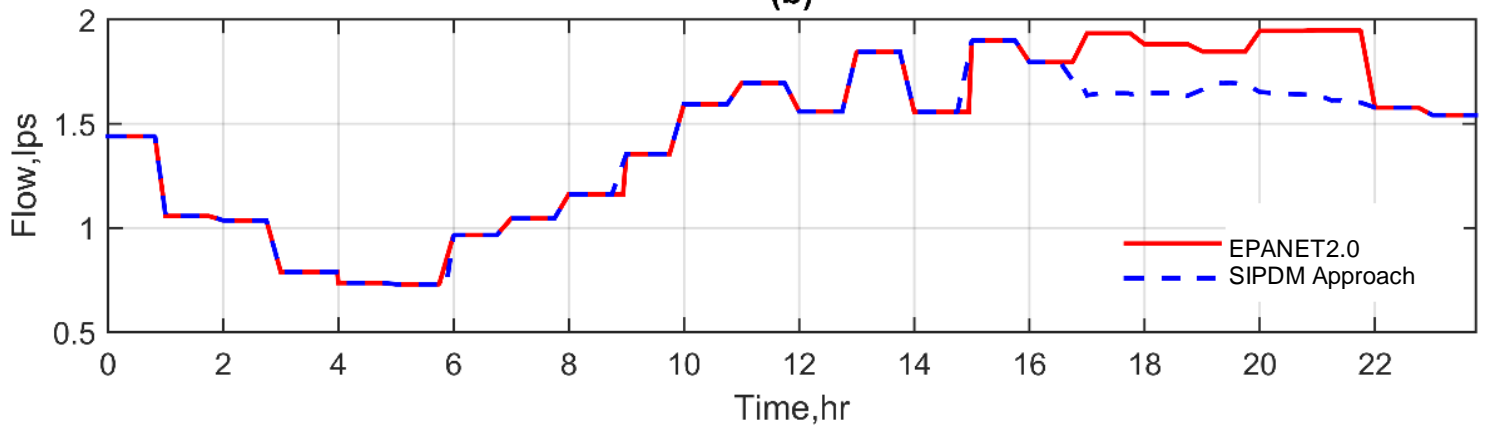

Figure 4.18. The prediction of (a) pressure heads and (b) flows at node J278 for case 4 network. 


\subsection{Summary}

The SIPDM approach presented in chapter 3 was validated, demonstrated, and compared to several other PDM methods reported in the literature on a number of benchmark and real-life networks in this chapter. In section 4.2, the SIPDM approach is applied on a simple looped network under steady-state analysis, and the obtained results were compared to some other PDM methods found in literature. Then, the approach is demonstrated and validated on a number of more complex, large and challenging networks under different flow conditions for steady-state analysis. In section 4.4, the validated SIPDM approach is applied on a simple network under EPS analysis. Finally, the approach was examined on a large semi real-life case study under EPS analysis.

The results obtained demonstrate the ability of the SIPDM approach to predict network pressures and flows in a consistently accurate, numerically stable, and computationally efficient manner under pressure-deficient and normal-flow conditions in both steady-state and EPS. Also, in comparison to some other common PDM methods found in literature as well as DDM method (i.e., EPANET2.0 solver), the SIPDM possess the following key advantages: (1) it predicts the same or more-accurate pressures and flows for network nodes under both pressure-sufficient and pressure-deficient conditions; (2) except EPANET2.0, it does this in a generally more computationally efficient manner, especially when applied to larger pipe networks as illustrated in case studies 2; and (3) unlike some PDM methods, it can be used for extended period simulation, i.e., not just steady-state hydraulic analysis.

In the next chapter, the full performance of the real-time operational response methodology presented in chapter 3 is tested, verified and demonstrated under two failure scenario on a real-life D-Town network. 


\section{CHAPTER 5: CASE STUDY ON REAL-TIME OPERATIONAL RESPONSE METHDOLOGY}

\subsection{Introduction}

This chapter illustrates the application of the real-time operational response methodology presented in chapter 3 on a case study of D-Town network.

The chapter is organized as follows. After this introduction, the description of the case study is introduced and given in section 5.2. Once this done, the details of the interventions considered in the analysed case study in this chapter are described in section 5.3. In section 5.4, the details of the demand data in the case study network are provided. Then, the description and the details of the two hypothetical failure event scenarios (i.e., pipe burst) are given in section 5.5. section 5.6 reports the results and discussions obtained under each failure event scenario in two separate sub-sections, also the obtained results are compared with the enumeration method for performance comparison purpose. Finally, the chapter is summarised and relevant conclusions are drawn in section 5.7.

\subsection{Description}

The proposed real-time response methodology in chapter 3 has been tested and verified on the modified D-Town water distribution network shown in Figure 5.1. D-Town network is based on a real-world medium-sized network. This benchmark network was introduced for the Battle of the Water Calibration Networks (BWCN) (Ostfeld et al. 2010) and subsequently used for the Battle of the Water Networks II (BWN-II) (Marchi et al. 2012). This system is chosen for its real-world complexity of having multiple tanks supplying water to multiple pressure zones, with the subsequent need to control multiple pump stations.

The D-Town network introduced at the BWN-II competition consists of five district metered areas (DMAs) (i.e., DMA1-DMA5) required to be upgraded and an additional new zone to be designed (i.e., DMA6) in order to cope the increased water demand. The Guilodin et al. (2012) solution was chosen by the BWN-II scientific committee as the best one (Marchi et al. 2013) and hence is used here 
as a case study to validate the real-time response methodology presented in chapter 3.

In total, the above D-Town network consists of 443 pipes, 399 nodes (of which 348 are demand nodes), 11 pumps, five pressure reducing valves (PRVs), one throttle control valve (TCV), 7 storage tanks, and a single reservoir with constant head. The network services approximately 240,000 inhabitants. The pipe network properties, and other pump, valve, and nodal data were taken from Marchi et al. (2012). The modifications made to the original network are shown in black in Figure 5.2 and their detail are summarized in Table 5.1. All data for the existing network components were incorporated into the EPANET2.0 (Rossman 2000a) input file $D$-Town.inp available and can be downloaded from the Centre for Water Systems at the University of Exeter (CWS 2018).

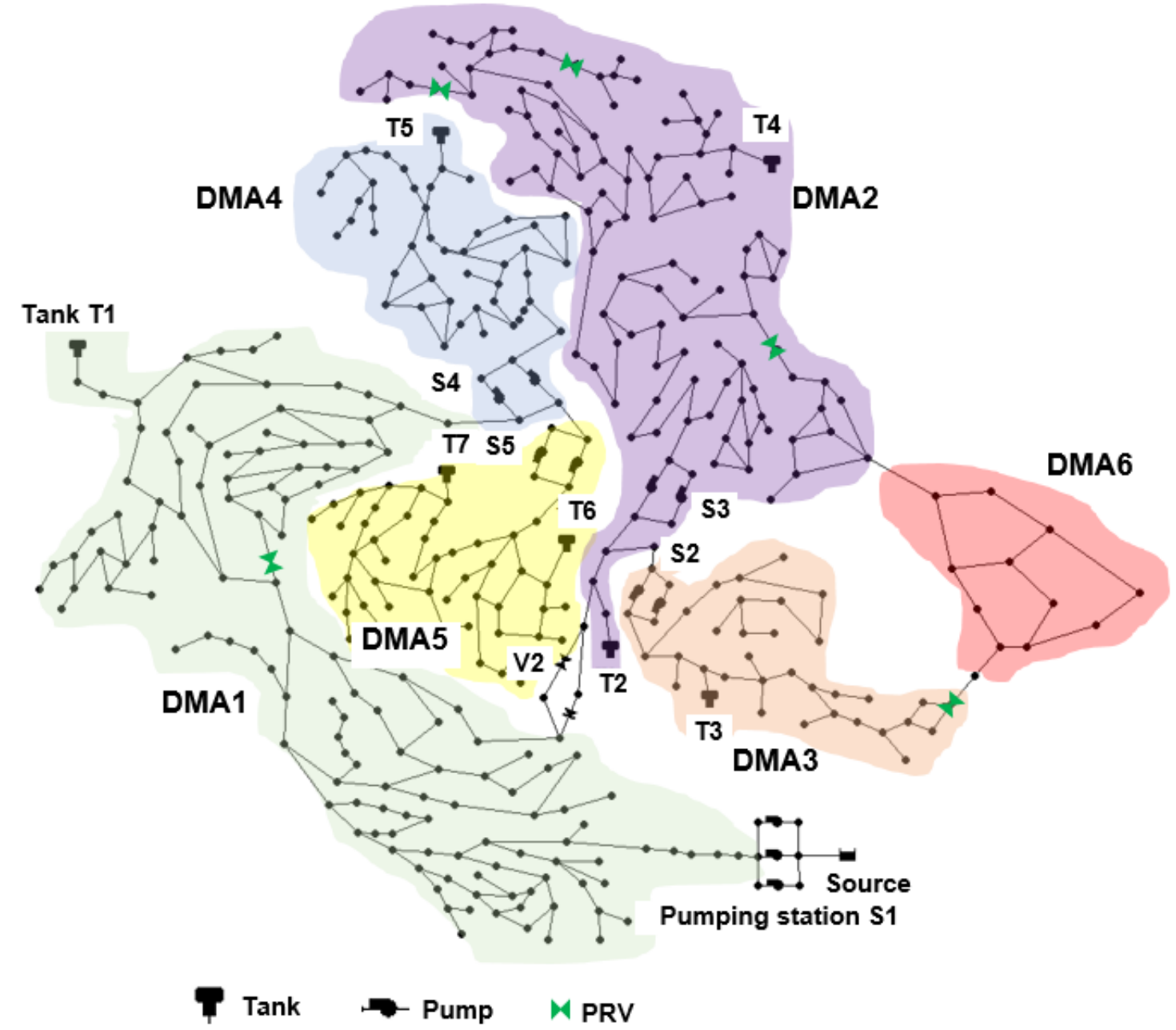

Figure 5.1. D-Town network layout (Marchi et al. 2012). 


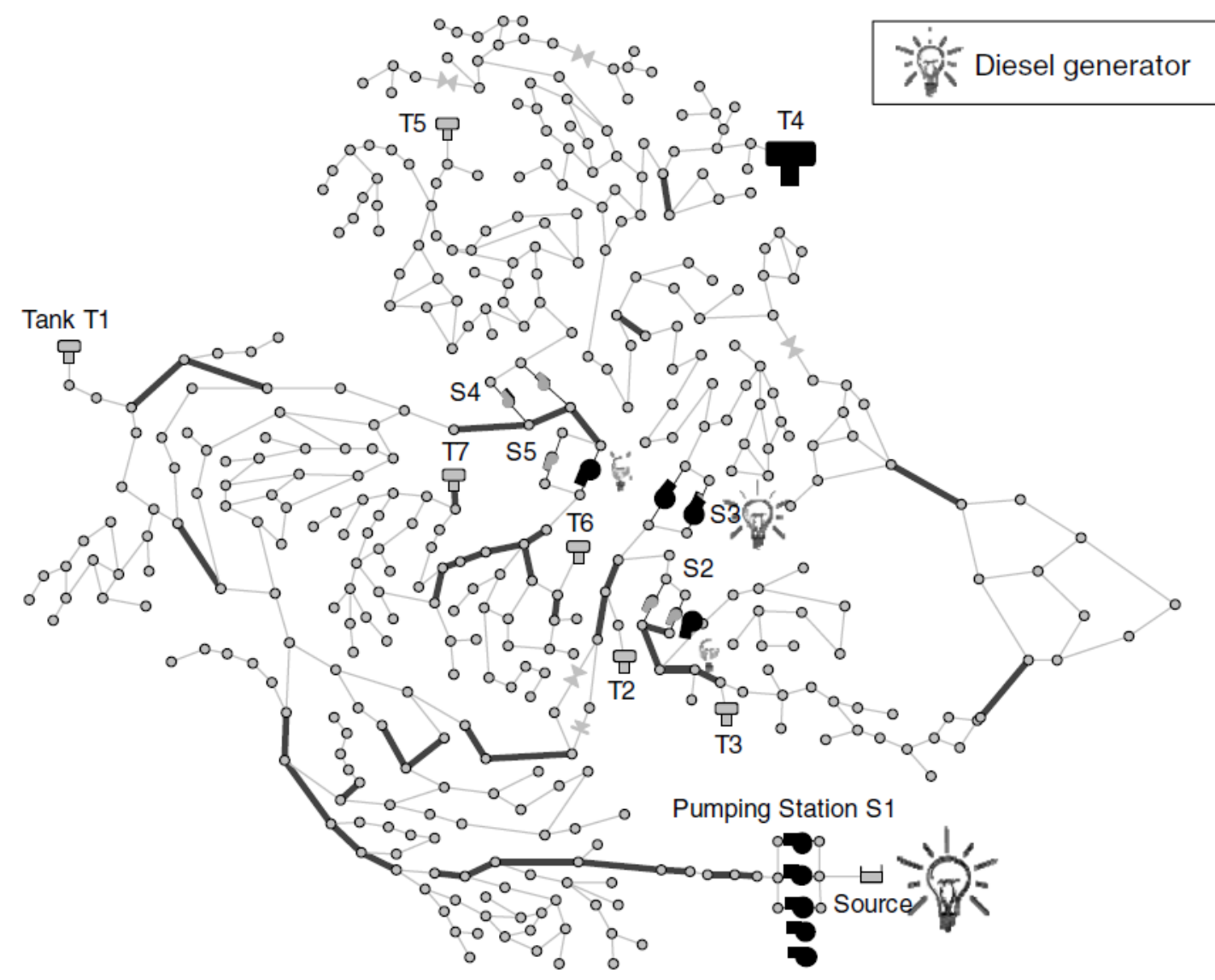

Figure 5.2. Schematic representation of the Guidolin et al. (2012) solution: modifications to the original network (Marchi et al. 2012) are shown in black.

Table 5.1 Modifications to the original D-Town (Marchi et al. 2012) by Guilodin et al. (2012)

\begin{tabular}{|l|l|l|l|l|}
\hline $\begin{array}{l}\text { Pump } \\
\text { replaced }\end{array}$ & $\begin{array}{l}\text { Pump } \\
\text { added }\end{array}$ & $\begin{array}{l}\text { Tank \# } \\
\text { (volume } \\
\left.\text { added, } \mathrm{m}^{3}\right)\end{array}$ & $\begin{array}{l}\text { Number of } \\
\text { replaced pipes }\end{array}$ & $\begin{array}{l}\text { Diesel } \\
\text { generator } \\
\text { inserted }\end{array}$ \\
\hline $\begin{array}{l}\text { PU1(10a), } \\
\text { PU2 (8b), } \\
\text { PU3 (10a), } \\
\text { PU6 (10a), } \\
\begin{array}{l}\text { PU7 (8b), } \\
\text { PU10 (10a) }\end{array}\end{array}$ & PU4-1 (10a & & T4 (1,000) & 5.2) \\
\hline
\end{tabular}

Note: the pump curve is reported in brackets in column one 


\subsubsection{System operation}

The D-Town system operation is shown on a schematic in Figure 5.3. The water is supplied to the town by a large reservoir (R1) and it is balanced via seven water tanks (T1-T7). Water is pumped into the system by using pumping station $\mathrm{S} 1$ and delivered to tanks T1 and T2. Water supply to T2 is controlled by the TCV (V2). Pumping stations S2 and S3 supply water to tanks T3 and T4 respectively, pumping station S4 supplies water to T5 and station S5 supplies water to tanks T6 and T7.

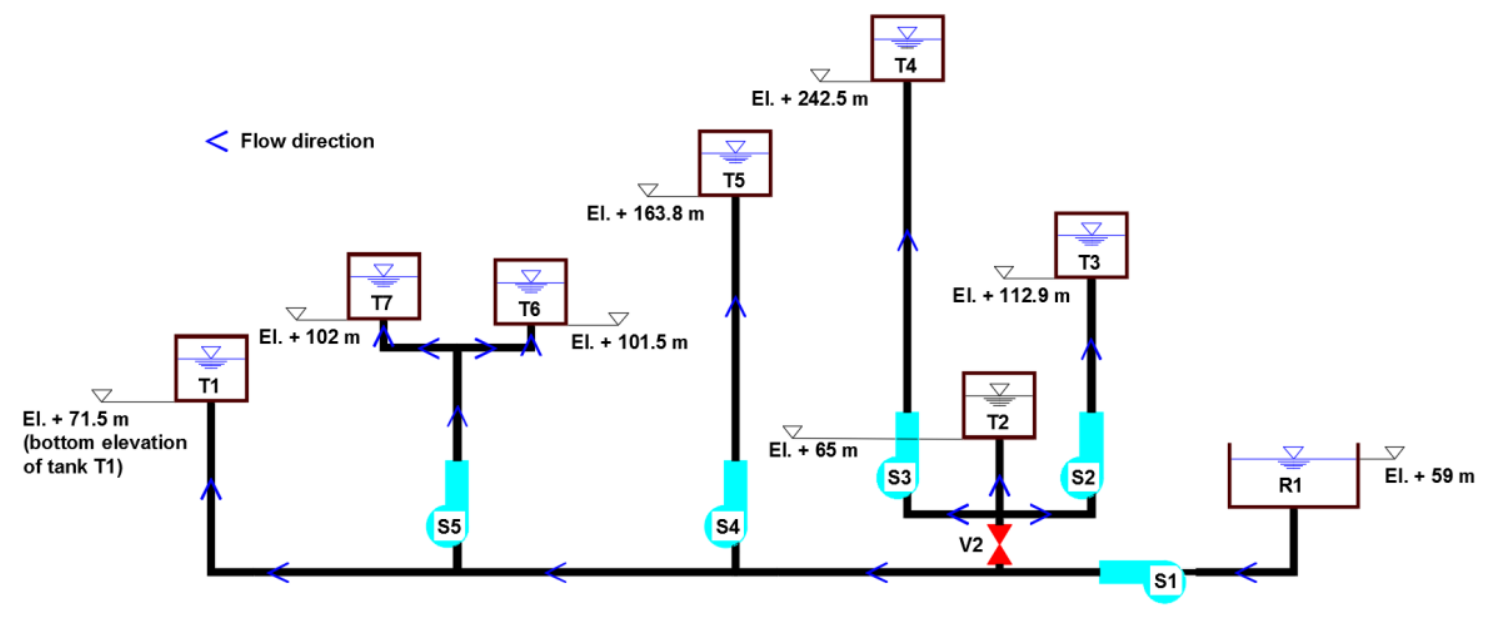

Figure 5.3. D-Town system schematic (after Ostfled et al. 2012).

The D-Town network was analysed under two operational scenarios, a normal operation scenario, for which the network was subject to normal demand loadings, and a power outage event scenario. The power outage was assumed begin at any hour within the analysed $168 \mathrm{hrs}$ (i.e. one week) simulation time, and to last for two hours. Within the power outage scenario, all pumps not powered by diesel generators are required to be shut down. In both scenarios, the system is able to provide a required pressure of $20 \mathrm{~m}$ or more to nodes with a non-zero demand and a minimum pressure value of zero to other zero-demand nodes. Also, the constraint of staying tanks to not be empty were met.

Figure 5.4 provides a typical water depth at each network storage tank (Guilodin et al. 2012) under normal demand loading scenario over $168 \mathrm{hrs} \mathrm{simulation} \mathrm{time.}$ the initial volume at each tank over the analysed simulation period was set to the half of the volume of the tank in order to ensure that tanks are not stayed empty 
during the normal operation analysis period. Each tank is described by using the following 5 values: diameter, elevation, minimum level, maximum level and initial level as illustrated in Figure 5.5. Under normal operation scenario, the tank water level is forced to stay between the minimum and the maximum tank level, so that the volume between the tank bottom elevation and the minimum level represents a reserve volume. However, during power outage scenario, tanks are allowed to operate below their minimum level, i.e., to utilise the reserve volume. It is worth to mention that the minimum water level at tanks T1, T2, T4, T5 and T6 was set to zero. Whilst it set to $0.57 \mathrm{~m}$ at tank T3, and $0.09 \mathrm{~m}$ at tank T7. The power outage is allowed to occur at any time during the simulation.

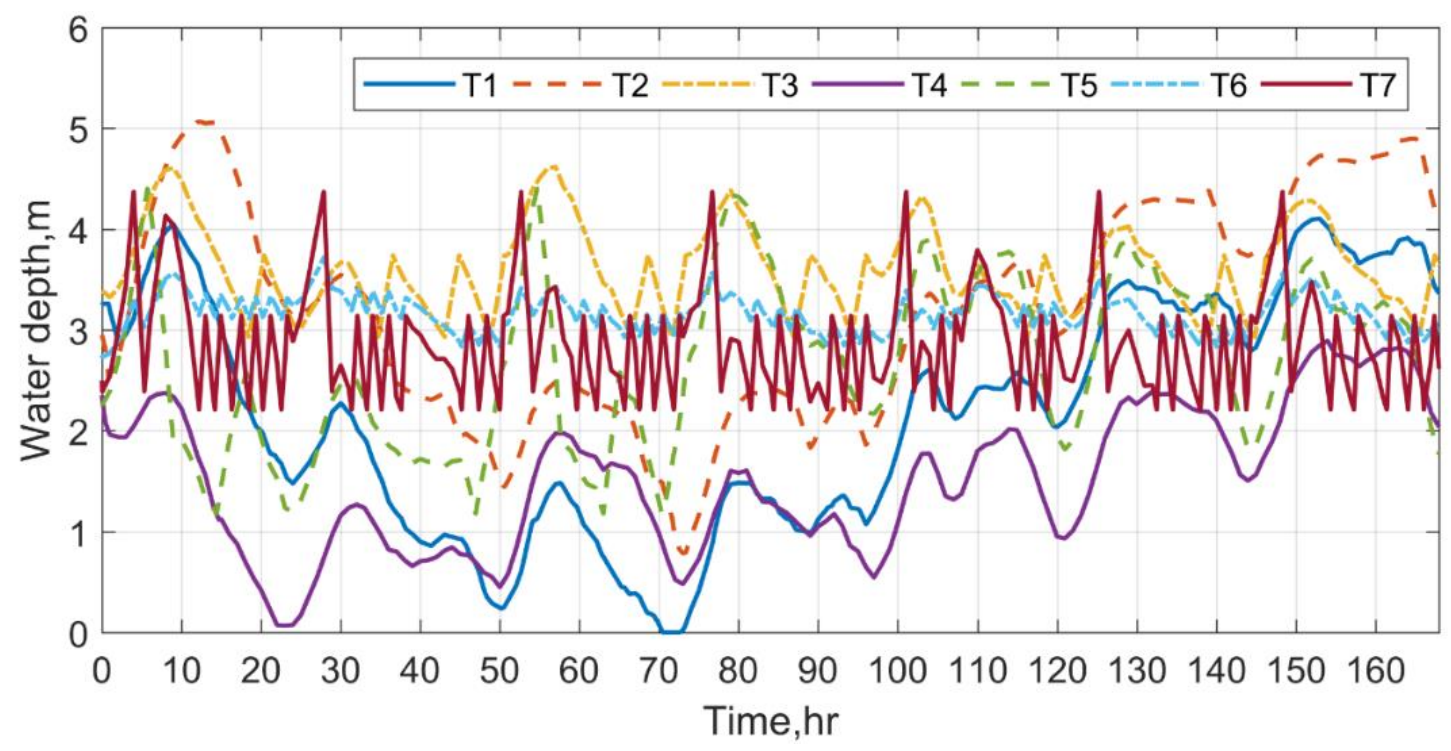

Figure 5.4. Water depth observed at the D-Town network tanks under normal system operation. 


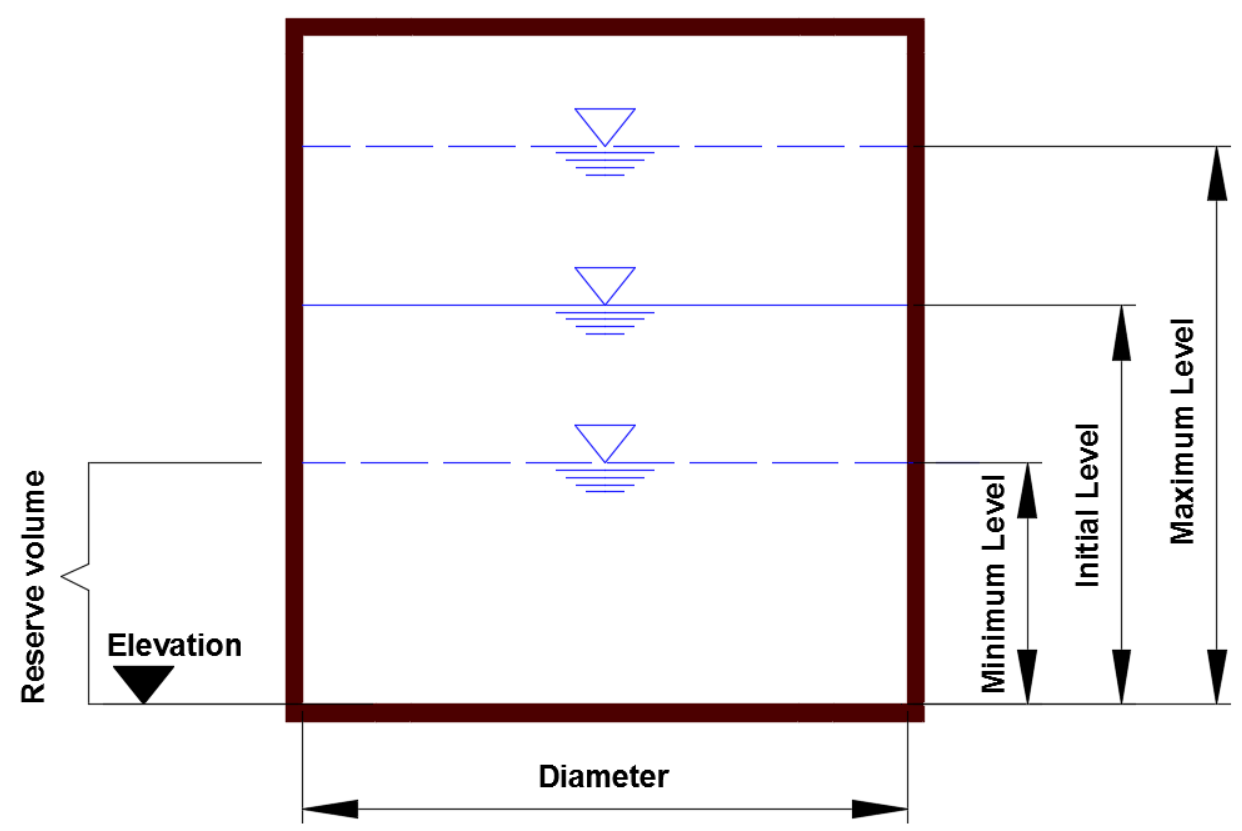

Figure 5.5. Tank description in EPANET2.0.

The operational response methodology shown in chapter 3 is tested on different failure events (e.g., pipe bursts) which are allowed to occur at any time during the day. Also, for the pipe failure starting at time $t_{0}$, initial water level in the tanks will be the one determined from the normal operation conditions at this time (see Figure 5.4). It is assumed that these tank levels would be available in a real-life system via the SCADA system. Additionally, an assumption is made here that tank's reserve volume is allowed to be used during failure events, i.e., starting from time to.

\subsection{Interventions}

The following interventions are considered in the real-time operational response methodology for the D-Town network.

\subsubsection{Isolation valves}

The information about the isolation valve locations is not available in D-Town. Therefore, 213 isolation valves were added to the network as shown in Figure 5.6. The locations of these valves were obtained based on the guidelines given 
in Jun (2005). Namely, the configuration uses the $\mathrm{N}-1$ rule (Walski, 1993) as the starting point and a few valves are removed. Clearly out of 442 isolation valves, 229 are removed. The removed valves usually tend to be those placed on the upstream pipe (i.e., the one where water most commonly flows to the node). All isolation valves in the network are assumed accessible, operational and initially fully open.

\subsubsection{Overland bypasses}

Due to the lack of real data, assumptions are made about the location of fire hydrants. A total of 133 network nodes are considered as hydrant points, as shown in Figure 5.6. The configuration of these hydrant points are obtained based on the hydrant installation guidelines given in Thames Water (2015). All fire hydrants in the network are assumed accessible and fully operational. The pairs of hydrants with connection distance $L D \leq L D_{\operatorname{Max}}=300 \mathrm{~m}$ are considered as potential overland bypass candidates. This $L D_{\operatorname{Max}}$ value was chosen in consultation with engineers in a UK water company. Accordingly, a total of 307 possible overland bypasses were identified. The overland bypasses are assumed to have a nominal diameter of $200 \mathrm{~mm}$ and Hazen-Williams roughness coefficient of 100. The possible overland bypasses are modelled as additional (dummy) network pipes in the EPANET2.0 input data file and hydraulic solver with initially closed status.

\subsubsection{Pressure reducing valves (PRVs)}

There are five PRVs in the D-Town, namely V45, V47, V1, N15 and V40, and their locations are shown in Figure 5.6. These PRVs can be used as potential recovery interventions in the proposed response methodology by modifying the regular PRV settings. The size (i.e., diameter) and regular pressure settings for each PRV are described in Table 5.2. The PRV setting is allowed to change at the optimization stage as follows: no increase, $5 \%$ increase, $10 \%$ increase, $15 \%$ increase, $20 \%$ increase or $25 \%$ increase, all relative to the regular pressure setting. 
Table 5.2. Properties for the PRVs in the D-Town network.

\begin{tabular}{|c|c|c|}
\hline PRV identifier & Diameter, $\mathrm{mm}$ & Regular pressure setting, $\mathrm{m}$ \\
\hline V45 & 152 & 30.8 \\
\hline V47 & 102 & 26.73 \\
\hline V1 & 203 & 37.11 \\
\hline N15 & 102 & 0 \\
\hline V40 & 203 & 50 \\
\hline
\end{tabular}

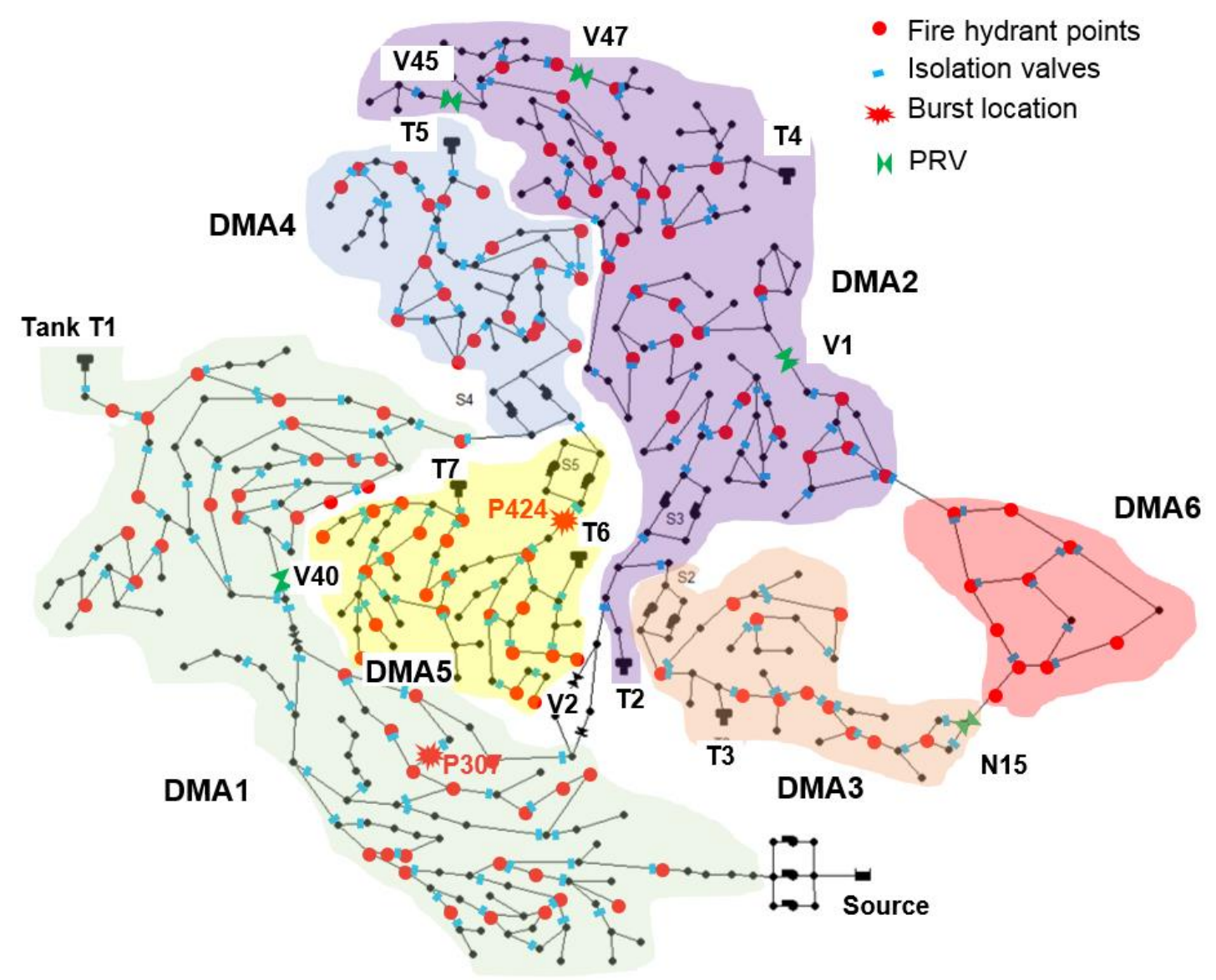

Figure 5.6. The location of pipe bursts, isolation valves, hydrant points, and PRVs in D-Town network. 


\subsection{Demand Data}

Monthly water demands taken from the billing records at each node are available for a period of $168 \mathrm{~h}$ (1 week). The typical diurnal patterns for each DMA are estimated from the above real demand data and their values are illustrated in Figure 5.7. These patterns are used to estimate the required demand at each network node at any time in the duration $168 \mathrm{hr}$ by multiplying the base demand and corresponding multiplier value shown in Figure 5.7. The patterns are a composite of demands that occurs during weekday flows, weekend flows and seasonal flows. Due to varying diurnal patterns in each DMA, several time periods were found as peak conditions, for example, 166:00 hr (maximum demand for whole system), 117:00 hr (maximum demand for DMA 5), 105:00 hr (maximum demand for DMA 3 and 6), 53:00 hr (minimum demand for the whole system) and $3 \mathrm{hr}$ (minimum demand for DMA 5).

Changes in consumer water demands behaviour during the burst event are not considered in the case study presented in this chapter, and, instead, consumer demands are simulated as homogeneous, and aggregated only at nodes using the flow patterns shown in Figure 5.7. All nodes are assumed to be residential consumers. The number of consumers at each residential node is calculated by dividing the base demand value at that node by the daily water consumption per consumer, which is assumed as 150 litres per person per day (Aquaterra 2008). Figure 5.8 shows the based demand and the number of consumers allocated to each residential node. 


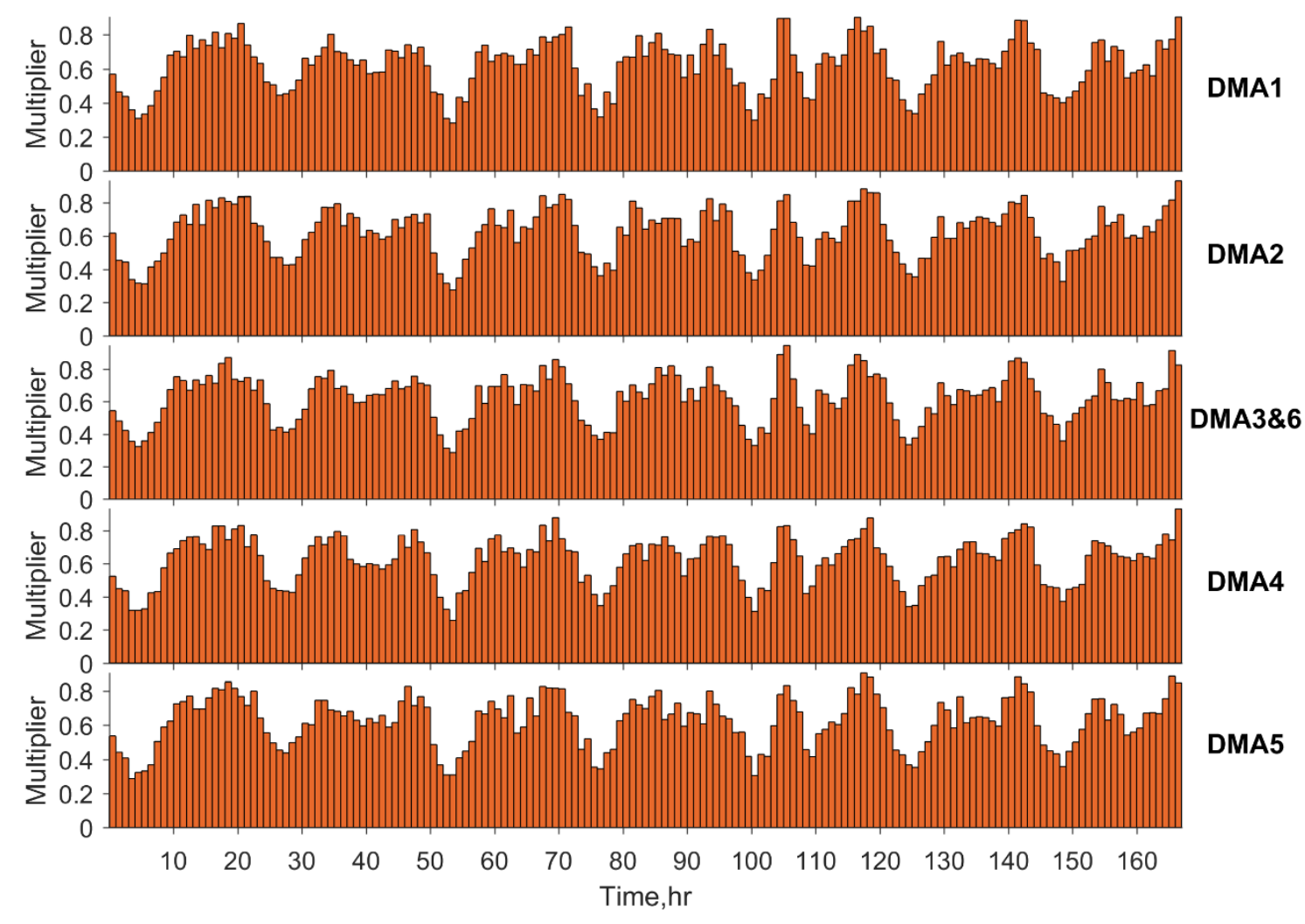

Figure 5.7. Demand multiplier factors for D-Town network.

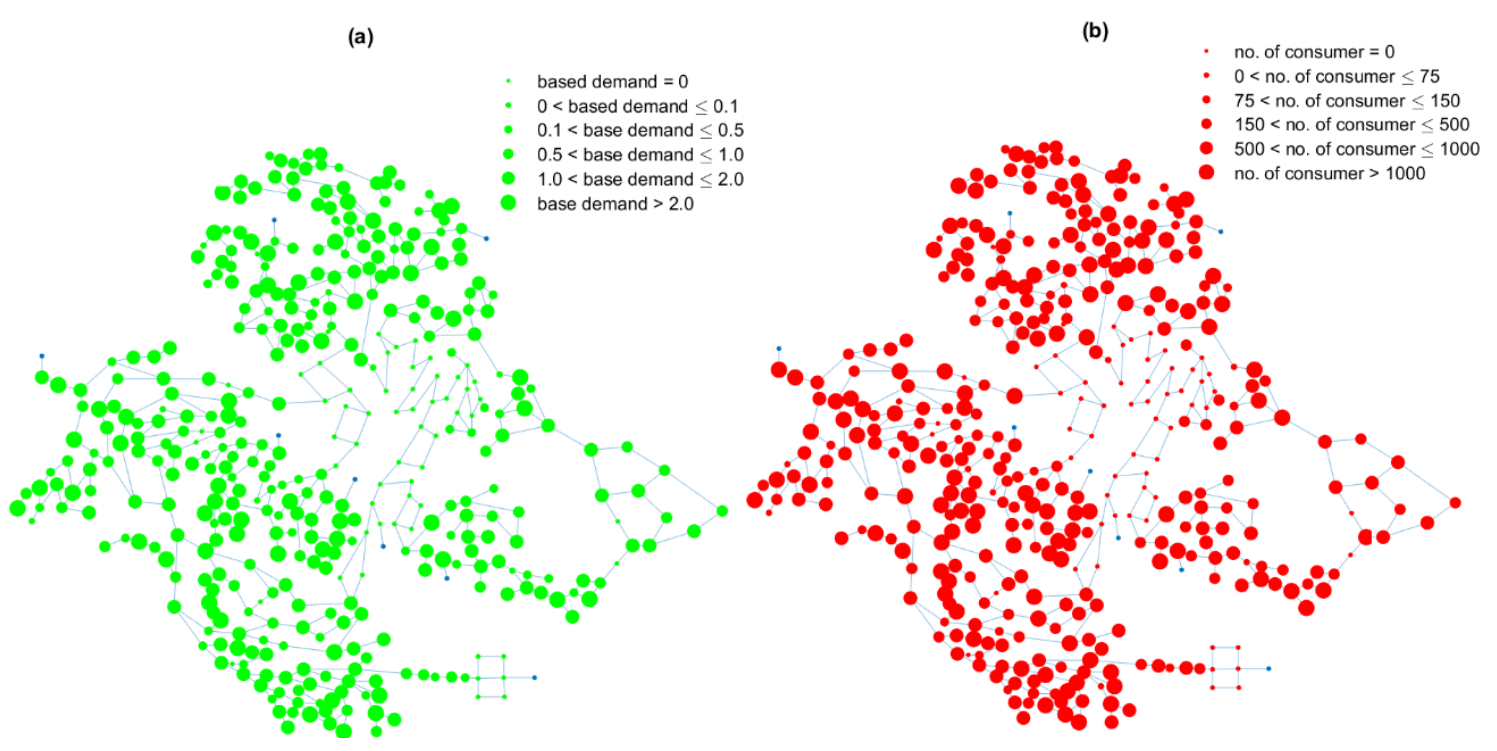

Figure 5.8. Allocated (a) base demand (b) number of consumers at each node for D-Town network. 


\subsection{Failure Event Scenarios}

The network and response methodology are tested and demonstrated under two hypothetical pipe failure event scenarios. The first scenario involves a large burst on pipe P307 at an off-peak hours in DMA1 with a variable outflow having an average value of $11.2 \mathrm{l} / \mathrm{s}$ as shown in Figure 5.9. The second scenario involves another medium burst on pipe P424 in DMA5 that occurs at the peak demand hour with a variable outflow, having an average value of $6.4 \mathrm{l} / \mathrm{s}$ as shown in Figure 5.9. Both pipes are critical in terms of water supply and their locations are shown in Figure 5.6. Pipe P307 is one of the key pipes in the network supplying water to the bounded area by the hidden line of DMA1, and DMAs 2, 3 and 6, while P424 is supplying water to DMA 5 as shown in Figure 5.6.

The first scenario has been selected to demonstrate the performance of the response methodology in term of search space reduction whilst finding optimal recovery interventions within the affected DMAs and adjacent DMAs in near realtime. The second scenario aims to demonstrate the effect of bringing water into an affected area by changing the valve settings and installing overland bypasses in a nearby unaffected DMA1 with the aim to enhance flow conditions (i.e., supply interruption, pressure) in a disconnected DMA5 in near real-time.

The results obtained from both scenarios are also compared to the results obtained by using the Enumeration method with the aim of assessing the overall performance and convergence of the NSGA-II that used at the optimization stage in the proposed response methodology in chapter 3.

Pipe bursts for the two scenarios can be modelled in a number of ways, as discussed in the literature review chapter in section 2.3.2. The realistic way chosen in this chapter models bursts using EPANET's emitters as pressure dependent outflows with the simplifying assumption that their pressure exponent is always equal to 0.5 , i.e. regardless of pipe material or other factors. Therefore, to simulate pipe bursts on pipes P307 and P424, pressure dependent outflows are assigned to the downstream nodes (Yoo et al. 2016) of respective pipes as follows:

$$
Q=C_{d} \cdot P^{r}
$$


Where: $Q$ is the flow rate, $P$ is pressure at downstream node, $C_{d}$ is a discharge coefficient and $\gamma$ is a pressure exponent. The above generalized orifice equation is used in EPANET for modelling the pressure sensitive outflow such as pipe bursts and leakage.

As mentioned above, the $y$ exponent value is fixed at 0.5 . The value of this exponent can generally vary from 0.5 to 2.5 , however, as suggested by Cassa et al. (2010) values higher than 1.5 estimated during field trials do not have grounds in theoretical foundations. The chosen value of the emitter exponent of 0.5 agrees with Lambert (2002) who suggested that detectable leaks and bursts in metal pipes typically have values of exponent close to 0.5 . In this case, the emitter coefficient is calculated based on the estimated burst flow and actual pressure at the burst location. This approach provides results that are more realistic, however, it requires an extra steady state simulation to obtain pressure at the beginning of the impact simulation to calculate the value of the discharge coefficient $C_{d}$ using Equation 5.1.

The above two bursts are modelled using EPANET 2.0 emitters with discharge coefficient of 1.5 for P307 and 1.0 for P424. Figure 5.9 shows the outflow profiles from a pressure sensitive burst at pipe P307 and P424, from the time when burst occurred to the time of isolation, respectively. Note, the emitter coefficient is removed from burst pipe from the moment when the isolation starts, namely at 2:00 for the first scenario and 14:00 for the second scenario.

(a)

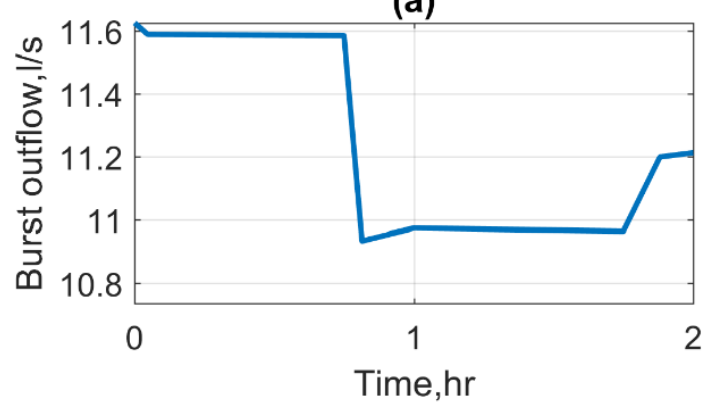

(b)

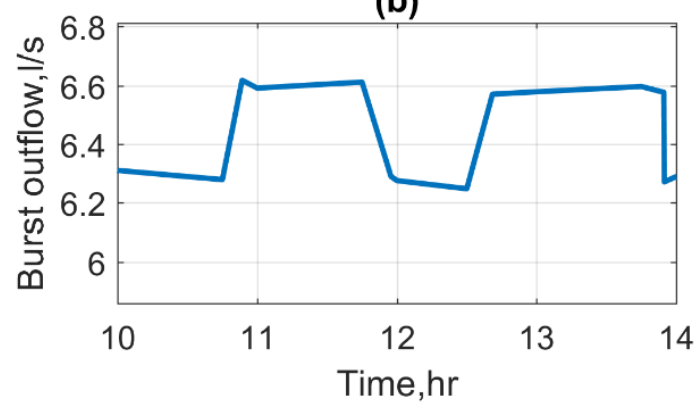

Figure 5.9. Outflow profile from the pressure sensitive burst at (a) pipe P307 (b) pipe P424. 
The hydraulic simulations are conducted using the EPANET 2.0 (Rossman 2000) solver based on the SIPDM method explained in the section 3.5.1 in chapter 3 . The full response methodology including optimization tool that explained in the flowchart given in Figure 3.6 in chapter 3 was developed and coded in the MATLAB R2015 environment. All simulation runs were performed on computer with the Intel(R) processor, Core (TM) i5-4570 CPU @ $3.2 \mathrm{GHz}$ and 64-bit Windows 7.

The following assumptions are made:

1. The burst in the first scenario is assumed to occur at 0:00, detected and located at 1:00 and ultimately isolated at 2:00. The pipe burst in the second scenario is assumed to occur at 10:00, detected and located at 13:00 and fully isolated at 14:00. Both bursts are assumed to remain isolated for 24:00 hours.

2. The impact assessment horizon for assessing interventions performance is assumed to be 24:00 starting from time 2:00 and last at time 26:00 for the first scenario, while it is assumed to start at time14:00 and last at time 38:00 for the second scenario.

3. Due to the lack of real cost data for interventions, the values of unit weight coefficients shown in Equation 3.3 in chapter 3 are assumed all equal to one (i.e., $w_{v}=w_{o}=w_{p}=1$ ).

4. It is assumed that no water is delivered to the consumers allocated to a node if the available pressure head is less than $0 \mathrm{~m}$, the demand is partially delivered if it is between 0-20 m and fully delivered otherwise. The emitter exponent for all added artificial emitters to demand nodes in the SIPMD hydraulic solver is assumed to be equal to 0.5 .

5. For the NSGA-II parameters crossover operator, mutation operator and population size, the following values were selected: $0.85,0.05$ and 20, respectively. 
6. The maximum allowed number of interventions $I N V_{\max }$ is limited to 10 (as explained in Chapter 3 section 3.4.3).

7. The hydraulic analysis in EPANT2.0 is assumed to be started at time 0:00 and last at time 26:00 for the first scenario, whilst it is assumed to be started at time 10:00 and last at time 38:00 for the second scenario. In both scenarios, hydraulic time step in EPANET2.0 is fixed at $15 \mathrm{~min}$ for all hydraulic runs.

\subsection{Results and Discussions}

\subsubsection{Segment identification analysis}

The first phase in performing the response methodology is to identify the system segments (offline) that can be used to isolate the failure location (online). The segment identification algorithm presented in section 3.3 is used here to define the segments in the D-Town network. A total of 152 of network segments were identified this way. Table A1 in Appendix A presents the summary of these segments. Out of the 152 regular segments, 36 segments result in unintended isolations. These are the segments that are disconnected from the source of DTown network and all storage tanks (i.e., T1 to T7), i.e., have no means of receiving water directly from any of these.

On average, each regular segment includes 3 pipes and 3 nodes, which need at least 3 isolation valves to separate this segment from the rest of the network. The largest segment identified contains 13 pipes, 12 nodes and requires closing 7 valves to isolate it. Whilst, the smallest segment involves only one pipe, one node and needs one valve to be closed to separate this segment from the rest of the network. Most of the smallest segments are those that contain a storage tank and the nodes which are normally located in at the dead-end pipes in the network.

The isolation of pipe P307 in the first failure scenario leads to the interruption of supply to four additional pipes (i.e., P302, P296, P120, and P118) and two nodal demands (i.e., J432, J433), as shown in Figure 5.10. The isolation of this segment requires closing 4 isolation valves located on pipes P120, P307, P302 and P118 (defined using green rectangular in the figure) and these are closed from 2:00 until 26:00. As a result of isolating the above segment, two hydrants (defined 
yellow circle in the figure) are disconnected from the rest of the network and they are dropped from the list of potential interventions during the recovery stage.

On the other hand, the isolation of a burst on pipe P424 in the second scenario led also to the disconnect pipe P53 and a non-demand node (J384) from the rest of the network, as shown in Figure 5.10. The isolation of P424 requires closing two isolation valves located on pipes P424 and P53 from time 14:00 to time 38:00.

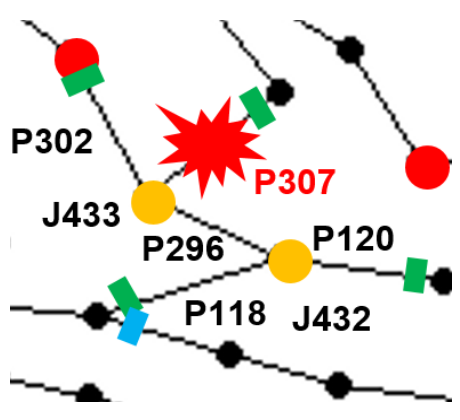

(a)

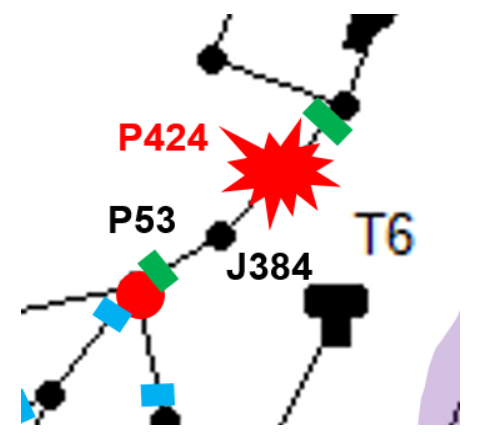

(b)

Hydrant point $\bigcirc$ Isolated hydrant point
Isolation valve $\quad$ Target isolation valve

Figure 5.10. Segmentation details for failure at pipes: (a) P307 and (b) P424.

\subsubsection{Response in the first failure scenario}

\subsubsection{Impact assessment}

The impact assessment of the simulated burst on pipe P307 on the system's ability to deliver requested water demand is shown in Figures 5.11. This figure shows the impact in terms of shortfall in the system. The shortfall is estimated by finding the difference between the total required demand (including the required demand at the isolated consumers) and the actual water delivered. The shortfall is calculated by using the SIPDM solver. Figure 5.11 also shows the total cumulative volume of water undelivered in the system (including the isolated area), which is estimated by using Equation 3.15. 
(a)

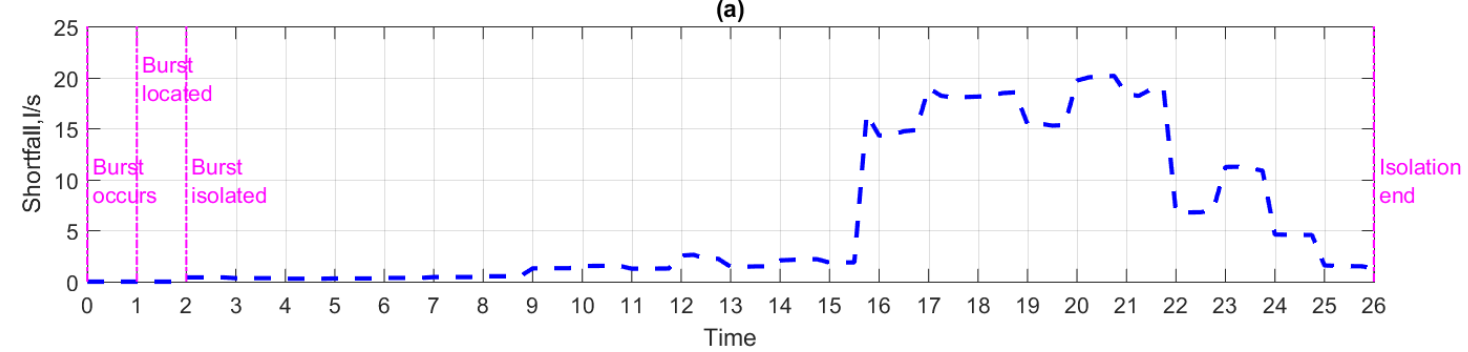

(b)

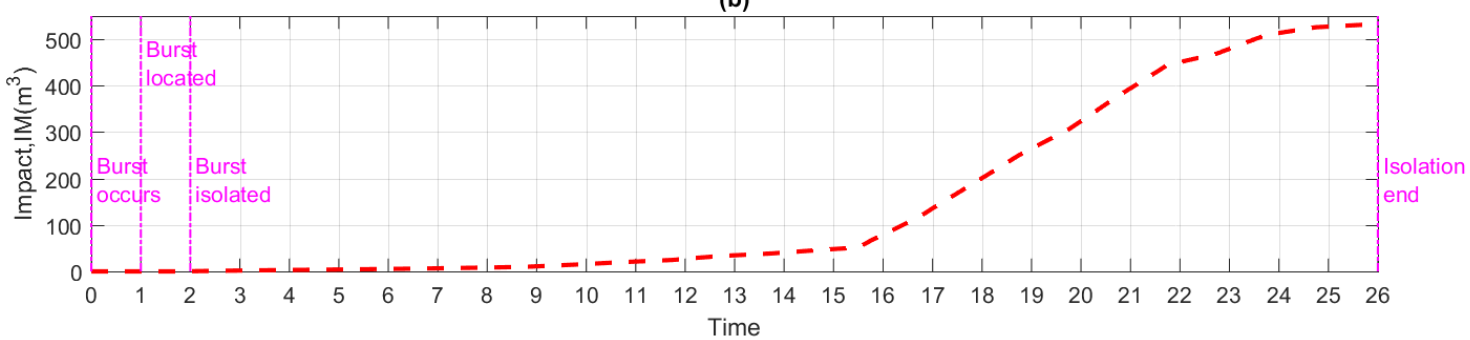

Figure 5.11. The estimated impact of pipe P307 failure on the system (including the isolated area) in terms of (a) shortfall (b) total cumulative volume of water undelivered.

The above impact assessment shows that nodal demands located downstream of the isolated area in DMA1 start to receive partial flow only 7 hours after the isolation (i.e., at 9:00), as illustrated in Figure 5.11. As mentioned earlier, the isolation of pipe P307 leads to the interruption of supply to four other pipes and two nodal demands from the main water supply in the network. As it can be seen from the Figure 5.11, the total cumulative volume of undelivered water to consumers increases steadily until about 16:00 when it suddenly starts increasing more rapidly. This is because at this point in time most nodal demands in DMA1 and DMA2 are starting to suffer from low-pressure conditions, This is due to the increasing demand requirement in the network and water in storage tanks T2 and T4 declining substantially and eventually draining out to zero at 13:00 and 16:00, respectively, as illustrated in Figure 5.12.

The major increases in undelivered demands occur at peak time after the isolation period (i.e., at 17:00, 20:00, and 22:00) as shown in Figure 5.11. On the other hand, all network nodes (except the isolated ones) remain less affected by pressure deficient conditions during the network low flow conditions after 22:00. Although the nodes located in DMAs 3 and 6 have partial water supply (prior to the isolation) through the pipes in the isolated area, they remain unaffected and 
have received all required demand over the whole impact assessment horizon. This is because the demand requirement (as shown in Figure 5.8) and elevation at these nodes are low compared to the rest of the network, and hence pressure heads are sufficiently high to deliver the full demand required. By the end of impact horizon (i.e., 26:00), the total of $533 \mathrm{~m}^{3}$ of water was not delivered as shown in Figure 5.11. This value is used as a reference $\left(I M_{R}\right)$ for evaluating operational recovery interventions.

Figure 5.13 shows also that the amount of water not delivered to the isolated area and the shortfall are relatively low when compared to total undelivered flow (IM) and shortfall in the system. It is worth to mention that the shortfall in the system that occurred between time 2:00 and 9:00 is occurring only because of the isolated consumers assigned to nodes $\mathrm{J} 432$ and $\mathrm{J} 433$, other demand nodes remain unaffected by the pipe burst. At the same time, the water depth at tank T2 and T4 declined compared to the normal operation scenario, as shown in Figures 5.12 and 5.4 .

(a)

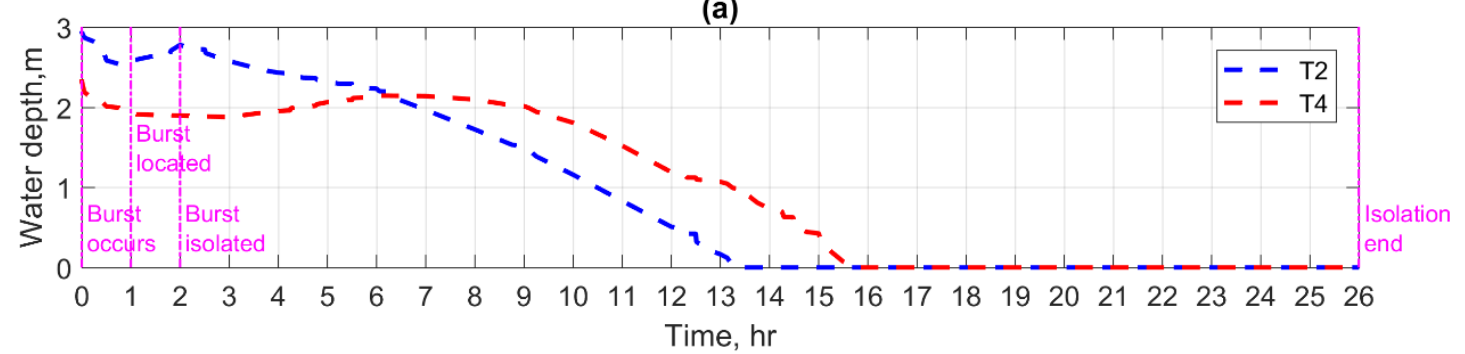

(b)

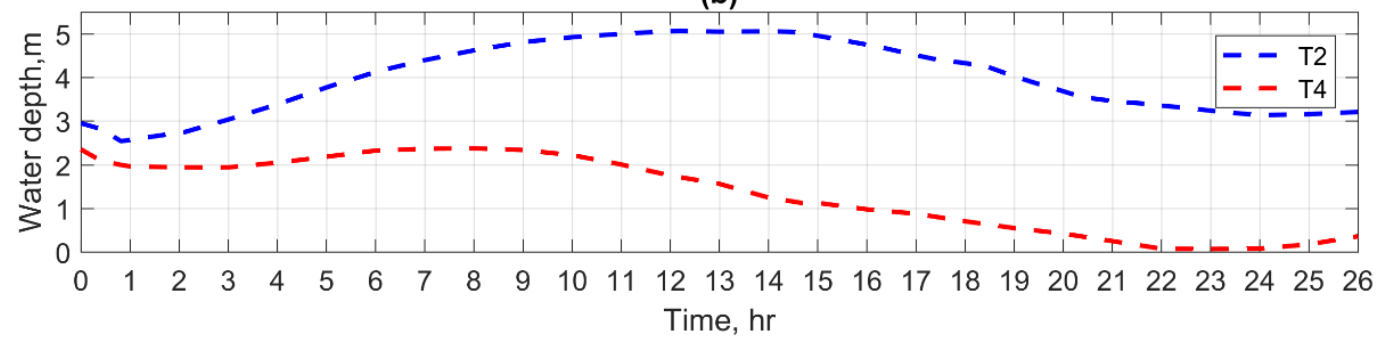

Figure 5.12. Water depth at tank T2 and T4 under (a) pipe P307 failure scenario (b) normal operation scenario. 

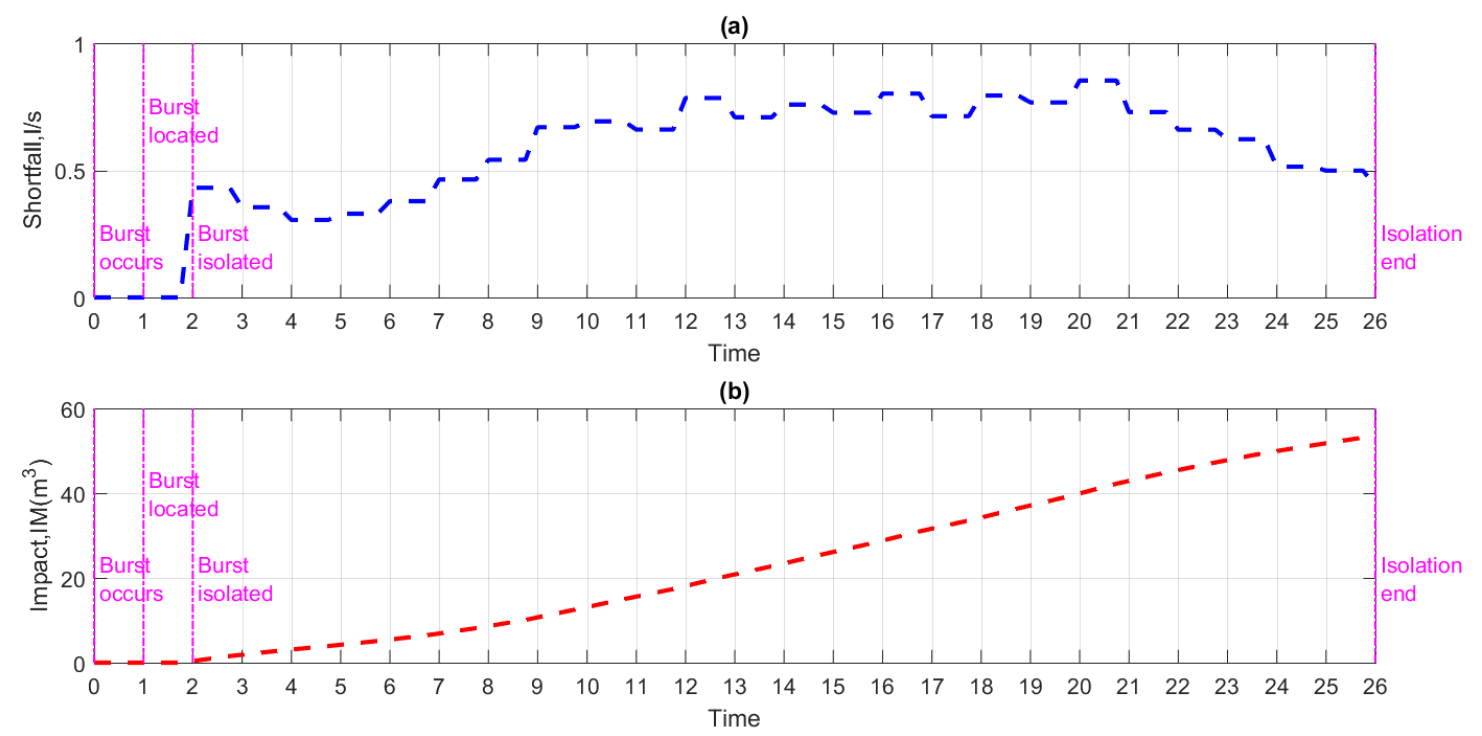

Figure 5.13. The estimated impact of isolating pipe P307 on the isolated nodes in terms of (a) shortfall (b) total cumulative volume of water undelivered.

\subsubsection{Recovery phase}

In order to mitigate the impact of pipe P307 isolation on the system (e.g., affected demand nodes located in DMA 1 and 2), the network recovery phase was initiated at 9:00 (i.e., $7 \mathrm{hr}$ after the event isolation), and lasted until the end of isolation duration.

A total of 402 intervention candidates (i.e., decision variables) are identified automatically by the offline preparation stage. This involves 247 potential overland bypasses, 152 potential isolation valves that could be manipulated and 3 PRVs (i.e., V1, V45 and V47) whose their settings could be potentially changed. Most of the above interventions are located in the two affected DMAs (1 and 2). Note that out of the total of 247 potential overland bypasses, 35 connect DMA1 with the unaffected nearby DMA5, and further 20 connect DMA2 with nearby unaffected DMA4.

All intervention candidates are assessed for further consideration in the online preparation stage. It is worth to mention that during the offline preparation stage 126 interventions available in the network were removed and that involves 60 overland bypasses, 61 isolation valves, and 3 PRVs (i.e., V1, V40 and N15). All of these candidates were located on the unaffected DMAs 3, 4, 5 and 6. 


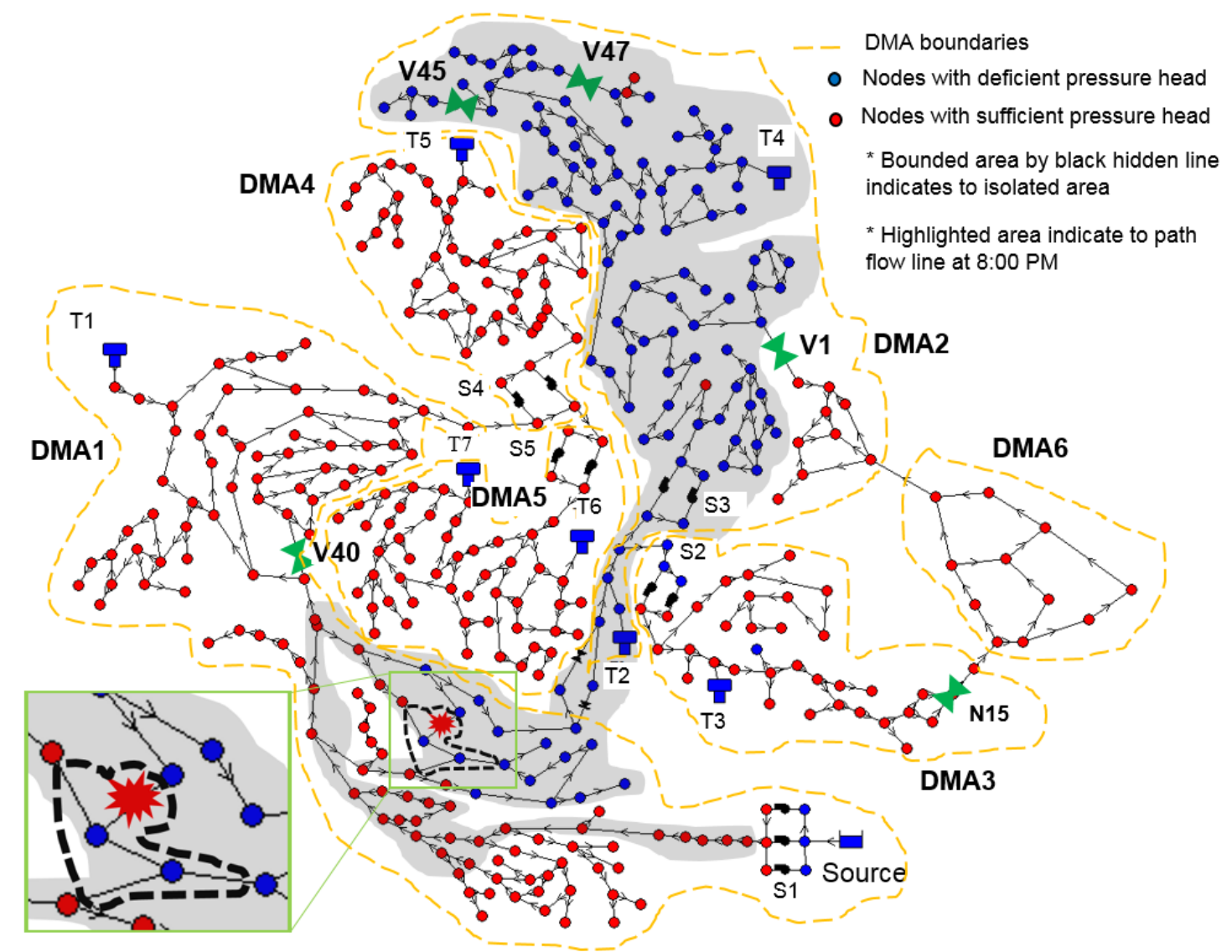

Figure 5.14. The network showing isolated area and the direction of flows for pipe P307 failure scenario at most critical time of 20:00.

The online preparation stage starts with removing intervention candidates that do not fall on the flow path (shown as shaded area in Figure 5.14) for the most critical time 20:00. This time is most critical because the demand requirement and shortfall are peaked at this time as shown in Figures 5.7 and 5.11, respectively. The above flow path was identified based on the procedure explained earlier in section 3.4.4.2. Accordingly, 128 overland bypasses, 85 isolation valves, and PRV V1 and V47 were removed from further consideration. This represents a reduction of about $53 \%$ in terms of optional intervention candidates identified in the offline preparation stage (404 in total). Most of these interventions are located at the upper part of DMA1 and trunk mains in DMAs 1 and 2, as shown in the Figure 5.14. Note until this stage of the preparation, the search space was reduced by $65 \%$ from the total interventions available in the network ( 525 in total). 
For the remaining 186 candidates (isolation valves and overland bypasses), impact $I M$ was obtained by running the EPANET hydraulic solver (based on the SIPDM) for each candidate recovery intervention individually. Finally, after ranking all interventions, the best 10 individual interventions with the lowest $I M$ values (i.e., those that produce impact less than $I_{R}$ ) were identified as shown in Figure 5.15. As it can be observed from this figure, all identified interventions reduce the volume of undelivered water to consumers (IM) in particular OV5, OV223, OV225, OV224, OV217 and OV221, when compared to the "do nothing" intervention $\left(I M_{R}=533 \mathrm{~m}^{3}\right)$. Interestingly, the best candidate intervention, which involves installing an overland bypass (OV5) between fire hydrants at $\mathrm{J} 13$ and $\mathrm{J} 11$, is very close to the isolated area. In fact, it is parallel to pipe P307 as shown in Figure 5.16. This means that identified interventions near the isolated area have more chances to reduce the negative impact, which also makes sense from an engineering point of view.

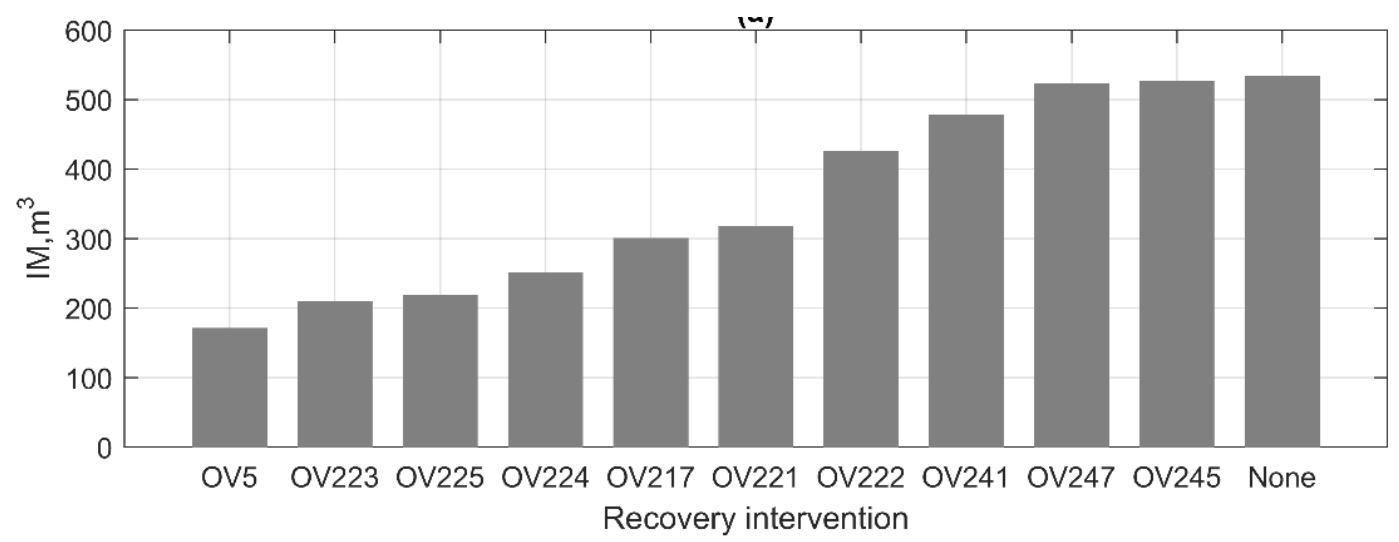

Figure 5.15. Best ranked operational recovery interventions for pipe P307 failure scenario. 


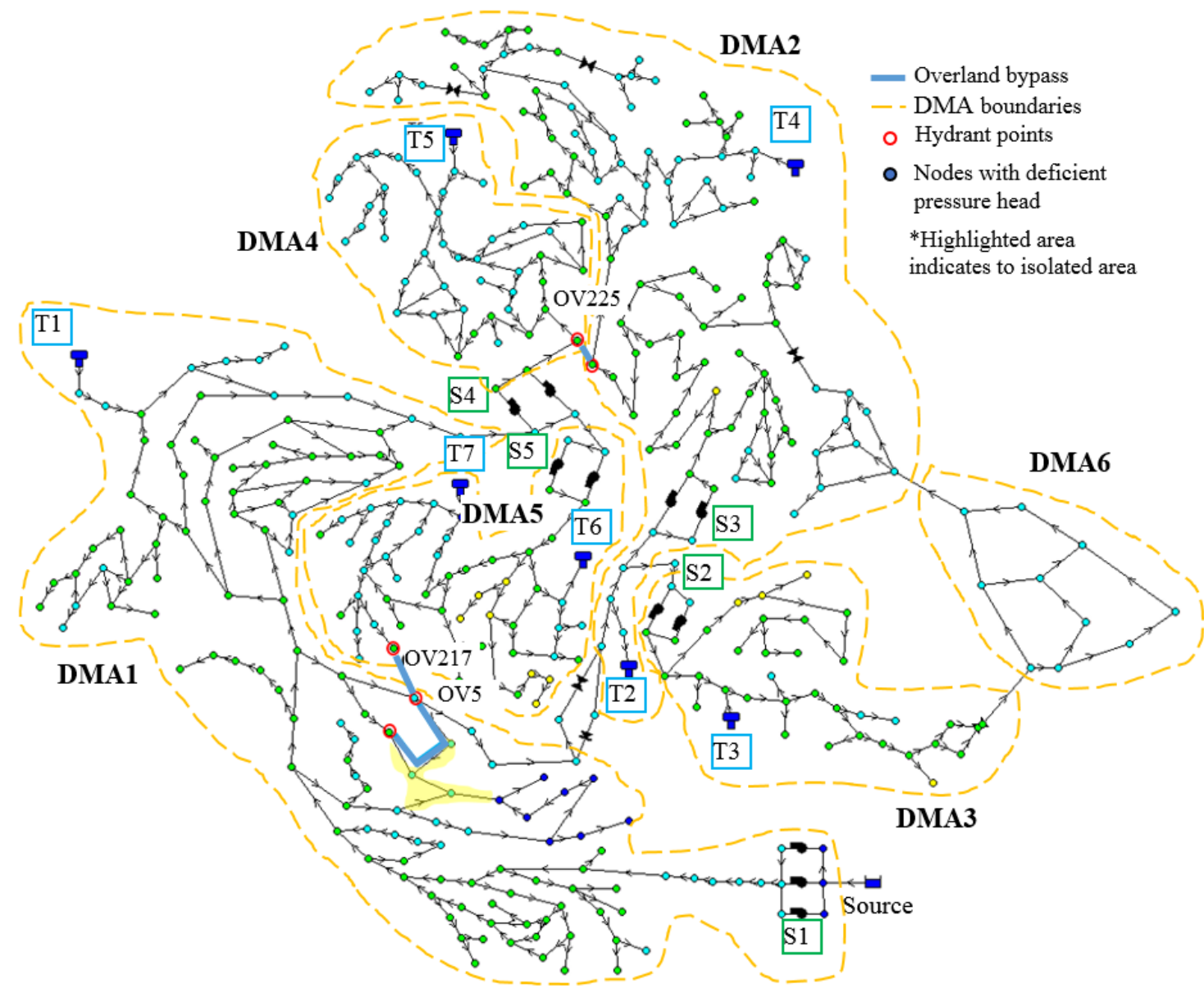

Figure 5.16. The location of recovery interventions for INV=3 (OV5, OV217, and OV225) for P307 failure scenario.

The selected 10 overland bypass intervention candidates (possible choices of 0 do not install and 1-install) are combined with the possible modifications to V45 settings (possible choices: 30.8, 32.34, 33.88, 35.42, 36.96 and $38.5 \mathrm{~m}$ ) to make the full list of possible interventions that were optimised using the NSGA-II. Given the full search space size of $2^{10 *} 6^{1}=6,144$, the population size and total number of generations were set to 20 and 150, respectively. The Pareto optimal front was obtained after 10 generations and it involves only three non-dominated solutions, as shown in Figure 5.17. The other solutions for $I N V=3,4$ and 5 are all dominated and are added to the figure for comparison purposes only.

The full model run, pre-optimisation selection involving both offline and online preparation stages in addition to optimisation itself, took approximately $30 \mathrm{~min}$ of computational time, mostly to perform relevant hydraulic solver runs. Note that this is smaller than the 1 hour time step adopted in this near real-time analysis. 
At the same time, the Enumeration method took about $8.5 \mathrm{hrs}$ to identify the equivalent Pareto front from all, 6,144 possible solutions. Note that this notably longer time prevents the use of Enumeration method in near real-time.

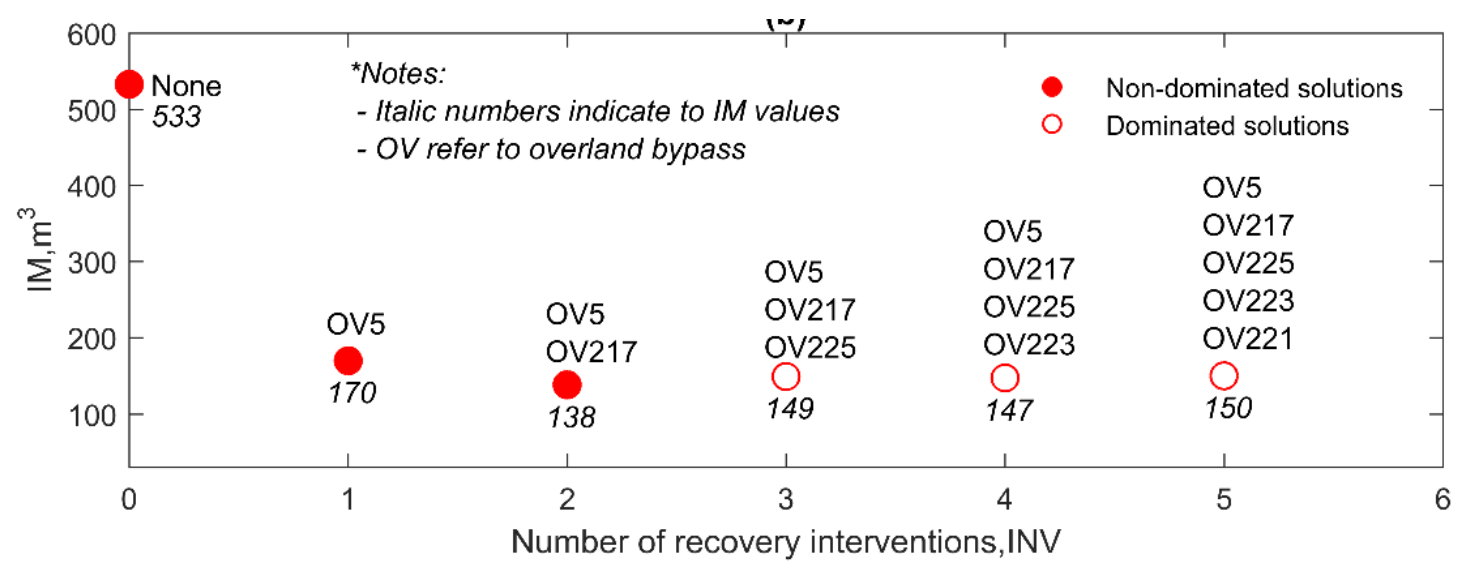

Figure 5.17. The final optimal recovery interventions for pipe P307 failure.
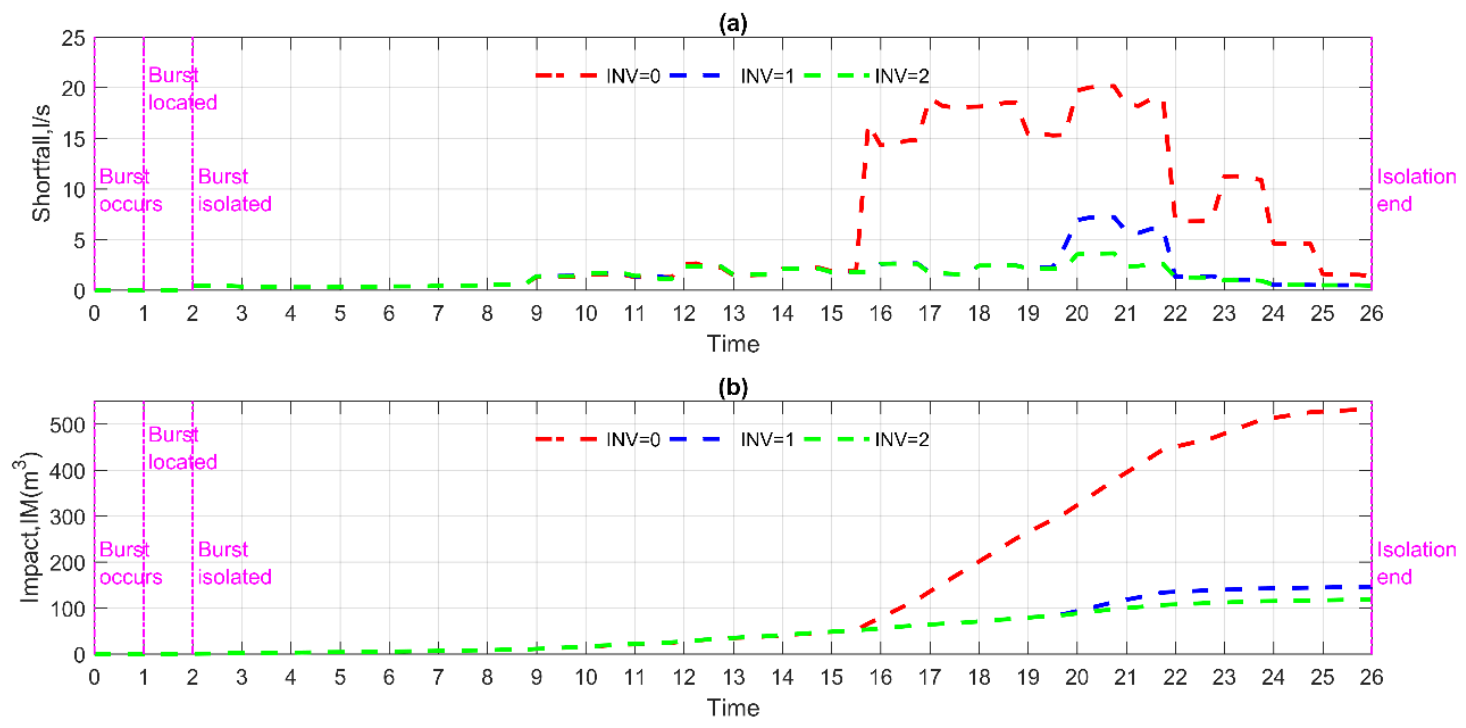

Figure 5.18. The estimated impact of pipe P307 failure on the system (including the isolated area), after introducing recovery interventions, in terms of (a) shortfall (b) total cumulative volume of water undelivered.

As it can be seen from Figure 5.17 and Figure 5.18, two specific recovery interventions (OV5 and OV217) appear in all optimal solutions. Together they reduce $I M$ from $533 \mathrm{~m}^{3}$ (for $I N V=0$ ) to a minimum value of $138 \mathrm{~m}^{3}$, as well they reduce the shortfall over the time. Furthermore, following the implementation of 
these two overland bypasses, starting from time 9:00, most nodes in DMA1 and DMA2 start to receive either the full or reduced quantity of water as shown in Figure (5.16). Note that one of these two bypasses, OV217, is connecting DMA5 with DMA1 (see Figure 5.16). This means that supply from DMA5 to DMA1 (or the combination of two DMAs) has a considerable impact on recovering deficient nodes in DMA1 to normal pressure conditions. However, this is not always true, as for example in the case of $I N V=3$ (which brings water from DMA4 to DMA2 by opening OV225), where the volume of water undelivered (IM) increased when compared to $I N V=2$. This is because the water delivered from DMA4 to DMA2 has improved situation in DMA2, but has also worsened the supply conditions in DMA4.

Figure 5.17 also shows that increasing the number of intervention combinations does not necessarily lead to a reduced volume of undelivered demand. This is clearly observed in the same table, where the dominated solutions with interventions for $I N V=3,4$ and 5, produced a larger impact reduction than the solution for $I N V=2$ did. Eventually, the solution for $I N V=2$ could be adapted as the optimal recovery option, i.e., the solution nearest to the ideal point (INV=0,IM=0), and can be proposed to the control room operator.

\subsubsection{Response in the second failure scenario}

In order to further validate the proposed response methodology and assess its computational performance, the same WDS described above was tested assuming a second failure, sudden pipe burst at pipe P424.

\subsubsection{Impact assessment}

The isolation of a burst on pipe P424 led to the unintended isolation of the pipe P53 and a non-demand node (J384) from the rest of the network. As a result, the system (in particular consumers allocated to demand nodes in DMA5) was subjected to abnormal flow conditions starting from 15:00. This can be observed from the Figure 5.19. However, the supplied volume of water to consumers remained unchanged from the original requirements during the period from 10:00 to 15:00 whilst the water depth at tanks T6 and T7 slightly fluctuated and declined as compared to normal demand stress condition in the network (see Figure 5.20). 
The two tanks were able to deliver the full demand to all nodes in the DMA5 until 14:00 (i.e., one hour after the isolation), and partial demand until 17:00, as shown in Figure 5.20. This because the modified D-Town was designed to provide full demand requirement for two hours under the power outage failure, as demonstrated by Gulodin et al (2012). Also because of the burst on pipe P424, the station $\mathrm{S} 2$ was disconnected from DMA5.

From the Figure 5.19 it can be observed that the cumulative volume of undelivered flow (IM) reached $112 \mathrm{~m}^{3}$ for 2 hours after the time of isolation (i.e., at 16:00). The cumulative volume of undelivered water increased to $226 \mathrm{~m}^{3}$ at 17:00 resulting in all 49 nodes in DMA5 suffering from undelivered demand. From this point in time onward, all consumers in DMA5 remained without water until the end of impact horizon considered (i.e., 38:00) and the total volume of undelivered water ultimately increased to approximately $3,200 \mathrm{~m}^{3}$, as shown in Figure 5.19. The reason for such a high volume of undelivered water in DMA5 is that this DMA was fully disconnected from the main water source by the pipe burst isolation. The two tanks T6 and T7 initially compensated for this failure, but ended up being completely empty at 17:00 and 15:00, respectively.
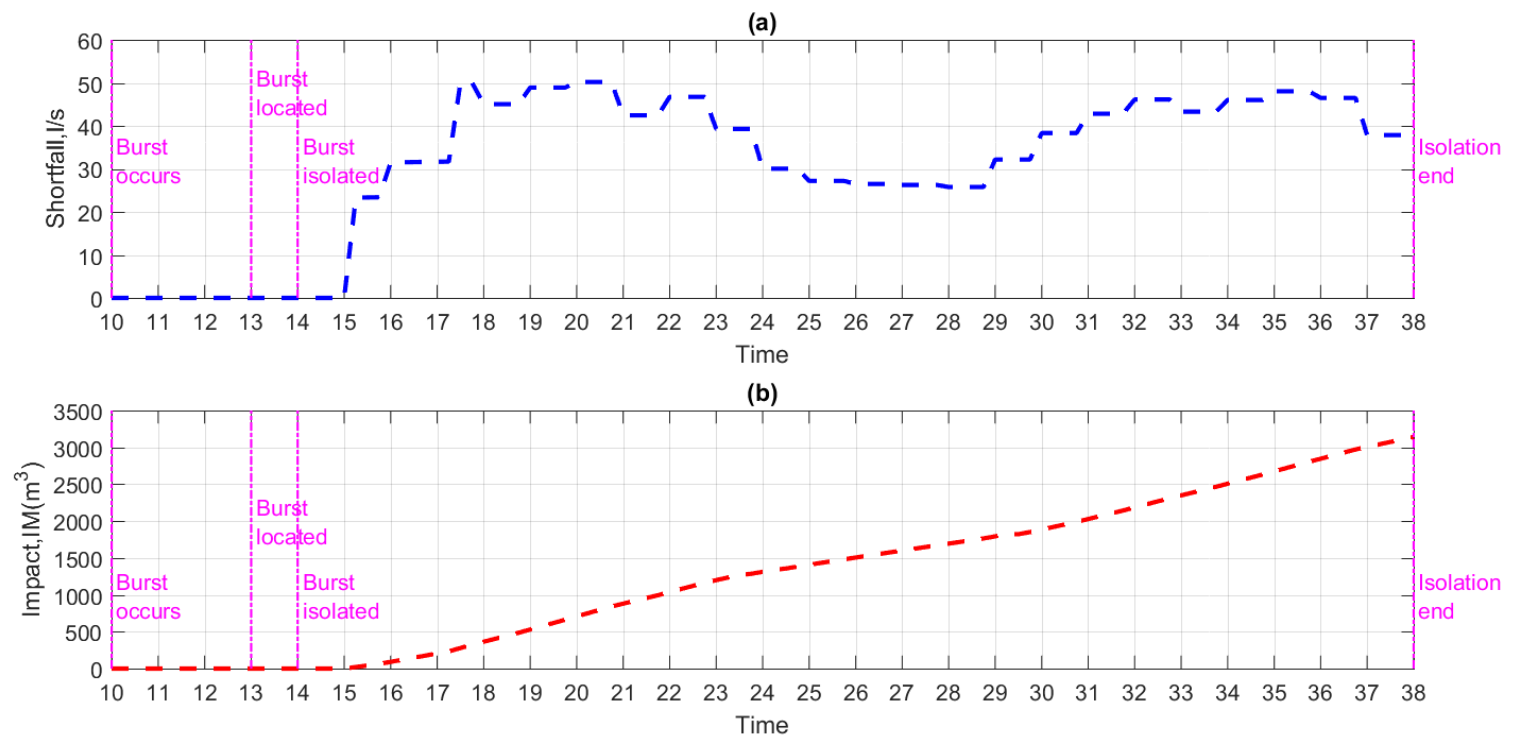

Figure 5.19. The estimated impact of isolating pipe P424 on the DMA5 nodes in terms of (a) shortfall (b) total cumulative volume of water undelivered. 


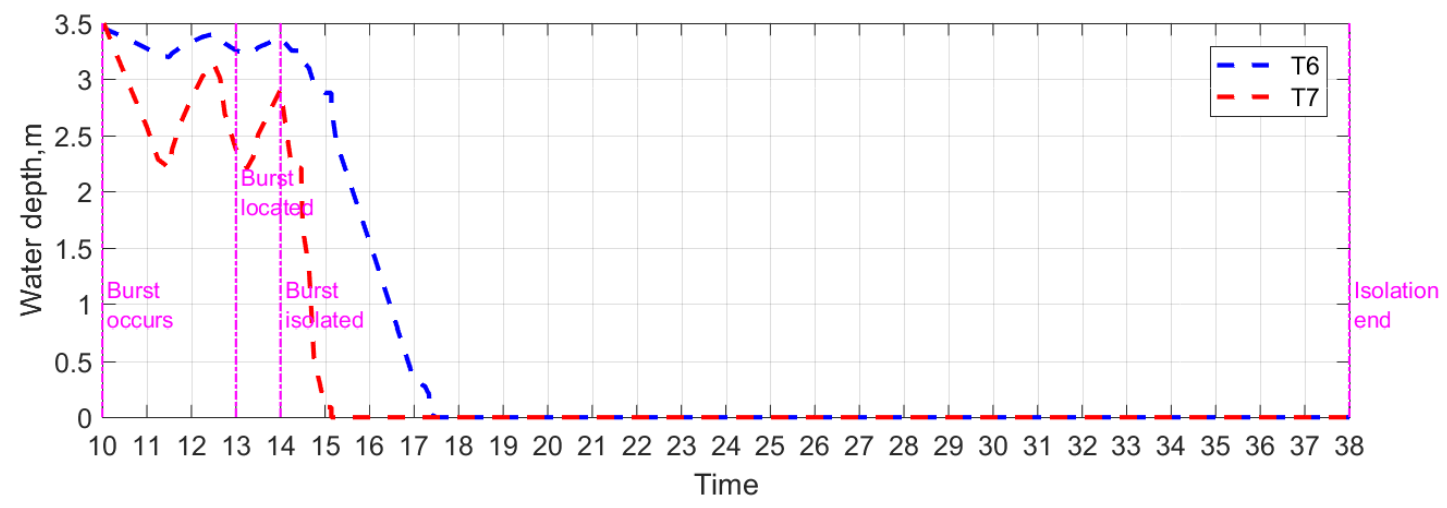

Figure 5.20. Water depth at tank T6 and T7 under pipe P424 failure scenario.

\subsubsection{Recovery phase}

In order to mitigate the impact of pipe P424 isolation, the network recovery was assumed to start at 15:00 and last until the end of isolation duration (i.e., 38:00). A total of 85 intervention candidates (i.e., decision variables) have been identified in the offline preparation stage as potential recovery solutions. Note that this represents only about $16 \%$ of the total available interventions (i.e., 525). The candidate interventions involves potentially closing 30 isolation valves, modifying one PRV (i.e., V45) setting and installing and using overland bypasses within DMA5 (30 bypasses) and between DMA5 and the neighbouring DMA1 (24 possible bypasses). The initial pressure setting of the $\mathrm{V} 45$ was fixed at $50 \mathrm{~m}$ and was increased during recovery to one of 6 following possible choices: $50,52.5$, $55,57.5,60$ and $62.5 \mathrm{~m}$. The possible decision variable values for overland bypass and isolation valve interventions are 0 for closed status and 1 for open status. Based on the flow direction in the pipes over the horizon considered (form 14:00 to 38:00), all interventions inside DMA5 were eliminated from the online preparation stage before performing individual evaluation. This is due to the fact that all components in DMA5 are disconnected from the main water source.

The evaluation of the remaining 24 candidate solutions (bypasses that connect DMAs 5 and 1) took 2 minutes of computational time, mostly to perform relevant hydraulic solver runs. The results obtained led to a conclusion that only seven of these overland bypasses reduce the impact (i.e., produce $I M<I M_{R}=3,183 \mathrm{~m}^{3}$ ). Accordingly, these seven bypasses and modified V40 settings (located in DMA1) 
are selected as eight potential optimisation candidates. The two-objective optimizer (based NSGA-II) was introduced to find the optimum Pareto combinations amongst possible interventions. The total number of hydraulic evaluations to cover the full solution search space is $2^{7 *} 6^{1}=768$. Hence, the population size and the total number of generation are set to 15 and 30, resulting in 450 evaluations. The final set of solutions was obtained only after 6 generations (i.e., 90 hydraulic evaluations) and the detail of these solutions are summarized in Figure 5.21. The figure also shows the solutions with $I N V=5,6,7$ and 8, which are all dominated by other solutions. This was done for comparison purposes.

The full model run (pre-optimisation selection and optimisation itself) took approximately $10 \mathrm{~min}$ of computational time. The Enumeration method required $64 \mathrm{~min}$ to identify the same set of optimal solutions. Note that even though the difference in computational times between optimisation and enumeration is not as large as in the first failure scenario, performing the NSG-II optimisation is still much more computationally efficient than Enumeration and is the only one feasible (given a 1 hour time step for real time analysis).

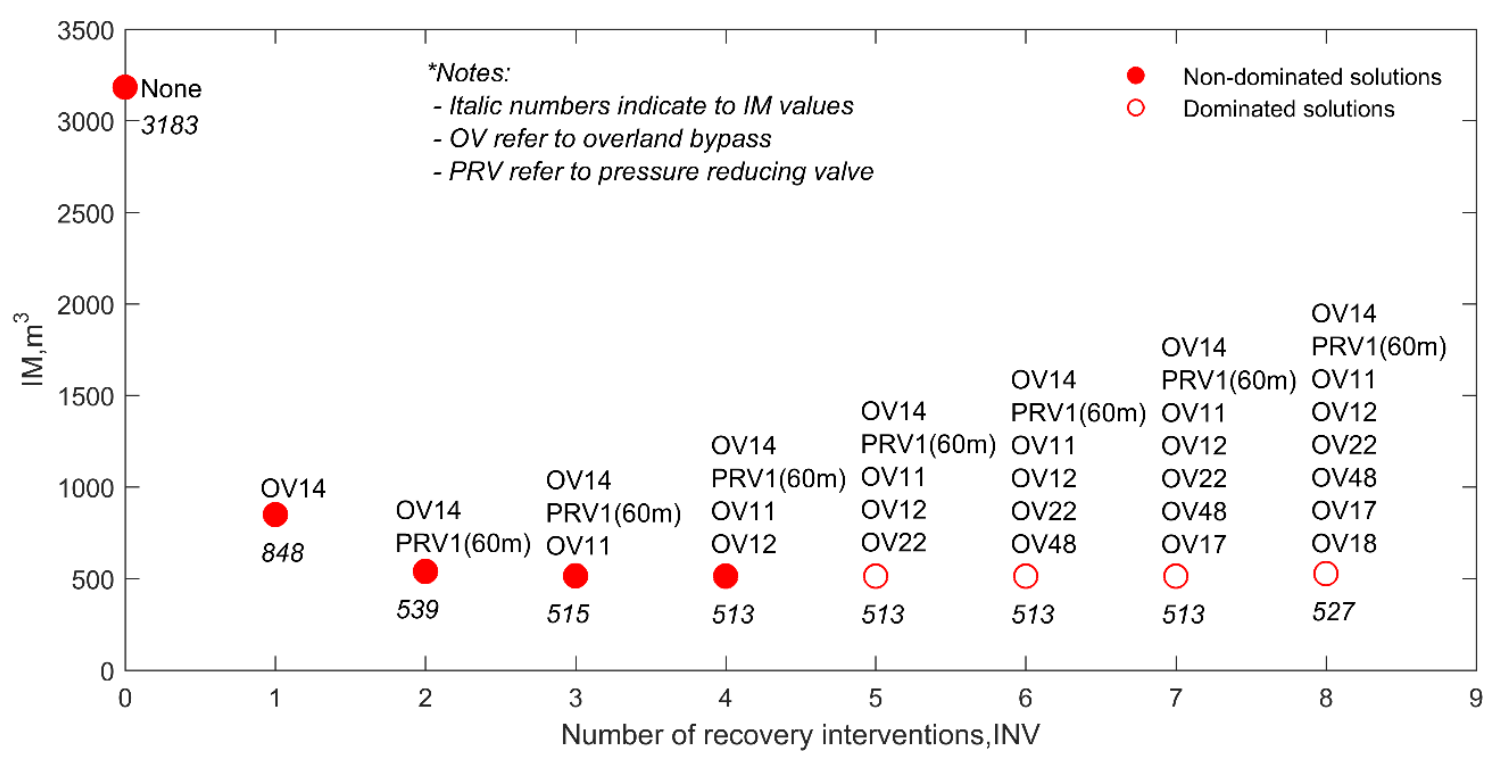

Figure 5.21. The final optimal recovery interventions for pipe P424 failure scenario. 
(a)

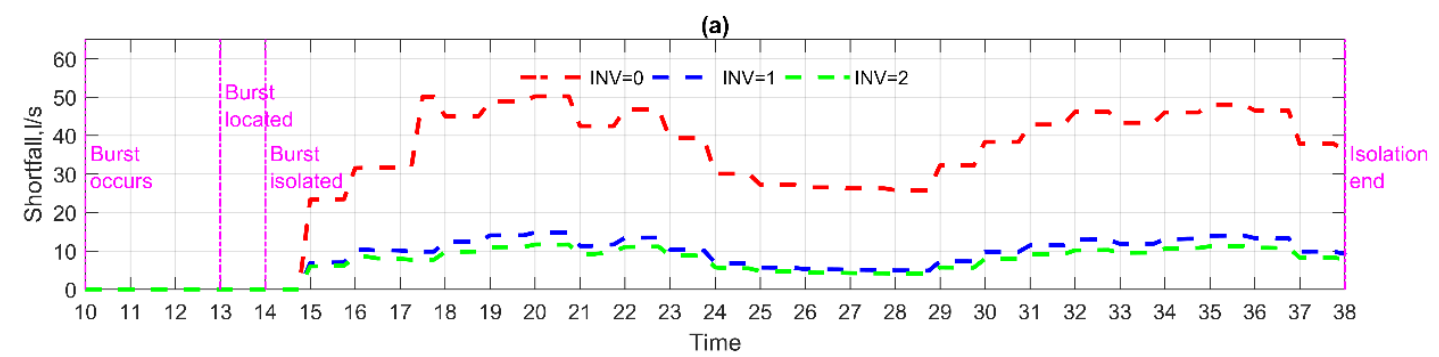

(b)

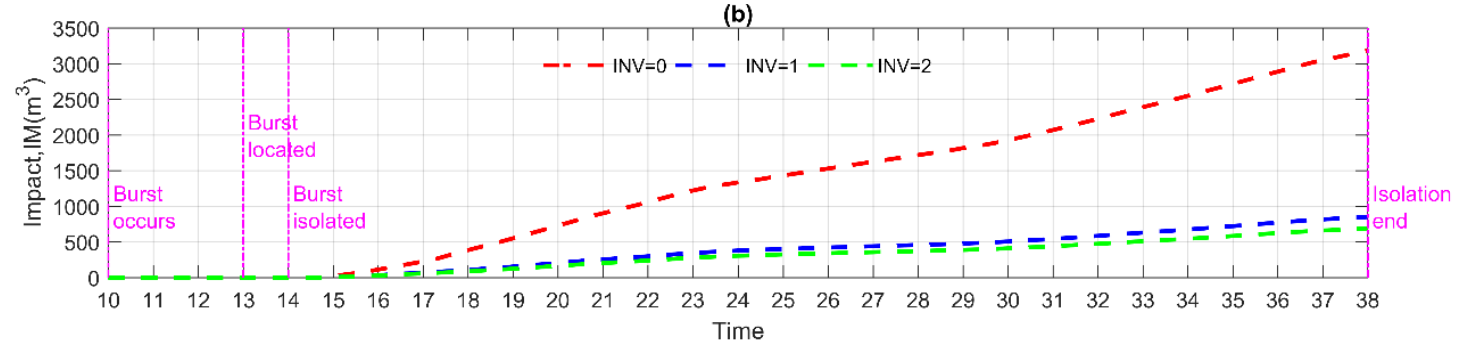

Figure 5.22. The estimated impact of pipe P424 failure on the system (including the isolated area), after introducing recovery interventions, in terms of (a) shortfall (b) total cumulative volume of water undelivered.

As shown in Figure 5.21, all eight recovery interventions $(I N V=1-8)$ reduced the impact following a pipe burst compared to the 'do nothing' option $(I N V=0)$. Interestingly, two specific interventions (overland bypass OV14 and modifying PRV1 setting to $60 \mathrm{~m}$ ) have appeared in all optimal solutions besides INV=1. This means that both interventions have a significant effect on mitigating the negative impact of failure as illustrated in Figure 5.22. Together they reduced $I M$ from $3,183 \mathrm{~m}^{3}$ (for $I N V=0$ ) to $539 \mathrm{~m}^{3}$ (for $I N V=2$ ) - see Figure 5.21. Further, from 16:00, most nodes in DMA5 started to receive either full or reduced water delivery, as shown in Figure 5.23. With $I N V=3$ (or opening OV11 as well) the

impact has further declined, with both overland bypass solutions (OV14 and OV11) connecting DMA1 with DMA5 (Figure 5.23c). This means that supply from DMA1 to DMA5 (or the combination of two DMAs) has a significant impact on recovering deficient nodes in DMA5 to normal pressure conditions as highlighted in Figure 5.23.

Having said this, increasing the number of intervention combinations does not necessarily lead to a reduced volume of undelivered demand. This is clearly observed in Figure 5.21, where solutions with interventions for $I N V=4,5,6$ and 
7 produced almost similar impact reduction as the solution for $I N V=3$ did. This is because the added overland bypasses in these interventions conveyed water from DMA1 to low hydrant points in DMA5. More interestingly increasing the number of interventions from 7 to 8 produced larger $I M$ value (as shown in Figure 5.21). This is because the additional intervention (e.g., OV18) involves bringing additional water from DMA1 to DMA5 which, in turn, negatively affects the nodes located in DMA1, which then do not receive the full required demand. Ultimately, the solution for $I N V=3$ is adapted as the optimal recovery option as the solution nearest to the ideal point $(I N V=0, I M=0)$.
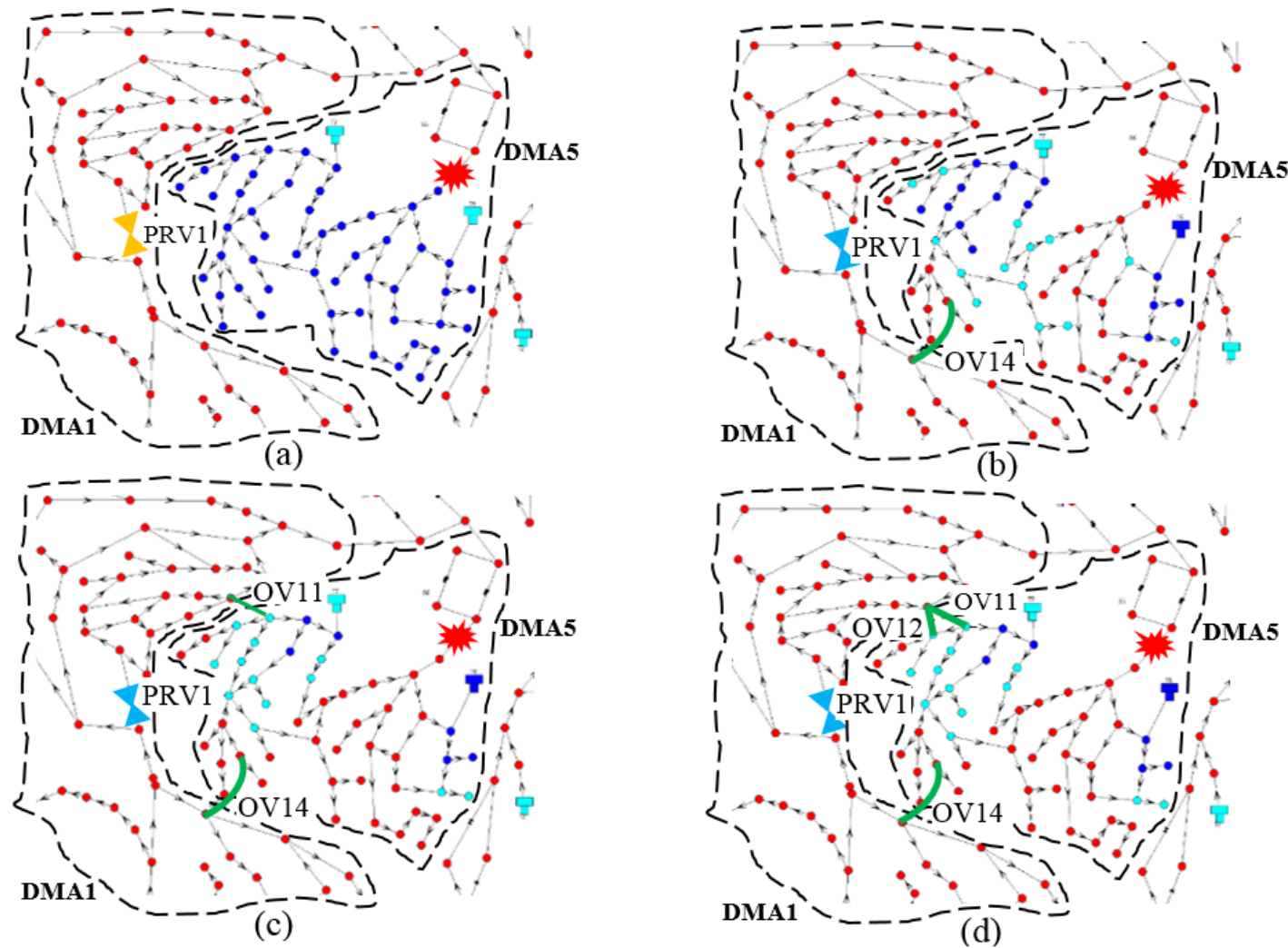

- No demand delivered

- Partial demand delivered

- Full demand delivered

- - DMA boundaries

Figure 5.23. Effect of the number of interventions on system recovery at time 15:00 for: (a) INV=0, (b) INV=2, (c) INV=3, (d) INV=4. 


\subsection{Summary}

The application of the real-time operational response methodology described in chapter 3 was tested, validated and demonstrated on a semi-real case study of D-Town network in this chapter. The network was tested under two hypothetical pipe burst failure scenarios.

The results obtained demonstrate the potential effectiveness and benefits of using the proposed response methodology. Moreover, the results shows that the proposed response methodology can effectively reduce the negative impact of unplanned failure events in water distribution networks (once these events are detected and located). This is achieved via the automated identification of optimal operational recovery interventions in near-real time.

The results obtained also show that changing the topology of a WDS by introducing recovery interventions can reduce substantially the negative impact of failures on consumers when compared to the "do-nothing" option. In addition, increasing the number of recovery interventions under contingency situations does not necessarily lead to improved network operating conditions. Finally, the results obtained demonstrate that the proposed narrowing down of response interventions based on the two preparation stages (i.e., offline and online) can effectively reduce the search space size before applying real-time optimization. The Pareto optimal interventions identified can be ultimately presented to the control room operator for making suitable decisions under contingency situations in near real-time.

In the following chapter 6 , a summary of the research work carried out and presented in this thesis is given together with the thesis main conclusions and a discussion about the directions of the future research work. 


\section{CHAPTER 6: SUMMARY, CONCLUSIONS AND FUTURE WORK RECOMMENDATIONS}

\subsection{Summary}

The aim of this thesis was to develop an efficient response methodology to reduce the impacts of unplanned failure occurrences (e.g., pipe bursts) in a WDS and restore the flow conditions in the network to normal service level once the failure has been detected and located. The response methodology proposed in this thesis is based on the current practice in the UK water industry and can assist WDS operators in making more informed decisions and prioritise interventions/ actions more effectively.

Two stages were defined for the operational response: (i) the isolation of a potential failure event with associated segment (if any), followed by (ii) the recovery stage. The isolation includes finding the nearest set of valves to isolate the failure location and identify the interrupted/isolated consumers from water sources because of the failure event. The recovery stage involves selecting the optimal combination of individual operational interventions, with the aim to minimize both the negative impact of reduced/interrupted supply over a predefined impact horizon (estimated as the volume of water undelivered) and the associated operational costs (estimated in this thesis via the weighted total number of operational interventions required). This two-objective optimisation problem was solved by using the NSGA-II.

The optional choices considered are opening valves within the affected DMA and between the affected and nearby DMAs, resetting pressure at the outlet of PRV(s) and installing temporary overland bypasses between suitable hydrant points. The optimal set of interventions was determined using a new methodology presented in this thesis that makes use of multi-objective optimisation coupled with a pressure-driven model (PDM) hydraulic solver to evaluate potential solutions.

The developed Single Iteration PDM (SIPDM) approach for assessing the negative impacts associated with the failure events is based on using a specific set of elements added to each deficient pressure demand node in the network. This enables modelling the minimum pressure head below which no flow occurs, 
the required pressure above which the full demand required is delivered, and the partial flow conditions for the pressure heads in between these two characteristic values. The SIPDM approach suggested here uses the Wagner et al. (1988) relationship for the latter, although other relationships could be modelled if deemed more suitable.

The overall response methodology is presented chapter 3 and was tested, validated and demonstrated on a case study of D-Town network in chapter 5 . The SIPDM approach presented, validated and demonstrated in chapter 4.

\subsection{Conclusions}

The work carried out in this thesis forms a useful contribution to the water system research field through the key conclusions shown in the following two subsections.

\subsubsection{Real-time operational response methodology}

The operational real-time response methodology proposed in this thesis is intended to provide support to operators when making relevant decisions, i.e. help them do their job even better under emergency condition. This applies especially to cases when failures are more complex in nature (e.g., affecting a larger part of the water distribution system and customers) and decisions need to be made quickly. The following main conclusions can be made from the results obtained in chapter 5 :

1. The proposed operational response methodology can effectively reduce the negative impact of unplanned failure events in water distribution networks (once these events are detected and located). This is achieved by automated identification of optimal operational recovery interventions in near-real time, i.e. identify and shortlist suitable candidate operational interventions prior to optimisation in near real-time short time. The Pareto optimal interventions are identified by addressing the trade-off between the reduced negative impact due to interventions applied and the associated intervention costs. These could be ultimately presented to the control room operator for making suitable decisions under contingency 
situations in near real-time. This, in turn, is expected to result in improved operational efficiency and emergency preparedness, reduced water loss and shortened response time.

2. The Pareto optimal recovery interventions considered here (valve manipulation, PRV resetting and installation of temporary overland bypasses between fire hydrants) can, in some cases, result in changing temporarily the topology of the analysed WDS. It has been shown that this can be very beneficial, i.e. it can reduce substantially the negative impact of failures on consumers (when compared to the "do-nothing" option). At the same time, increasing the number of recovery interventions under contingency situations does not necessarily lead to improved network operating conditions. The reason for this is that most of impact reduction seem to be achieved by a small number of carefully selected interventions.

3. The new intervention selection methodology shown in section 3.4.4 can effectively and considerably reduce the number of the interventions (i.e. decision variables) in near real-time for a given network and consequently reduce the search space during the process of finding optimal intervention solutions under emergency failure conditions. This is beneficial as the time available to perform optimisation in near-real-time is limited. In particular helpful in the case of more complex networks and events, affecting a larger area/number of consumers and/or when the response needs to be identified quickly.

4. The developed segmentation method shown in section 3.3 is able to: (1) delineate segments (including both regular and unintentional) containing failed components (e.g., pipe burst) for a water distribution system; (2) identify the location of all necessary valves to isolate the failure event; (3) provide numerical performance measures for assessing failure impacts in terms of the number of customers out of service (i.e., isolated) and (4) utilize the industry standard EPANET software to perform hydraulic calculations to combine both topological and hydraulic failures. The segmentation method can handle very large size networks due to simple and scalable matrix calculations and search evaluations used. 


\subsubsection{Pressure driven analysis}

A simple and robust PDM methodology and tool (SIPDM) is developed here to model the behaviour of real networks under pressure deficient conditions resulting from the failure events in a WDS (e.g., pipe bursts). The results obtained in chapter 4 lead to the following main conclusions:

1. The proposed SIPDM approach is able to simulate effectively and efficiently the WDN under both normal and pressure-deficient conditions and in both steady-state and extended period simulations. The predicted flows and pressures in the network are accurate, numerically stable, and obtained in a computationally efficient manner.

2. When compared to other PDM methods reported in the literature, the SIPDM possesses the following key advantages: (1) it predicts the same or more-accurate pressures and flows under pressure-deficient conditions; (2) it does this in a generally more computationally efficient manner, especially when applied to larger pipe networks; and (3) unlike some PDM methods, it can be used for extended period simulation, i.e., not just steady-state hydraulic analysis.

3. When SIPDM is compared to a DDM type approach, the following key differences are observed: (1) SIPDM predicts the same pressures and flows under pressure-sufficient conditions, and more realistic and accurate pressures and flows under pressure-deficient conditions, for both steadystate and EPS analysis; (2) As expected, SIPDM is slower than DDM by about $30 \%$ which is acceptable when compared to other PDM methods tested.

4. The proposed SIPDM approach works well with the widely used EPANET2.0 software, although there is no reason why it should not work well with other popular hydraulic solvers, including the commercial ones. The reason for this is the generic nature and relative simplicity of the SIPDM approach and the fact that it does not require any hydraulic solver source code modifications. 
Based on the aforementioned, the SIPDM approach proposed lends itself naturally to tackling practical problems such as WDN reliability, optimal location of isolation valves, and multi-objective optimization.

\subsection{Future Work Recommendations}

The recommendations for further work regarding the proposed real-time operational response methodology are as follows:

- Further evaluation and demonstration of the benefits of response methodology, especially on more complex real-life case studies and under actual online failure environment conditions. This will provide opportunities to address issues such as inoperable valves and considering other available recovery intervention options (such as booster pumps and using emergency volume in storage tanks).

- Improved impact modelling of operational interventions which in this thesis has been limited to quantifying the volume of water not delivered only. Additional impacts need to be considered (e.g., traffic interruptions due to a large burst, damage to local properties or shops flooding caused by a pipe burst, etc.).

- The cost model for operational interventions should be modified to make these more realistic. This can be done by involving elements such as the travel time of the network operators from the control room to the location where the recovery intervention is needed, the time required to install an overland bypass or modify the setting for a particular isolation valve or PRV, to name the few.

- Wider range of possible operational interventions need to be considered (e.g., provision of bottled water or water delivered by cisterns, etc.), together with associated impact and cost models.

- Additional and more realistic criteria (e.g., water quality considerations, sensitivity of different consumers, behaviour of consumers affecting the 
water demand under failure conditions, resilience, to name the few) could be used to drive the selection of operational recovery interventions.

Regarding the pressure-driven model, further research should focus on the relationship between pressure and demand. This relationship has physical basis, but it is also has an element of human behaviour involved. Demands are an estimate based on metred usage. As pressure drops, there is a physical element involved in the ability to deliver the water to a location. However, as pressure drops, some of the demand may completely to be stop or be reduced dramatically. For example, if a person perceives lower pressures some normal practices may be reduced. These behaviour should also be considered when determining the proper relationship between demand and pressure in lowpressure conditions. Water utilities generally keep records on pipe failures or other service disruptions. These data, in conjunction with other system data such as tank levels or observations of water meters, could be used more effectively to estimate the above relationship. 


\section{References}

Abdy Sayyed, M. A. H., and Gupta, R. (2013). "Predicting deficientcondition performance of water distribution networks." Civil Eng. Infrastruct. J., 46(2), 626-631.

Ackley, J. R. L., Tanyimboh, T. T., Taher, B., and Templeman, A. B. (2001). "Head-driven analysis of water distribution systems." Proc., Computer and Control in Water Industry, Vol. 1, Research Studies Press, Leicester, U.K., 183-192.

Alfonso, L., A. Jonoski, and D. Solomatine (2010). "Multiobjective Optimization of Operational Responses for Contaminant Flushing in Water Distribution Networks" J. Water Resour. Plan. Manag., 136(1), 48-58, doi:10.1061/(ASCE)0733-9496(2010)136:1(48).

Alonso, J. M., et al. (2000). "Parallel computing in water network analysis and leakage minimization.” J. Water Resour. Plann. Manage., 126(4), 251-260.

Alvisi, S., Creaco, E., and Franchini, M. (2011). "Segment identification in water distribution systems." Urban Water J., 8(4), 203-217.

Alvisi, S., M. Franchini, M. Gavanelli, and M. Nonato (2012). "Near-optimal scheduling of device activation in water distribution systems to reduce the impact of a contamination event" J. Hydroinformatics, 14(2), 345, doi:10.2166/hydro.2011.147.

Amarasinghe, P., Liu, A.; Egodawatta, P., Barnes, P., McGree, J., Goonetilleke. "A Quantitative assessment of resilience of a water supply system under rainfall reduction due to climate change "J. Hydrol. 2016, 540, 1043-1052.

American National Standards Institute, (2010). "Risk Analysis and Management for Critical Asset Protection (RAMCAP) Standard for Risk and Resilience Management of Water and Wastewater Systems" American Water Works Association, Denver, CO.

American Water Works Association (2001). "Emergency planning for water utilities" AWWA Manual M19, fourth edition, Denver, USA. 
Ang, W. K., and Jowitt, P. W. (2006). "Solution for water distribution systems under pressure-deficient conditions." J. Water Resour. Plann. Manage., 10.1061/(ASCE)0733-9496(2006)132:3(175), 175-182.

Asefa, T., Clayton, J., Adams, A., and Anderson, D. (2014). "Performance evaluation of a water resources system under varying climatic conditions: Reliability, resilience, vulnerability and beyond." J. Hydrol., 508, 53-65.

Awumah, K., Goulter, I. (1989). "Redundancy and reliability of water distribution networks: An entropy based approach" In: Ports, M. (ed.) Proceedings of 1989 ASCE National Conference on Hydraulic Engineering, pp. 768-773, New Orleans, August 14-18

Bakker, M., J. H. G. Vreeburg, K. M. van Schagen, and L. C. Rietveld (2013). "A fully adaptive forecasting model for short-term drinking water demand" Environ. Model. Softw., 48, 141-151, doi:10.1016/j.envsoft.2013.06.012.

Bao, Y., and Mays, L. W. (1990). "Model for water distribution system reliability." J. Hydraul. Eng., 10.1061/(ASCE)0733-9429(1990)116: 9(1119), 1119_ 1137.

Baranowski, T. M. and E. J. LeBoeuf. (2008). "Consequence management utilizing optimization" Journal of Water Resources Planning and Management, 134(4), 389-394.

Berglund, E. (2015). "Using agent-based modeling for water resources planning and management." J. Water Resour. Plann. Manage., 10 .1061/(ASCE)WR.1943-5452.0000544, 4015025.

Bhave, P. R. (1981). "Node flow analysis of water distribution systems." J. Transp. Eng., 107(4), 457-467.

Bicik, J., D. A. Savić, and Z. Kapelan (2010). "Operation of Water Distribution Systems Using risk-based Decision Making" J. Chem. Inf. Model., 53(9), 1689-1699.

Bicik, J., Z. Kapelan, and D. A. Savić (2009). "Operational Perspective of the Impact of Failures in Water Distribution Systems" in World Environmental and Water Resources Congress 2009, pp. 1-10, American Society of Civil Engineers, Reston, VA. 
Blackmore, J.M., Plant, R.A. (2008). "Risk and resilience to enhance sustainability with application to urban water systems." J. Water Resour. Plan. Manag., 134, 224-233.

Bouchart, F., and I. Goulter (1991). "Reliability Improvements in Design of Water Distribution Networks Recognizing Valve Location" Water Resour. Res., 27(12), 3029-3040, doi:10.1029/91WR00590.

Boulos, P. F., and A. N. Wiley (2013). "Can We Make Water Systems Smarter?" Opflow, 39(3), 20-22, doi:10.5991/OPF.2013.39.0015.

Boulos, P.F.; Jacobsen, L.B.; Heath, J.E.; Kamojjala, S. (2014). "Real-time modeling of water distribution systems: A case study" J. Am. Water Works Assoc. 106, 119-120.

Brualdi, R.A. and Ryser, H.J.. (1991). "Combinatorial matrix theory" Cambridge University Press.

Bruneau, M., Chang, S.E., Eguchi, R.T., Lee, G.C., O’Rourke, T.D., Reinhorn, A.M., Shinozuka, M., Tierney, K., Wallace, W.A., von Winterfeldt, D.,. (2003). "A framework to quantitatively assess and enhance the seismic resilience of communities" Earthq. Spectra, 19, 733-752.

Butler, D., S. Ward, C. Sweetapple, M. Astaraie-Imani, K. Diao, R. Farmani, and G. Fu (2017). "Reliable, resilient and sustainable water management: the Safe \& SuRe approach" Glob. Challenges, 1(1), 63-77, doi:10.1002/gch2.1010.

Cabinet Office (2011) "Keeping the county running: natural hazards and infrastructure" A guide to improving the resilience of critical infrastructure and essential services. Cabinet Office.

Casal, A. (2016). "Integrated Management of Urban Wastewater Systems: Exploring Reliable, Resilient and Sustainable Strategies for an Uncertain Future" PhD Thesis, University of Exeter, UK.

Cassa, A. M., van Zyl, J. E., and Laubscher, R. F. (2010). "A numerical investigation into the effect of pressure on holes and cracks in water supply pipes." Urban Water Journal, 7(2), 109-120. 
Centre for Water Systems (CWS) (2018). "Benchmarks", http://emps.exeter.ac.uk/engineering/research/cws/downloads/benchmarks/ , University of Exeter, (accessed 17 March 2018).

Chandapillai, J. (1991). "Realistic simulation of water distribution system." J. Transp. Eng., 10.1061/(ASCE)0733-947X(1991)117:2(258), 258-263.

Cheng, W.-C., N.-S. Hsu, W.-M. Cheng, and W. W.-G. Yeh (2009). "A flow path model for regional water distribution optimization" Water Resour. Res., 45(9), 1-12, doi:10.1029/2009WR007826.

Cheung, P., Van Zyl, J. E., and Reis, L. F. R. (2005). "Extension of EPANET for pressure driven demand modeling in water distribution system." Proc., 8th Int. Conf. on Computing and Control in the Water Industry CCWI05 Water Management for the 21st Century, Vol. 1, Centre for Water Systems, Univ. of Exeter, Exeter, U.K.

Ciaponi, C.; Creaco, E. "Comparison of Pressure-Driven Formulations for WDN Simulation." Water 2018, 10, 523.

Cimellaro, G. P., Tinebra, A., Renschler, C., and Fragiadakis, M. (2015). "Resilience-based design of an urban water distribution system." J. Struct. Eng., 10.1061/(ASCE)ST.1943-541X.0001433, C4015014.

Collins, M., Cooper, L., Helgason, R., Kennington, R., and Leblanc, L. (1978). "Solving the pipe network analysis problem using optimization techniques." Manage. Sci., 24(7), 747-760.

Cook, D. M., P. S. Husband, and J. B. Boxall (2016). "Operational management of trunk main discolouration risk", Urban Water J., 13(4), 382-395, doi:10.1080/1573062X.2014.993994.

Creaco, E., Franchini, M., and Alvisi, S. (2010). "Optimal placement of isolation valves in water distribution systems based on valve cost and weighted average demand shortfall." Water Resour. Manage., 24(15), 4317-4338.

Creaco, E., Franchini, M., and Alvisi, S. (2012). "Evaluating water demand shortfalls in segment analysis." Water Resour. Manage, 26(8), 2301-2321.

Critical Infrastructure Partnership Advisory Council. (2009). "All-Hazard Consequence Management Planning for the Water Sector: Preparedness, 
Emergency Response, and Recovery" CIPAC Workshop. US Department of Homeland Security, Washington DC.

Deb, K., A. Pratap, S. Agarwal, and T. Meyarivan (2002). "A fast and elitist multiobjective genetic algorithm: NSGA-II" IEEE Trans. Evol. Comput., 6(2), 182-197, doi:10.1109/4235.996017.

Diao, K., C. Sweetapple, R. Farmani, G. Fu, S. Ward, and D. Butler (2016). " Global resilience analysis of water distribution systems" Water Res., 106, 383-393, doi:10.1016/j.watres.2016.10.011.

Drinking Water Inspectorate (2011). "Provision of alternative supplies in emergency and non emergency situations" London, UK.

Duval, A. M. (1988). "A directed graph version of strongly regular graphs" J. Comb. Theory, Ser. A, 47(1), 71-100, doi:10.1016/0097-3165(88)90043-X.

Elhay, S., Piller, O., Deuerlein, J., and Simpson, A. (2015). "A robust, rapidly convergent method that solves the water distribution equations for pressuredependent models." J. Water Resour. Plann. Manage., 10.1061/(ASCE)WR.1943-5452.0000578, 04015047.

Environment. Department of Mathematics and Statistics, University of Maryland Baltimore County, Technical Report TR96-01.

Even, S. (2011). "Graph algorithms" ,2nd Ed., Cambridge University Press, New York.

Farmani R, Walters GA, Savic DA. (2006). "Evolutionary multi-objective optimization of the design and operation of water distribution network: total cost vs. reliability vs. water quality" Journal of Hydroinformatics, volume 8 , pages 165-179.

Farmani, R., Walters, G. A., and Savić, D. A. (2005). "Trade-off between Total Cost and Reliability for Anytown Water Distribution Network." Journal of Water Resources Planning and Management, 131(3), 161-171.

Fu, G., Kapelan, Z., and Reed, P. (2012). "Reducing the complexity of multiobjective water distribution system optimization through global sensitivity analysis." J. Water Resour. Plann. Manage., 138(3), 196-207. 
Fu, G., Z. Kapelan, J. R. Kasprzyk, and P. M. Reed (2013). "Optimal design of water distribution systems using many-objective visual analytics" J. Water Resour. Plann. Manag., 139(December), 624-633, doi:10.1061/(ASCE)WR.1943-5452.0000311.

Fujiwara, O., and Ganesharajah, T. (1993). "Reliability assessment of water supply systems with storage and distribution networks." J. Water Resour. Res., 29(8), 2917-2924.

Fujiwara, O., and J. Li (1998). "Reliability analysis of water distribution networks in consideration of equity, redistribution, and pressure-dependent demand" Water Resour. Res., 34(7), 1843-1850, doi:10.1029/98WwR00908.

Gallo, G., and S. Pallottino (1988). 2Shortest path algorithms" Ann. Oper. Res., 13(1), 1-79, doi:10.1007/BF02288320.

Gao, T. (2013). "Efficient identification of segments in water distribution networks." J. Water Resour. Plann. Manage., 10.1061/(ASCE)WR .19435452.0000395, 04014003.

Geem, Z. W. (2009). "Particle-swarm harmony search for water network design." Eng. Optimiz., 41(4), 297-311.

Germanopoulos, G. (1985). "A technical note on the inclusion of pressure dependent demand and leakage terms in water supply network models." $J$. Civil Eng. Syst., 2(3), 171-179.

Gheisi, A., Forsyth, M., and Naser, G. (2016). "Water distribution systems reliability: A review of research literature." J. Water Resour. Plann. Manage., 10.1061/(ASCE)WR.1943-5452.0000690, 04016047.

Giacomello, C., Kapelan, Z., and Nicolini, M. (2013). "Fast hybrid optimisation method for effective pump scheduling." J. Water Resour. Plann. Manage., 10.1061/(ASCE)WR.1943-5452.0000239, 175-183.

Gibbs, M. S., H. R. Maier, and G. C. Dandy (2015). "Using characteristics of the optimisation problem to determine the Genetic Algorithm population size when the number of evaluations is limited" Environ. Model. Softw., 69, 226239, doi:10.1016/j.envsoft.2014.08.023. 
Giustolisi, O., Kapelan, Z., and Savic, D. (2008). "Algorithm for automatic detection of topological changes in water distribution networks." J. Hydrol. Eng., 10.1061/(ASCE)0733-9429(2008)134:4(435), 435-446.

Giustolisi, O., Savic, D., and Kapelan, Z. (2008). "Pressure-driven demand and leakage simulation for water distribution networks." J. Hydraul. Eng., 10.1061/(ASCE)0733-9429(2008)134:5(626), 626-635.

Giustolisi, O., and D. Savic (2010). "Identification of segments and optimal isolation valve system design in water distribution networks" Urban Water J., 7(1), 1-15, doi:10.1080/15730620903287530.

Giustolisi, O., Savic, D. A., Berardi, L., and Laucelli, D. (2011). "An Excelbased solution to bring water distribution network analysis closer to users." Proc., Computer and Control in Water Industry, Exeter Press, Exeter, U.K.

Gorev, N. B., and Kodzhespirova, I. F. (2013). "Noniterative implementation of pressure-dependent demands using the hydraulic analysis engine of EPANET 2." J. Water Resour. Manage., 27(10), 3623-3630.

Grothmann, T., Reusswig, F., (2006). "People at risk of flooding: why some residents take precautionary action while others do not" Nat. Hazards, 38(12), 101-120.

Guidolin, M., Fu, G., and Reed, P. (2012). "Parallel evolutionary multiobjective optimization of water distribution system design." Water Distribution Systems Analysis 2012, Adelaide, Australia.

Gunawan, I., Schultmann, F., and Zarghami, S. A. (2017). "The four Rs performance indicators of water distribution networks." International Journal of Quality \& Reliability Management, 34(5), 720-732.

Gupta, R., and Bhave, P. R. (1996). "Comparison of methods for predicting deficient-network performance." J. Water Resour. Plann. Manage., 10.1061/(ASCE)0733-9496(1996)122:3(214), 214-217.

Hayuti, M., Naga, D., Zhang, Y., and Burrows, R. (2008). "An evaluation of the robustness of the sample U.K. water distribution system configurations to operational stresses." Water Distribution Systems Analysis Symp. 2006, Cincinnati. 
Hutton, C. J., and Z. Kapelan (2015). "A probabilistic methodology for quantifying, diagnosing and reducing model structural and predictive errors in short term water demand forecasting" Environ. Model. Softw., 66, 87-97, doi:10.1016/j.envsoft.2014.12.021.

IPCC. (2012). "Managing the Risks of Extreme Events and Disasters to Advance Climate Change Adaptation. Special Report of the Inter-governmental Panel on Climate Change" Cambridge University Press, New York.

Isaacs, L. T., and Mills, K. G. (1980). "Linear theory methods for pipe network analysis." J. Hydraul. Div., 106(7), 1191-1201.

Islam, M. S., Sadiq, R., Rodriguez, M. J., Najjaran, H., and Hoorfar, M. (2013). "Reliability assessment for water supply systems under uncer- tainties." $J$. Water Resour. Plann. Manage., 10.1061/(ASCE)WR.1943 -5452.0000349, 468-479.

J. Kaldenbach, L.E. Ormsbee, "Automated segmentation analysis of water distribution systems", World Environ. and Water Resour. Congress, 2012 629- 634.

Jayaram, N., and K. Srinivasan (2008). "Performance-based optimal design and rehabilitation of water distribution networks using life cycle costing" Water Resour. Res., 44(1), doi:10.1029/2006WR005316.

Jeong, H. S., and D. M. Abraham (2009). "Water Rationing Model for Consequence Minimization of Water Infrastructure Destruction" J. Water Resour. Plan. Manag., 135(2), 80-89, doi:10.1061/(ASCE)07339496(2009)135:2(80).

Jeong, H. S., J. Qiao, D. M. Abraham, M. Lawley, J.-P. Richard, and Y. Yih (2006). "Minimizing the Consequences of Intentional Attack on Water Infrastructure" Comput. Civ. Infrastruct. Eng., 21(2), 79-92, doi:10.1111/j.1467-8667.2005.00419.

Jinesh Babu, K. S., and Mohan, S. (2012). "Extended period simulation for pressure-deficient water distribution network." J. Comput. Civil Eng., 10.1061/(ASCE)CP.1943-5487.0000160, 498-505. 
Johansson (2010). "Risk and vulnerability analysis of interdependent technical infrastructures. Addressing socio-technical systems" Doctoral thesis, Lund University.

Jones, R.N., Preston, B.L., (2011). "Adaptation and risk management" Wiley Interdisciplinary Reviews - Climate Change 2(2), 296-308.

Jun, H. (2005). "Strategic valve locations in a water distribution system".

Jun, H., and Loganathan, G. V. (2007). "Valve-controlled segments in water distribution systems." J. Water Resour. Plann. Manage., 10.1061 /(ASCE)0733-9496(2007)133:2(145), 145-155.

Jun, H., Loganathan, G. V., Kim, J. H., and Park, S. (2008). "Identifying pipes and valves of high importance for efficient operation and maintenance of water distribution systems." Water Resour. Manage., 22(6), 719-736.

Jun, L., and Guoping, Y. (2013). "Iterative methodology of pressuredependent demand based on EPANET for pressure-deficient water distribution analysis." J. Water Resour. Plann. Manage., 10.1061/(ASCE) WR.19435452.0000227, 34-44.

Kadu, M. S., R. Gupta, and P. R. Bhave (2008). "Optimal Design of Water Networks Using a Modified Genetic Algorithm with Reduction in Search Space" J. Water Resour. Plan. Manag., 134(2), 147-160, doi:10.1061/(ASCE)0733-9496(2008)134:2(147).

Kamani, H., Malakootian, M., Hoseini, M., and Jaafari, J. (2012). "Management of Non-Revenue Water in Distribution Network and Conveyor Lines; a Case Study." Health Scope, 1(3), 147-152.

Kanakoudis VK (2004). "A troubleshooting manual for handling operational problems in water pipe networks" Water Supply Res Technol AQUA, 53(2):109-124.

Kanakoudis VK, Tolikas DK (2004). "Assessing the performance level of a water system" Water, Air, \& Soil Pollution. , 4(4):307-318.

Kanakoudis, V. and Tolikas, D., (2001). "The role of leaks and breaks in water networks - Technical and economical solutions" Water Supply: Research \& Technology-AQUA, IWAp, 50 (5), 301-311. 
Kang, D. (2014). "Real-time optimal control of water distribution systems." Procedia Eng., 70, 917-923.

Kao, J. J., and Li, P. H. (2007). "A segment-based optimization model for water pipeline replacement." J. Am. Water Works Assoc., 99(7), 83-95.

Kapelan, Z., and Giustolisi, O. (2006). "Risk assessment of water supply interruptions due to mechanical pipe failures." Combined Int. Conf. ofComputing and Control for the Water Industry and Sustainable Urban Water Management, Taylor \& Francis, London.

Klise, K. a., Bynum, M., Moriarty, D., and Murray, R. (2017). "A software framework for assessing the resilience of drinking water systems to disasters with an example earthquake case study." Environmental Modelling \& Software, Elsevier Ltd, 95, 420-431.

Kovalenko, Y., Gorev, N. B., Kodzhespirova, I. F., Prokhorov, E., and Trapaga, G. (2014). "Convergence of a hydraulic solver with pressuredependent demands." J. Water Resour. Manage., 28(4), 1013-1031.

L Reis and T Pinheiro (2005). "Combined genetic algorithm-linear programming (GA-LP) procedure applied to the operation of the Fortaleza water supply reservoir system (Brazil)" In Conference on Computing and Control for the Water Industry.

Lambert, A. O. (2002). "International Report: Water losses management and techniques." Water Science \& Technology: Water Supply, 2(4), 1-20.

Lansey, K. (2012). "Sustainable, robust, resilient, water distribution systems." Proc., Water Distribution System Analysis 2012, Adelaide, Australia, Engineers Australia, Barton ACT, Australia.

Laucelli, D., and O. Giustolisi (2015). "Vulnerability Assessment of Water Distribution Networks under Seismic Actions" J. Water Resour. Plan. Manag., 141(6), 04014082, doi:10.1061/(ASCE)WR.1943-5452.0000478.

Laucelli, D., M. Romano, D. Savic, and O. Giustolisi (2016). "Detecting anomalies in water distribution networks using EPR modelling paradigm" $J$. Hydroinformatics, 18(3), 409-427, doi:10.2166/hydro.2015.113. 
Li, P. H., and Kao, J. J. (2008). "Segment-based vulnerability analysis system for a water distribution network." Civ. Eng. Environ. Syst., 25(1), 41-58.

Little, R. G. (2002). "Controlling cascading failure: Understanding the vulnerabilities of interconnected infrastructures." Journal of Urban Technology, 9(1), 109-123.

Liu, J., Yu, G., and Savic, D. (2011). "Deficient-network simulation considering pressure-dependent demand." Sustainable Solutions for Water, Sewer, Gas, and Oil Pipelines (ICPTT 2011), ASCE, Reston, VA, 886-900.

Maier, H. R. et al. (2014). "Evolutionary algorithms and other metaheuristics in water resources: Current status, research challenges and future directions" Environ. Model. Softw., 62, 271-299, doi:10.1016/j.envsoft.2014.09.013.

Mansoor, M. A. M., and Vairavamoorthy, K. (2003). "Need for pressure dependent demand in analysing failure of pipe networks." Advances in Water Supply Management, 217-225.

Mansoor, M.A. Mohamed (2008). "Performance Assessment of Water Distribution Systems" , PhD Thesis, Loughborough University, Leicestershire, UK.

Marchi, A., et al. (2013). "The battle of the water networks II (BWN-II)." J. Water Resour. Plann. Manage., 10.1061/(ASCE)WR.1943-5452 .0000378, 04014009.

Mays, L. W. (Org.). (2000). "Water distribution systems handbook.", New York: McGraw-Hill.

Mays, LW. (2000). "Water distribution systems handbook" New York: McGrawHill Professional.

Misiunas, D. (2005). "Failure Monitoring and Asset Condition Assessment in Water Supply Systems," PhD Thesis, Lund University, Lund.

Morley, M. S., and Tricarico, C. (2014). "Pressure driven demand extension for EPANET (EPANETpdd)." Technical Rep. 2008, Univ. of Exeter, Exeter, U.K.

Morosini, F. a. (2016). "Emergency Management of Water Distribution Systems: the Nodal Demand Control." Procedia Engineering, 186, 428-435. 
Nayak, M. A., and M. A. Turnquist (2016), Optimal Recovery from Disruptions in Water Distribution Networks, Comput. Civ. Infrastruct. Eng., 31(8), 566-579, doi:10.1111/mice.12200.

Nicklow, J., et al. (2010). "State of the art for genetic algorithms and beyond in water resources planning and management." J. Water Resour. Plann. Manage., 10.1061/(ASCE)WR.1943-5452.0000053, 412-432.

Odan, F., Ribeiro Reis, L., and Kapelan, Z. (2015). "Real-time multiobjective optimization of operation of water supply systems." J. Water Resour. Plann. Manage., 10.1061/(ASCE)WR.1943-5452.0000515, 04015011.

OFWAT (2013). "Key indicators guidance", https://www.ofwat.gov.uk/wpontent/uploads/2015/11/prs web kpiprintable.pdf, (accessed 17 March 2018).

OFWAT (2018a). "Supply interruptions", https://www.ofwat.gov.uk /nonhouseholds /supply-and-standards/ supply-interruptions/, (accessed 17 March 2018).

OFWAT (2018b). "Water pressure", https://www.ofwat.gov.uk/nonhouseholds /supply-and-standards/water-pressure/, (accessed 17 March 2018).

OFWAT (2018c). "Standards of service", https://www.ofwat.gov.uk/households/ supply-and-standards/standards-of-service/, (accessed 17 March 2018).

O'Rourke, T. D. (2007). "Critical infrastructure, interdependencies, and resilience." Bridge, 37(1), 22-29.

Osman, H., and Bainbridge, K. (2011). "Comparison of statistical deterioration models for water distribution networks." J. Perform. Constr. Facil., 10.1061/(ASCE)CF.1943-5509.0000157, 259-266.

Ostfeld, A. (2001). Reliability analysis of regional water distribution systems, Urban Water, 3(4), 253-260.

Ostfeld, A. (2004). "Reliability analysis of water distribution systems." J. Hydroinf., 6(4), 281-294.

Ostfeld, A., et al. (2008). "The battle of the water sensor networks (BWSN): A design challenge for engineers and algorithms." J. Water Resour. Plann. Manage., 10.1061/(ASCE)0733-9496(2008)134\%3A6 (556), 556-568. 
Ostfeld, A., et al. (2012). "Battle of the water calibration networks." J. Water Resour. Plann. Manage., 10.1061/(ASCE)WR.1943-5452 .0000191, 523532.

Ostfeld, A., Kessler, A., and Goldberg, I. (2004). "A contaminant detection system for early warning in water distribution networks." Eng. Optimiz., 365, 525538.

Ostfeld, A., Kogan, D., and Shamir, U. (2002). "Reliability simulation of water distribution systems-Single and multiquality." Urban Water, 4, 53-61.

Ozger, S. S., and Mays, L. W. (2003). "A semi-pressure-driven approach to reliability assessment of water distribution networks." Proc., 30th Int. Association of Hydraulic Research Congress, Theme B-Urban and Rural Water Systems for Sustainable Development, Aristoteleio Panepistimio Thessalonikis, Thessaloniki, Greece, 345-352.

Pasha, M., and Lansey, K. (2011). "Strategies for real time pump operation for water distribution systems." Proc., Water Distribution Systems Analysis, ASCE, Reston, VA, 679-686.

Penrose, R. (1955). "A Generalized Inverse for Matrices." Proc., Cambridge Phil. Soc., 51, 406-413.

Piller, O., and van Zyl, J. E. (2007). "A unified framework for pressure driven network analysis." Proc., Computer and Control in Water Industry, Taylor \& Francis Group, London, 25-30.

Piller, O., and Van Zyl, J. E. (2009). "Pressure-driven analysis of network sections supplied via high-lying nodes" Taylor \& Francis Group, London.

Poulin, A., A. Mailhot, P. Grondin, L. Delorme, N. Periche, and J.-P. Villeneuve (2008). "Heuristic Approach for Operational Response to Drinking Water Contamination" J. Water Resour. Plan. Manag., 134(5), 457-465, doi:10.1061/(ASCE)0733-9496(2008)134:5(457).

Poulin, A., Mailhot, A., Periche, N., Delorme, L., Villeneuve, and J.-P., (2010). "Planning Unidirectional Flushing Operations as a Response to Drinking Water Distribution System Contamination" Journal of Water Resources Planning and Management, 136(6), 647-657. 
Prasad, T. D., and N.-S. Park. (2004). "Multiobjective Genetic Algorithms for Design of Water Distribution Networks" J. Water Resour. Plan. Manag., 130(1), 73-82, doi:10.1061/(ASCE)0733-9496(2004)130:1(73).

Preis, A., and A. Ostfeld (2008). "Multiobjective contaminant response modeling for water distribution systems security" J. Hydroinformatics, 10(4), 267, doi:10.2166/hydro.2008.061.

Preis, A., Y. Mayorchik, and A. Ostfeld (2007). "Multiobjective Contaminant Detection Response Model" World Environ. Water Resour. Congr. 2007 Restoring Our Nat. Habitat Multiobjective, 1-12.

Puleo, V., Fontanazza, C. M., Notaro, V., and Freni, G. (2014). "Multi sources water supply system optimal control: A case study" Procedia Engineering, 89, 247-254.

Rasekh, A., and K. Brumbelow (2014). "Drinking water distribution systems contamination management to reduce public health impacts and system service interruptions" Environ. Model. Softw., 51, 12-25, doi:10.1016/j.envsoft.2013.09.019.

Rasekh, A., and K. Brumbelow (2015). "A dynamic simulation-optimization model for adaptive management of urban water distribution system contamination threats" Appl. Soft Comput., 32, 59-71, doi:10.1016/j.asoc.2015.03.021.

Rasekh, A., Hassanzadeh, A., Mulchandani, S., Modi, S., and Banks, M. K. (2016). "Smart water networks and cyber security." J. Water Resour. Plann. Manage., 10.1061/(ASCE)WR.1943-5452.0000646, 01816004.

Rasekh, A., K. Brumbelow, and M. K. Lindell (2014), Water as Warning Medium: Food-Grade Dye Injection for Drinking Water Contamination Emergency Response, J. Water Resour. Plan. Manag., 140(1), 12-21, doi:10.1061/(ASCE)WR.1943-5452.0000322.

Reed, P., B. S. Minsker, and D. E. Goldberg (2003). "Simplifying multiobjective optimization: An automated design methodology for the nondominated sorted genetic algorithm-II" Water Resour. Res., 39(7), doi:10.1029/2002WR001483. 
Romano, M., and Z. Kapelan (2014). "Adaptive water demand forecasting for near real-time management of smart water distribution systems" Environ. Model. Softw., 60(0), 265-276, doi:10.1016/j.envsoft.2014.06.016.

Romano, M., Kapelan, Z., and Savic, D. (2014). "Automated detection of pipe bursts and other events." J. Water Resour. Plann. Manage., 10.1061 /(ASCE)WR.1943-5452.0000339, 457-467.

Rossman, L. A. (2000a). "EPANET 2: User's manual" U.S. Environmental Protection Agency, Cincinnati.

Rossman, L. A. (2000b). "EPANET2 and programmer's toolkits" Risk Reduction Engineering Laboratory, U.S. Environmental Protection Agency, Cincinnati.

Salgado-Castro, R. O. (1988). "Computer modelling of water supply distribution networks using the gradient method" Ph.D. thesis, Univ. of Newcastle, Newcastle upon Tyne, U.K.

Savic, D., Vamvakeridou-Lyroudia, L., Kapelan, Z., (2014). "Smart meters, smart water, smart societies: the iwidget project." Procedia Eng. 89, 1105e1112.

Sayyed, M. A. H. A., Gupta, R., and Tanyimboh, T. T. (2015). "Noniterative application of EPANET for pressure dependent modelling of water distribution systems." J. Water Resour. Manage., 29(9), 3227-3242.

Sayyed, M. A., Gupta, R., and Tanyimboh, T. T. (2014). "Modelling pressure deficient water distribution networks in EPANET." Procedia Eng., 89, 626631.

Schraudolph NN, Belew RK (1992). "Dynamic parameter encoding for genetic algorithms" Mach Learn 9(1):9-21.

Seth, A., K. A. Klise, J. D. Siirola, T. Haxton, and C. D. Laird (2016). "Testing Contamination Source Identification Methods for Water Distribution Networks" J. Water Resour. Plan. Manag., 142(4), 04016001, doi:10.1061/(ASCE)WR.1943-5452.0000619.

Shafiee, M.E. and E.M. Zechman (2013). "An agent-based modeling framework for sociotechnical simulation of water distribution contamination events" $J$. Hydroinform. 15 (3) 862-880. 
Shin, S., Lee, S., Judi, D., Parvania, M., Goharian, E., McPherson, T., and Burian, S. (2018). "A Systematic Review of Quantitative Resilience Measures for Water Infrastructure Systems." Water, 10, 164; doi:10.3390/w10020164.

Shirzad, A., Tabesh, M., Farmani, R., and Mohammadi, M. (2012). "Pressuredischarge relations with application in head driven simulation of water distribution networks." J. Water Resour. Plann. Manage., 10.1061/(ASCE)WR.1943-5452.0000305, 660-670.

Shuang, Qing; Liu, Yisheng; Tang, Yongzhong; Liu, Jing; Shuang, Kai. (2017). "System Reliability Evaluation in Water Distribution Networks with the Impact of Valves Experiencing Cascading Failures." Water 9, no. 6: 413.

Siew, C., and Tanyimboh, T. T. (2012). "Pressure-dependent EPANET extension." J. Water Resour. Manage., 26(6), 1477-1498.

Simpson, A. R., Dandy, G. C., and Murphy, L. J. (1994). "Genetic algorithms compared to other techniques for pipe optimization." J. Water Resour. Plann. Manage., 10.1061/(ASCE)0733-9496(1994) 120:4(423), 423-443.

Sivakumar, P., and Prasad, R. K. (2015). "Extended period simulation of pressure-deficient networks using pressure reducing valves." Water Resour. Manage., 29(5), 1713-1730.

Su, Y. L., Mays, L. W., Duan, N., and Lansey, K. E. (1987). "Reliabilitybased optimization model for water distribution systems." J. Hydraul. Eng., 113(12), 1539-1556.

Sunela, Markus (2017). "Real-Time Control Optimization ofWater Distribution System with Storage" PhD Thesis, Tallinn University of Technology, Estonia.

Suribabu, C. R., and Neelakantan, T. (2011). "Balancing reservoir based approach for solution to pressure deficient water distribution networks." Int. J. Civ. Struct. Eng., 2(2), 648-656.

Tabesh, M., Jamasb, M., and Moeini, R. (2011). "Calibration of water distribution hydraulic models: A comparison between pressure dependent and demand driven analyses." Urban Water J., 8(2), 93-102. 
Tanyimboh, T. T., Burd, R., Burrows, R., and Tabesh, M. (1999). "Modelling and reliability analysis of water distribution systems." Water Sci. Technol., 39(4), 249-255.

Tanyimboh, T., and Templeman, A. (2010). "Seamless pressure-deficient water distribution system model." J. Water Manage., 163(8), 389-396.

Thames Water (2015), "Mains design guidelines for self-lay organisations working in new developments", https://www.thameswater.co.uk//media/Site-Content/ Developer-Services/Mains-design-guidelines.pdf , (accessed 17 March 2018).

Tian, Y., G. Y. Fu, H. Chi, and Y. Liu (2007). "Optimal operation of water distribution networks under local pipe failures" J. Cent. South Univ. Technol., 14(3), 436-441, doi:10.1007/s11771-007-0085-1.

Todini, E. (2000). "Looped water distribution networks design using a resilience index based heuristic approach." Urban Water, 2(2), 115-122.

Todini, E. (2006). "Towards realistic extended period simulations (EPS) in looped pipe network." Proc., 8th Annual Int. Symp. on Water Distribution Systems Analysis, ASCE, Reston, VA.

Todini, E., and Pilati, S. (1988). "A gradient algorithm for the analysis of pipe networks" Wiley, London, 1-20.

Turner, J. P., J. Qiao, M. Lawley, J.-P. Richard, and D. M. Abraham (2012). "Mitigating shortage and distribution costs in damaged water networks" Socioecon. Plann. Sci., 46(4), 315-326, doi:10.1016/j.seps.2012.02.001.

US Environmental Protection Agency (2011). "Planning for an Emergency Drinking Water Supply" US Environmental Protection Agency, Office of Research and Development, National Homeland Security Research Center. EPA 600/R-11/054.

US Environmental Protection Agency (2014). "Flood Resilience: a Basic Guide for Water and Wastewater Utilities" Office of Water, EPA 817/B-14/006. US Environmental Protection Agency, Washington DC. 
US Environmental Protection Agency (2014). "Systems Measures of Water Distribution System Resilience" Office of Water, EPA 600/R-14/383. US Environmental Protection Agency, Washington DC.

US Environmental Protection Agency (2015). "Power Resilience Guide for Water and Wastewater Utilities" Office of Water, EPA 600/Re15/004. US Environmental Protection Agency, Washington DC.

US Environmental Protection Agency (2016). "Drought Response and Recovery: a Basic Guide for Water Utilities" Office of Water, EPA 810/B-16/001. US Environmental Protection Agency, Washington DC.

Vairagade, S. A., Sayyed, M. A. H. A., and Gupta, R. (2015). "Node head flow relationships in skeletonized water distribution networks for predicting performance under deficient conditions." World Environmental and Water Resources Congress 2015, ASCE, Reston, VA, 810-819.

Vamvakeridou-Lyroudia, L. S., J. Bicik, M. Morley, D. Savic, and Z. Kapelan (2011). "A Real-Time Intervention Management Model for Reducing Impacts Due to Pipe Isolation in Water Distribution Systems" in Water Distribution Systems Analysis 2010, pp. 209-221, American Society of Civil Engineers, Reston, VA.

Van Zyl, J. E., and Clayton, C. R. I. (2005). "The Effect of Pressure on Leakage in Water Distribution Systems." Computing and Control in the Water Industry (CCWI 2005), Exeter, UK, 131-136.

Van Zyl, J. E., and Clayton, C. R. I. (2007). "The effect of pressure on leakage in water distribution systems." Proceedings of ICE, Water Management, 160(WM2), 109-114.

Wagner, B. J. M., Shamir, U., and Marks, D. H. (1988). "Water distribution reliability: Simulation method." J. Water Resour. Plann. Manage., 10.1061/(ASCE)0733-9496(1988)114:3(276), 276-294.

Walski T, Blakley D, Evans M, Whitman B (2017) "Verifying Pressure Dependent Demand Modeling.", Procedia Engineering, 186:364-371

Walski, T. (2015). "Can Real-Time Modeling Be Useful for Emergency Planning and Response?" World Environmental and Water Resources Congress 2015, American Society of Civil Engineers, Reston, VA, 685-695. 
Walski, T. M. (1993). "Water distribution valve topology for reliability analysis." Reliab. Eng. Syst. Saf., 42(1), 21-27.

Walski, T. M. (2011). "How many isolation valves are needed in a water distribution system?” ,CCWI Conf., Univ. of Exeter, Exeter, 77-82.

Walski, T. M., Chase, D. V., Savić, D. A., Grayman, W., Beckwith, S., and Koelle, E. (2003). "Advanced water distribution modeling and management" Haestead Press, Waterbury, Connecticut.

Wang, Q., M. Guidolin, D. Savic, and Z. Kapelan (2013). “Two-Objective Design of Benchmark Problems of a Water Distribution System via MOEAs: Towards the Best-Known Approximation of the True Pareto Front" J. Water Resour. Plan. Manag., 139(June), 554-564.

Wood, D. J., and Charles, O. A. (1970). "Hydraulic network analysis using linear theory." J. Hydraul. Div., 96(7), 1221-1234.

Wu, Z. Y. (2007). "Discussion of 'Solution for water distribution systems under pressure-deficient conditions' by Wah Khim Ang and Paul W. Jowitt." J. Water Resour. Plann. Manage., 10.1061/(ASCE)07339496(2007)133:6(567), 565-581.

Wu, Z. Y., and Walski, T. (2006). "Pressure dependent hydraulic modelling for water distribution systems under abnormal conditions." IWA World Water Congress and Exhibition, Beijing.

Wu, Z. Y., Wang, R. H., Walski, T. M., Yang, S. Y., Bowdler, D., and Baggett, C. C. (2009). "Extended global-gradient algorithm for pressure-dependent water distribution analysis." J. WaterResour. Plann. Manage., 10.1061/(ASCE)0733-9496(2009)135:1(13), 13-22.

Yoo, D. G., Kang, D., Jun, H., and Kim, J. H. (2014). "Rehabilitation priority determination of water pipes based on hydraulic importance." Water (Switzerland), 6(12), 3864-3887.

Yoo, D. G., Suh, M. Y., Kim, J. H., Jun, H., and Chung, G. (2012). "Subsystembased pressure dependent demand analysis in water distribution systems using effective supply." J. Civil Eng., 16(3), 457-464. 
Zhang, Y. (1996). "Solving Large-Scale Linear Programs by Interior-Point Methods under the MATLAB", Optim. Methods Softw., 10 (1998), pp. 1-31.

Zheng, F., A. R. Simpson, A. C. Zecchin, and J. W. Deuerlein (2013). "A graph decomposition-based approach for water distribution network optimization" Water Resour. Res., 49(4), 2093-2109, doi:10.1002/wrcr.20175.

Zheng, F., A. R. Simpson, and A. C. Zecchin (2011). "A combined NLP-differential evolution algorithm approach for the optimization of looped water distribution systems" Water Resour. Res., 47(8), doi:10.1029/2011WR010394.

Zhuang, B., Lansey, K., and Kang, D. (2013). "Resilience/availability analysis of municipal water distribution system incorporating adaptive pump operation." J. Hydraul. Eng., 10.1061/(ASCE)HY.1943-7900 .0000676, 527-537. 


\section{Appendix A Segment Analysis Detail}

Table A1. Segment analysis for D-Town

\begin{tabular}{|c|c|c|c|c|c|}
\hline & \begin{tabular}{|l|}
$\begin{array}{l}\text { Regular isolated } \\
\text { links }\end{array}$ \\
\end{tabular} & $\begin{array}{l}\text { Unintended isolated } \\
\text { links }\end{array}$ & $\begin{array}{l}\text { Regular isolated } \\
\text { nodes }\end{array}$ & $\begin{array}{l}\text { Unintended isolated } \\
\text { nodes }\end{array}$ & \begin{tabular}{|l}
$\begin{array}{l}\text { List of isolation } \\
\text { valves }\end{array}$ \\
\end{tabular} \\
\hline S1 & V47 & P304,P290,P288,P286 & J169,J129 & J224,J222,J182,J152 & P1041,P286,P288 \\
\hline S2 & 12,13 & & N10,N9 & & $8,12,14$ \\
\hline S3 & 10,11 & & N3 & & $9,10,11$ \\
\hline S4 & 9,14 & & N4 & & $7,9,14$ \\
\hline S5 & 7 & & N8 & & 6,7 \\
\hline S6 & $4,5,8$ & & N6,N5 & & 4,8 \\
\hline S7 & $2,3,6, \mathrm{~N} 15$ & & $\mathrm{~N} 11, \mathrm{~N} 7, \mathrm{~N} 1, \mathrm{~J} 145$ & & P94,P95,4,6,10 \\
\hline S8 & 1 & & $\mathrm{~N} 2$ & & $1,11,12$ \\
\hline S9 & P998 & & $\mathrm{J} 204$ & & P1000,P159,P998 \\
\hline S10 & P996 & & J332 & & P397,P996 \\
\hline S11 & P995,P997 & & J201,J308 & & P995,P996,P998 \\
\hline S12 & P991,P992 & & J202 & & P991,P992 \\
\hline S13 & P981 & P978,P976,P249,P161 & J361 & J360,J359,J358,J355 & P161,P981 \\
\hline $\mathrm{S} 14$ & P968,P969,P970,P994 & & $\begin{array}{l}\text { J199,J200,J198, } \\
\text { J1154 }\end{array}$ & & $\begin{array}{l}\text { P967,P990,P993, } \\
\text { P994 }\end{array}$ \\
\hline S15 & P964,P965,P966,P971 & & J205,J367 & & $\begin{array}{l}\text { P963,P964,P966, } \\
\text { P971 }\end{array}$ \\
\hline S16 & P949,P953,v1 & $\begin{array}{l}\text { P946,P944,P942,P941, } \\
\text { P940,P939 }\end{array}$ & J88,J35 & $\mathrm{J} 31, \mathrm{~J} 30, \mathrm{~J} 27, \mathrm{~J} 26, \mathrm{~J} 25$ & P243,P949,P953 \\
\hline S17 & P943 & & $\mathrm{J} 81$ & & P943 \\
\hline
\end{tabular}




\begin{tabular}{|c|c|c|c|c|c|}
\hline S18 & $\begin{array}{l}\text { P939,P940,P941, } \\
\text { P942,P944,P946 }\end{array}$ & & \begin{tabular}{|l|}
$\mathrm{J} 31, \mathrm{~J} 30, \mathrm{~J} 27$ \\
$\mathrm{~J} 26, \mathrm{~J} 25$ \\
\end{tabular} & & P949 \\
\hline S19 & P938 & & $\mathrm{J} 257, \mathrm{~J} 251$ & & P163,P937,P951 \\
\hline S20 & P934,P935,P937 & & J23,J22,J385 & & P1016,P933,P937 \\
\hline S21 & P914,P929,P930 & $\begin{array}{l}\text { P932,P931,P810,P780, } \\
\text { P754,P697,P671,P670, } \\
\text { P610,P510 }\end{array}$ & $\mathrm{J} 21, \mathrm{~J} 401, \mathrm{~J} 399$ & $\begin{array}{l}\text { J20,J19,J18,J17,J16, } \\
\text { J15,J406,J394 }\end{array}$ & P280,P914,P932 \\
\hline S22 & P86 & & J135,J134 & & P165,P385,P386 \\
\hline S23 & P855,P858 & & J92,J76 & & P858 \\
\hline S24 & P821,P822 & & J267,J354 & & P822 \\
\hline S25 & P810,P931 & & J20,J406,J394 & & P780,P914 \\
\hline S26 & P807 & & J350 & & P807 \\
\hline S27 & P805,P806 & $\begin{array}{l}\text { P889,P804,P801, } \\
\text { P800,P241 }\end{array}$ & J266,J265 & $\begin{array}{l}\text { J347,J345,J344, } \\
\text { J1170,J1169 }\end{array}$ & P804,P806 \\
\hline $\mathrm{S} 28$ & P804,P889 & P801,P800,P241 & J1170,J1169 & J347,J345,J344 & P800,P801,P804 \\
\hline S29 & P801 & & J347 & & P801 \\
\hline S30 & P794,P795,P798 & $\begin{array}{l}\text { P889,P807,P806,P805, } \\
\text { P804,P801,P800,P241 }\end{array}$ & J260,J255,J239 & $\begin{array}{l}\text { J266,J265,J350,J347, } \\
\text { J345,J344,J1170, } \\
\text { J1169 }\end{array}$ & P794,P806,P807 \\
\hline S31 & P789,P961,P963 & & J366 & & P789,P961,P963 \\
\hline S32 & $\begin{array}{l}\text { P786,P787,P788, } \\
\text { P791,P797 }\end{array}$ & & T3,J258,J227,J216 & & P786,P791 \\
\hline S33 & P785 & & J215 & & P785 \\
\hline S34 & P783,P784 & & J213,J197,J190 & & P779,P785,P786 \\
\hline S35 & P777,P779 & & J185 & & P777,P779 \\
\hline S36 & P775,P776 & & $\mathrm{J} 184, \mathrm{~J} 178$ & & P775 \\
\hline S37 & P771,P772 & & J176,J170,J168 & & P769,P775,P777 \\
\hline S38 & P767,P768,P769 & & J158,J157,J94 & & P763,P769 \\
\hline S39 & $\begin{array}{l}\text { P755,P756,P763, } \\
\text { P94,P95 }\end{array}$ & & $\mathrm{J} 141, \mathrm{~J} 140, \mathrm{~J} 123$ & & P763,P94,P95 \\
\hline
\end{tabular}




\begin{tabular}{|c|c|c|c|c|c|}
\hline S40 & P752 & & T7 & & P752 \\
\hline S41 & P724 & & J268 & & P724 \\
\hline S42 & P68,P924 & P71,P119 & J195 & J191,J189,J188 & P68,P924 \\
\hline S43 & $\begin{array}{l}\text { P610,P670,P671, } \\
\text { P780,P932 }\end{array}$ & P754,P697,P510 & J18,J17 & J19,J16,J15 & $\begin{array}{l}\text { P610,P671, } \\
\text { P780,P932 }\end{array}$ \\
\hline S44 & P597 & & J284,J281 & & P1022,P529 \\
\hline S45 & $\begin{array}{l}\text { P596,P846,P847, } \\
\text { P852 }\end{array}$ & & $\mathrm{J} 118, \mathrm{~J} 58$ & & P596,P847,P852 \\
\hline S46 & P55,P761,P808 & $\begin{array}{l}\text { P781,P766,P309, } \\
\text { P223,P177,P11 }\end{array}$ & J83 & $\begin{array}{l}\mathrm{J} 235, \mathrm{~J} 230, \mathrm{~J} 305, \\
\mathrm{~J} 303, \mathrm{~J} 319, \mathrm{~J} 318\end{array}$ & $\begin{array}{l}\text { P55,P761, } \\
\text { P781,P808 }\end{array}$ \\
\hline S47 & P510,P697,P754 & & J19,J16,J15 & & P610,P671 \\
\hline S48 & P5,P8,P9,P927 & $\begin{array}{l}\text { P90,P89,P70,P69, } \\
\text { P259,P258,P132, } \\
\text { P130,P129,P128,P } \\
\text { 127,P126,P124 }\end{array}$ & $\begin{array}{l}\mathrm{J} 1223, \mathrm{~J} 218 \\
\mathrm{~J} 212, \mathrm{~J} 210\end{array}$ & $\begin{array}{l}\text { J40,J379, J377,J376, } \\
\text { J375,J374,J373, } \\
\text { J372,J226,J225,J316 }\end{array}$ & P21,P69,P7,P9 \\
\hline S49 & P49,P861 & & $\mathrm{J} 74, \mathrm{~J} 72$ & & P861 \\
\hline S50 & P46,P866,P871 & & $\mathrm{J} 61, \mathrm{~J} 70, \mathrm{~J} 69$ & & P866 \\
\hline S51 & $\begin{array}{l}\mathrm{P} 446, \mathrm{P} 450, \mathrm{P} 465, \mathrm{P} 467 \\
\text {,V2 }\end{array}$ & & $\begin{array}{l}\mathrm{J} 14, \mathrm{~J} 422, \mathrm{~J} 421 \\
\mathrm{~J} 418\end{array}$ & & P445,P467 \\
\hline S52 & P443,P445,P892,P96 & & $\begin{array}{l}\mathrm{J} 13, \mathrm{~J} 12, \mathrm{~J} 417, \\
\mathrm{~J} 414\end{array}$ & & P307,P445,P484 \\
\hline S53 & $\mathrm{P} 44, \mathrm{P} 633$ & $\begin{array}{l}\text { P871,P866,P861, } \\
\text { P859,P858,P855, } \\
\text { P51,P49,P48,P46,P42 }\end{array}$ & J67 & $\begin{array}{l}\mathrm{J} 92, \mathrm{~J} 61, \mathrm{~J} 77, \mathrm{~J} 76, \\
\mathrm{~J} 74, \mathrm{~J} 73, \mathrm{~J} 72, \mathrm{~J} 71 \\
\mathrm{~J} 70, \mathrm{~J} 69, \mathrm{~J} 68, \mathrm{~J} 54\end{array}$ & $\begin{array}{l}\text { P44,P633, } \\
\text { P852,P853 }\end{array}$ \\
\hline S54 & P424,P53 & & J384 & & P424,P53 \\
\hline S55 & P42,P48,P51,P859 & $\begin{array}{l}\text { P871,P866,P861,P858, } \\
\text { P855,P49,P46 }\end{array}$ & $\mathrm{J} 77, \mathrm{~J} 73, \mathrm{~J} 71, \mathrm{~J} 54$ & $\begin{array}{l}\text { J92,J61,J76,J74, } \\
\text { J72,J70,J69,J68 }\end{array}$ & P51,P633,P861 \\
\hline S56 & $\begin{array}{l}\text { P399,P402,P410,PU10, } \\
\text { PU11 }\end{array}$ & & $\begin{array}{l}\mathrm{J} 323, \mathrm{~J} 317 \\
\mathrm{~J} 309, \mathrm{~J} 307\end{array}$ & & P399,P424 \\
\hline S57 & P384 & & J575 & & P1025,P384 \\
\hline S58 & P380,P381,PU6,PU7 & & J291,J290, & & P237,P379 \\
\hline
\end{tabular}




\begin{tabular}{|c|c|c|c|c|c|}
\hline & & & J289,J415 & & \\
\hline S59 & P38,P609,P842 & & J56,J55 & & $\begin{array}{l}\text { P596,P609, } \\
\text { P842,P847 }\end{array}$ \\
\hline $\mathrm{S} 60$ & $\begin{array}{l}\text { P375,P376,P378,P796 } \\
\text {,PU4,PU5 } \\
\end{array}$ & & $\begin{array}{l}\mathrm{J} 300, \mathrm{~J} 299, \mathrm{~J} 292, \\
\mathrm{~J} 288, \mathrm{~J} 256, \mathrm{~J} 238\end{array}$ & & P374,P791,P794 \\
\hline S61 & P37,P986 & & $\mathrm{J} 52, \mathrm{~J} 381, \mathrm{~J} 348$ & & P984,P987 \\
\hline S62 & P347,P348,P349,P350 & $\begin{array}{l}\text { P346,P339,P338, } \\
\text { P330,P329 }\end{array}$ & $\begin{array}{l}\text { T4,J240,J509, } \\
\text { J503 }\end{array}$ & $\begin{array}{l}\text { J504,J502,J501, } \\
\text { J499,J500 }\end{array}$ & P329,P349 \\
\hline S63 & P344,P383 & P501,P267 & $\mathrm{J} 571$ & $\mathrm{~J} 147, \mathrm{~J} 572$ & P344,P383,P501 \\
\hline S64 & $\begin{array}{l}\text { P336,P340,P341,P343, } \\
\text { P385,P386 }\end{array}$ & & $\begin{array}{l}\mathrm{J} 498, \mathrm{~J} 496, \\
\mathrm{~J} 495, \mathrm{~J} 576\end{array}$ & & $\begin{array}{l}\text { P340,P344, } \\
\text { P385,P386 }\end{array}$ \\
\hline S65 & $\begin{array}{l}\text { P329,P330,P338,P339, } \\
\text { P346 }\end{array}$ & & $\begin{array}{l}\text { J504,J502,J501, } \\
\text { J499,J500 }\end{array}$ & & P329 \\
\hline S66 & P32 & & $\mathrm{J} 2$ & & P103,P32 \\
\hline S67 & P298,P301 & & $\mathrm{J} 11, \mathrm{~J} 434$ & & P298,P302 \\
\hline S68 & P291,P293,P308 & & $\mathrm{J} 427, \mathrm{~J} 426, \mathrm{~J} 425$ & & P292,P308 \\
\hline S69 & P288,P290,P304 & & J224,J222,J152 & & P288 \\
\hline S70 & P286 & & J182 & & P286 \\
\hline S71 & P284,P285,P502 & & J153,J151,J574 & & P502 \\
\hline S72 & P282,P294,P299,P303 & & $\begin{array}{l}\mathrm{J} 10, \mathrm{~J} 439, \mathrm{~J} 436, \\
\mathrm{~J} 429, \mathrm{~J} 428\end{array}$ & & P295 \\
\hline S73 & P280,P305 & $\begin{array}{l}\text { P932,P931,P930,P929, } \\
\text { P914,P810,P780,P754, } \\
\text { P697,P671,P670,P610, } \\
\text { P510 }\end{array}$ & J407 & $\begin{array}{l}\text { J21,J20,J19,J18, } \\
\text { J17,J16,J15,J406, } \\
\text { J401,J399,J394 }\end{array}$ & P280,P305 \\
\hline S74 & P27,P28,P29 & P30,P104,P101 & $\begin{array}{l}\mathrm{J} 1, \mathrm{~J} 165, \mathrm{~J} 164, \\
\mathrm{~J} 161\end{array}$ & J167,J166,J162 & P26,P30 \\
\hline S75 & P268,P270,P275 & & J150,J149,J148 & & P268 \\
\hline S76 & P267,P501 & & $\mathrm{J} 147, \mathrm{~J} 572$ & & P501 \\
\hline S77 & $\begin{array}{l}\text { P255,P817,P819,P972, } \\
\text { P973,P974,P975,P989, }\end{array}$ & & $\begin{array}{l}\text { J370,J369,J365, } \\
\text { J444,J1158,J1157, }\end{array}$ & & $\begin{array}{l}\text { P961,P964,P971, } \\
\text { P973,P990, }\end{array}$ \\
\hline
\end{tabular}




\begin{tabular}{|c|c|c|c|c|c|}
\hline & P990 & & J1155,J1153 & & P994,P995 \\
\hline S78 & P252,P256,P933 & & $\mathrm{J} 371, \mathrm{~J} 364, \mathrm{~J} 363$ & & P308,P933 \\
\hline S79 & P249,P976 & & J360,J359 & & P976 \\
\hline $\mathrm{S} 80$ & $\begin{array}{l}\text { P242,P243,P947,P948, } \\
\text { P954,P955,P956,P957, } \\
\text { P958,P959 }\end{array}$ & P943 & $\begin{array}{l}\text { J38,J36,J34,J33, } \\
\text { J32,J29,J28 }\end{array}$ & J81 & $\begin{array}{l}\text { P243,P943,P947, } \\
\text { P955,P958,1 }\end{array}$ \\
\hline S81 & P241,P800 & & J345,J344 & & P800 \\
\hline S82 & P238,P292 & & J416 & & P238,P292 \\
\hline S83 & $\mathrm{P} 237$ & & J441 & & P237,P238,P305 \\
\hline S84 & P231,P841 & & $\mathrm{J} 331, \mathrm{~J} 330$ & & P230,P841 \\
\hline S85 & P228,P230,P813,P840 & P811,P809,P225 & J328,J327,J320 & J324,J322,J321 & $\begin{array}{l}\text { P230,P811, } \\
\text { P815,P840 }\end{array}$ \\
\hline S86 & P225,P809,P811 & & J324,J322,J321 & & P811 \\
\hline S87 & P223,P781 & P766,P309,P177,P11 & J319,J318 & $\begin{array}{l}\mathrm{J} 235, \mathrm{~J} 230, \mathrm{~J} 305 \\
\mathrm{~J} 303\end{array}$ & P11,P766,P781 \\
\hline S88 & P220,P993 & & J315,J314 & & P992,P993 \\
\hline S89 & P219,P759,P760 & & $\mathrm{J} 298, \mathrm{~J} 313, \mathrm{~J} 312$ & & P759,P761 \\
\hline S90 & P218 & & $\mathrm{J} 311, \mathrm{~J} 310$ & & P808,P840 \\
\hline S91 & $\begin{array}{l}\text { P215,P287,P374, } \\
\text { P379,P468 }\end{array}$ & & T2,J287,J420,J419 & & P374,P379,P467 \\
\hline S92 & P211,P725 & & J296,J295 & & P725 \\
\hline S93 & P201,P272,P482 & & J9,J8,J7 & & P174,P195,P482 \\
\hline S94 & P20,P21 & & J214 & & P20,P21,P991 \\
\hline S95 & P184,P251,P815,P915 & & J1208,J362,J329 & & $\begin{array}{l}\text { P16,P184,P53, } \\
\text { P815 }\end{array}$ \\
\hline S96 & P18,P19,P297,P484 & & $\mathrm{J} 438, \mathrm{~J} 411$ & & $\begin{array}{l}\text { P18,P19,P298, } \\
\text { P484 }\end{array}$ \\
\hline S97 & P177,P766 & & J305,J303 & & P766 \\
\hline S98 & P174,P962 & & $\mathrm{J} 6, \mathrm{~J} 1058$ & & $\mathrm{P} 174$ \\
\hline S99 & P166,P982,P983,P984 & & $\mathrm{J} 382, \mathrm{~J} 352, \mathrm{~J} 349$ & & P144,P184,P982, \\
\hline
\end{tabular}




\begin{tabular}{|c|c|c|c|c|c|}
\hline & & & & & P984,P988 \\
\hline$S 100$ & P163,P500 & & J90,J252 & & P163 \\
\hline S101 & $\begin{array}{l}\text { P162,P245,P397,P398, } \\
\text { P403,P409,P57, } \\
\text { PU8,PU9 }\end{array}$ & & $\begin{array}{l}\text { J306,J304,J302, } \\
\text { J301, J87,J85,J84 }\end{array}$ & & $\begin{array}{l}\text { P162,P397, } \\
\text { P399,P58 }\end{array}$ \\
\hline S102 & P161,P978 & P976,P249 & J358,J355 & J360,J359 & P161,P976 \\
\hline S103 & P16,P65 & P822,P821 & J353,J351 & J267,J354 & $\begin{array}{l}\text { P16,P822,P981, } \\
\text { P982 }\end{array}$ \\
\hline $\mathrm{S} 104$ & P158,P160,P823,P826 & & $\mathrm{J} 246, \mathrm{~J} 237, \mathrm{~J} 236$ & & P160,P826 \\
\hline S105 & P157,P827,P830 & & J247,J244 & & \begin{tabular}{|l|} 
P157,P826,P830 \\
\end{tabular} \\
\hline S106 & $\begin{array}{l}\text { P156,P246,P248, } \\
\text { P264,P266, } \\
\text { P492,P831 }\end{array}$ & & $\begin{array}{l}\text { J250,J249,J243, } \\
\text { J242,J241 }\end{array}$ & & $\begin{array}{l}\text { P162,P248, } \\
\text { P492,P831 }\end{array}$ \\
\hline S107 & $\begin{array}{l}\text { P155,P40,P844, } \\
\text { P850,P851 }\end{array}$ & & $\mathrm{J} 62, \mathrm{~J} 60, \mathrm{~J} 59, \mathrm{~J} 57$ & & P155,P609,P842 \\
\hline $\mathrm{S} 108$ & $\mathrm{P} 154$ & & J245,J66 & & P157,P248,P44 \\
\hline S109 & P150,P35 & & $\mathrm{J} 82, \mathrm{~J} 50$ & & P35,P55 \\
\hline $\mathrm{S} 110$ & P15 & & T1 & & P15 \\
\hline S111 & P147,P987,P988 & & $\mathrm{J} 232, \mathrm{~J} 231$ & & P987,P988 \\
\hline $\mathrm{S} 112$ & $\mathrm{P} 144$ & & T6 & & $\mathrm{P} 144$ \\
\hline $\mathrm{S} 113$ & P142,P951 & & J93,J37 & & P951,P953 \\
\hline S114 & P141,P52,P753 & P725,P724,P211 & $\begin{array}{l}\text { J297,J78,J51, } \\
\text { J221 }\end{array}$ & J268,J296,J295 & $\begin{array}{l}\text { P35,P724,P725, } \\
\text { P752,P759,P841 } \\
\end{array}$ \\
\hline S115 & P140,P372,P58 & & J86,J220,J219 & & P155,P492,P58 \\
\hline S116 & P139,P7 & & J211 & & P139,P7 \\
\hline S117 & P134,P136,P195 & & J5,J4 & & P136,P195 \\
\hline S118 & P131,P967 & & J207,J206 & & P966,P967 \\
\hline S119 & P13,P14 & & J39,J24 & & $\mathrm{P} 13, \mathrm{P} 15$ \\
\hline S120 & $\begin{array}{l}\text { P126,P127,P128, } \\
\text { P129,P259, }\end{array}$ & $\begin{array}{l}\text { P90,P89,P258, } \\
\text { P132,P130,P124 }\end{array}$ & $\begin{array}{l}\mathrm{J} 40, \mathrm{~J} 377, \mathrm{~J} 375, \\
\mathrm{~J} 374, \mathrm{~J} 225, \mathrm{~J} 316\end{array}$ & $\begin{array}{l}\text { J379, J376,J373, } \\
\text { J372,J226 }\end{array}$ & $\begin{array}{l}\text { P124,P128, } \\
\text { P69,P89 }\end{array}$ \\
\hline
\end{tabular}




\begin{tabular}{|c|c|c|c|c|c|}
\hline & P69,P70 & & & & \\
\hline S121 & P125,P26 & $\begin{array}{l}\text { P30,P29,P28,P27, } \\
\text { P104,P101 }\end{array}$ & J163 & $\begin{array}{l}\mathrm{J} 1 \text {,J167,J166,J165, } \\
\mathrm{J} 164, \mathrm{~J} 162, \mathrm{~J} 161\end{array}$ & P125,P26 \\
\hline S122 & $\begin{array}{l}\text { P124,P130,P132, } \\
\text { P258,P89,P90 }\end{array}$ & & $\begin{array}{l}\text { J379,J376,J373, } \\
\text { J372,J226 }\end{array}$ & & P124,P89 \\
\hline S123 & P123,P880 & & J3,J1161,J1160 & & P120,P482 \\
\hline S124 & P122,P2,P3 & & J181,J173 & & P113,P122,P2 \\
\hline S125 & $\mathrm{P} 121, \mathrm{P} 22$ & & J193,J192 & & P22,P23 \\
\hline S126 & P12,P159,P235 & P234,P233,P10 & J208,J337,J336 & $\mathrm{J} 335, \mathrm{~J} 334, \mathrm{~J} 333$ & $\begin{array}{l}\text { P10,P13,P139, } \\
\text { P159 }\end{array}$ \\
\hline S127 & P119,P71 & & J191,J189,J188 & & P68 \\
\hline S128 & $\begin{array}{l}\text { P118,P120,P296, } \\
\text { P302,P307 }\end{array}$ & & $\mathrm{J} 433, \mathrm{~J} 432$ & & $\begin{array}{l}\text { P118,P120, } \\
\text { P302,P307 }\end{array}$ \\
\hline S129 & P115,P117,P295 & $\begin{array}{l}\text { P303,P299, } \\
\text { P294,P282 }\end{array}$ & $\mathrm{J} 435, \mathrm{~J} 431$ & $\begin{array}{l}\mathrm{J} 10, \mathrm{~J} 439, \mathrm{~J} 436, \\
\mathrm{~J} 429, \mathrm{~J} 428\end{array}$ & $\begin{array}{l}\text { P115,P118, } \\
\text { P136,P295 }\end{array}$ \\
\hline $\mathrm{S} 130$ & P112,P757,P758 & P91,P111 & J1024,J142 & J976,J144,J143 & P112,P758 \\
\hline $\mathrm{S} 131$ & P111,P91 & & J976,J144,J143 & & $\mathrm{P} 112$ \\
\hline S132 & P110,P23,P24,P25 & $\begin{array}{l}\text { P30,P29,P28, } \\
\text { P27,P26,P125, } \\
\text { P104,P101 }\end{array}$ & J196,J194,J177 & $\begin{array}{l}\mathrm{J} 1, \mathrm{~J} 167, \mathrm{~J} 166, \\
\mathrm{~J} 165, \mathrm{~J} 164, \\
\mathrm{~J} 163, \mathrm{~J} 162, \mathrm{~J} 161\end{array}$ & $\begin{array}{l}\text { P110,P122, } \\
\text { P125,P23 }\end{array}$ \\
\hline S133 & P11,P309 & & $\mathrm{J} 235, \mathrm{~J} 230$ & & P11 \\
\hline $\mathrm{S} 134$ & $\begin{array}{l}\text { P107,P113,P116,P31, } \\
\text { P6,P84,P87,P92 } \\
\end{array}$ & & $\begin{array}{l}\mathrm{J} 137, \mathrm{~J} 187, \mathrm{~J} 186, \\
\mathrm{~J} 183, \mathrm{~J} 180, \mathrm{~J} 171\end{array}$ & & P107,P113,P32 \\
\hline S135 & P106,P63,P64,P67 & & $\begin{array}{l}\mathrm{J} 102, \mathrm{~J} 101, \mathrm{~J} 97, \\
\mathrm{~J} 96, \mathrm{~J} 95\end{array}$ & & P108,P72 \\
\hline S136 & P1045,P43,P853 & & J65,J64,J53 & & \begin{tabular}{|l|} 
P1044,P853 \\
\end{tabular} \\
\hline S137 & P1044 & & T5 & & P1044 \\
\hline S138 & P1042,P977 & P502,P285,P284 & J91,J89 & J153,J151,J574 & P1042,P502 \\
\hline S139 & $\mathrm{P} 1039, \mathrm{P} 1040, \mathrm{P} 1041$ & $\begin{array}{l}\text { V47,P977,P502,P304, } \\
\text { P290,P288,P286,P285, } \\
\text { P284,P1042 }\end{array}$ & J136,J573,J128 & $\begin{array}{l}\mathrm{J} 224, \mathrm{~J} 222, \mathrm{~J} 182, \\
\mathrm{~J} 169, \mathrm{~J} 153, \mathrm{~J} 152, \mathrm{~J} 151, \\
\mathrm{~J} 574, \mathrm{~J} 91, \mathrm{~J} 89, \mathrm{~J} 129\end{array}$ & $\begin{array}{l}\text { P1035,P1036, } \\
\text { P1041,P1042 }\end{array}$ \\
\hline
\end{tabular}




\begin{tabular}{|c|c|c|c|c|c|}
\hline S140 & P1034,P1035,V45 & P275,P270,P268 & J254,J253,J130 & J150,J149,J148 & P1035,P268 \\
\hline S141 & $\begin{array}{l}\text { P103,P108,P33, } \\
\text { P34,P72,P99 }\end{array}$ & $\begin{array}{l}\text { P924,P71,P68,P67,P64, } \\
\text { P63,P119,P106 }\end{array}$ & $\begin{array}{l}\text { J109,J179, } \\
\text { J1219,J110 }\end{array}$ & $\begin{array}{l}\mathrm{J} 102, \mathrm{~J} 101, \mathrm{~J} 195, \\
\mathrm{~J} 191, \mathrm{~J} 189, \mathrm{~J} 188, \\
\mathrm{~J} 97, \mathrm{~J} 96, \mathrm{~J} 95\end{array}$ & $\begin{array}{l}\text { P102,P103,P108, } \\
\text { P110,P72,P924 }\end{array}$ \\
\hline $\mathrm{S} 142$ & $\begin{array}{l}\mathrm{P} 1027, \mathrm{P} 1028, \mathrm{P} 1029, \\
\mathrm{P} 1031, \mathrm{P} 1032, \mathrm{P} 1033, \\
\mathrm{P} 1036, \mathrm{P} 331, \mathrm{P} 39, \mathrm{P} 54\end{array}$ & $\begin{array}{l}\text { V47,V45,P977,P502, } \\
\text { P304,P290,P288,P286, } \\
\text { P285,P284,P275,P270, } \\
\text { P268,P1042,P1041,P1040, } \\
\text { P1039,P1035,P1034 }\end{array}$ & $\begin{array}{l}\text { J492,J491,J490, } \\
\text { J489,J488,J487, } \\
\text { J486,J131 }\end{array}$ & $\begin{array}{l}\mathrm{J} 224, \mathrm{~J} 222, \mathrm{~J} 182, \\
\mathrm{~J} 169, \mathrm{~J} 153, \mathrm{~J} 152, \mathrm{~J} 151, \\
\mathrm{~J} 150, \mathrm{~J} 149, \mathrm{~J} 148, \mathrm{~J} 136, \\
\mathrm{~J} 574, \mathrm{~J} 573, \mathrm{~J} 91, \mathrm{~J} 89, \mathrm{~J} 254, \\
\mathrm{~J} 253, \mathrm{~J} 130, \mathrm{~J} 129, \mathrm{~J} 128\end{array}$ & $\begin{array}{l}\text { P1030,P1031, } \\
\text { P1036,P383, } \\
\text { P384,P39 }\end{array}$ \\
\hline $\mathrm{S} 143$ & $\begin{array}{l}\text { P1025,P1026, } \\
\text { P1030,P85 }\end{array}$ & & J493,J133,J132 & & P1025,P1030 \\
\hline S144 & $\begin{array}{l}\text { P1024,P337, } \\
\text { P524,P527 }\end{array}$ & P597,P529,P1023,P1022 & $\begin{array}{l}\text { J497,J494,J580, } \\
\text { J511 }\end{array}$ & $\begin{array}{l}\text { J284,J283,J281, } \\
\text { J278 }\end{array}$ & $\begin{array}{l}\text { P1024,P1031, } \\
\text { P340,P349 }\end{array}$ \\
\hline S145 & P1022,P1023,P529 & P597 & $\mathrm{J} 283, \mathrm{~J} 278$ & $\mathrm{~J} 284, \mathrm{~J} 281$ & $\begin{array}{l}\text { P1022,P1024, } \\
\text { P529 }\end{array}$ \\
\hline S146 & P102,P17 & & $\mathrm{J} 408, \mathrm{~J} 1025$ & & \begin{tabular}{|l|}
$\mathrm{P} 102, \mathrm{P} 115$, \\
$\mathrm{P} 18, \mathrm{P} 758$ \\
\end{tabular} \\
\hline S147 & P1016,P148,P165 & & $\mathrm{J} 234, \mathrm{~J} 233$ & & P1016,P165 \\
\hline S148 & P101,P104,P30 & & J167,J166,J162 & & P30 \\
\hline S149 & P1000,P138,P999 & & J203,J341,J217 & & P1000,P9 \\
\hline $\mathrm{S} 150$ & $\begin{array}{l}\text { P100,P310,P316, } \\
\text { P319,P320,P322, } \\
\text { P323,P83,P97, } \\
\text { P98,PU1,PU2,PU3 }\end{array}$ & & $\begin{array}{l}\mathrm{R} 1, \mathrm{~J} 285, \mathrm{~J} 280, \\
\mathrm{~J} 276, \mathrm{~J} 274, \mathrm{~J} 273, \\
\mathrm{~J} 269, \mathrm{~J} 160, \mathrm{~J} 159, \\
\mathrm{~J} 156, \mathrm{~J} 155, \mathrm{~J} 154\end{array}$ & & P22 \\
\hline S151 & P10,P233,P234 & & J335,J334,J333 & & $\mathrm{P} 10$ \\
\hline S152 & P1,P109 & & J175,J174,J172 & & P107,P2 \\
\hline
\end{tabular}


